\title{
Temporal, Spatial and Pulse Instabilities of the Gulf Stream
}

by

\author{
Bingjian Ni \\ B.S., Peking University (1983) \\ M.S., Peking University (1986) \\ M.S.,The College of William and Mary (1990) \\ Submitted in partial fulfillment of the \\ requirements for the degree of \\ DOCTOR OF PHILOSOPHY \\ at the

\section{MASSACHUSETTS INSTITUTE OF TECHNOLOGY}

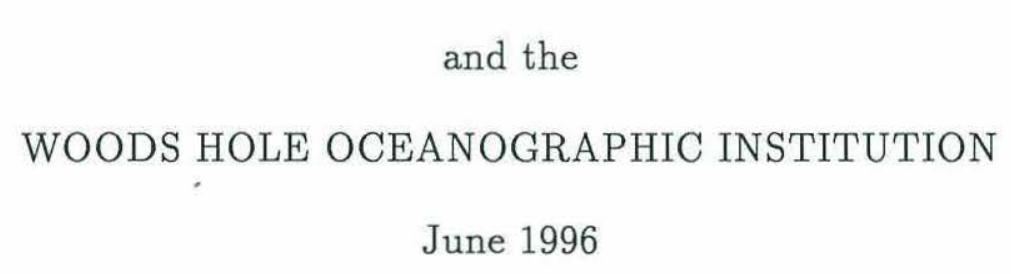 \\ (C) Bingjian Ni 1996
}

The author hereby grants to MIT and to WHOI permission to reproduce

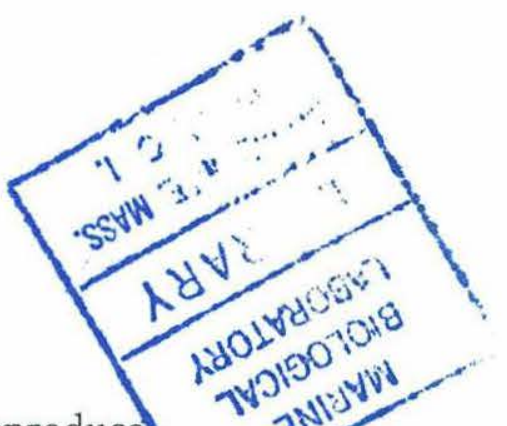
and to distribute copies of this thesis document in whole or in part.

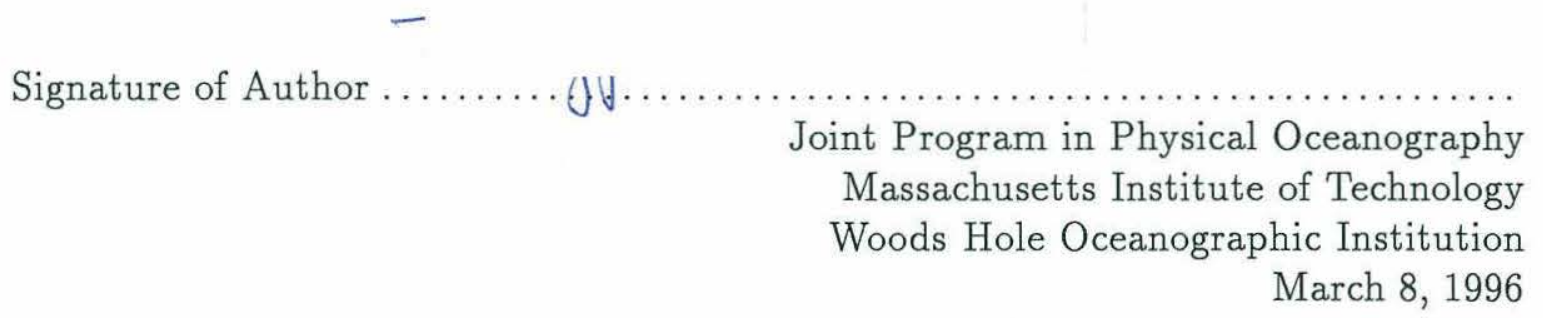

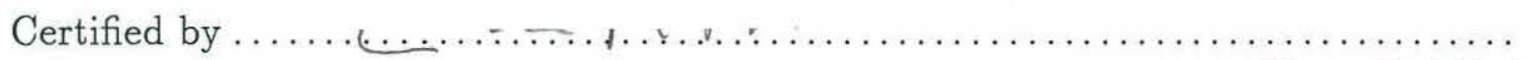

Glenn R. Flierl

Professor

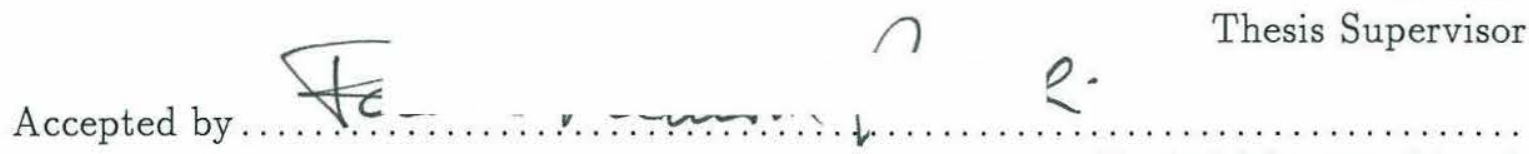

Paola Malanotte-Rizzoli

Chairman, Joint Committee for Physical Oceanography

Massachusetts Institute of Technology

Woods Hole Oceanographic Institution 


\title{
TEMPORAL, SPATIAL AND PULSE INSTABILITIES OF THE GULF STREAM \\ by \\ Bingjian $\mathrm{Ni}$
}

Submitted in partial fulfillment of the requirements for the degree of Doctor of Philosophy at the Massachusetts Institute of Technology and the Woods Hole Oceanographic Institution March 8

\begin{abstract}
This thesis addresses the issue, "Which approach to instabilities-temporal, spatial or pulse theory-is the most appropriate model for the Gulf Stream?" I also address the question of how the observations might be compared to theory. This thesis consists of two closely related parts: analytical studies that compare the three types of instability using the same realistic velocity and topography profiles; and numerical modeling that uses a continuous forcing function to examine the three types of theory in the direct context of the Gulf Stream.

\section{Analytical Studies:}

In a QG six-layer model with relatively realistic jet structure and topography, spatial instability theory gives a different and larger spatial growth rate (by about $30 \%$ ) than the equivalent spatial growth rate (ESGR) transformed from temporal instability with Gaster's formula. Faster mean flow or group velocity makes Gaster's transformation formula work better. Real parts of wavenumbers (or wavelengths) corresponding to the most temporally and spatially unstable waves are comparable and not sensitive to mean velocities, velocity shear and $\beta$. In the attempt to reconcile conflicts in the literature, it has been found that one of Hogg's (1976) conclusions-that spatially growing waves are bounded by a low wavenumber cut-off which separates them from the temporally growing waves-is not valid.

Pulse instability deals with the development of an isolated disturbance. One of the interesting features is that individual peaks in the leading edge of a wave packet can grow much faster than temporal instability predicts (Simmons and Hoskins, 1979). Because examining individual peaks is a common observational practice, this thesis focuses on how such a peak evolves. Whereas the amplitude of an individual peak increases with time, the growth rate decreases in the linear model; thus if one follows a single peak and notices a decrease in the growth rate, one cannot infer that increasing amplitude (or nonlinearity) was responsible for reducing the growth rate. In the QG six-layer model, the growth rates of individual peaks can vary from $30 \%$ to $220 \%$ of
\end{abstract}


that of the peak of the wave envelope. Direct comparison between pulse and temporal theory shows that the former allows unstable waves to have local wavenumbers beyond the short wave cut-off in the latter.

The effects of topography and vertical resolution are assessed in QG two- to six-layer models. From the analysis of potential density, it is shown that a minimum of three layers is necessary to address the effect of topography properly in the context of the Gulf Stream. In the case of pulse instability, a minimum of five layers is necessary to capture the characteristics of the instability. The effect of ageostrophy is studied by comparisons between QG and shallow-water (SW) six-layer models. The QG model overestimates the maximum growth rate by $40 \%$ compared to the $\mathrm{SW}$ model. The comparison shows that the QG model gives results quantitatively different from those from the SW model, but there is no qualitative difference between the two models in terms of the first most unstable modes. The results from both the QG and SW models have been compared to the observational analysis by Lee and Cornillon (1996a,b), and it appears to be a robust common feature in the models and the analysis that the fastest growing wave has higher growth rate, wavenumber and frequency than the most energetic wave.

\section{Numerical Experiments:}

A two-layer QG model with a jet structure and a single pulse initial condition or continuous forcing has been used to determine which type of instability theory is the most appropriate model in the context of the Gulf Stream. The first experiment shows that it takes about 45 days for a single pulse disturbance to develop close to its asymptotic form. In that period the wave packet has moved downstream $1100 \mathrm{~km}$ to about $65^{\circ} \mathrm{W}$. Hence there appears to be enough space for the pulse instability to occur in the Gulf Stream.

In the continuously forced problem, it has been found that the growth rates of individual peaks from the numerical runs scatter widely in the region from 200 $\mathrm{km}$ to $1200 \mathrm{~km}$ downstream of the forcing location. It is clear that none of the three types of instability can explain the pattern given the continuous forcing. However, the "observations" from the model runs have shown one trend predicted by pulse instability: short waves tend to move and grow faster than long waves. This cannot be predicted from temporal or spatial instability. Due to a numerical difficulty, this analysis has not been extended to the region further downstream, where the pulse instability has been found to work better in the single pulse experiment.

These numerical experiments support the robust common features of pulse instability we have studied in the analytical models, that the fastest growing wave has higher growth rate, wavenumber and frequency than the most unstable wave. 
Because the differences between the most unstable and the fastest growing waves of pulse instability theory in the analytical models seem able to explain substantial parts of the differences observed, and because the single pulse experiment suggests the analytical results may be carried over to their corresponding numerical models, pulse instability theory appears to be the most appropriate model for the Gulf Stream.

Thesis Advisor: Dr. Glenn R. Flierl

Title: Professor, Massachusetts Institute of Technology 


\section{Acknowledgments}

I sincerely thank my advisor, Glenn Flierl, for his patient guidance and encouragement over the past five and half years. In particular, I thank him for suggesting the study of pulse instability. I am extremely grateful to him for reading this thesis several times, even during weekends and holidays, and providing feedback promptly. I also thank him for his understanding and support during the past two and half years when I had to commute frequently between MA and NJ where my family has moved.

I thank the other members of my Thesis Committee: Nelson Hogg, Joseph Pedlosky, Lawrence Pratt, and Paola Rizzoli. They have been very generous with their time and expertise, and provided me with many constructive ideas. I am grateful to them for reading my defendable thesis draft in a very short time. I thank Nelson for introducing me to the paradigm of spatial instability. Joe asked many tough questions, which helped to shape the thesis. Larry often reminds me to catch the big picture before putting the pieces together. I am indebted to Paola for her continuous support and encouragement.

I thank Michael Spall for chairing my thesis defense, reading my thesis in a very short period, and making useful comments. Thanks to Professor Richard Lindzen for helpful discussions on baroclinic instability. Jochem Marotzke and Edmund Chang provided helpful comments on my thesis work. Thanks to Tong Lee for sharing the satellite data of the north wall position and for a lot of helpful discussions on observational analysis. Kyle Swanson provided his code of the saddle point finder for the study of pulse instability. Linda Meinke, Roberta Young, and Steve Meacham have lent me their helpful hands when I got into trouble in computing.

Many thanks to Ronni Schwartz, Mary Jane Tucci, Abbie Alvin and Beverly Kozol-Tattlebaum who have helped me in numerous ways. I thank Dan Burns, Stacey Frangos, Jane McNabb, Kimberly Reynolds and Helen Dietrich for their administrative support.

I thank Dr. Rui Xin Huang and his wife Lu Ping Zou for their moral support and personal advice in the past several years. Thanks also to David Chapman for introducing me to the field of oceanography and for friendship.

I thank my officemate and friend Ari Epstein and his wife Joan for many things they have done for me, including bringing me dinner when I was buried in my work. They are always ready to lend me a hand. I thank Shi Jiang and his wife Xumei Wang for a lot of enjoyable discussions on scientific and personal issues, and for the delicious dinners Xumei prepared. 
Officemates and friends Eric Won and Keith Alverson spent a lot of time polishing my English. I enjoyed very much my daily conversations with them. Huaimin Zhang and Zhengyu Liu provided me help when I stayed in Woods Hole. In the final stage of thesis writing, Jubao Zhang, Chris Edwards, Joe LaCasce, Cecily Wolfe, Molly Baringer, Alison MacDonald, and Young-Gyu Park have all helped me in different ways. Thanks also to Sarah Gille, Jim Gunson and his wife, Anne-Laure, Igor Kamenkovich, Lisan Yu and Jiayan Yang, Richard Wardle, Kate Zhang, Natalia Beliakova and many others, for their friendship. My officemate Stephanie Harrington tolerates my messing up the office in the final stage of thesis writing. I thank her for the understanding.

I thank my parents and sisters for their support and their decades of faith in me. I thank my mother-in-law, Jiping Liang, who has kindly quit her own job to take care of my son Richard while I was away from the family. I thank my father-in-law, Jinrong Dai and sister-in-law, Hong Jian Dai for their occasional help in taking care of my son. Since he was born in the middle of my endeavor, Richard has been a source of joy to my life. Above all, I owe so much to my dear wife, Hong Bin $\mathrm{Ni}$, who has suffered tremendously balancing career, family and long distance relationship while I pursued a long journey in oceanography. Without her love, understanding and support, this thesis would not have been possible.

My first three years in the Joint Program were supported by the National Science Foundation under grant OCE-9011066 and last two and half years under NSF grant OCE-9314140. 


\section{Table of Contents}

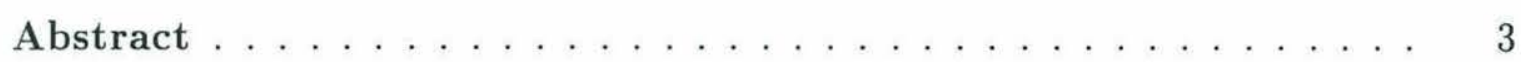

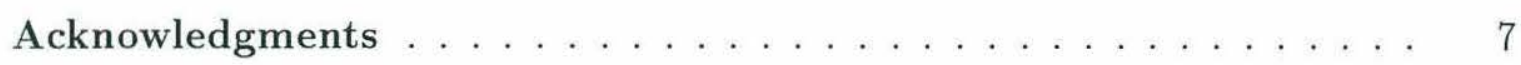

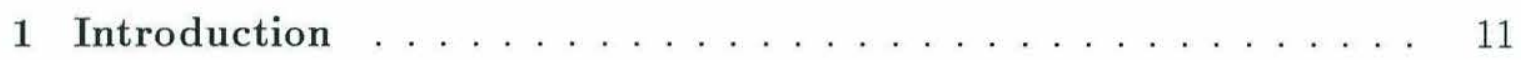

1.1 Meandering of the Gulf Stream System . . . . . . . . . . . . 11

1.2 Previous Studies and Motivation of Thesis Work . . . . . . . . 12

1.3 Plan of the Thesis $\ldots \ldots \ldots \ldots \ldots \ldots$

2 Normal Mode and Pulse Instabilities . . . . . . . . . . . . . . 19

2.1 Basic Concepts . . . . . . . . . . . . . . . . . . . 19

2.2 Model Description . . . . . . . . . . . . . . . 25

2.3 Model Results . . . . . . . . . . . . . . . . . . . . . . . . 27

2.4 Comparison between the three types of instability theories . . . . 54

2.5 Numerical Experiments $\ldots \ldots \ldots \ldots \ldots$

2.6 Summary . . . . . . . . . . . . . . . . . . . . . . . . 79

3 The Instabilities of the Gulf Stream-QG Models . . . . . . . . . 83

3.1 Motivation . . . . . . . . . . . . . . . 83

3.2 Model Description . . . . . . . . . . . . . . . . . 85

3.3 Basic Flow and Parameter Calibration in Multilayer Models . . . . 87

3.4 Comparison Among Multilayer Models . . . . . . . . . . . . 97

3.5 Temporal, Spatial and Pulse Instabilities in a Six-Layer Model . . . 100

3.6 Summary and Conclusions . . . . . . . . . . . . . . . . . 109

4 Shallow-Water Models . . . . . . . . . . . . . . . . . . . . 113

4.1 Motivation . . . . . . . . . . . . . . . . 113

4.2 Model Description . . . . . . . . . . . . . . . . . . . 114 
4.3 Comparison of the SW and QG Six-Layer Models . . . . . . . . . 118

4.4 Comparisons With Observations and Conclusion . . . . . . . . 125

5 The Instabilities of the Gulf Stream-Numerical Simulation . . . 129

5.1 Introduction . . . . . . . . . . . . . . . . . 129

5.2 A Two-Layer QG Analytical Model . . . . . . . . . . . . . . . 130

5.3 Temporal, Spatial and Pulse Instabilities in a Two-Layer Model . . . 135

5.4 A Two-Layer QG Numerical Model . . . . . . . . . . . . . . . . . . 143

5.5 Development of a Single Pulse . . . . . . . . . . . . . . . . . 148

5.6 Continuously Forced Problem . . . . . . . . . . . . . . . . . . 164

5.7 Summary and Discussion . . . . . . . . . . . . . . . 177

6 Summary and Conclusion . . . . . . . . . . . . . . 181

6.1 Analytical Studies . . . . . . . . . . . . . . . . . . . 181

6.2 Numerical Experiments . . . . . . . . . . . . . . . . 186

6.3 Limitation of This Study and Future Work . . . . . . . . . . . . 188

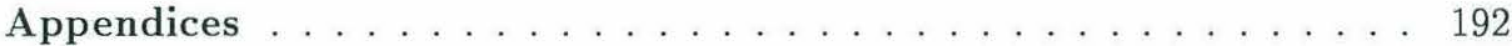

A The Saddle Point Finder . . . . . . . . . . . . . . . . . 192

B Method for Solving Spatial Instability Problem . . . . . . . . . 195

C Effects of Resolution and Channel Width . . . . . . . . . . . . 196

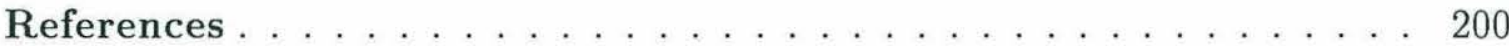




\section{Chapter 1 \\ Introduction}

\subsection{Meandering of the Gulf Stream System}

The Florida Current, after it leaves the Florida Straits, develops meanders along its path downstream. Beyond Cape Hatteras the meanders develop to large amplitudes. In the past three decades, many analytical and numerical models have been developed to explain these meanders as the results of baroclinic and barotropic instabilities. The Gulf Stream system, however, is too complicated for a single dynamic model to incorporate most of its main features. The complexity results from the following factors. First, the topography and coast have significant effects on the path and instability of the system (Warren, 1963, Orlanski, 1969). Second, the along stream variation of mean fields as well as that of the topography affects the development of the meanders. Third, the well-developed and studied quasi-geostrophic models may be inappropriate to handle the significantly large slope of mean density fields or the large Rossby number of the flow. Fourth, nonlinearity may have an important effect on the evolution of the meanders. Moreover, the classic theories of normal mode instability only provide the temporal growth rates that cannot be compared directly with the observed growth rates of individual wave peaks, which are often part of nonperiodic wave patterns. 


\subsection{Previous Studies and Motivation of Thesis Work}

In this section, I will list some of previous studies related to the Gulf Stream instability in Table 1 and 2. I will review the main results of a few most relevant papers to provide the background and to build up the motivation for this thesis work.

\section{Summary of Literature Survey:}

Table 1: Most relevant Papers

\begin{tabular}{|l|l|c|c|c|c|}
\hline Author & Model char. & Dimen. & Type of inst. & Topog. & Comment \\
\hline \hline Orlanski 1969 & PE 2-Layer & 2D & mixed & yes & \\
\hline Holland et al 1980 & QG 2-Level & 2D & mixed & no & \\
\hline Johns 1988 & QG cont. & 1D & baroclinic & yes & \\
\hline Kontoyiannis 1992 & QG cont. & 2D & mixed & yes & \\
\hline Xue 1991a,b & PE cont. & 2D, 3D & mixed & yes & \\
\hline Orlanski et al1973 & PE cont. & 3D & baroclinic & yes & ivp \\
\hline Luther et al 1985 & PE cont. & 3D & mixed & yes & ivp \\
\hline
\end{tabular}

L : Layer

PE: Primitive Equations

SW: Shallow-Water Eqns

ivp: initial value problem

Topog.: Topography

Orlanski (1969) studied the stability of a two-layer model with bottom topography in the context of the Gulf Stream. Applying two different types of bottom topography typical in the regions upstream and downstream of Cape Hatteras, he found that the most unstable disturbances, corresponding to the basic flow upstream from Cape Hatteras, are markedly different in wavelength and period from those corresponding to the basic flow downstream from Hatteras. This suggests the importance of bottom topography on unstable waves. However, Orlanski used simple analytical forms for topography; one effort of this thesis is to assess the effect of more realistic 
topography and better vertical resolution in the context of the Gulf Stream beyond Cape Hatteras.

Table 2: Other relevant Papers

\begin{tabular}{|c|c|c|c|c|c|}
\hline Author & Model char. & Dim. & Type of inst. & Topog. & Comment \\
\hline Lipps & $\mathrm{QG} 1 \frac{1}{2} \mathrm{~L}$ & $2 \mathrm{D}$ & equiv. bt. & no & $\beta$-plane \\
\hline Tareyev & QG cont. & $2 \mathrm{D}$ & mixed & no & \\
\hline Sela et al & $\mathrm{PE} 1 \frac{1}{2} \mathrm{~L}$ & $2 \mathrm{D}$ & equiv. bt. & no & ageostr. \\
\hline Abramov et al'72a & PE 2-Layer & $2 \mathrm{D}$ & baroclinic & no & $\beta$-plane \\
\hline Abramov et al'72b & QG 2-Layer & $2 \mathrm{D}$ & mixed & no & \\
\hline Nikitin et al 1972 & PE 2-Layer & $2 \mathrm{D}$ & baroclinic & yes & $\beta$-plane \\
\hline Hart & QG 2-Layer & $2 \mathrm{D}$ & mixed & no & \\
\hline Flierl $\quad 1975$ & QG 1-L/2-L & $2 \mathrm{D}$ & bt.,bc./mixed & no & $\beta$-plane \\
\hline Abramov et al '83 & QG 2-Layer & $2 \mathrm{D}$ & mixed & yes & $\beta$-plane \\
\hline $\begin{array}{ll}\text { Ikeda } & 1983\end{array}$ & QG 3-Layer & $2 \mathrm{D}$ & mixed & yes & \\
\hline Talley & QG 1-Layer & 1D & baroclinic & no & $\beta$-plane \\
\hline Talley & QG 2-Layer & $2 \mathrm{D}$ & mixed & no & $\beta$-plane \\
\hline Killworth $\quad 1980$ & QG 2-L/cont. & $2 \mathrm{D}$ & mixed & no & $\beta$-plane \\
\hline Killworth et al '82 & QG $1 \frac{1}{2} \mathrm{~L}$ & $2 \mathrm{D}$ & equiv. bt. & no & coastal front \\
\hline Paldor $\quad 1983 a$ & QG $1 \frac{1}{2} \mathrm{~L}$ & $2 \mathrm{D}$ & equiv. bt. & no & coastal front \\
\hline Paldor & QG $1 \frac{1}{2} \mathrm{~L}$ & $2 \mathrm{D}$ & equiv. bt. & no & density front \\
\hline Killworth 1983 & QG $1 \frac{1}{2} \mathrm{~L}$ & $2 \mathrm{D}$ & equiv. bt. & no & density front \\
\hline Killworth et al '84 & QG 2-Layer & $2 \mathrm{D}$ & baroclinic & no & density front \\
\hline Barth $\quad 1987$ & SW 2-Layer & $2 \mathrm{D}$ & mixed & no & coastal front \\
\hline Paldor et al 1991 & QG 2-Layer & $2 \mathrm{D}$ & mixed & no & coastal front \\
\hline Flierl preprint & QG 2-Layer & $2 \mathrm{D}$ & mixed & no & contour dyn. \\
\hline
\end{tabular}

Johns (1988) considered 1-D baroclinically unstable waves on the Gulf Stream PV gradient near Cape Hatteras. This linear instability model includes the topography variation in the cross-jet direction, however it excludes the cross-jet variation of basic flow, stratification and PV by averaging them across the jet and hence eliminates the barotropic instability. The model calculation results in a temporal growth rate $k c_{i}$, which is then transformed into an equivalent spatial growth rate (ESGR), using $K_{i}=k c_{i} / C_{g}$, where $k$ is the along stream wavenumber and $C_{g}$ is the group velocity. This continuously stratified model was successful in predicting the time and 
length scales of growing meanders, but predicted a smaller growth rate than that observed by a factor of two.

Two major approximations were employed in Johns' model. The first is that the basic flow does not have cross-jet variation. This neglect of the variation may cause significant error as it was first pointed out by Phillips (1964). Moreover, because the thermocline is located at different depth across the stream, averaging the flow horizontally across the jet will effectively reduce the vertical gradient of basic flow across the thermocline. Hence this approximation tends to decrease the growth rate. The second is the QG assumption, which assumes small Rossby number. This is not a good assumption because the observations show that Rossby number is from 0.3 to 0.5 in the Gulf Stream (Bower, 1989). Orlanski (1969) has found that the finite Rossby number decreases the instability. Hence the effect of this approximation is to increase the growth rate. The two assumptions therefore have competing effects on instability, and they may cancel each other partially or totally. When this model was extended to 2-D (i.e., barotropic instability recovered) by Kontoyiannis (1992, $\mathrm{Ph}$. D. thesis, and 1994) the equivalent spatial growth rate of a mainly baroclinic mode became comparable to that observed. However, he used phase velocity instead of group velocity in the transformation (giving an estimate $\omega_{i} / C_{p h}$ which we will call the "pseudo-equivalent spatial growth rate", PESGR), which increased the equivalent spatial growth rate by a factor ranging from $5 / 3$ (long waves) to 6 (short waves). The comparison would have been much worse if group velocity had been used instead of phase velocity in transforming the growth rate.

Xue (1991a, thesis) applied a 2D primitive equation analytical model to study temporal instability of the Gulf Stream. The model also included the effect of bottom topography and gave results which were in good agreement with observations in terms of periods and wavelengths. She extended the study in a $3 \mathrm{D}$ numerical model (Xue, 1991b, thesis) in order to investigate whether the unstable waves in 
the $2 \mathrm{D}$ model will be able to grow into finite amplitude meanders before the mean condition of the Gulf Stream changes significantly and whether the result in the 2D model will be altered considerably if spatially growing modes are also permitted as well as temporally unstable modes. She estimated a growth rate of the dominant wave in the model from the change of eddy kinetic energy, but did not resolve the full dispersion relation. The estimated growth rate was not compared to the observations directly; nor was the result not analyzed in terms of either of three approaches to instabilities discussed below. Thus it is hard to infer from these simulations which is the most appropriate approach for the Gulf Stream meandering.

Luther and Bane (1985) present a numerical model study of the unstable normal modes of oscillation of the Gulf Stream. Their model uses the primitive equations and has filtered out all other types of instabilities except spatial instability. They found that one of modes from the model produces many of the features of an observed 8-day wave, e.g., comparable phase speed, current and temperature structures. However they did not compare the model growth rates with observations. Our study has showed that the differences among temporal, spatial and pulse instabilities are much more prominent in growth rates than in phase speeds and other structures (see Chapter 2 and 3). So it is not obvious that spatial instability studied in their model is the best explanation for the meanders.

All the studies summarized above except Luther and Bane's (1985) and Xue (1991b) considered dynamically only temporally unstable modes. Gaster (1962) showed that for small rates of amplification, to the lowest order of approximation, the frequency for a disturbance growing with respect to time is the same as that of a spatially growing wave with the same wavenumber, and the spatial growth rate is related to the temporal growth rate by the group velocity, as I discussed above. Hogg (1976) found that spatial and temporal waves in a baroclinic model are separated in wavenumber with short waves being spatially unstable and long waves being 
temporally unstable. A neutral wave separates the two cases. This suggests that the transformation formula does not hold in that particular case at least. In fact, the validity of Gaster's formula has not yet been studied in detail in a realistic oceanic context and the relation between temporal and spatial growth is not well understood. Many of the authors mentioned above consider that spatial instability is more relevant than temporal one in the context of the Gulf Stream (e.g. Johns, 1988, Xue, 1991a,b); however, spatially unstable modes were not studied in their models because of the difficulty of "nonlinearity" (the eigenvalue problem for spatial growth rate is not linear; see Section 2.3.2). Some authors regarded both temporal and spatial instabilities as the results of the same instability mechanism (e.g. Thacker, 1976), while others considered them as different kinds of unstable modes (e.g. Hogg, 1976, Luther and Bane, 1985). It appears interesting and meaningful to make this relation clear. And this is the first goal of the thesis.

Both temporal and spatial instability theories are highly idealized. The temporal instability paradigm assumes that disturbances are distributed periodically in the along stream direction, while spatial instability assumes a periodic forcing with a single frequency (see Section 2.3) and hence a single dominant wavenumber over the whole domain. These assumptions are far from being realistic. In the context of Gulf Stream meandering, it is more appropriate to solve an initial value problem or a locally forced problem. If the initial disturbance is localized in a relatively small region, it will be simpler to consider pulse instability instead of the most general initial value problem.

Many studies (e.g., Boudra et al,1988, Xue,1991b) suggest it to be most likely that the frontal disturbances are initiated and grow in the Florida Straits. Xue (1991b) concludes that her model is able to produce realistic meanders only when it is forced at the southern boundary, and meanders entering the domain are amplified downstream of the Charleston Bump. Observations show that frontal meanders of 
the Gulf Stream are not significantly correlated to any apparent forcing, such as local wind stress, in the South Atlantic Bight (Lee and Waddell, 1983). Many authors believe that initial disturbances are introduced in some special small regions such as the Florida Straits, the Charleston Bump, and Cape Hatteras, and are amplified when they propagate down stream. For this reason, I will also study the development of locally forced disturbances.

Pulse instability allows wave amplitudes and wavenumbers to change in the space (see Section 2.3) and hence is less idealized and restrictive than the temporal and spatial instabilities. However the assumption of a single pulse disturbance and no further forcing has its own limitations. In the context of the Gulf Stream, it is more likely that the system is forced continuously. To determine which type of the instabilities is the most appropriate simple model in this complicated context, I will study a QG two-layer numerical model in Chapter 5.

Lee and Cornillon (1996) have found that the most energetic meanders in the Gulf Stream have a period of 46 days and a wavelength of $427 \mathrm{KM}$, while the fastest growing meanders have a period of 40 days and a wavelength of $350 \mathrm{KM}$. These differences can not be explained by classic normal mode instability models. The second goal of this thesis is to provide a dynamic explanation for the discrepancy.

The applicability of the QG model in the study of Gulf Stream meandering has long been questioned. Since QG models formally apply only in the case of small Rossby number, and small slopes of isopycnals and topography, we need to examine first whether these conditions have been violated in the Gulf Stream. From the observations, the Rossby number was estimated to be .3-.5 in the Gulf Stream east of Cape Hatteras (Bower, 1989). The depth of the top of the thermocline varies from around $800 \mathrm{~m}$ in Southern side to about $200 \mathrm{~m}$ in Northern side, and the depth of the bottom changes from about $4900 \mathrm{~m}$ to $1900 \mathrm{~m}$ across a CTD section at $68^{\circ} \mathrm{W}$ running from $36.3^{\circ} \mathrm{N}$ to $40^{\circ} \mathrm{N}$ (Hall and Fofonoff, 1993). Such large changes in the depths of 
isopycnals and topography violate the formal assumptions of QG theory. Therefore one may expect that the QG approximation is not appropriate in the context of the Gulf Stream, and a question naturally arises whether the results obtained in QG models are qualitatively similar to primitive equation or shallow-water models and how large the quantitative differences are. To answer this question, I devote one chapter (Chapter 4) to the comparison between QG and Shallow-Water models (SW). This becomes the third goal of the thesis.

\subsection{Plan of the Thesis}

In Chapter 2, I will first introduce the basic concepts of temporal, spatial and pulse instabilities. I then start with a simple two-layer QG model with uniform velocities on each layer, Phillips' model, to study and compare the characteristics of these types of instabilities. This study is then extended to a QG six-layer model with more realistic velocity profile resembling the Gulf Stream in Chapter 3. The

effect of bottom topography is also considered in the model. In Chapter 4, I will develop a shallow-water (SW) six-layer model to investigate the effect of ageostrophy. Comparisons of QG and SW models with observations will be made in Chapter 4 . In Chapter 5, I will run some numerical experiments with relatively realistic velocity profiles and forcing to see which type of instability theory is most appropriate for the Gulf Stream and which are likely to be picked out by common observational approaches. Summaries and conclusions are presented in Chapter 6. 


\section{Chapter 2}

\section{Normal Mode and Pulse Instabilities}

\subsection{Basic Concepts}

\subsubsection{Normal mode, temporal, and spatial instabilities}

Since the pioneering studies of Charney (1947) and Eady (1949), temporal instabilities in quasi-geostropic systems have been investigated extensively. Charney and Stern (1962) developed stability criteria for the case of an internal jet, i.e., where the meridional temperature gradients at the ground vanish. Pedlosky $(1963,1964)$ found the stability criteria for more general cases incorporating the effects of surface temperature gradient and topography and also found bounds on phase speed and growth rate. These studies contributed to form a more general stability criterion commonly known as the Charney-Stern stability theorem.

In studies of temporal instabilities, normal mode solutions of the form $\exp (i(k x-\omega t))$ are investigated. More exactly, real wavenumber $k$ and complex $\omega$ are assumed in the above expression. Alternatively, we may take $\omega$ to be real and the wavenumber to be complex. This will lead to another type of normal mode instability, spatial instability. There is also another alternative where both $k$ and $\omega$ are complex.

Linear spatial instability was first studied by Michalke (1965) in a barotropic flow with a hyperbolic tangent velocity profile. He calculated the growth rates and phase speeds numerically from temporal (using PESGR) and spatial instability theories, and compared them with experimental results of Freymuth (1965). The experiments tended to support spatial instability at low forcing frequency and to agree with temporal instability at high frequency. At higher frequency, the equivalent spa- 
tial growth rate converted from temporal instability, the PESGR, was found to be lower at high frequencies. Michalke estimated that nonlinearity reduced the growth rate more at higher frequencies and speculated that the tendency to approach the PESGR at high frequency was due to nonlinearity.

Although both temporal and spatial instabilities are types of normal mode instability, the relation between them is nevertheless obscure. In the context of Gulf Stream instabilities, numerous studies have been done of temporal instabilities whereas only a few have examined spatial instabilities. In papers on the temporal instabilities of Gulf Stream, many authors (Xue, 1991, Johns, 1988, etc.) agree that spatial instability is probably more relevant than the temporal one in the context. However the study of spatial instability is more difficult than that of temporal one because the linearized QG equations lead to a non-standard eigenvalue problem whose matrix contains nonlinear terms of wavenumber $k$ when we fix frequency (which is assumed to be real) and solve for wavenumber (see section 2.3.2). Spatial instability corresponds to a system forced in a local region at a constant frequency, e.g. by a wavemaker. Hogg (1976) solves for complex $k$ for a given phase speed, $c$, rather than a given real frequency, which rendered the problem a linear eigenvalue one. However it is not easy to justify a fixed phase speed instead of a fixed real frequency condition in realistic systems since both the frequency and wavenumber are then required to be complex, and mechanisms which could fix the phase speed are unknown. I will return to this issue in Section 2.3.2.

Observations and data analysis normally provide growth rates of individual peaks by peak-tracking techniques. How can a model of temporal instability be compared to observations? A relation proposed by Gaster (1962) is frequently used to convert the growth rate of temporal instability to an equivalent spatial growth rate. However Gaster's transformation formula is only valid when growth rate is small. Is the growth rate in Gulf Stream instability problem small enough to use the formulae? 
As I mentioned above, Michalke (1965) showed in his problem that the difference between temporal and spatial instabilities is not very large (the pseudoequivalent spatial growth rate, transformed from the temporal growth rate divided by phase speed, is only $17 \%$ smaller than that from spatial instability theory). Such a result is not, of course, general; in the Gulf Stream context of a baroclinic jet on the beta-plane, the differences between the various estimators of spatial growth rate are not known. They will be examined in detail in the next chapter.

\subsubsection{Pulse, absolute and convective instabilities}

Normal mode instability theories, temporal and spatial, assume that the disturbance is periodic in $x$ or $t$ respectively. These assumptions limit their capability in explaining the development of localized disturbances. Another type of linear solution - pulse instability - may be more relevant in this case. Pulse instability represents the asymptotic solution to the evolution of a localized disturbance, as an approximation to the full initial value problem as $t \rightarrow \infty$. The full IVP usually requires the use of a Fourier transform in space and a Laplace transform in time and eventually double inverse transforms. This in general is a formidable task even for the simplest of models. However, if we are only concerned about the asymptotic behavior of the evolution, it is appropriate to consider pulse instability.

Pulse instability may be further divided into two categories - "absolute" and "convective" instabilities. These two concepts have been known in plasma physics for a long time and are helpful in explaining the evolution of a localized wave packet. The concepts can be easily illustrated in Figure 2.1 (from Briggs, 1964). When absolute instability happens, the disturbance spreads out in both directions so that the disturbance keeps growing in time wherever the disturbance reaches. On the other hand, when convective instability occurs, the disturbance "propagates along" 
the system as it grows in time so that it eventually disappears if one stands at a fixed point.

When a system is convectively unstable and is continously forced with constant frequency at a local region, spatial instability may occur. Spatial instability, as described above, assumes real $\omega$ and complex $k$. It also presumes an origin $x=0$ where the system has fixed amplitude and frequency, e.g. where a wavemaker generates disturbances at a constant frequency. When the system is convectively unstable, disturbances generated at early times propagate away from the forcing location so that the amplitude at the point is closely related to the amplitude of the forcing. This makes the condition 'fixed amplitude' at the point more easily satisfied and spatial instability more likely to occur. On the other hand, if the system is absolutely unstable instead, the growing waves at the forcing location may eventually dominate the forcing amplitude, which makes it improbable that the amplitude at the point will be constant, and unlikely that spatial instability will give a reasonable description. 


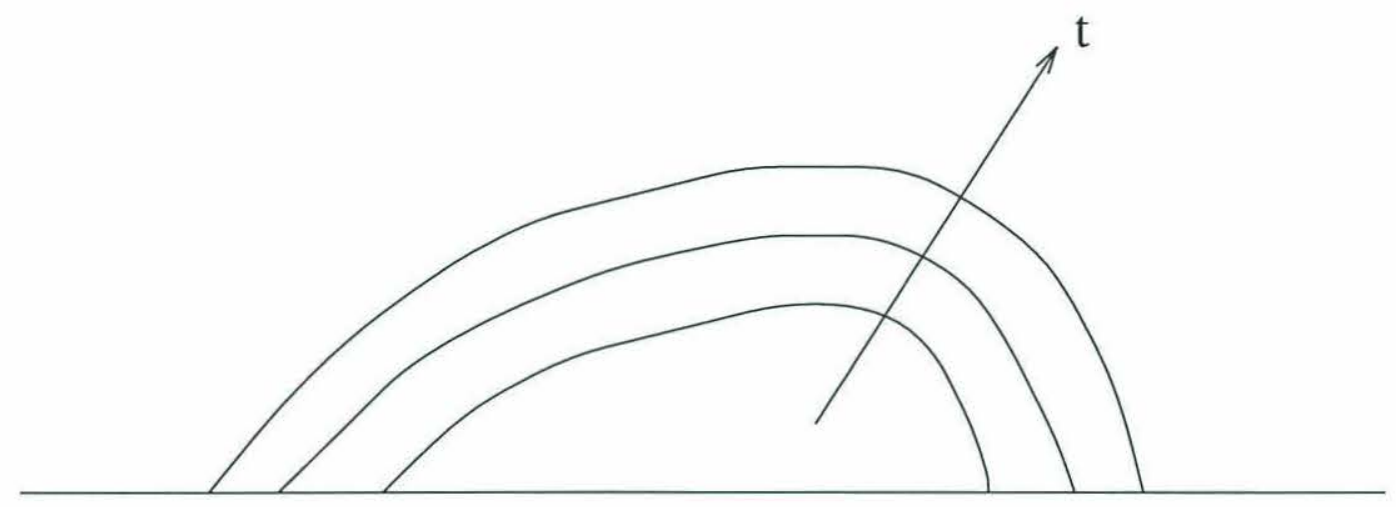

\section{Absolute Instability}

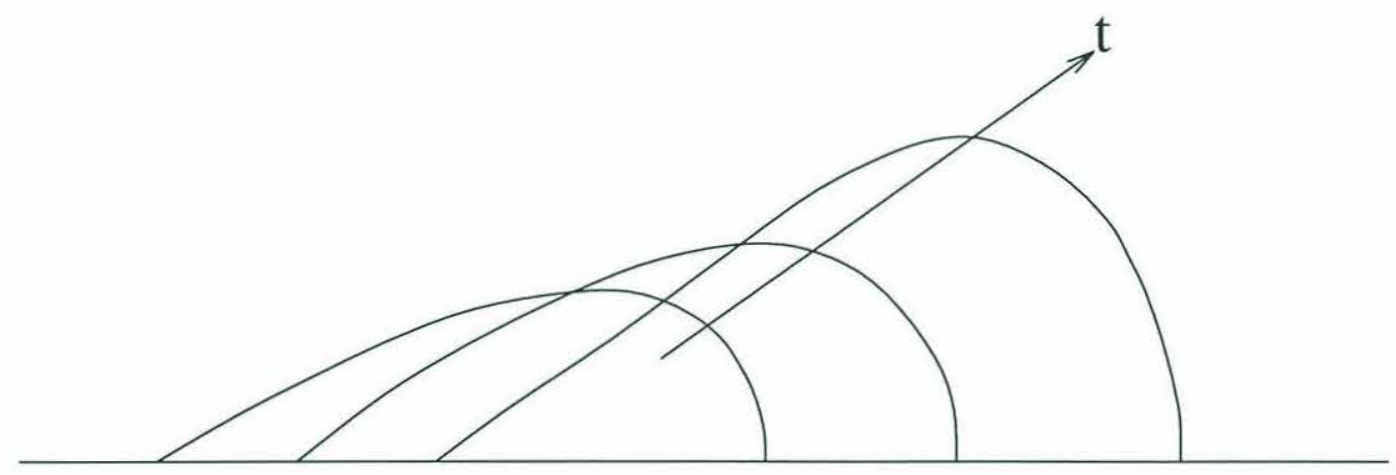

\section{Convective Instabilitv}

Figure 2.1: Upper panel: absolute instability. Lower panel: convective instability. 


\section{Amplitude}

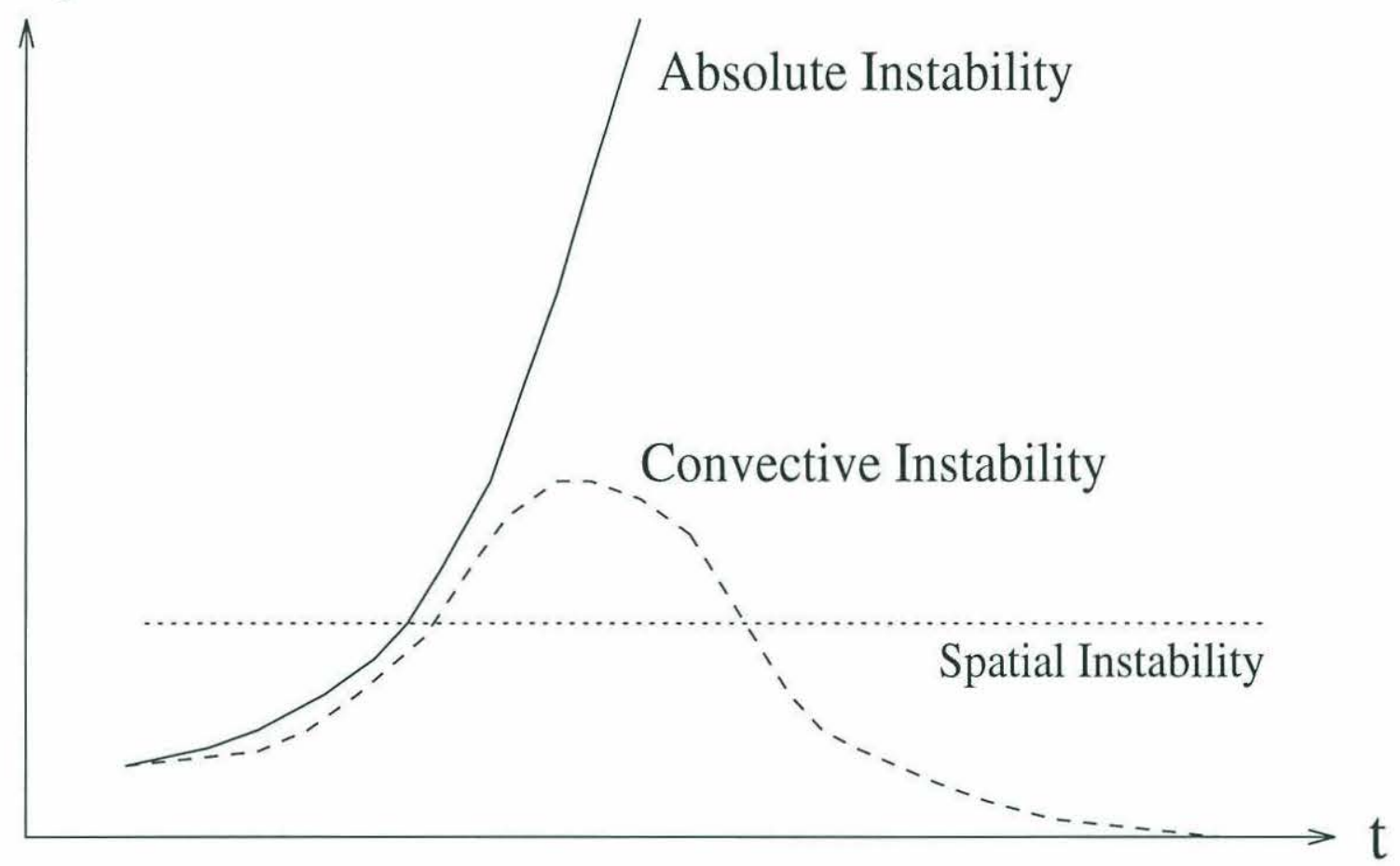

Figure 2.2: Absolute, Convective and Spatial instability. 


\subsection{Model Description}

We start with a simple two-layer quasi-geostrophic channel model (Phillips, 1954, Pedlosky, 1986) to demonstrate the concepts of temporal, spatial, pulse, absolute, convective instabilities. Using such a simple model will allow us to focus on the basic conceptual ideas. We will solve the linear instability problem analytically and then run a numerical model with the same sets of governing equations to study how initial disturbances evolve into these idealized types of instabilities.

The model does not consider friction. The stream function in each layer is independent of depth. Let $n=1$ indicate the upper layer, and $n=2$ the lower one. Following Pedlosky (1986, Sec. 7.9 to 7.11 ), the equation of motion can be written

$$
\left[\frac{\partial}{\partial t}+\frac{\partial}{\partial x} \psi_{n} \frac{\partial}{\partial y}-\frac{\partial}{\partial y} \psi_{n} \frac{\partial}{\partial x}\right]\left[\nabla^{2} \psi_{n}-F_{n}(-1)^{n}\left(\psi_{2}-\psi_{1}\right)+\beta y\right]=0
$$

with boundary condition

$$
\psi_{n}=0, \quad y=0, L_{y}, \quad n=1,2
$$

where $\beta$ is the nondimensional gradient of planetary vorticity and $F_{n}$ the Froude number in each layer, i.e.

$$
\begin{array}{r}
\beta=\beta_{\circ} \frac{L^{2}}{U} \\
F_{n}=\frac{f_{o}{ }^{2} L^{2}}{g\left[\left(\rho_{2}-\rho_{1}\right) / \rho_{\circ}\right] H_{n}}
\end{array}
$$

$L$ is the length scale of motion we are focusing on and $H_{n}$ the depth of each layer. The standard linear instability theory for a basic state with uniform zonal flows $U_{n}$ and perturbations $\phi_{n}=A_{n} \sin \left(l_{j} y\right) e^{i k(x-c t)}$ gives the eigenvalue problem

$$
\operatorname{det}\left|\begin{array}{l}
\left(c-U_{1}\right)\left(K^{2}+F_{1}\right)+\beta+F_{1}\left(U_{1}-U_{2}\right), \quad-\left(c-U_{1}\right) F_{1} \\
-\left(c-U_{2}\right) F_{2}, \quad\left(c-U_{2}\right)\left(K^{2}+F_{2}\right)+\beta+F_{2}\left(U_{2}-U_{1}\right)
\end{array}\right|=0
$$

where $K$ is the total wave number,

$$
K^{2}=k^{2}+l_{j}^{2}
$$




$$
l_{j}=\frac{j \pi}{L_{y}} \quad j=0,1,2, \ldots
$$

For given $k$ or $k^{2}$, Equation (2.2.4) is quadratic for $c$ or $\omega$. And for given $c$, it is also quadratic for $k^{2}$ or $K^{2}$. Thus both temporal instability and the case of spatial instability for given $c$ considered by Hogg (1976) have simple solutions. In more general cases with more layers and/or multiple grid points in $y$, these two cases can still be expressed as standard eigenvalue equations for $c$ or $k^{2}$. However for given $\omega=k c$, Equation (2.2.4) becomes a sixth order equation for $k$ (see section 2.3.2). Therefore even the simple Phillips' model does not have a simple analytical solution for $k$ given $\omega$. In the more general case, the eigenvalue problem cannot be expressed in standard form. The dispersion relationship Equation (2.2.4) will be analyzed in Section (2.3) using the three approaches-temporal, spatial, and pulse instabilities. 


\subsection{Model Results}

This section will represent solutions to Equation (2.2.4) in subsections depending on the approaches.

\subsubsection{Temporal Instability}

The temporal instability approach assumes real wavenumber $k$ and solves for complex frequency $\omega$ or phase speed c. Equation (2.2.4) gives a quadratic equation for $c$, whose solutions are discussed in detail by Pedlosky (1986, Section 7.11)

$$
\begin{array}{r}
c=U_{2}+\frac{U_{s} K^{2}\left(K^{2}+2 F_{2}\right)-\beta\left(2 K^{2}+F_{1}+F_{2}\right)}{2 K^{2}\left(K^{2}+F_{1}+F_{2}\right)} \\
\pm \frac{\left[\beta^{2}\left(F_{1}+F_{2}\right)^{2}+2 \beta U_{s} K^{4}\left(F_{1}-F_{2}\right)-K^{4} U_{s}{ }^{2}\left(4 F_{1} F_{2}-K^{4}\right)\right]^{1 / 2}}{2 K^{2}\left(K^{2}+F_{1}+F_{2}\right)}
\end{array}
$$

Here we only take an example to show how the solutions look like for a set of parameters tuned to oceanic cases where the ratio of the upper layer depth to the lower one is normally taken to be $1 / 5$, hence $F_{1}$ and $F_{2}$ are chosen to be $5 / 6$, 1/6. Two sets of parameters for $U_{1}$ and $U_{2}$ were used: $(1.3,0.5)$ and $(1.0,0.2)$. The velocity shear between two layers is kept constant so that we can investigate how the mean velocity affects the nature of instabilities. $\beta$ is arbitrarily set to be 0.05 for most cases. $\beta$ can be varied to change the growth rates of the instabilities. For a set of parameters $\left(U_{1}=1.0, U_{2}=0.2, \beta=.05\right)$, the solutions are shown in Figure 2.3.

\subsubsection{Spatial Instability}

Spatial instability is achieved when a system is forced at a local region with a constant frequency. For a given $\omega=k c$, Equation (2.2.4) can be rewritten as 

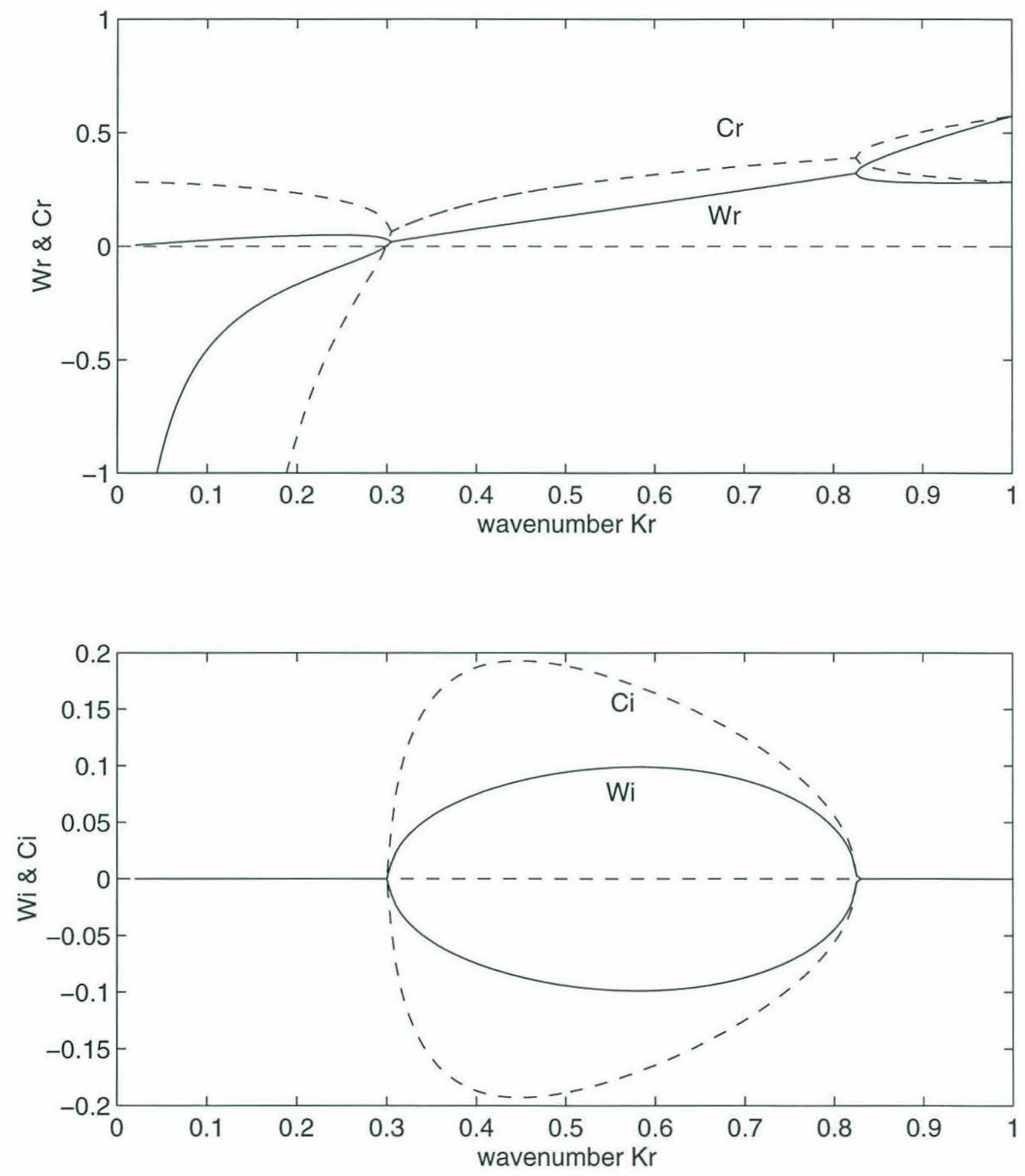

Figure 2.3: Upper panel: real frequency $\omega_{r}$ and phase speed $c_{r}$ vs k,solid line: $\omega_{r}$, dashed line: $c_{r}$. Lower panel: $\omega_{i}$ and $c_{i}$ vs $\mathrm{k}$, solid line: $\omega_{i}$, dashed line: $c_{i}$ 


$$
\operatorname{det}\left|\begin{array}{lr}
\left(\omega-k U_{1}\right)\left(K^{2}+F_{1}\right)+k \beta+k F_{1}\left(U_{1}-U_{2}\right), \quad-\left(\omega-k U_{1}\right) F_{1} \\
-\left(\omega-k U_{2}\right) F_{2}, \quad\left(\omega-k U_{2}\right)\left(K^{2}+F_{2}\right)+k \beta+k F_{2}\left(U_{2}-U_{1}\right)
\end{array}\right|=0
$$

However this gives a sixth order algebraic equation in $k$ for any given $\omega$. Its solution can only be calculated numerically.

For comparison, the parameters $F_{n}$ and $U_{n}$ are chosen to be the same as in the temporal instability case. The solutions are shown in Figure 2.4.

In the upper panel of Figure 2.4, there are six solutions for each given real $\omega$ (some solutions may have the same real parts or the same zero imaginary parts). Two solutions have large values of $\left|k_{i}\right|$ which do not vanish when $\omega$ goes to $\infty$. These solutions should be discarded according to a "rule" due to Briggs (1964). The criterion basically says that for given $\omega_{r}=\omega_{0}$, if we increase $\omega_{i}$ from 0 to $\infty$, only those solutions of $k$ whose imaginary parts are negative when $\omega_{i}=0$ and change their signs when $\omega_{i}$ increases correspond to the growth of the disturbance. The physical idea behind this rule is that all growing waves have finite phase speeds and growth rates - which means that signals propagate and grow with some delay with respect to the forcing source. If we let the amplitude of the forcing grow at a rate, say $\omega_{i}$, waves in the far field will respond to the forcing amplitude at some previous time when it was weaker. And if we increase $\omega_{i}$ from 0 to $\infty$, the spatially growing waves which exist when $\omega_{i}=0$ will eventually become small compared to those near the forcing source, i.e. the forcing grows faster than waves in the far field. Thus the disturbance looks like decaying waves. On the other hand, waves which are decaying when $\omega_{i}=0$ will remain as decaying waves and hence their spatial growth rates will never change signs when $\omega_{i}$ increases from 0 to $\infty$. In the following I will take an example to show how to apply this criterion.

Let us take $\omega_{0}=\omega_{r}=.2$ and we calculate $k$ as a function of $\omega=\omega_{r}+i \omega_{i}$. As $\omega_{i}$ runs from 0 to $\infty$ (or to a number large enough), we keep track of the solutions 

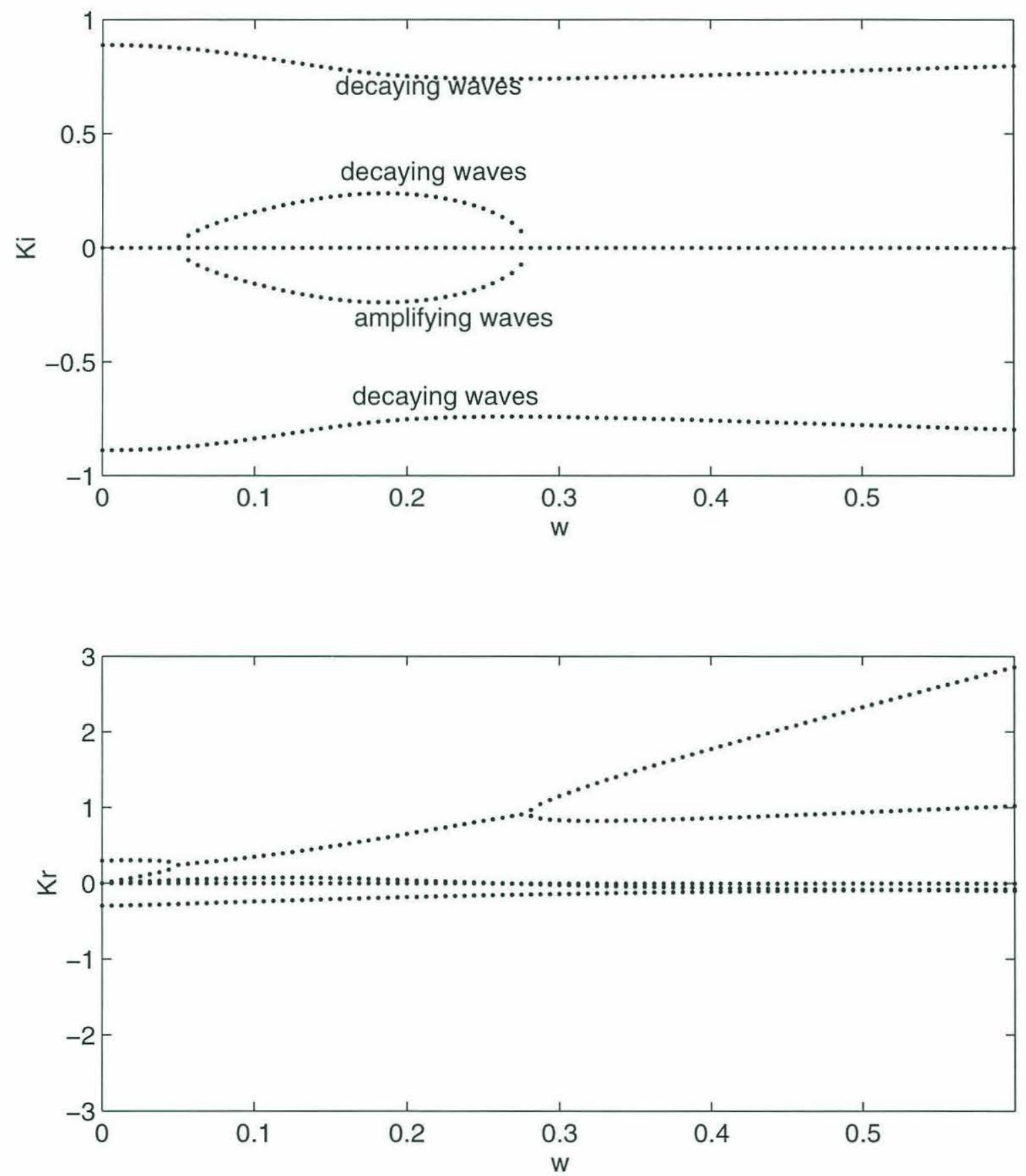

Figure 2.4: Upper panel shows the spatial growth rates as functions of frequency. The dotted curve with largest negative $k_{i}$ does not correspond to an amplifying wave as explained in the text. The lower panel shows the real wavenumbers as functions of frequency. 
of $k$. Figure 2.5 shows the evolution of $k$ as $\omega_{i}$ increases. The starting point of each solution corresponds to $\omega_{i}=0$. Only one solution crosses the real axis of $k$ and also has a starting point below real axis of $k$, corresponding to the spatial growing mode. we will see later on page 78 in Section 2.5.1 that this mode is the only growing wave we can observe in numerical runs.

Our analysis above assumes that disturbances are generated at $x=0$ and are propagating in the positive $x$ direction. This requires negative $k_{i}$ for the disturbance to be spatially unstable. For the convenience of comparison, we often compare $-k_{i}$ with estimates of spatial growth rates.

In the same paper mentioned above by Briggs (1964), he also proved that for any system to support unstable waves, its dispersion equation must yield complex solutions with positive imaginary parts for some real $k$, i.e. it is a necessary condition for any unstable waves.

In contrast, Hogg (1976) argued that the wavenumber can be complex for real phase speed in the limit of zero frequency and his analysis implied that spatial instability does not require potential vorticity gradients to change sign. However, he did not consider Brigg's criterion (this was first pointed out by Pierrehumbert, 1986) and so may have included complex $k$ roots which do not correspond to actual spatial instabilities. In addition, the purely spatially unstable wave Hogg considered is really better thought of as neutral because its phase speed is real. This can be seen by following an individual peak of the wave.

$$
\begin{aligned}
A_{0} e^{i(k x-\omega t)} & =A_{0} e^{i k(x-c t)} \\
& =\left[A_{0} e^{-k_{i}(x-c t)}\right] e^{i k_{r}(x-c t)} \\
& =A_{1} e^{i k_{r}(x-c t)}
\end{aligned}
$$




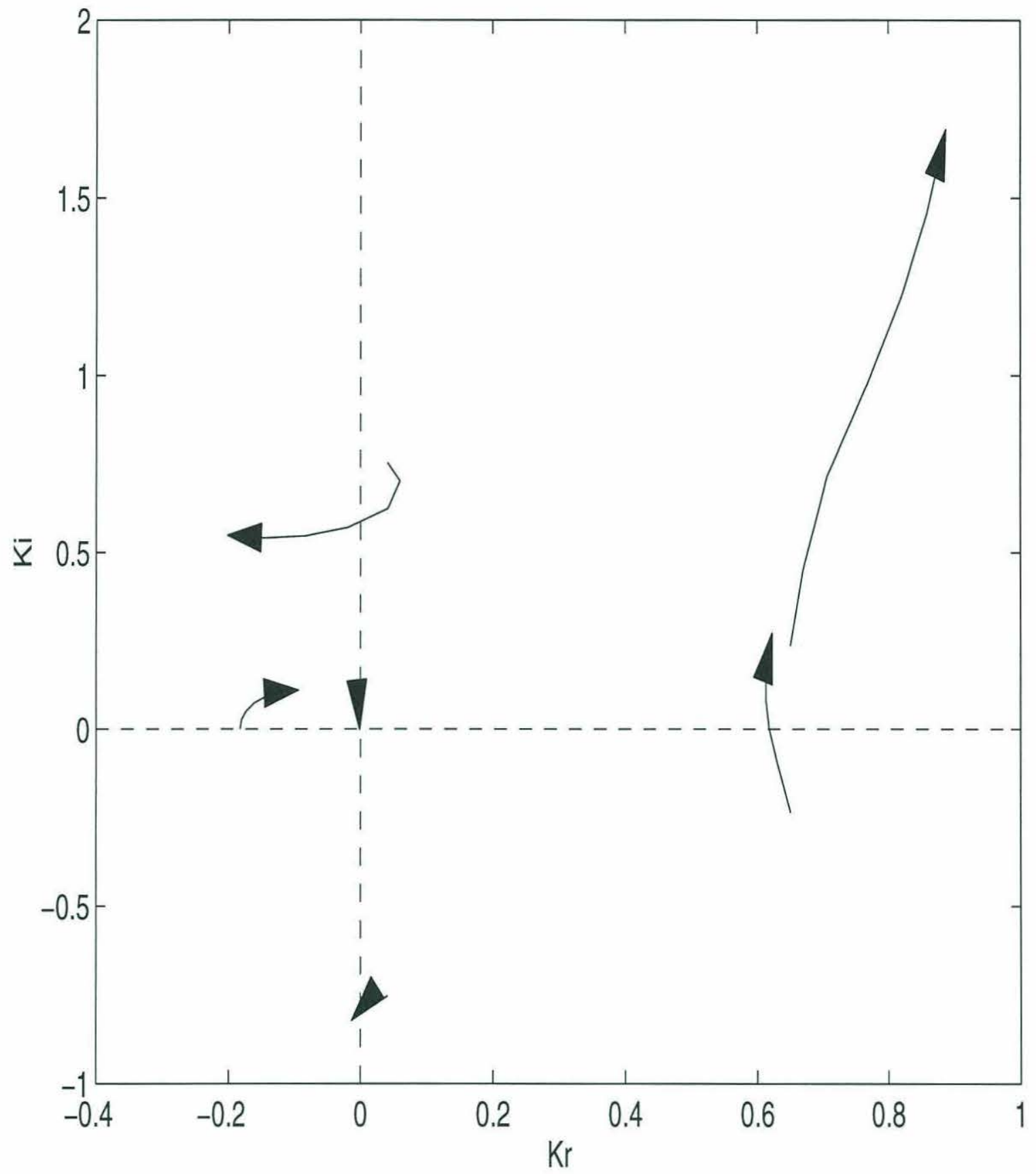

Figure 2.5: Track of $k_{i}$ as $\omega_{i}$ increases 
where $A_{0}$ is the initial amplitude of the wave. Following an individual peak, the phase factor $k_{r}(x-c t)$ is constant, which leads to constant $-k_{i}(x-c t)$ and hence constant $A_{1}$. So the wave propagates (except when $c=0$ ) but does not grow in time and space, or more explicitly its decay in time $-\omega_{i} * t=k_{i} c t$ and growth in space $-k_{i} x$ exactly offset each other. Essentially each peak proceeds downstream without changing amplitude, but successive peaks generated at the source become weaker and weaker with time. Thus a pattern with small amplitude waves near the source and larger amplitudes downstream is set up, giving the false appearance of a spatial instability.

When $c=0$, the wave does not propagate and does not grow in time eitherthis corresponds to a steady, spatially growing pattern. Since the wave does not propagate, the pattern cannot be set up by a localized forcing. Without applying Briggs' criterion directly to his problem, I cannot rule out the possibility that the steady, spatially growing pattern is a true spatially growing wave. However, it is most likely that $c=0$ corresponds to a neutral wave as well because neighboring positive and negative real $c$ correspond to neutral waves. Figure 2.4 shows that the Phillips' model example does not have spatially unstable modes at $\omega=0$.

\subsubsection{Pulse Instability}

Temporal instability assumes that disturbances are periodic in the horizontal direction. This is not proper when the initial disturbance is localized in a region. In this case it is more appropriate to solve the initial value problem. An initial value problem usually involves the use of a Fourier transform in space and a Laplace transform in time. Thus the double inversion of the transforms must finally be done to convert solution back to physical space and time. This is generally a formidable task even for the simplest models. However, the asymptotic form of the solution can be obtained for large times by using a Greens function method (Briggs, 1964). 
Briggs shows that the asymptotic response to a localized forcing or a pulse comes from the double roots of the dispersion relation enclosed in the deformed Fourier contour. However, the only contributions to the asymptotics come from double roots which are formed by the coalescence of two $k$ roots originating from opposite sides of the real $k$ axis. The roots move together as we shift the Laplace inversion contour. He also shows that a double root is equivalent to a saddle point. Hence the asymptotic approach with a Greens function used by Briggs is equivalent to a saddle point approach given that each saddle point found has been formed by two roots of $k$ originating from the opposite sides of real $k$ axis (Briggs absolute instability criterion). Here absolute instability occurs in a frame moving at a given group velocity, rather than a fixed point, except when the group velocity is zero. The criterion is crucial for a saddle point approach to give a correct solution, as emphasized by Pierrehumbert (1986). Interested readers are referred to Briggs (1964) and Pierrehumbert (1986).

The pinch singularity method Farrell (1982) will be used to find saddle points for given group velocity. It is the extension to the complex plane of the stationary phase technique for stable wave packets. I will follow Farrell's argument (1982) to introduce the pinch singularity method, but interpret it in a slightly different but equivalent way - as a version of the steepest descent method.

The solution of the initial value problem can be expressed as an integral over the normal mode solutions consisting of the exponentially growing and decaying modes plus another integral over a continuous spectrum. The decaying modes do not contribute for a large time. The contribution of the continuous spectrum is at most $\mathrm{O}(\mathrm{t})$ (Burger, 1966), and is negligible comparing to the exponentially growing modes for a sufficient large time. The asymptotic solution then can be expressed as an integral over the exponentially growing modes only,

$$
\psi(x, z, t)=\int_{-\infty}^{\infty} d k a(k) \psi_{k}(z) e^{i(k x-\omega t)}
$$


where $a(k)$ is the projection of initial conditions on the normal mode at $k$ and $\psi_{k}(z)$ the vertical structure of the mode at $k$. In order to look at asymptotic behavior for $t \rightarrow \infty$ but fixed $\frac{x}{t}$, we define

$$
\Omega(k)=\omega(k)-k \frac{x}{t}
$$

In the Equation (2.3.3), variable $k$ is real and runs from $-\infty$ to $+\infty$. However, according to the Cauchy theorem, if the integrand is an analytical function of $k$, we can deform the integrating contour arbitrarily without changing the integral. It is reasonable to assume analyticity of $a(k) . \Omega(k)$ or $\omega(k)$ may have branch points, but we assume we can avoid going around these points (I will return to this issue shortly in this subsection). Then we can deform the integrating contour to take a steepest descent path through the saddle point (see, e.g., Bender and Orszag, 1978). This implies $\Omega_{i}(k)=\omega_{i}(k)-k_{i} \frac{x}{t}$ will be constant along the path, i.e.

$$
\left.\frac{\partial}{\partial k} \Omega\right|_{k_{s}}=0
$$

And in the vicinity of the saddle point

$$
\Omega \simeq \Omega\left(k_{s}\right)+\frac{1}{2} \Omega^{\prime \prime}\left(k_{s}\right)\left(k-k_{s}\right)^{2}
$$

where

$$
\Omega^{\prime \prime}\left(k_{s}\right)=\frac{\partial^{2}}{\partial k^{2}} \Omega\left(k_{s}\right)
$$

Choose the path so that $-\frac{i}{2} \Omega^{\prime \prime}\left(k_{s}\right)\left(k-k_{s}\right)^{2}$ is real and negative, so that we have

$$
\psi(x, z, t) \cong a\left(k_{s}\right) \psi_{k_{s}}(z) e^{-i \Omega\left(k_{s}\right) t} I\left(k_{s}, t\right)
$$

where

$$
\begin{aligned}
I\left(k_{s}, t\right) & =\int_{-\infty}^{\infty} d k e^{-\frac{i}{2} \Omega^{\prime \prime}\left(k_{s}\right)\left(k-k_{s}\right)^{2} t} \\
& =\frac{1}{\sqrt{\pi\left|\Omega^{\prime \prime}\left(k_{s}\right)\right| t / 2}}
\end{aligned}
$$


And finally we get

$$
\begin{array}{r}
\psi(x, z, t)=b\left(k_{s}\right) \psi_{k_{s}}(z) t^{-1 / 2} e^{-i \Omega\left(k_{s}\right) t} \\
=b\left(k_{s}\right) \psi_{k_{s}}(z) t^{-1 / 2} e^{i\left(k_{s} x-\omega\left(k_{s}\right) t\right)}
\end{array}
$$

If $\Omega_{i}>0$, the system is unstable, and the growth rate is $\Omega_{i}$ at the point. For a pair of $x$ and $t$ given, if there is such a point, the growth rate will be valid for any pair of $x$ and $t$ as long as $\frac{x}{t}$ is the same. This is to say that an observer in a reference frame moving at $\frac{x}{t}$ will see the disturbance growing at the same growth rate $\Omega_{i}\left(k_{s}\right)$. Equation (2.3.7) gives the asymptotic solution seen by the observer.

From Equation (2.3.6), we see that the asymptotic solution may break down when $\Omega^{\prime \prime}\left(k_{s}\right)$ is close to zero, i.e., the group velocity is close to its maximum or minimum points. In that case, the expansion in Equation (2.3.5) has to be carried to the third order.

It is easy to see according to Cauchy-Riemann condition that $\frac{\partial}{\partial k} \Omega=0$ leads $\frac{\partial}{\partial k_{r}} \Omega=0$, and hence

$$
\frac{\partial}{\partial k_{r}} \Omega_{r}+i \frac{\partial}{\partial k_{r}} \Omega_{i}=\left(\frac{\partial}{\partial k_{r}} \omega_{r}-\frac{x}{t}\right)+i \frac{\partial}{\partial k_{r}} \omega_{i}=0
$$

By definition, we have $C_{g}=\frac{\partial}{\partial k_{r}} \omega_{r}$ and hence at the saddle point $k=k_{s}, C_{g}=\frac{x}{t}$ for given $x$ and $t$. In other words, given $x$ and $t$, the wave which dominates the point $x$ at the time $t$ has a group velocity $C_{g}=\frac{x}{t}$. If we can find solutions for all $C_{g}$, we have solutions for any $x$ and $t$ given large $t$. It is important to remember that $C_{g}$ is evaluated at a complex value of $k$, not along the real axis.

In the above derivation, I have assumed that when $\Omega(k)$ or $\omega(k)$ have branch points, we can avoid going around these points in some way. It is difficult to prove this assumption mathematically. In some cases, (Pierrehumbert, 1986), the contour cannot be deformed to the saddle point and saddle point analysis gives incorrect results. For our full model in later chapters, the solutions are too complex for a 
detailed analysis of how the contour can be moved. Instead, we show that direct numerical simulation of pulse instability agrees well with the saddle point analysis. Readers are referred to page 72 and Figure 2.26 in Section 2.5 for such a comparison.

The pinch singularity method, as long as all saddle points satisfy Briggs' absolute instability criterion, can be used to determine whether a system is absolutely or convectively unstable. If such a saddle point is found for $C_{g}=\frac{x}{t}=0$ with $\Omega_{i}\left(k_{s}\right)>0$, the system is absolutely unstable. The amplitude of wave envelope at $x=0$ (the point of excitation) will grow as $\exp \left(\Omega_{i}\left(k_{s}\right) t\right)$. And for any $x$ when $t$ is large, the amplitude of the local envelope grows at the rate

$$
\nu_{i}=\omega_{i}\left(k_{s}\right)-\operatorname{Im}\left(k_{s}\right) \frac{x}{t}
$$

This is a simpler method to determine whether a system is absolutely or convectively unstable in comparison with the Greens Function method used by Merkine (1977) and Thacker (1977). Although the latter can provide an analytical criterion for a simple two-layer model, its application to an even slightly more realistic model will be extremely tedious and complicated. The steepest descent method only provides an asymptotic solution for large $t$, while the Greens Function method can provides a full solution as long as the integral can be done. However the difficulty in carrying out the integral generally limits its power to such a extent that eventually an asymptotic approach has to be taken.

Figure 2.6 shows the results of pulse instability. Growth rate is shown in the bottom panel, frequency and wavenumber in the middle and upper panel respectively. The ' $\mathrm{x}$ ' marks indicate values corresponding to the maximum of the envelope growth, which is the same as the temporally most unstable wave. This is always true because $\frac{\partial}{\partial k_{r}} \omega_{i}=0$ for the most unstable wave, which means that in the plane of $\omega$ as a function of $k$, that point itself is a saddle point for a reference frame moving at $C_{g m} . C_{g m}$ is the group velocity corresponding to the temporally most unstable wave. This information gives a starting point to search for other saddle points incrementally away from $C_{g m}$. 

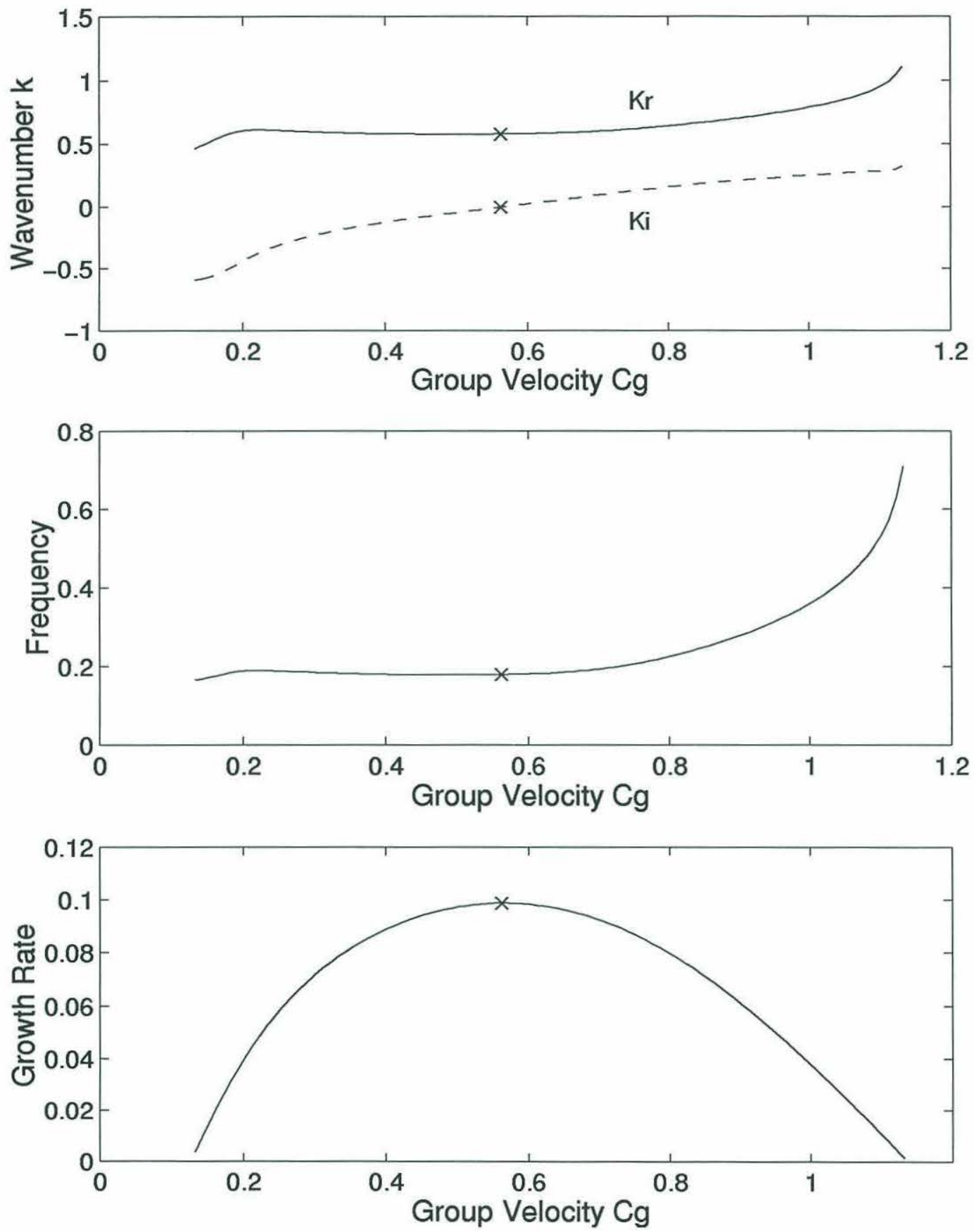

Figure 2.6: Pulse instability. 
Since for different $C_{g}^{\prime} s$, real wave numbers are significantly different, pulse instability predicts variable space scales of a developing disturbance, while temporal instability assumes the same space scale everywhere.

\subsubsection{Comparison with Farrell's Model}

Farrell (1982) also studied pulse instability in a two-layer model with parameters tuned to atmospheric situation. The model dynamics are the same in his model and model studied here. He used $U_{1}=2, U_{2}=0, \beta=1$ and $F_{1}=F_{2}=1$. His results are shown (dashed curve) in Figure 2.7. I have rescaled his results so that they are comparable to mine. His $\beta=1$ is equivalent to $\beta=0.25$ in my model. The solid curves are the results we seen in the last subsection.

The lower panel shows the growth rates of the envelope. Although in his model, unstable waves extend to larger range of $C_{g}$, there does not seem to be much qualitative difference. His model has nonzero growth rate at $C_{g}=0$, implying absolute instability, whereas my model is convectively unstable. This is simply due to choice of mean velocity. If we add a uniform barotropic velocity $U_{0}$ to the system, the growth rates will only shift by $U_{0}$ in the $C_{g}$ coordinate. However, our results are different from those of Farrell's (1982) in one aspect. From the upper panel of Figure 2.7, in the two-layer oceanic model used here, real wavenumber increases significantly when the group velocity increases while in Farrell's two-layer atmospheric model, the real wavenumber decreases when the group velocity increases.

This means that in the oceanic case, signals of short waves travel faster than long waves, while the opposite is true in the Farrell's model. Hence the structures of growing wave packets in the two models will be qualitatively different. This implies we cannot generalize to the oceanic model the conclusion Farrell made regarding cyclogenesis in the atmosphere that slowly traveling short waves contribute more to 
absolute instability if there is any. For the parameters chosen above, the system is convectively unstable since $\nu_{i}$ is zero for $C_{g}=0$. And if we reduced the barotropic component of the flow, we would find absolute instability with longer waves growing.

In order to investigate why such a difference exists, I will vary two independent variables $\delta=H_{1} / H_{2}$ and $\beta$ with velocity shear $U_{1}-U_{2}=1$ fixed. Here $\beta$, the nondimensional gradient of planetary vorticity, is rescaled as $\beta=\frac{\beta_{0} L^{2}}{\left(U_{1}-U_{2}\right)}$ (see Equation (2.2.3a)).

In the two-layer model, with $U_{1}-U_{2}=1$, the critical value of $\beta$ for the system to be unstable is $F_{2}=\frac{\delta}{(1+\delta)}$. In the range of $\left[\begin{array}{ll}0 & F_{2}\end{array}\right]$, I consider three different cases; the first, $\beta=0.1 * F_{2}$; the second, $\beta=0.5 * F_{2}$, which is the midpoint of the range; and the third, $\beta=0.9 * F_{2}$, which is close the critical value. These three cases are shown in the upper, middle and lower panels of Figure 2.8 respectively. Only the real part of wavenumber is drawn as a function of group velocity for each pair of $(\delta, \beta)$. The value of $\delta$ is labeled in the figure near its corresponding curve.

From the upper panel of Figure 2.8, we see that when $\beta$ is small, the real part of wavenumber is increasing in the forward segment of the wave packet except in the very leading edge. The slope depends on the value of $\delta$, but the upsloping trend does not. In the backward segment, the trend is sensitive to the value of $\delta$ except in the very trailing edge: fairly flat or upsloping when $\delta=0.2$, and downsloping for the other cases $\left(\delta=0.6\right.$ or 1.0). In the middle panel, when $\beta=0.5 * F_{2}$, the trend in the backward segment is similar to the upper panel. However, the trend in the forward part is very sensitive to the value of $\delta$. For small $\delta$, the curve is upsloping whereas for large $\beta$ it is downsloping. The transition happens between 0.5 and $0.6 . \delta=1$ case is the case Farrell studied and $\delta=0.2$ is close to the case I shown in the previous subsections. From the lower panel, when $\beta$ is close to its critical value, the trend is similar for all values of $\delta$. 

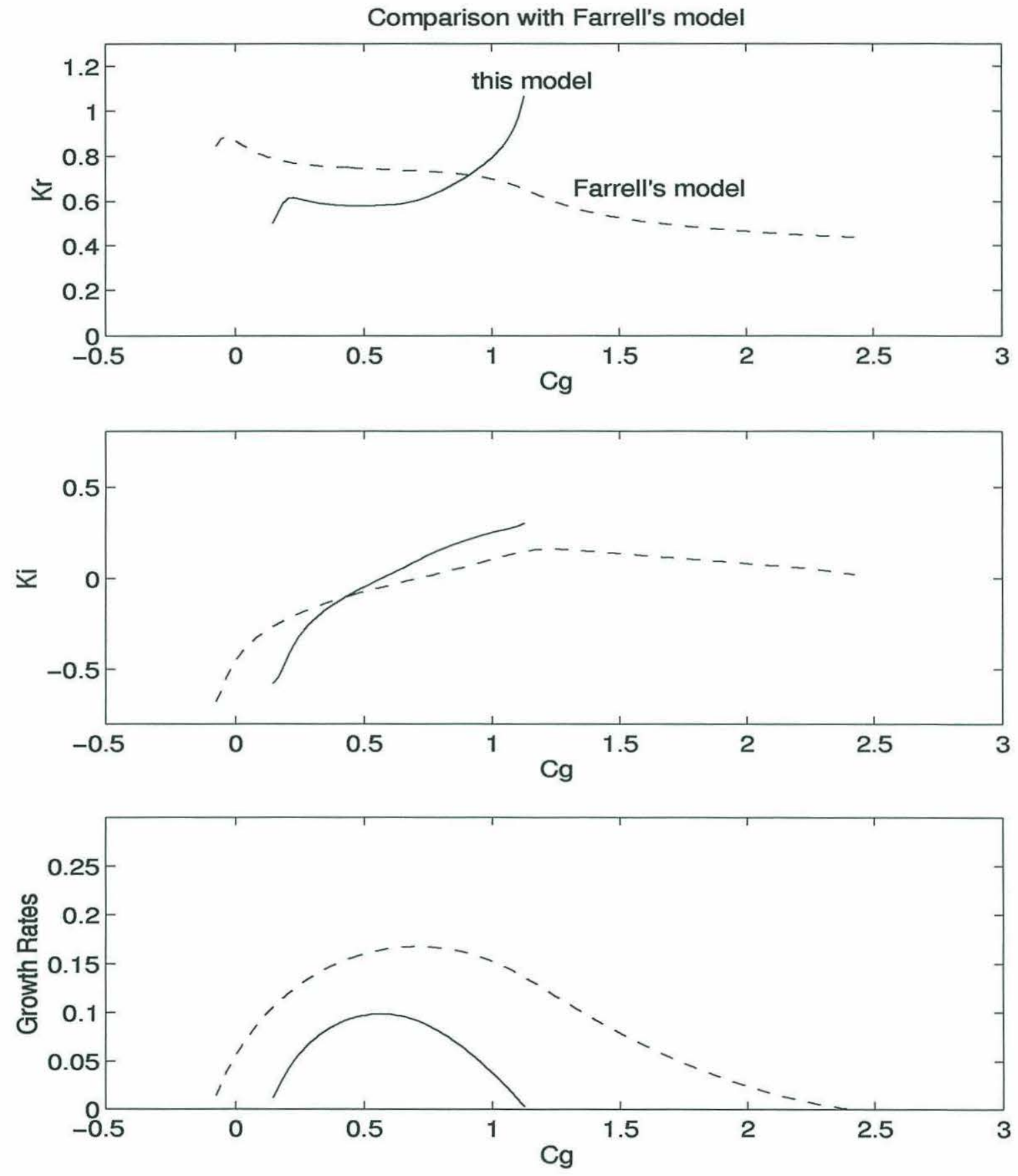

Figure 2.7: Comparison with Farrell's Model. Solid curves indicate the results from my model. $U_{1}=1, U_{2}=0.2, \delta=0.2$ and $\beta=0.05$. Dashed curves show Farrell's results rescaled to be comparable. $U_{1}=1, U_{2}=0, \delta=1$ and $\beta=0.25$. 
Beta $=0.1^{*} \mathrm{~F} 2$
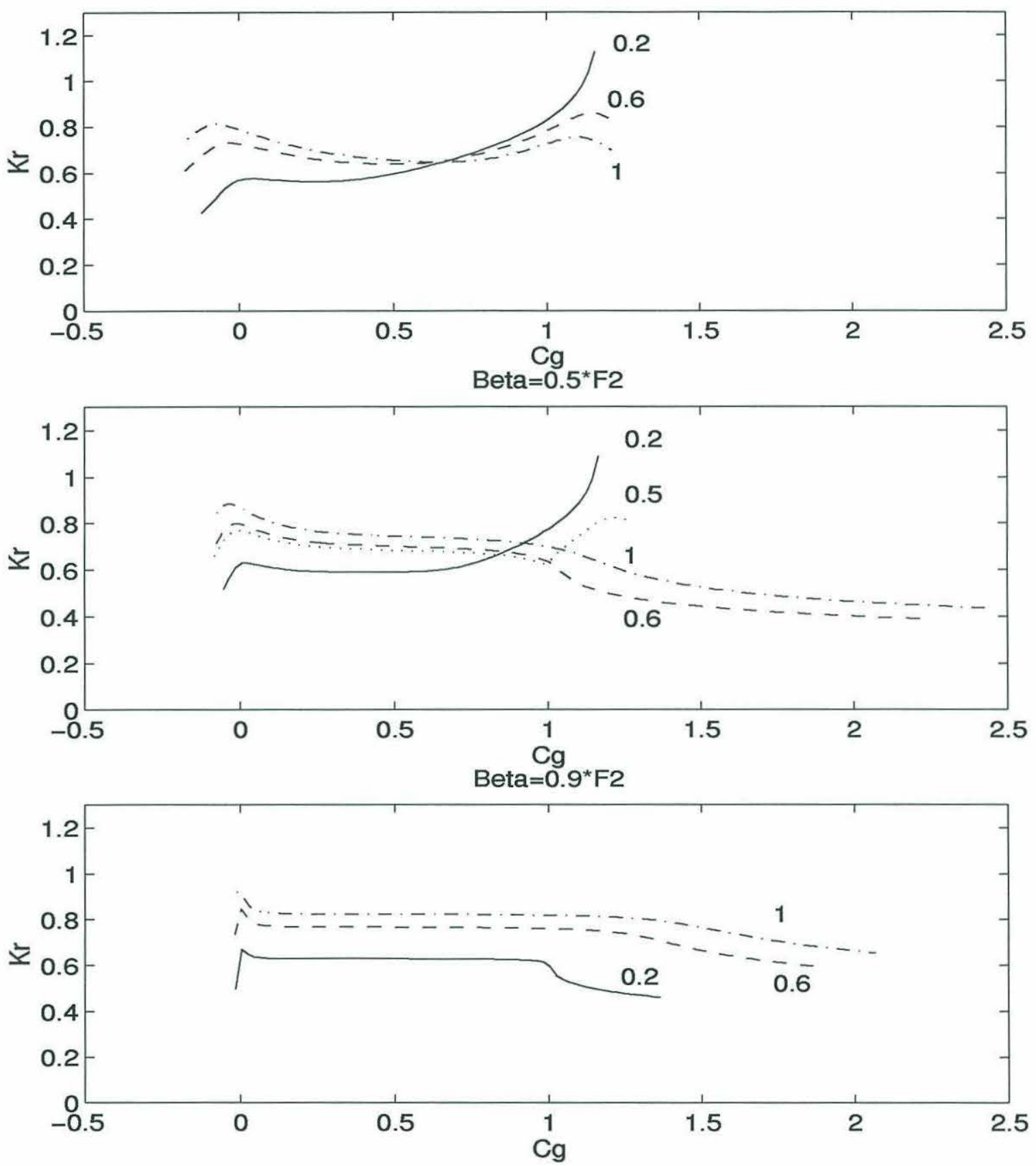

Figure 2.8: Real part of wavenumber as a function of group velocity. The value of $\beta$ used is indicated at the top of each panel. The value of $\delta$ is labeled near its corresponding curve. 
In summary, for the oceanic case where $\delta$ is small, short waves tend to appear in forward segment of the wave packet except when $\beta$ is very close to its critical value. For the atmospheric case where $\delta$ is large, long waves tend to appear in forward segment of the wave packet except when $\beta$ is close to zero. According to Stone's argument of baroclinic adjustment (1978), $\beta$ in the atmosphere is close to critical value, so the trend in Farrell's model seems reasonable in atmosphere, although the value of $\beta$ he used is not close to critical value. The Gulf Stream, on the other hand, is very far from critical. 


\subsubsection{The Growth Rate of an Individual Peak in Pulse In- stability}

In the previous section, we have seen that when a disturbance is generated in a local region in an unstable system, the disturbance will evolve into its asymptotic form after a large time. The asymptotic form (Equation (2.3.7)) is

$$
\begin{array}{r}
\psi(x, z, t)=b\left(k_{s}\right) \psi_{k_{s}}(z) t^{-1 / 2} e^{-i \Omega\left(k_{s}\right) t} \\
=b\left(k_{s}\right) \psi_{k_{s}}(z) t^{-1 / 2} e^{i\left(k_{s} x-\omega\left(k_{s}\right) t\right)}
\end{array}
$$

Also we have shown that given $x$ and $t$, the wave which dominates the point $x$ at the time $t$ has a group velocity $C_{g}=\frac{x}{t}$. The growth rate of the amplitude of the local envelope, for any $x$ when $t$ is large, is given by Equation (2.3.8), repeated below for convenience.

$$
\nu_{i}=\omega_{i}\left(k_{s}\right)-\operatorname{Im}\left(k_{s}\right) \frac{x}{t}
$$

One interesting feature of the pulse instability is that the individual peaks generally grow at rates different from that of the envelope (Simmons and Hoskins, 1979). This is because an individual peak propagates at phase speed which is different from the group velocity. It is easier to understand this phenomenon in a neutral wave packet. For a neutral wave packet, the envelope decays at a rate proportional to $t^{-1 / 2}$ due to dispersion. For the simplicity of demonstration, we assume the envelope does not decay. Even in this case, if an individual peak at the forward paradigm of the envelope propagates at a phase speed less than the group velocity, it actually moves towards the center of the wave packet. Hence its amplitude is increasing, and so its growth rate is positive. On the other hand, if an individual peak at the trailing edge propagates at a phase speed less than the group velocity, it actually moves away from the center of the wave packet. Hence its amplitude is decreasing, and so its growth rate is negative. Therefore, even if the envelope itself does not grow or decay, an 
individual peak can still grow or decay depending on its relative position in the wave packet.

In the case that the wave envelope also grows, the feature is still the same because the amplitude of the envelope is positively and exponentially related to the growth rate at the location. In fact, the differential in the envelope growth enhances such a feature. When an individual peak in the forward paradigm of the envelope propagates at a phase speed less than the group velocity, it not only moves toward a region with a larger amplitude and but also toward a region with a larger local growth rate (the growth rate of the envelope at the location). Therefore it will appear to grow at a rate higher than the local growth rate in addition to the amplitude differential effect. The reasoning can be similarly applied to the case when a peak moves toward a region with a lower growth rate and a smaller amplitude. The opposite is true in this case.

Because the amplitude of a peak is equal to the amplitude of the envelope at the same location, we can calculate the growth rate of the peak from the growth rate of the envelope. Suppose the disturbance is generated at or around $x_{0}=0$ and at $t_{0}=0$. After a large time $t$, we start to keep a track of an individual peak in the well developed wave envelope. The positions of the peak at $t$ and $t+\delta t$ are $x$ and $x+\delta x$ respectively (see Figure 2.9), and the corresponding group velocities $C_{g}$ and $C_{g}+\delta C_{g}$. The average phase speed of the peak between $t$ and $t+\delta t$ is $C_{p h}$. Consider $\delta t \rightarrow 0$

$$
\begin{aligned}
C_{p h} & =\lim _{\delta t \rightarrow 0} \frac{\delta x}{\delta t} \equiv \dot{x} \\
C_{g} & =\frac{x}{t}
\end{aligned}
$$

and the amplitude of the peak at $t$ is equal to the amplitude of the envelope at $x$, which is 


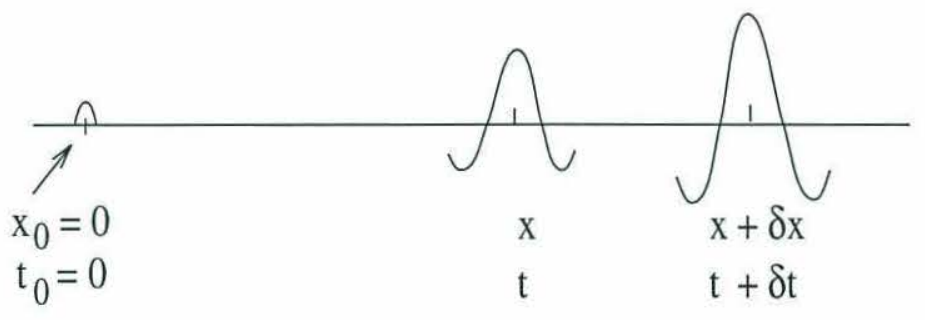

Figure 2.9: Schematic picture of an individual peak moving downstream.

$$
\begin{aligned}
A & =\left|b\left(k_{s}\right) \psi_{k_{s}}(z)\right| t^{-1 / 2} e^{\nu_{\mathrm{i}}\left(C_{g}\right) t} \\
& =\alpha\left(C_{g}\right) t^{-1 / 2} e^{\nu_{\mathbf{i}}\left(C_{g}\right) t}
\end{aligned}
$$

where $\alpha\left(C_{g}\right) \equiv\left|b\left(k_{s}\right) \psi_{k_{s}}(z)\right|$. Note that $k_{s}$ only depends on $C_{g}$. $\alpha$ also depends on $z$ but this dependence is not essential in the derivation and it is omitted for convenience.

The growth rate of the peak is

$$
\begin{aligned}
\nu_{\text {ipeak }} & =\frac{1}{A} \frac{d A}{d t}=\frac{d \ln (A)}{d t} \\
& =\frac{\partial \ln \alpha}{\partial C_{g}} \dot{C}_{g}-\frac{1}{2 t}+\nu_{i}+\frac{\partial \nu_{i}}{\partial C_{g}} \dot{C}_{g} t
\end{aligned}
$$

where a dot over a variable means the first order derivative of the variable with respect to $t$.

$$
\begin{aligned}
\dot{C}_{g} & \equiv \frac{d}{d t} C_{g} \equiv \frac{d}{d t} \frac{x}{t} \\
& =\left(-C_{g}+\dot{x}\right) / t=\left(C_{p h}-C_{g}\right) / t
\end{aligned}
$$

these relations lead to

$$
\nu_{\text {ipeak }}=\left(\frac{\partial \ln \alpha}{\partial C_{g}}\right)\left(C_{p h}-C_{g}\right) / t-\frac{1}{2 t}+\nu_{i}+\left(C_{p h}-C_{g}\right) \frac{\partial \nu_{i}}{\partial C_{g}}
$$




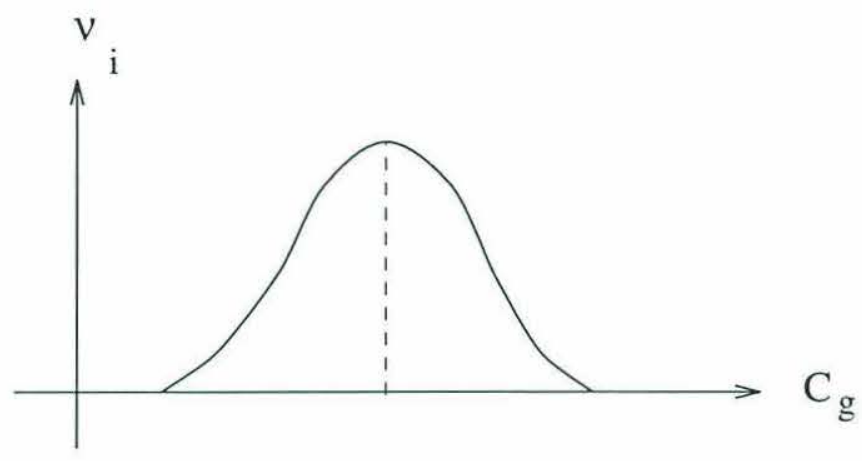

Figure 2.10: An example of the growth rate of the envelope as a function of $C_{g}$.

The first two terms in Equation (2.3.12) are proportional to $1 / t$. Hence for large $t$, we have ${ }^{1}$

$$
\nu_{i p e a k}=\nu_{i}\left(C_{g}\right)+\left(C_{p h}-C_{g}\right) \frac{\partial \nu_{i}}{\partial C_{g}}
$$

Given the growth rate of the envelope in Figure 2.10 and $C_{g}>C_{p h}$, individual peaks in the leading edge grow faster than the maximum growth of the envelope, while peaks in the trailing edge grow slower than the maximum growth of the envelope. This relation was first found by Simmons and Hoskins (1979) in a numerical experiment on unstable baroclinic waves in atmosphere. Equation (2.3.13) was also given in their paper without a mathematical derivation. Here I have provided a detailed theoretical derivation.

In Section 2.5, I will demonstrate in detail how to calculate the growth rates of the envelope and individual peaks from numerical experiments.

Note that we can similarly derive a more accurate formula for the growth rate of the envelope $\left(\nu_{i_{\text {adj }}}\right)$. In fact, we can start with right hand side of Equation (2.3.10), and use the relations $\dot{C}_{g} \equiv 0$ for the envelope, to get

$$
\nu_{i_{-} a d j}=-\frac{1}{2 t}+\nu_{i}
$$

\footnotetext{
${ }^{1}$ In the case of a neutral packet, $\nu_{i}=0$, the first two terms are all that appear and we see that a peak can still grow, if it moves from the front of a packet towards the maximum of the envelope sufficiently rapidly to overcome the spreading of the packet. But the growth rate decreases as $\frac{1}{t}$.
} 
the correction term $-\frac{1}{2 t}$ may be significant when $t$ is small. The $O(1 / t)$ term will be detectable in the numerical experiments in Section 2.5. And in Chapter 5, we will see that it takes much less time for a wave packet to reach $\nu_{i_{-} \text {adj }}$ than $\nu_{i}$. 
Given the growth rate of the envelope $\nu_{i}$ in Figure 2.6, we can calculate the growth rate of an individual peak $\nu_{\text {ipeak }}$ from Equation (2.3.13). Figure 2.11 shows the comparison. Figure 2.11 is the same as Figure 2.6 except that the dark solid curve in the lower panel indicates growth rate of an individual peaks. It has a maximum at $C_{g}=1.02$; this point and corresponding frequency and wavenumber are marked with dark 'x's. The maximum growth (0.176) exceeds the maximum growth of the envelope (0.099) by $78 \%$. The corresponding frequency and wavenumber are also higher than their counterparts (marked by 'x's) associated with the maximum of the envelope. The $\nu_{\text {ipeak }}$ curve crosses the maximum of $\nu_{i}$ curve since at that point $\frac{\partial \nu_{i}}{\partial C_{g}}=0$. Behind the maximum of the envelope, an individual peak grows slower than the envelope. $\nu_{i p e a k}$ curve crosses $\nu_{i}$ curve once more at $C_{g}=0.32$ due to zero $C_{p h}-C_{g}$. A peak behind the crossing point can also grow faster than its envelope. This feature that is near the trailing edge the individual peaks grow faster than the envelope does not appear clearly in the QG and shallow-water Gulf Stream models in Chapter 3 and 4 (see Sec. 3.5. and 4.3.), possibly due to the jet structure used there.

Given the growth rate of individual peak $\nu_{\text {ipeak }}$, if we focus on a peak in the leading edge of a well developed disturbance envelope, we can predict its future position and amplitude by integration. Assume a starting amplitude of 1 for simplicity. Given $C_{g}=x / t$, we can look up $\nu_{i p e a k}$ and $C_{p h}$ for that spot, from which we compute the position and amplitude of the peak for next time step. Repeating the procedure step by step, we collect the history of the individual peak. Figure 2.12 shows the results of such an experiment. The integration starts at $C_{g}=1$. In the figure, growth rate, phase speed and wavenumber are plotted vs time. We see that the growth rate, phase speed and wavenumber of the individual peak decrease as the peak evolves. When the peak is on the leading edge of the envelope, its growth rate can be much larger than that of maximum envelope growth. 

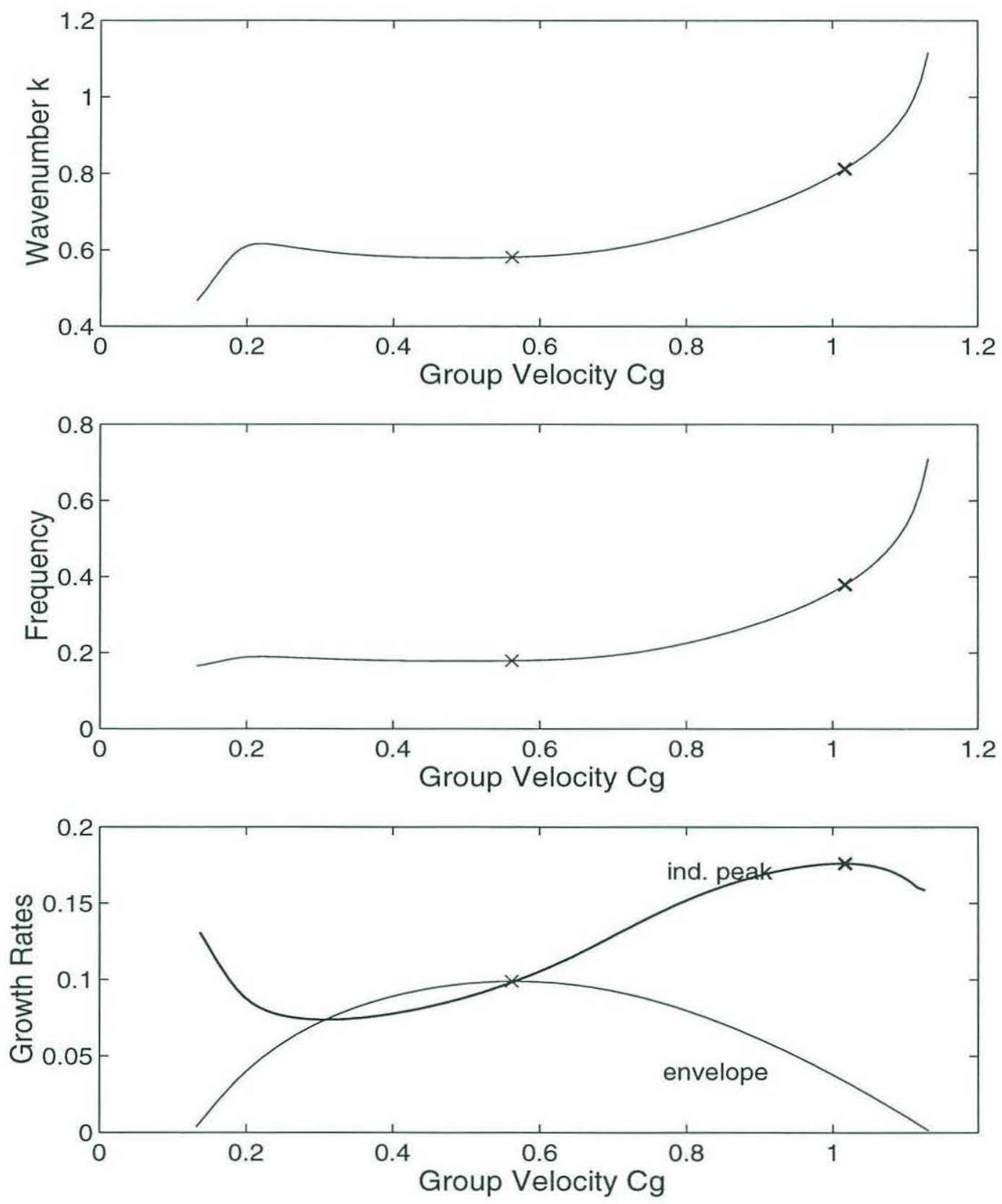

Figure 2.11: Comparison between the growth rates of the envelope and an individual peak. 
Alternatively, we can plot growth rate, phase speed and wavenumber as functions of amplitude or logarithm of amplitude. We scale the amplitude of the peak by its starting amplitude. Figure 2.13 shows these quantities vs logarithm of amplitude. The pattern is very similar to Figure 2.12. The growth rate of the individual peak decreases as the amplitude increases, and so does the wavenumber. This seems like a nonlinear effect of amplitude on the growth rate, but, instead is an intrinsic property of pulse instability and has nothing to do with nonlinearity since we are using a linear model. Because the amplitude has been scaled by its starting amplitude, the absolute amplitude is not important here. It is not the amplitude itself that matters but the position of the peak relative to the maximum of the envelope. Given a dispersion relation and a starting amplitude of a peak in a well developed wave packet, the growth rate can be related to the subsequent amplitude, but the reduction of growth rate is not due to the increase of amplitude but rather to the shifting position within the packet.

The implication of this effect is that a set of peaks starting with the same amplitude but at different positions relative to the maximum of envelope will grow at different rates and have different properties.

This effect is an important difference of pulse instability from temporal and spatial instabilities. In a nonlinear system, this intrinsic property of pulse instability may be coupled with nonlinearity and it may become difficult to tell them apart. 

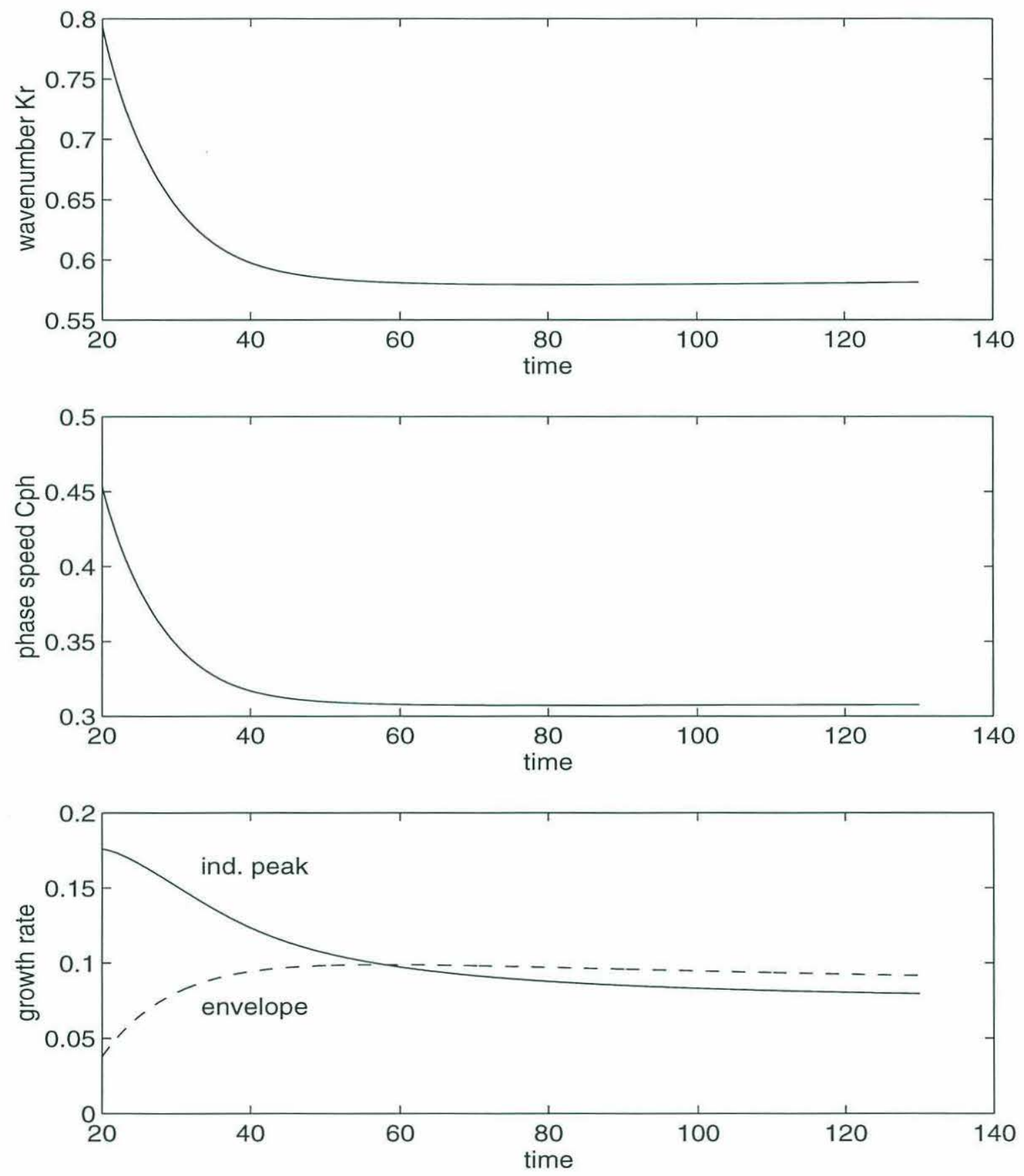

Figure 2.12: Properties of an individual peak vs time. $U_{1}=1$ and $U_{2}=0.2$ and $\beta=0.05$. The integration starts at $C_{g}=1$. 

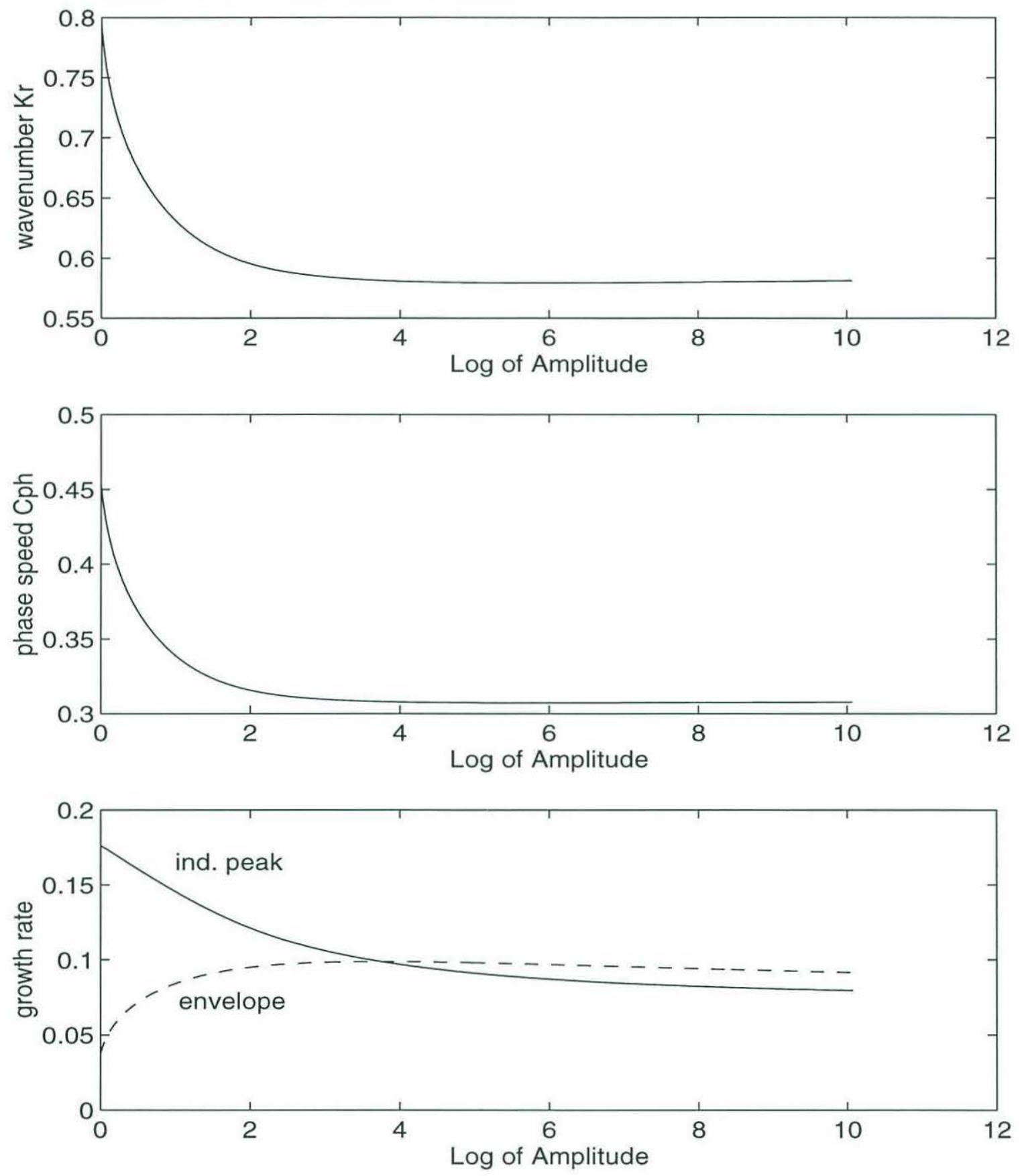

Figure 2.13: Properties of an individual peak vs log of amplitude. $U_{1}=1.0, U_{2}=0.2$ and $\beta=0.05$. 


\subsection{Comparison between the three types of insta- bility theories}

\section{Comparison between temporal and pulse instabilities}

In Figure 2.14, the lower panel compares the growth rates of temporal and pulse instabilities as functions of real part of wavenumbers. The growth rate here refers to the envelope growth in the case of pulse instability. The maximum growth rate and corresponding properties such as frequency and group velocity of the envelope in pulse instability is the same as the most temporally unstable wave as we have seen before. The corresponding properties (in upper panel) such as frequencies and group velocities in both theories are also the same for that wave.

One interesting feature shown in the figure is that pulse instability allows unstable waves to have wavenumbers beyond short wave cut-off of the temporal theory. This is reasonable because we are comparing the real part of the local wavenumber in pulse theory with a global wavenumber in temporal theory. The local wavenumber in pulse theory contains a nonzero imaginary part, so that even if its real part is the same as the wavenumber in temporal theory, its frequency $\omega\left(k_{r}+i k_{i}\right)$ has been extended to complex $k$ plane and is naturally different from that in temporal theory $\left(\omega\left(k_{r}\right)\right)$.

Around the most unstable wave, the growth rate and group velocity have multiple values corresponding to a wavenumber. $C_{g}$ in temporal theory is fairly uniform in the unstable range, while $C_{g}$ in pulse theory varies dramatically and can be different from the former by as much as $100 \%$. The frequencies in the two theories agree with each other reasonably well. The maximum difference occurs at the short wave cut-off of the temporal theory.

\section{Comparison between temporal and spatial instabilities}



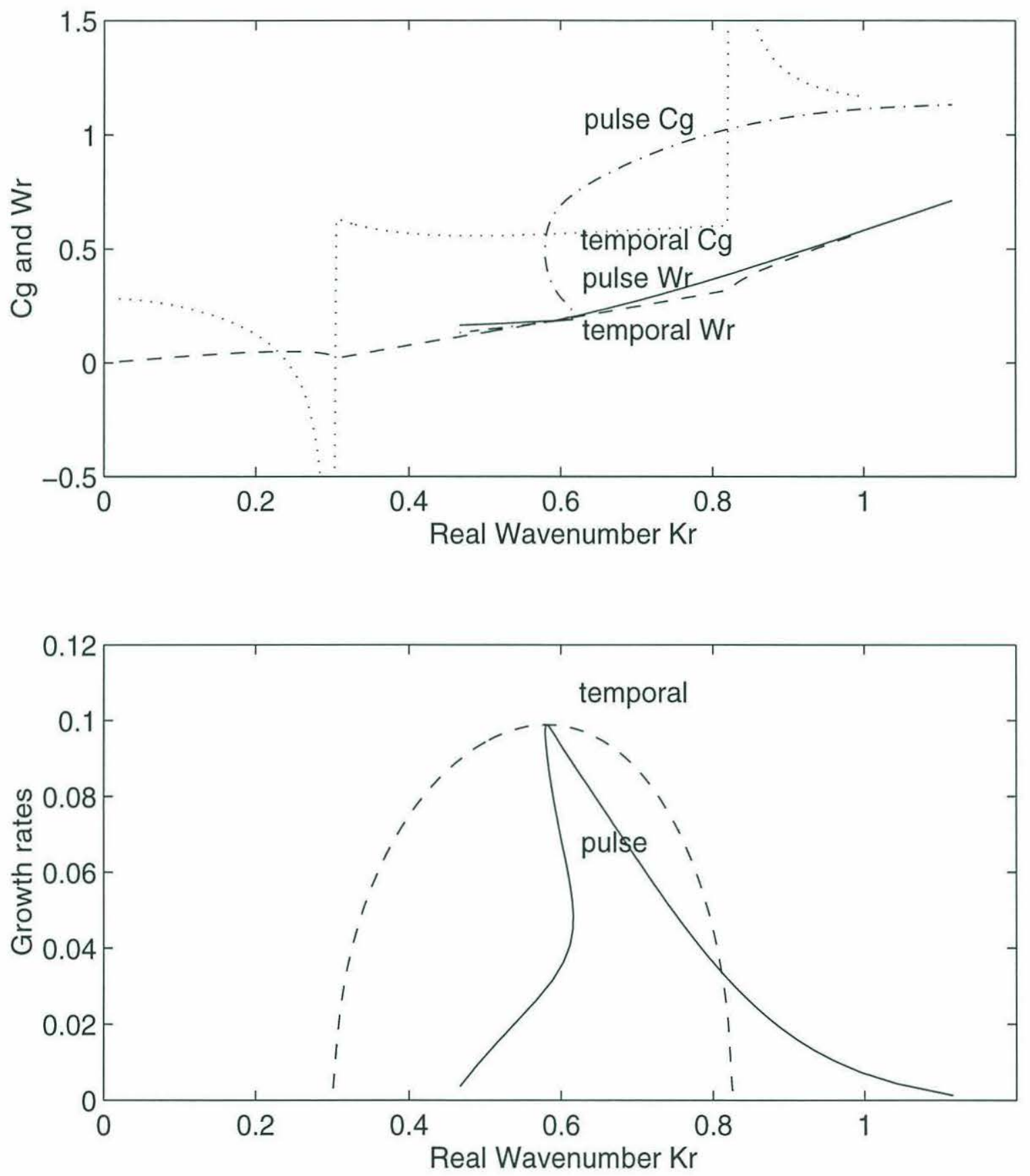

Figure 2.14: Comparison between temporal and pulse instabilities. 

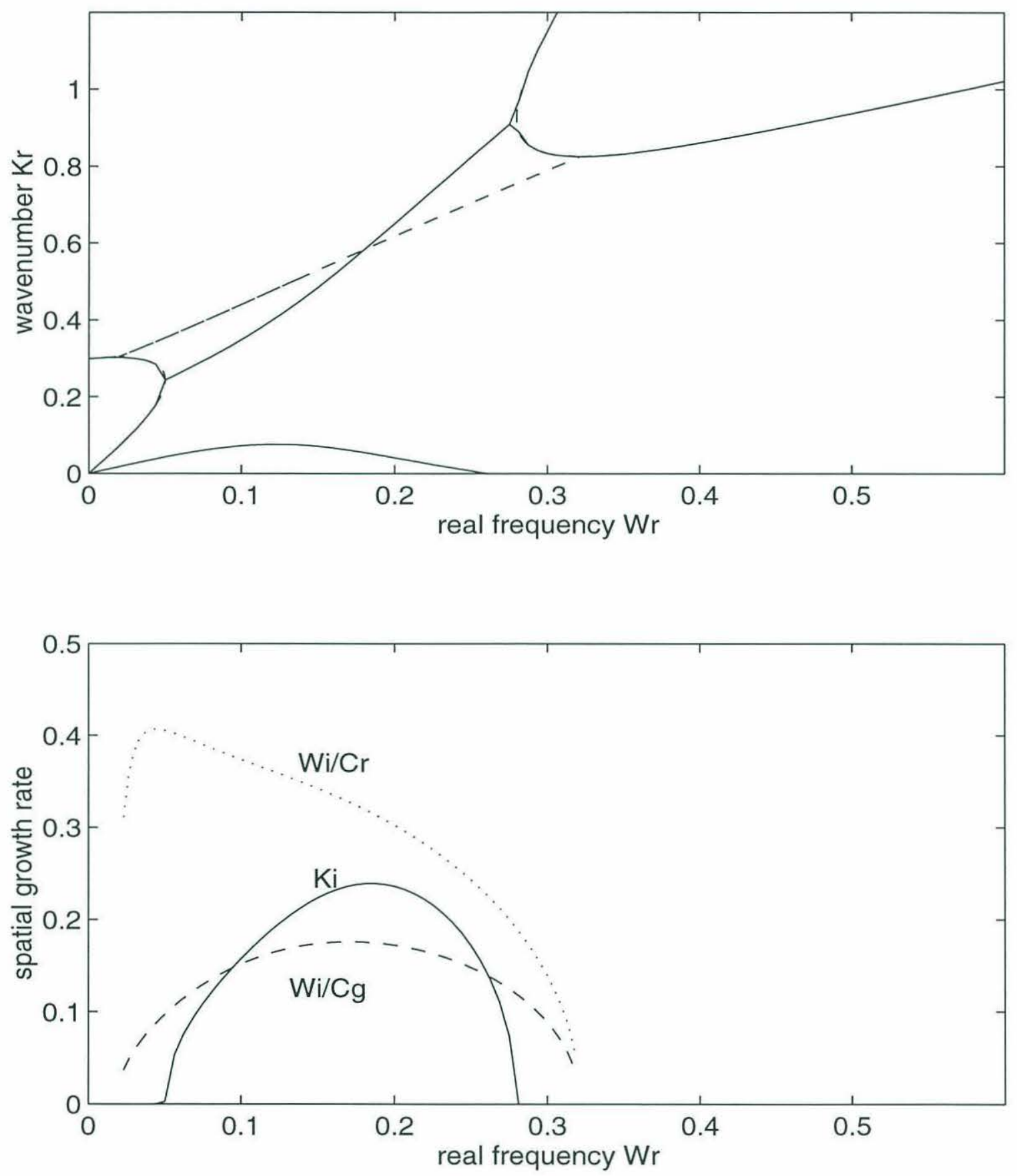

Figure 2.15: Temporal and Spatial, $U_{1}=1, U_{2}=0.2, \beta=0.05$ 
Figures 2.15 to 2.17 compare the differences between temporal and spatial instabilities. The solid line always represent the spatial growth rate (SGR) from spatial instability, while dashed lines the equivalent spatial growth rates(ESGR) estimated from the temporal growth rates divided by group velocity according to Gaster's transformation formula. The dotted line in the lower panels indicate another type of equivalent spatial growth rates transformed from the temporal rates divided by phase speed. Let us call it pseudo-equivalent spatial growth rate (PESGR). This transformation does not have the same theoretical support but has been used in some observational studies (e.g., Watts and Johns, 1982, Kontoyiannis, 1992). In the upper panels, the dashed lines represent the real wave number from temporal instability. The parameters are the same as before unless specified otherwise. I varied $\beta$ to change the growth rates.

In the lower panel of Figure 2.15, we see that for the most unstable waves, PESGR are larger and ESGR are smaller than SGR. Actually it is difficult to define the most unstable wave from PESGR because the small phase speed dominates the transformation. The SGR curve has narrower shape than ESGR. In the upper panel, we see that the wavenumbers of the most unstable waves from both theories are almost the same.

There seems no clear correlation between SGR and PESGR in Figure 2.15, because when the mean flow is slow, the transformation from temporal growth rate to PESGR is dominated by large variation of phase speed instead of that of growth rate itself. When the mean flow increases, relative differences among SGR,ESGR and PESGR decrease (comparing Figure 2.15 with 2.16).

Figure 2.17 shows a case where SGR is the largest among the three types of growth rates for the most unstable waves. 

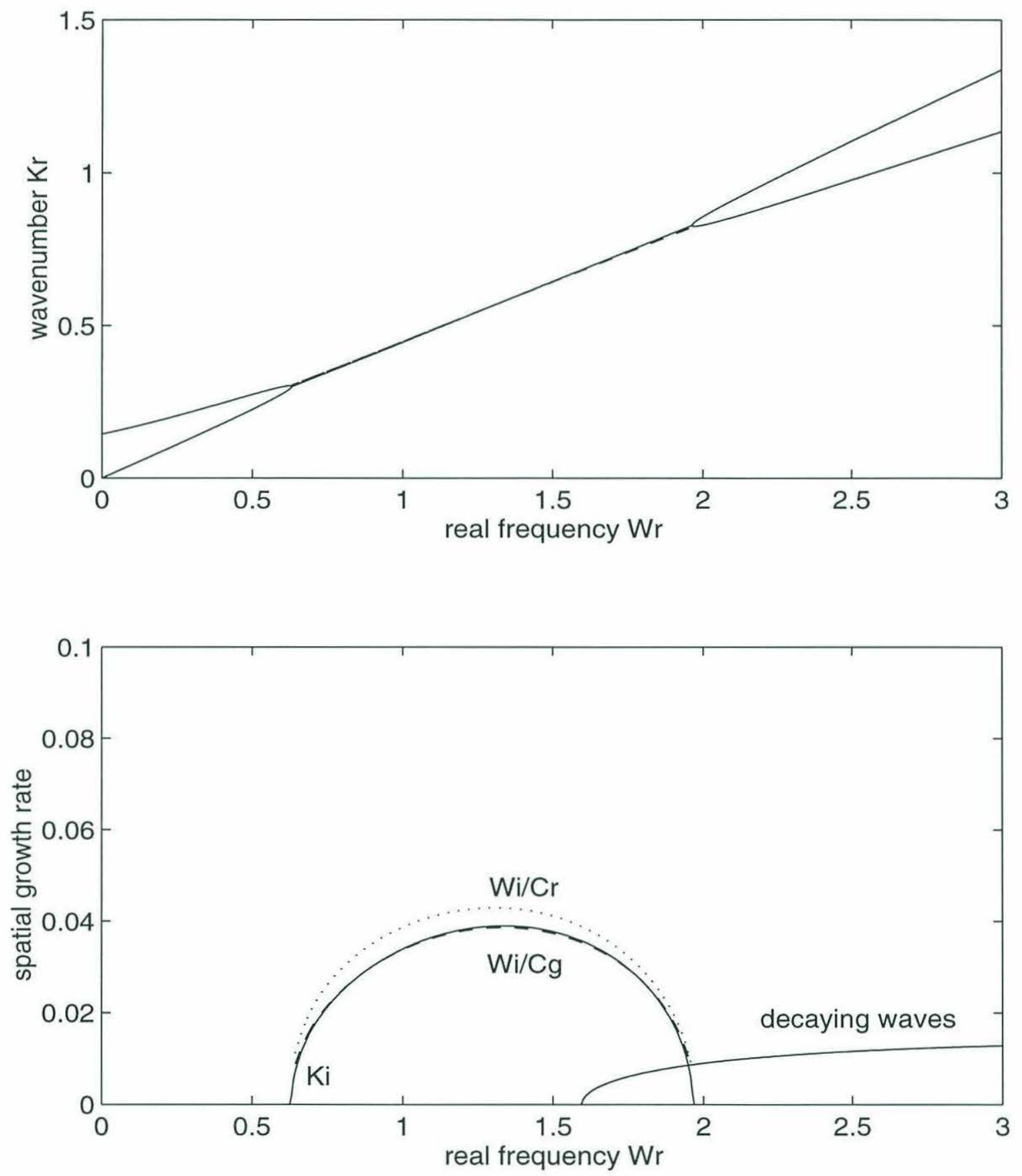

Figure 2.16: Temporal and Spatial, $U_{1}=3, U_{2}=2.2, \beta=0.05$ 

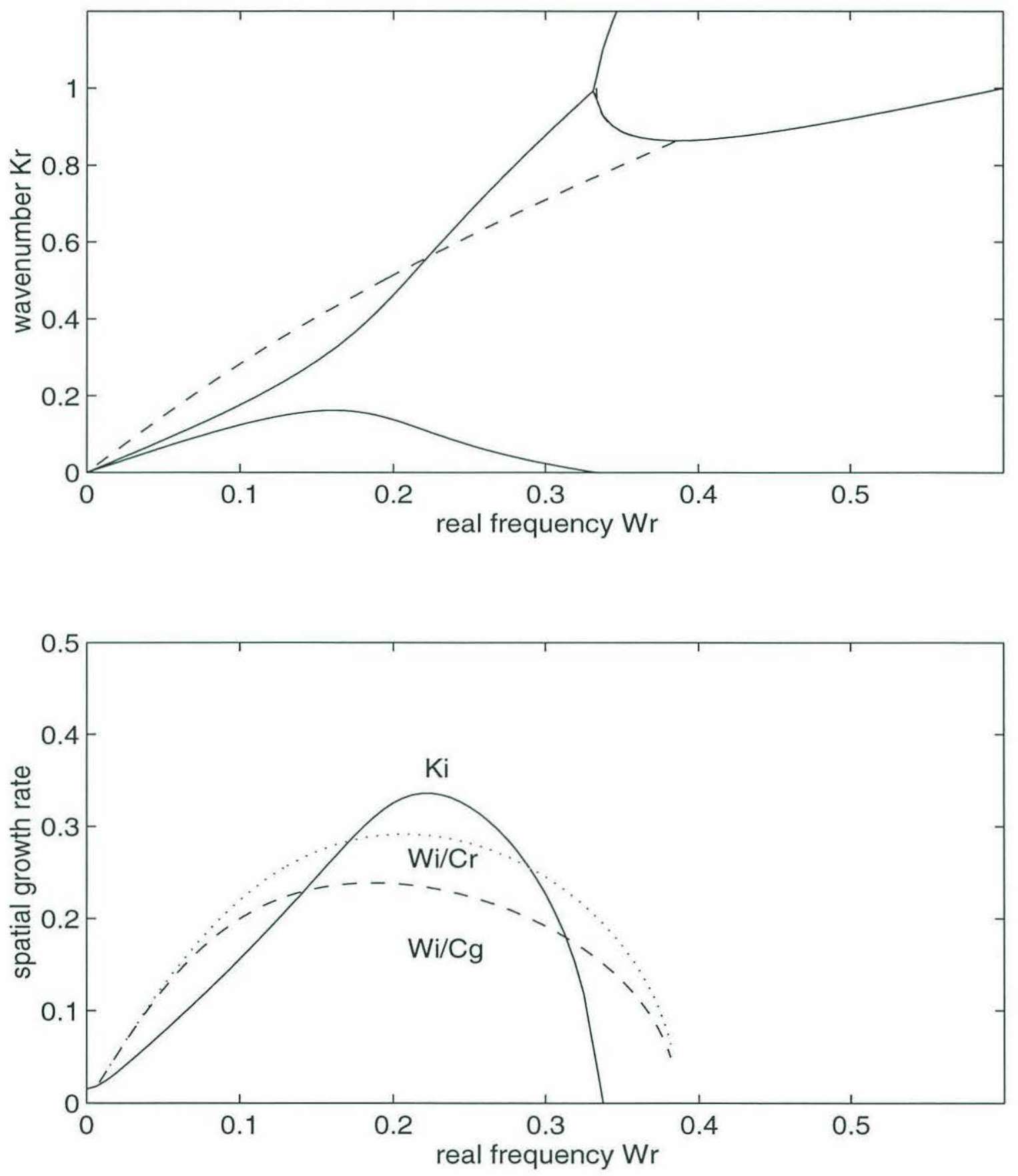

Figure 2.17: Temporal and Spatial, $U_{1}=1, U_{2}=0.2, \beta=0$ 
In Figure 2.18, the growth rates have decreased due to larger $\beta$ (0.13). SGR has been reduced by a factor of 6 in comparison with that in Figure 2.15. However the differences between SGR and ESGR are almost the same in the two figures (ESGR is about $25 \%$ smaller than SGR). To see this more clearly, we keep increasing $\beta$ until it completely stabilizes the flow, and plot the ratio of ESGR to SGR vs beta in the lower panel of Figure 2.19. From the figure, we can see that the ratio of difference (25\%) is almost constant when beta (or maximum growth rate) increases. Also shown in the lower panel is the ratio of PESGR/SGR, which departs widely from 1 when beta is large. In the upper panel of Figure 2.19, we see that the real parts of wavenumbers corresponding to most temporally and spatially unstable waves are very close.

If we increase the mean velocities of basic flow without changing the shear, ESGR will approach SGR (compare Figure 2.15 with Figure 2.16). This can be best seen in the lower panel in Figure 2.20, where ESGR/SGR increases from 0.73 to 0.98 when Umean varies from $1 / 3$ to 2 . Umean is the average of $U_{1}$ and $U_{2}$ weighted by layer depths. This suggests an additional condition on Gaster's transformation formula - the mean flow or the group velocity needs to be fast for it to be valid. Also shown in the lower panel is the ratio of PESGR/SGR, which decreases from 1.35 to 1.12 when Umean varies from $1 / 3$ to 2 , and it does not converge to 1 as fast as the ratio of ESGR/SGR. In the upper panel, again we see that the difference in wavenumbers corresponding to most temporally and spatially unstable waves is very small.

The lower panel of Figure 2.21 shows how ESGR/SGR and PESGR/SGR vary with velocity shear between the top and bottom layers. When the shear increases, ESGR/SGR decreases and departs further away from 1, while PESGR/SGR also decreases but towards 1. From the upper panel, we see that the difference in wavenumbers corresponding to most temporally and spatially unstable waves increases slowly with shear. 

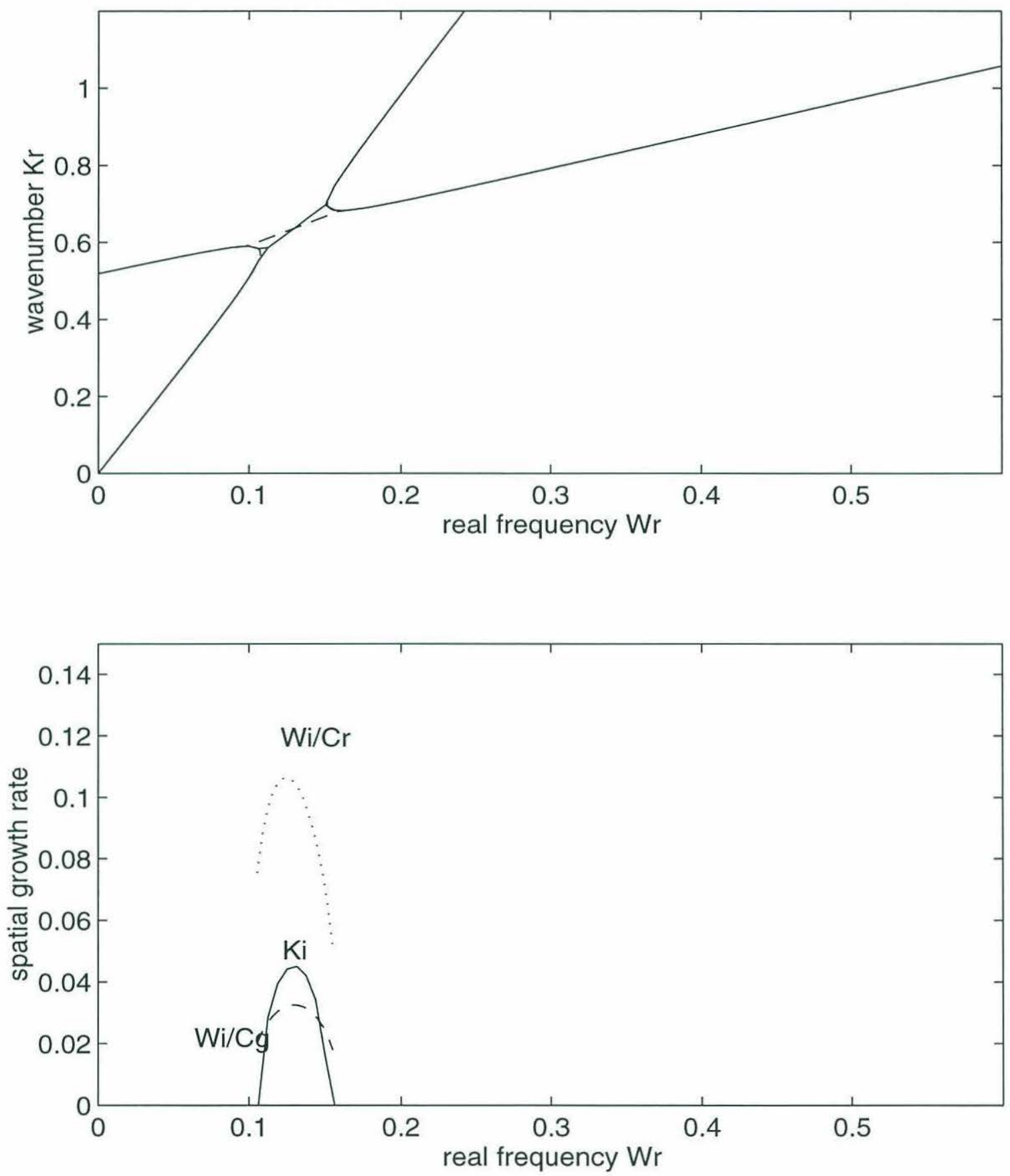

Figure 2.18: Temporal and Spatial, $U_{1}=1, U_{2}=0.2, \beta=0.13$ 

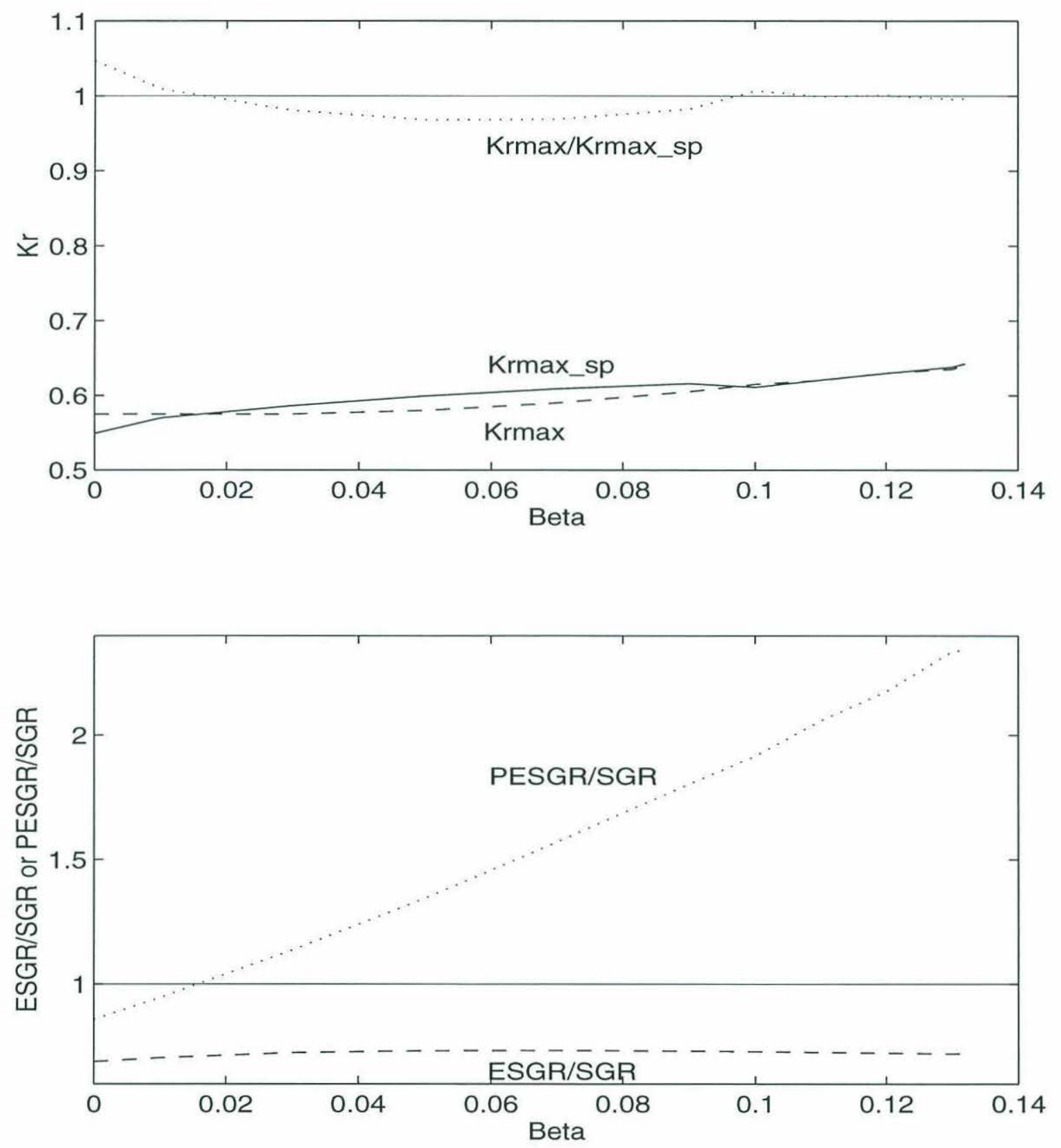

Figure 2.19: Comparison between temporal and spatial instabilities as $\beta$ varies. $U_{1}-U_{2}=0.8$ is fixed. The upper panel shows real parts of wavenumbers corresponding to most temporally and spatially unstable waves and their ratio, and the lower panel ESGR/SGR and PESGR/SGR. 

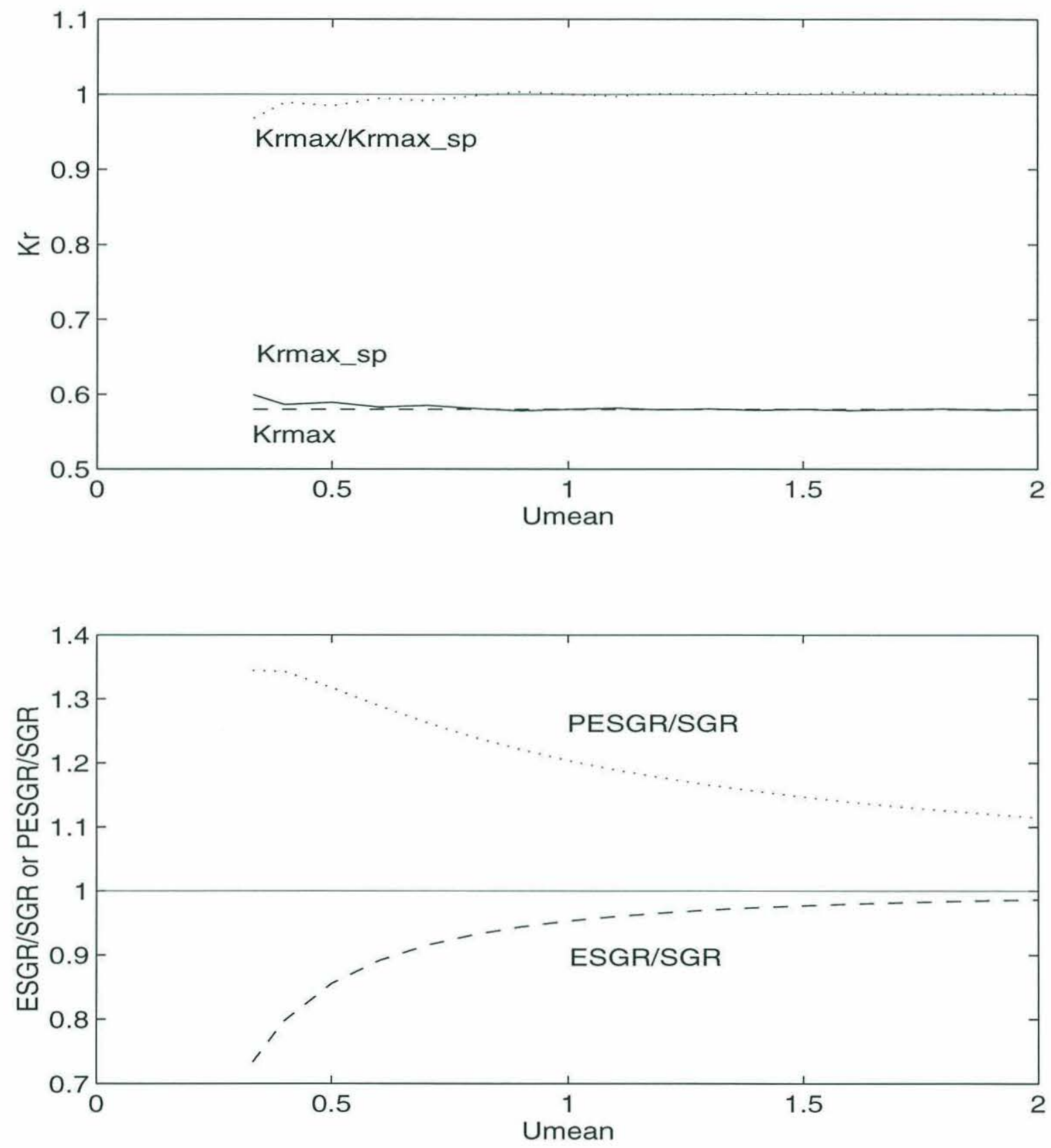

Figure 2.20: Comparison between temporal and spatial instabilities as Umean varies. $U_{1}-U_{2}=0.8$ and $\beta=0.05$ are both fixed. The upper panel shows real parts of wavenumbers corresponding to most temporally and spatially unstable waves and their ratio, and the lower panel ESGR/SGR and PESGR/SGR. 

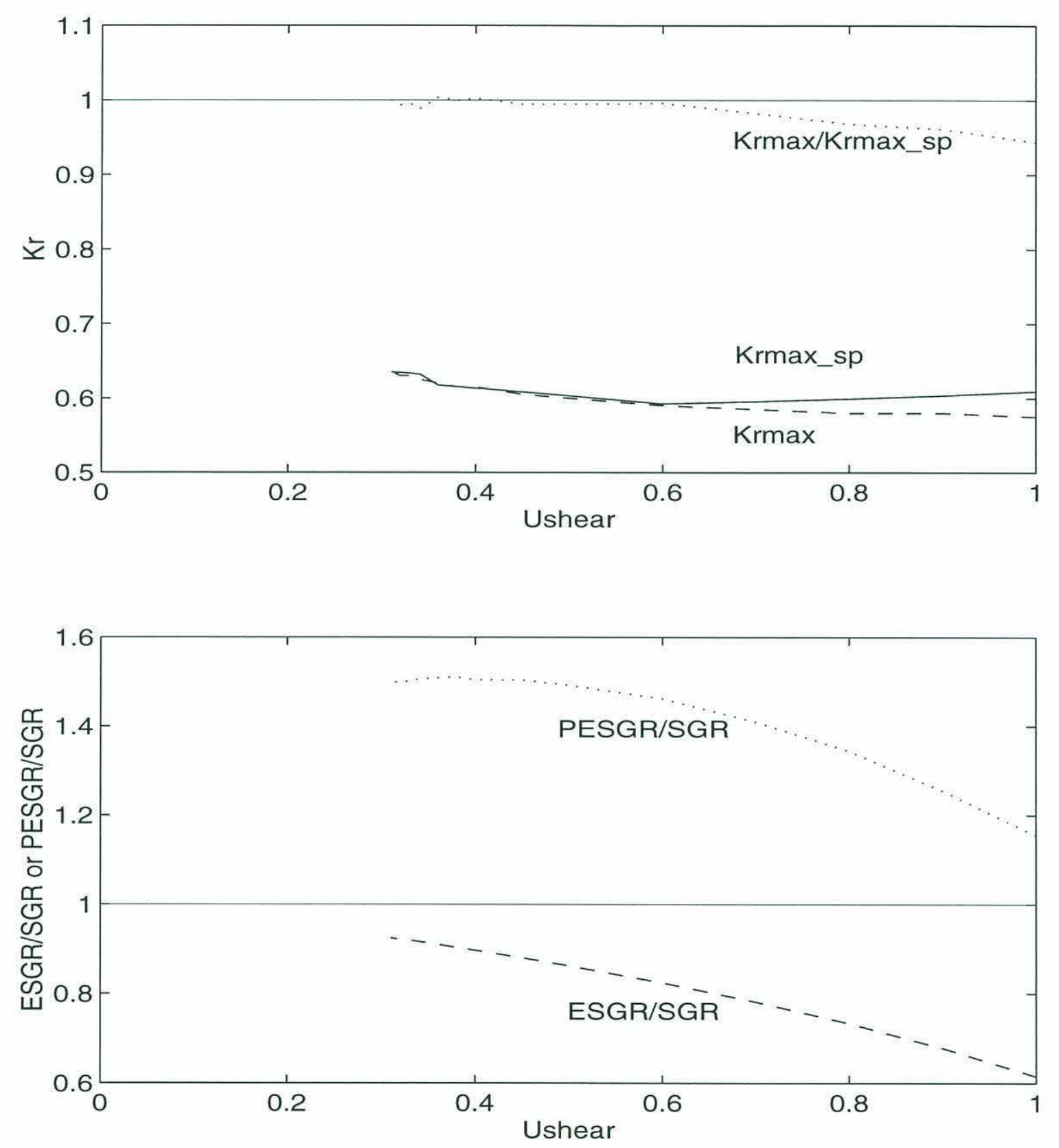

Figure 2.21: Comparison between temporal and spatial instabilities as Ushear varies. $U_{1}-U_{2}=0.8$ and $\beta=0.05$ are both fixed. The upper panel shows real parts of wavenumbers corresponding to most temporally and spatially unstable waves and their ratio, and the lower panel ESGR/SGR and PESGR/SGR. 
In summary, smaller growth rates do not guarantee that ESGR gets closer to SGR. The mean flow or the group velocity needs to be large enough for Gaster's transformation formula to work accurately. When the mean flow is slow, PESGR can depart far away from SGR while ESGR seems to be close to SGR within a bound of 30\%. Small shear helps to keep ESGR close to SGR, while large shear acts to keep PESGR close to SGR. Real parts of wavenumbers (or wavelengths) corresponding to most temporally and spatially unstable waves are comparable and not sensitive to mean velocities, velocity shear and $\beta$.

When the system is continuously forced or only disturbed by a single pulse, which of the three types of instabilities is most relevant needs to be determined. The numerical experiments in the next section address this issue.

\subsection{Numerical Experiments}

In order to test which type of theory is most suitable for a particular situation, a number of experiments have been done in a two-layer spectral numerical model. To derive the governing equation of the model, we separate Equation (2.2.1) into a uniform and purely zonal basic flow $U_{n}$ with stream function $\Psi_{n}(y)$ and a disturbance stream $\psi_{n}$,

$$
\psi_{n}=\Psi_{n}(y)+\phi_{n}(x, y, t)
$$

leading to

$$
\left[\frac{\partial}{\partial t}+U_{n} \frac{\partial}{\partial x}\right] q_{n}+\frac{\partial}{\partial x} \phi_{n} \frac{\partial}{\partial y} \Pi_{n}+\left[\frac{\partial}{\partial x} \phi_{n} \frac{\partial}{\partial y} q_{n}-\frac{\partial}{\partial y} \phi_{n} \frac{\partial}{\partial y} q_{n}\right]=0
$$

where $q_{n}$ is the perturbation potential vorticity,

$$
q_{n}=\nabla^{2} \phi_{n}-F_{n}(-1)^{n}\left(\phi_{2}-\phi_{1}\right)
$$


and $\frac{\partial}{\partial y} \Pi_{n}$ is the potential vorticity gradient of the basic state,

$$
\frac{\partial}{\partial y} \Pi_{n}=\beta-\frac{\partial^{2}}{\partial y^{2}} U_{n}-F_{n}(-1)^{n}\left(U_{1}-U_{2}\right)
$$

Linearizing and adding forcing gives

$$
\left[\frac{\partial}{\partial t}+U_{n} \frac{\partial}{\partial x}\right] q_{n}+\frac{\partial}{\partial x} \phi_{n} \frac{\partial}{\partial y} \Pi_{n}=F(x, t)=G_{n}(x) f(t)
$$

with lateral boundary conditions,

$$
\frac{\partial}{\partial x} \phi_{n}=0 \quad y=0, L_{y}
$$

The forcing is introduced at a particular location $x_{0}$ with Gaussian distribution $G_{n}(x)=A_{n} e^{-\left(x-x_{0}\right)^{2} / L_{f}^{2}}$ in a small region and time dependence $f(t)=\sin \left(\omega_{f} t\right)$. The forcing scale $L_{f}$ and frequency $\omega_{f}$ can be varied. Only the gravest mode in $y$ direction was retained in the experiments.

\section{Pulse Disturbance}

In the experiments concerning evolution of a pulse disturbance, I set $F(x, t)$ in Equation (2.5.19) to be 0 and $\phi_{n}=G_{n}(x)=A_{n} e^{-\left(x-x_{n}\right)^{2} / L_{f}^{2}}$ at $t=0 . A_{1}$ is chosen to be $2 A_{2}$ and is offset by 1 Rossby deformation radius to the west arbitrarily, i.e., $x_{1}=x_{2}-1 . L_{f}$ is 0.6 . The upper panel in Figure 2.22 shows the initial condition. The $x$ axis was labeled with positions of grid points. The lower panel shows a general picture of the developed disturbance after some time.

Figure 2.23 shows the amplitudes of disturbances at a particular point. Solid and dashed curve are values of the stream functions at the specified point in the upper and lower layers, respectively. Whether the system is absolutely or convectively unstable becomes clear after the peak of envelope pass that point. The upper and lower panels are from two separate numerical runs to show the difference between absolute and convective instabilities. The upper panel corresponds to absolute instability, while the lower one displays convective instability. 

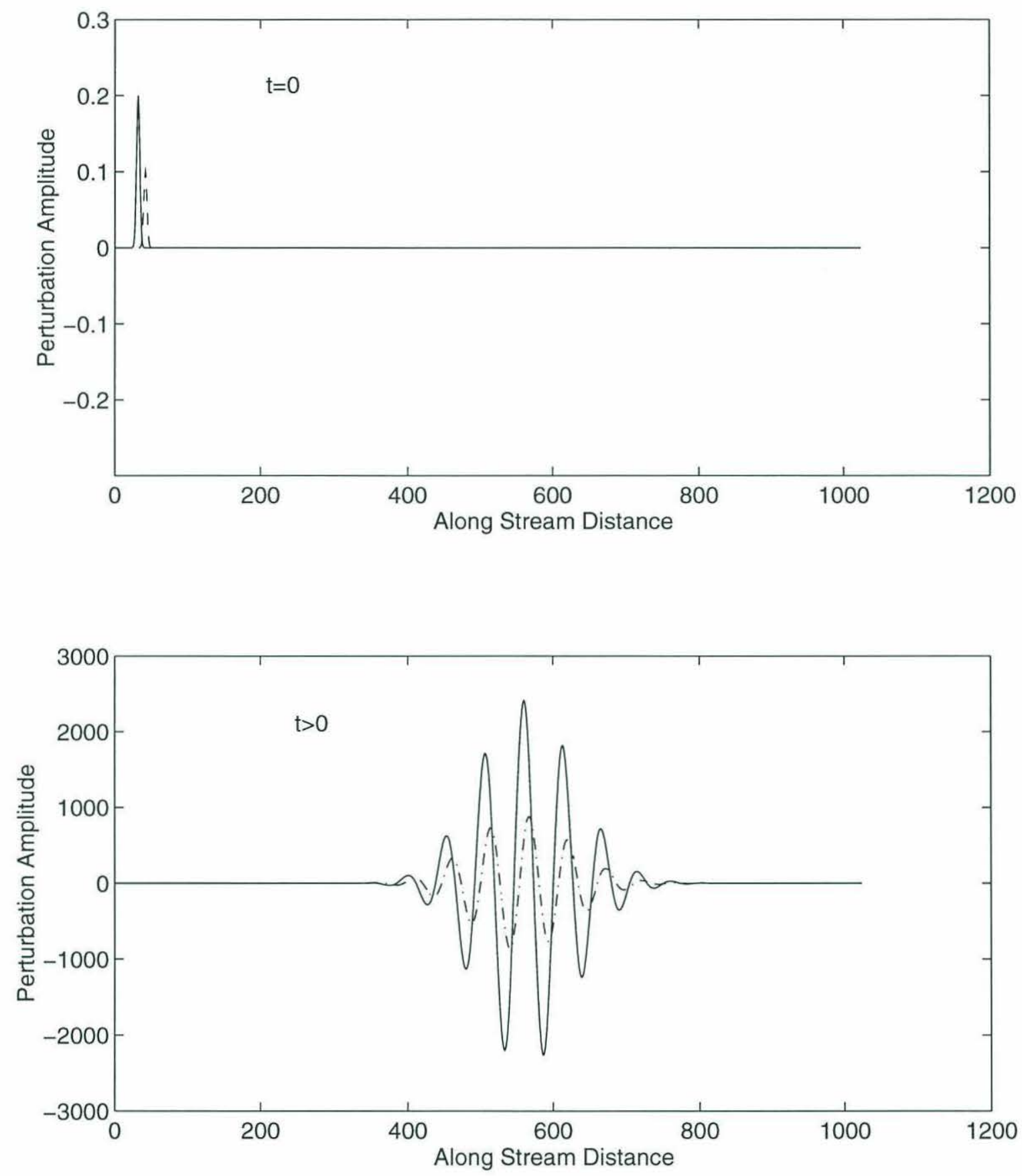

Figure 2.22: Pulse instability. Upper panel: the initial condition used. Lower panel: a typical well developed wave packet. Solid and dashed curves represent the stream functions in the upper and lower layers respectively. 

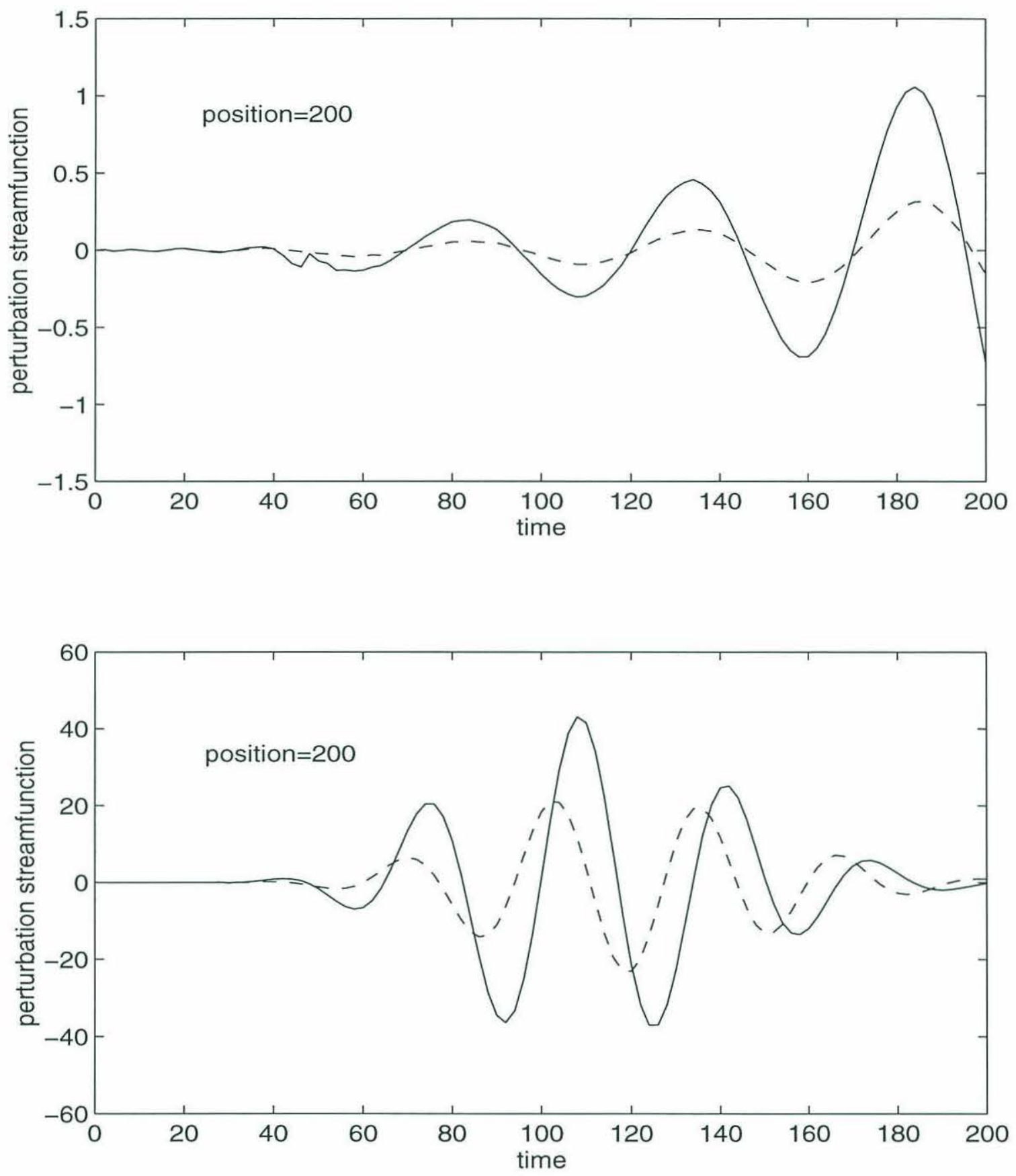

Figure 2.23: Value of $\psi$ vs. time at a specified point. The upper and lower panels are from two separate numerical runs to show the difference between absolute and convective instabilities. Upper panel: absolute instability; Lower panel: convective instability. 
Figure 2.24 demonstrates how to calculate the growth rate of a wave envelope. Panel a shows a well developed wave packet at time t. The envelope is determined by picking out the crests and troughs and using spline method to interpolate the amplitudes of the disturbance between crests and between troughs. These amplitudes form the envelope of a wave packet. We then rescale the horizontal coordinate by $\mathrm{t}$ and transform panel a to panel b. The coordinate in panel b is $C_{g}=\frac{x}{t}$. Panel $\mathrm{c}$ shows the wave packet at $t+1$. Again, we transform the envelope in panel $c$ to panel $\mathrm{d}$ (outer envelope) by rescale the $\mathrm{x}$ axis. The coordinate in panel $\mathrm{d}$ is $C_{g}=\frac{x}{t+1}$. Panel $\mathrm{d}$ also draws the envelope from panel b (inner envelope) for direct comparison. From panel d, we calculate the growth rate of the envelope for each $C_{g}$ from the amplitudes of the two envelopes. The growth rate is then plotted in panel e.

We can follow the similar procedure to calculate growth rates of individual peaks, as shown in Figure 2.25. In panel a-d, the dots mark individual crests and troughs in the wave packet. The rescaling procedure is the same as described above for the calculation of the envelope growth. First convert wave packets at time $t$ and $t+1$ (panel a and c) from $\mathrm{x}$ coordinates into group velocity coordinates (panel $\mathrm{b}$ and d). In panel $\mathrm{d}$, the corresponding peaks at $t$ and $t+1$ are connected by solid lines. We then measure the amplitude difference of each pair and divide it by the time step to get the growth rate of each peak. The growth rates of individual peaks are then plot in panel e with (*)'s. Note that near the both edges of the wave packet, the amplitudes of waves are small compared to the maximum of the envelope (panel d), so that the relative error in the measured amplitudes near both edges may be larger than that near the center of the packet due to numerical noises. Therefore the resulting error in the growth rates of peaks near the edges may be larger than that near the center.

(*) $\mathrm{s}$ in the upper panel of Figure 2.26 show such determined growth rates of individual peaks from a numerical run. Also drawn in the panel is the growth rate 

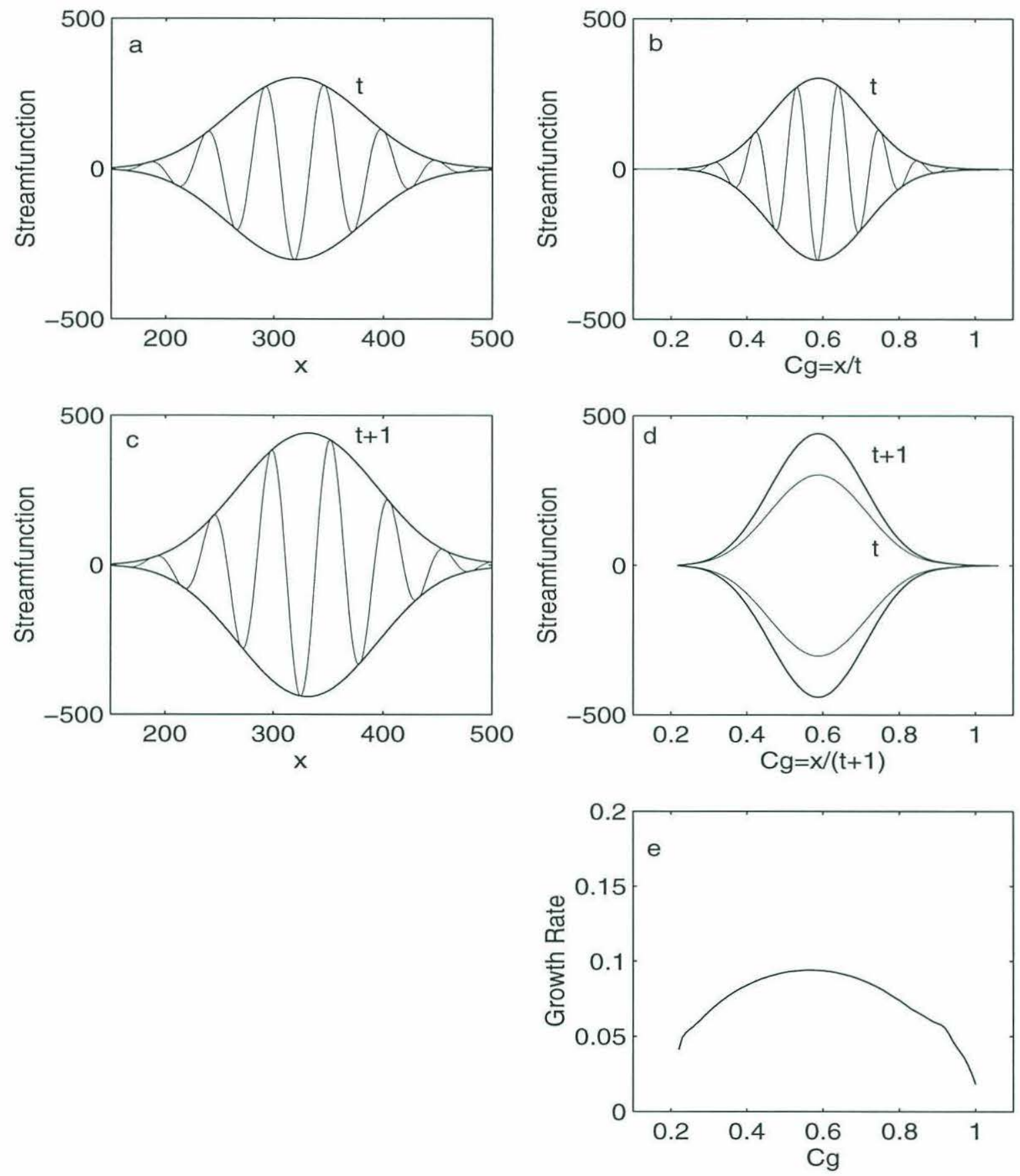

Figure 2.24: Demonstration: how the growth rate of the envelope is calculated in a numerical run. 

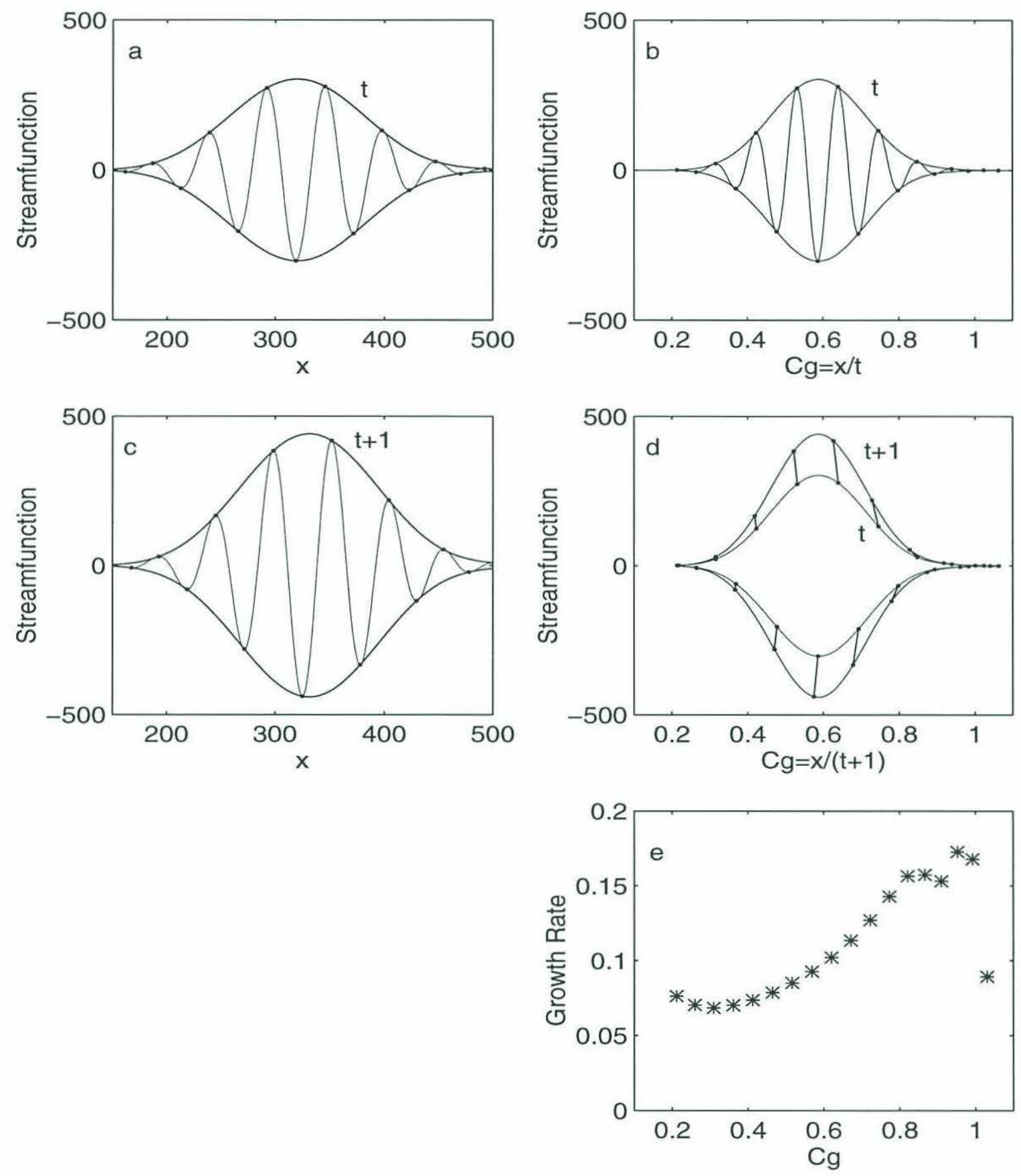

Figure 2.25: Demonstration: how the growth rate of an ind. peak is calculated in a numerical run. 
of an individual peak (dark dashed curve) from an analytical model with the same parameters.

In the lower panel, solid curve indicates the growth rate of the envelope by the method demonstrated in Figure 2.24. Dashed curve represents the growth rate of the envelope from analytical model with the same parameters.

The growth rates from numerical models are slightly lower than their analytical counterparts; for example, the maximum growth rate of the envelope in the numerical run is $4.5 \%$ lower than that in analytical model. However, if we subtract $\frac{1}{2 t}$ from the latter, the correction term in Equation (2.3.14), the difference is then only $0.17 \%$. Hence the correction term is responsible for $96 \%$ of the discrepancy in growth rates of the most unstable waves between the numerical and analytical models. Moreover, the discrepancy seems fairly uniform over a large range of $C_{g}$ in the middle and is somewhat irregular near both ends of the group velocity range. There are two plausible reasons; the first is that in the leading and trailing edges, the numerical noise may be more significant because of small wave amplitudes there. The second is that the asymptotic solution may break down when group velocity is near its maximum or minimum point, as discussed in Section 2.3.3. This is less likely than the first reason because the irregularity in the discrepancy is mainly due to the irregularity in the numerical curve.

In the derivation of the growth rate of the envelope in Section 2.3.3, I have assumed that when $\omega(k)$ have branch points, we can avoid going around these points in some way when we integrate to get asymptotic solution. It is difficult to prove this assumption mathematically. However, the reasonable agreement with numerical results demonstrates that this is a sensible assumption.

Comparing the upper and lower panels in Figure 2.26, we again see that wave in the leading edge can grow much faster than the maximum of the envelope 
and waves behind the maximum of the envelope grow slower than the corresponding envelope. The relative position of an individual peak to the maximum of the envelope determines its growth rate and other properties such as wavenumber and frequency.

The upper and lower panels of Figure 2.27 show the corresponding wavenumber and frequency from both numerical and analytical models, and they also seem to agree very well except slight discrepancies at the low group velocity end, probably for the same reasons mentioned above. The wavenumber is estimated via the half wavelength, which is calculated by measuring the positions of an adjacent crest and trough and the value is assigned to the average position of the two points.

When a single pulse evolves as pulse instability predicts, each component of a specific wavenumber in energy spectrum still grows according to the growth rate of temporal instability. This is naturally true mathematically. However I emphasize it here for future reference by a demonstration in Figure 2.28, which compares the growth rate of each component measured in a numerical run (marked with ' $x$ ') and the growth rate calculated from temporal instability theory (solid curve). Therefore when pulse instability occurs, the most energetic wave is the most temporally unstable wave.

In preparation of comparison with observations in Chapter 4, I have also performed 2D spectral analysis on the above numerical run. The purpose is to demonstrate that the most energetic wave picked by $2 \mathrm{D}$ spectral analysis is the temporally most unstable wave. I took the stream function in the upper layer, which is a function of both position $x$ and time $t$ and transformed it into wavenumber and frequency domain by 2D FFT. Figure 2.29 shows the energy spectrum of such an analysis. The most energetic wave from the spectrum has a wavenumber of 0.583 and a frequency of 0.181 , while the most unstable wave from analytical model has $\omega=0.580$ and $k=0.179$. The discrepancies in the wavenumber and frequency are already smaller than the minimum wavenumber and frequency the 2D spectral analysis can resolve, 

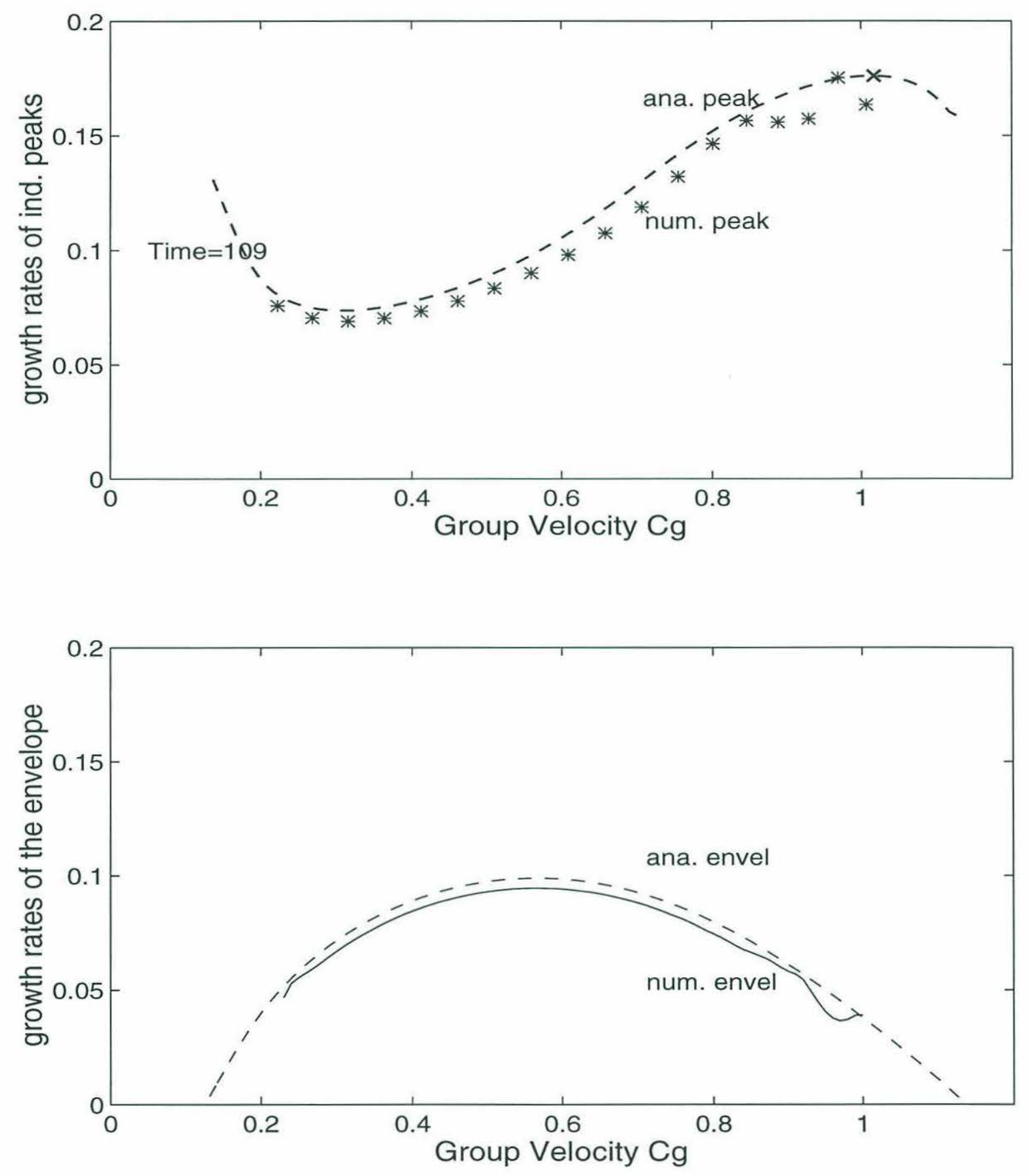

Figure 2.26: Comparison of numerical results with analytical results. Time $=109$. 

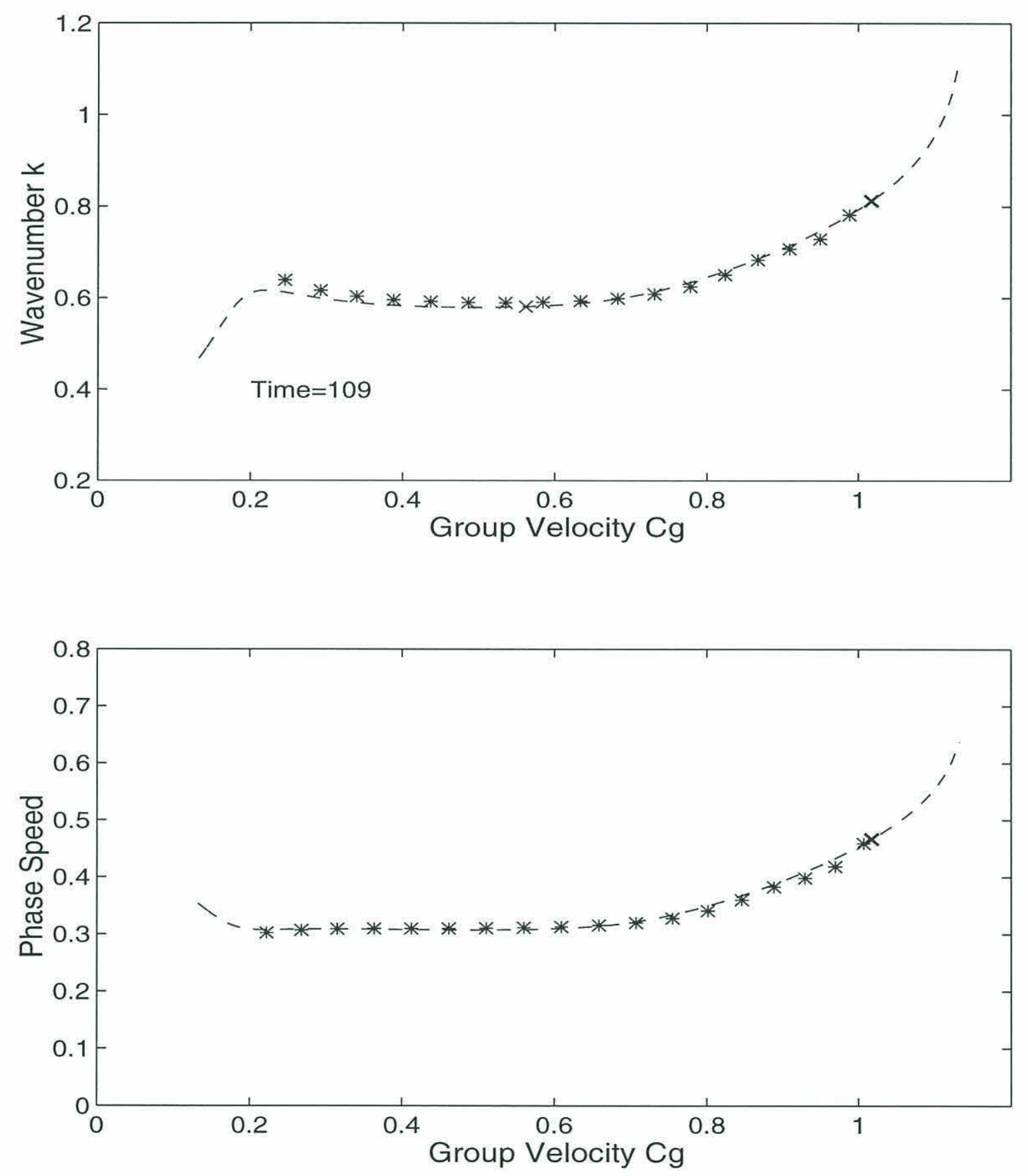

Figure 2.27: Comparison of numerical results with analytical results. 


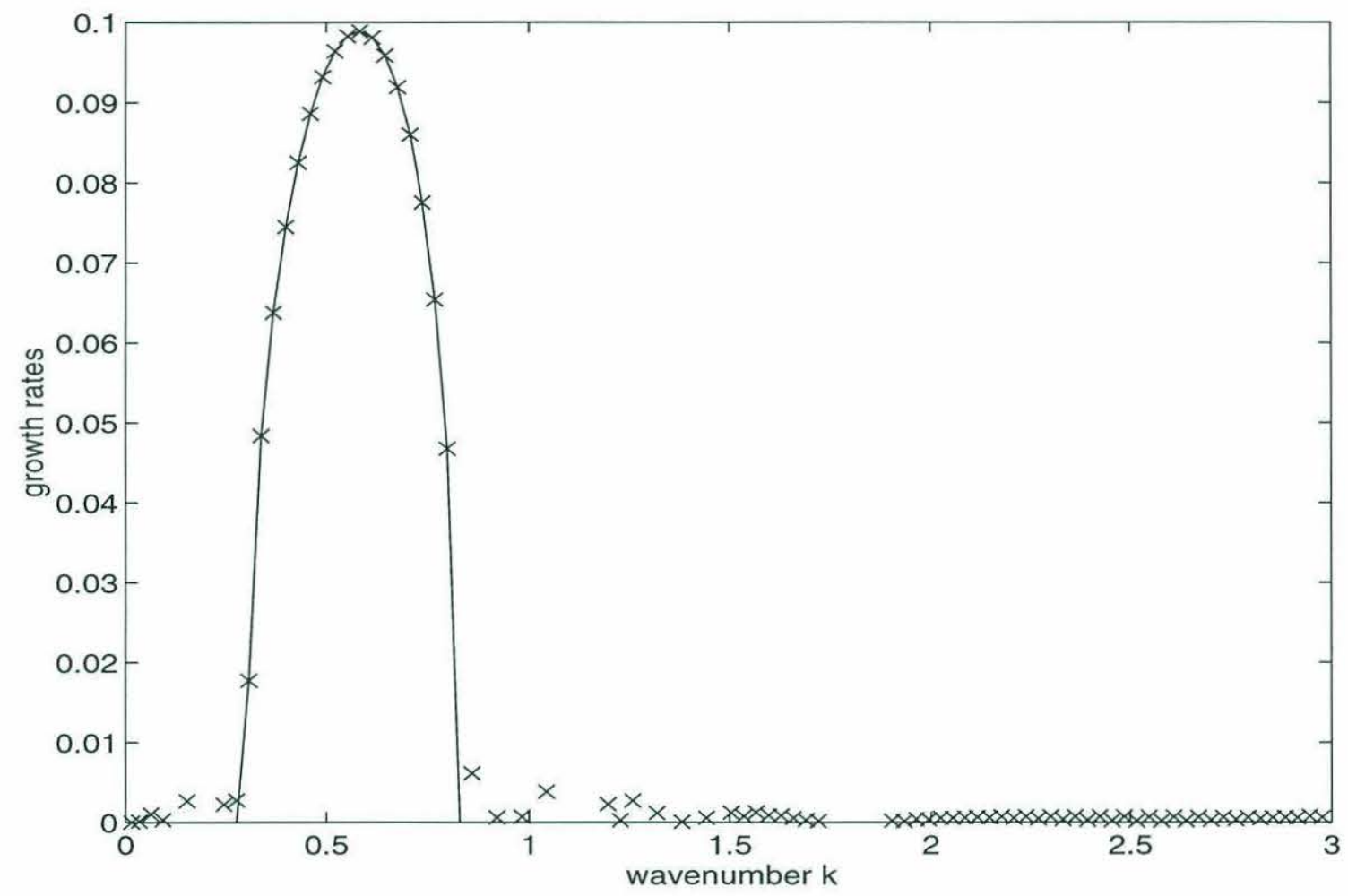

Figure 2.28: Solid curve: the growth rate from temporal instability. ' $x$ ': the growth rate of a component at a specific wavenumber measured from a pulse instability numerical run. Model parameters: $U_{1}=1.0, U_{2}=0.2$ and $\beta=0.05$. 


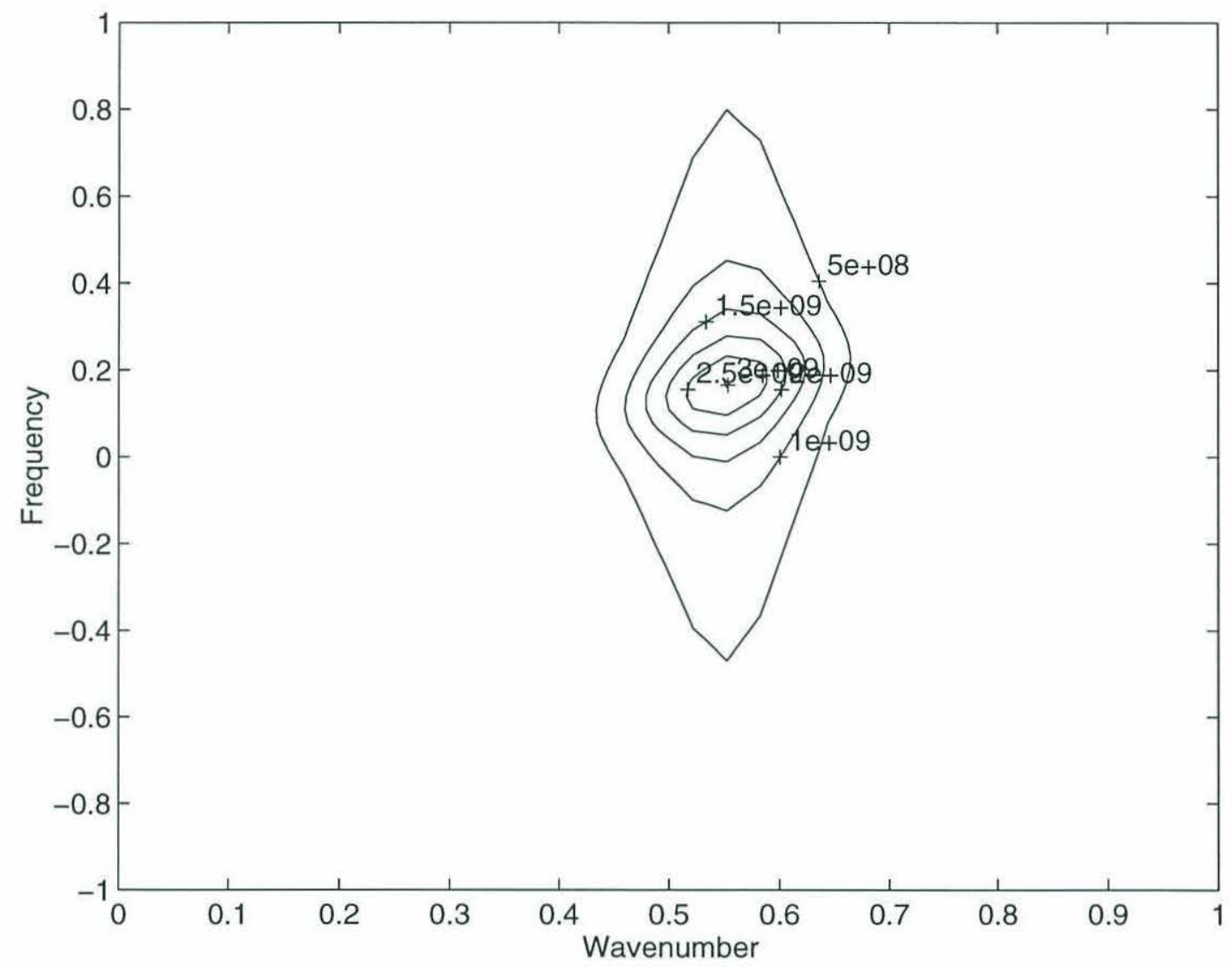

Figure 2.29: Energy spectrum from the above numerical run (pulse instability). Model parameters: $U_{1}=1.0, U_{2}=0.2$ and $\beta=0.05$.

because of the finite length of time series and the finite model domain. Hence we conclude that in the case of the pulse instability, the most energetic wave, picked from $2 \mathrm{D}$ spectral analysis, is the temporally most unstable wave within the analysis error.

\subsubsection{Single frequency forcing}

When a system is continuously forced at constant frequency, a spatial instability is expected to happen. Only when the forcing frequency is within the unstable range predicted by spatial instability theory, can the system be spatially unstable. When the forcing is out of the spatially unstable range, only the initial peak of the 


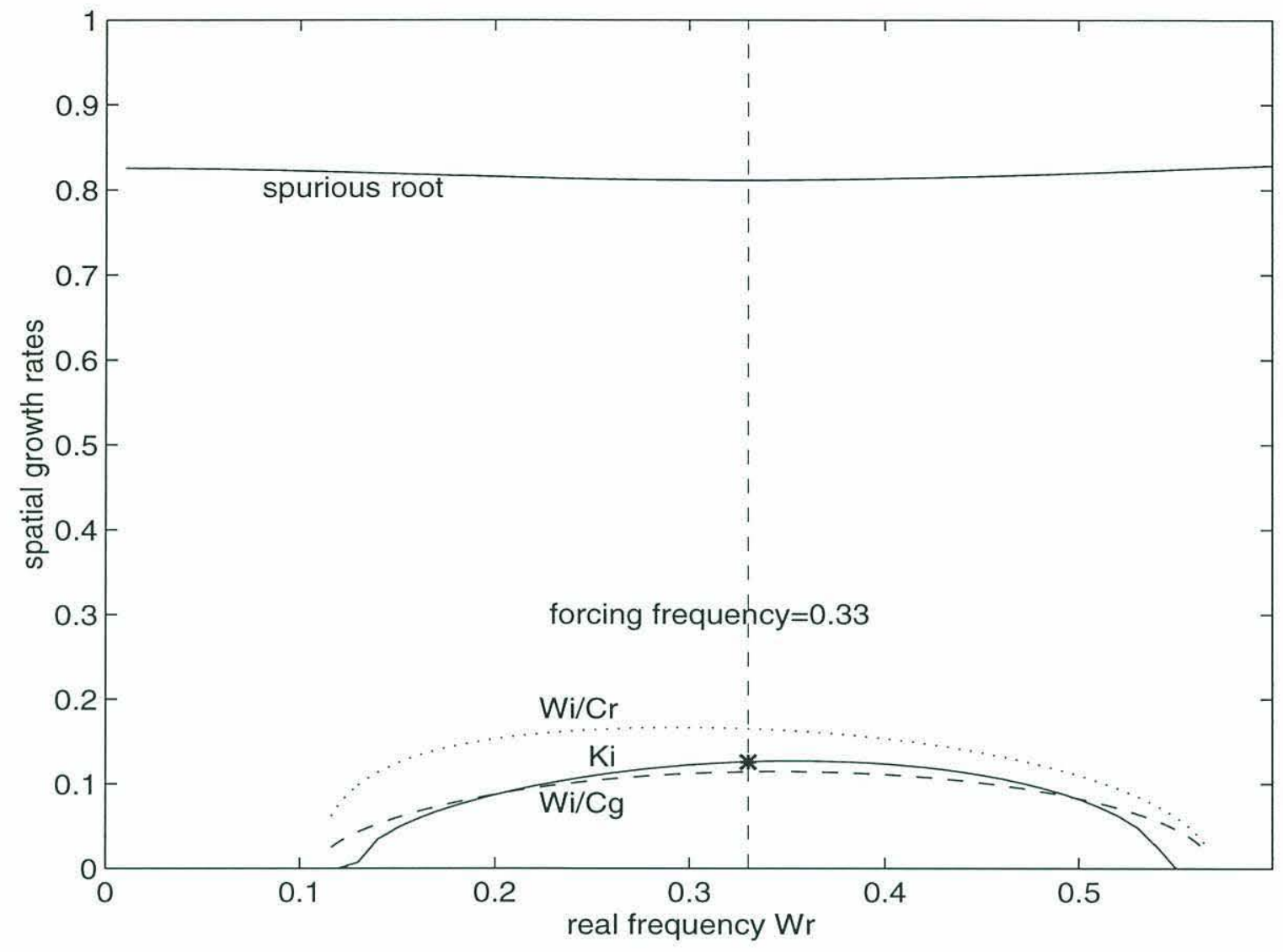

Figure 2.30: Spatial growth rate and equivalent spatial growth rate, $U_{1}=1.3, U_{2}=0.5, \beta=0.05$. '*' indicates the numerical result. vertical dashed line shows the forcing frequency.

disturbance can grow convectively as pulse instability theory predicts. Behind the initial peak, destructive interaction between pulses prevents growth of perturbation.

As we can see from Figure 2.30 or Figure 2.15, the spatially unstable range is narrower than temporally unstable range. This means that when the system is forced continuously at a frequency within the temporal instability range but out of spatial instability range, other types of instability such as convective instability will occur instead of spatial instability.

When spatial instability happens, the amplitude at a particular point becomes constant. The spatial growth rate can be determined by measuring amplitudes at two points. As an example, for the parameters shown in Figure $2.30\left(U_{1}=1.3\right.$, 
$U_{2}=0.5, \beta=0.05$ ), a continuous forcing was introduced with a constant frequency 0.33 , the spatial growth rate measured from numerical run is 0.1257 , which is marked with ' $*$ ' in Figure 2.30, while spatial instability theory predicted a growth rate of $0.1262(0.4 \%$ higher $)$ and the temporal instability theory yielded an equivalent spatial growth rate of 0.1154 ( $8.2 \%$ lower). So the numerical spatial growth rate agrees well with spatial instability theory as expected when the system is continuously forced at constant frequency. Note that the other analytical root with large spatial growth rate identified as spurious solution by Briggs' rule (see Section 2.3.2) indeed does not show up in the numerical experiment.

\subsection{Summary}

The first part of this chapter has introduced basic concepts of temporal, spatial, pulse, absolute and convective instabilities which have already appeared in the literature. The calculations herein are similar to others but show all three kinds of instabilities using the same model and parameter values. I will summarize in the following subsections the results from the literature and from this work which are most relevant to the further work in the later chapters.

\subsubsection{Temporal Instability}

Briggs (1964) proved that for any system to support unstable waves, its dispersion equation must yield complex solutions with positive imaginary parts for some real $k$, i.e. it is a necessary condition for any unstable waves. This implies that spatial and pulse instabilities only occur when temporal instability also occurs. 


\subsubsection{Spatial Instability}

Briggs also shows that not all solutions with the right sign for spatial instability are growing waves. We need to apply Briggs criterion (described in Section 2.3) to distinguish real growing waves from other solutions with the same sign. I have used the criterion to say that implication in Hogg's (1976) work that spatial instability does not require potential vorticity gradients to change sign is not legitimate. In addition, I have shown that the purely spatially unstable wave Hogg considered is really better thought of as neutral because its phase speed is real. Hence one of his conclusions, that spatially growing waves are bounded by a low wavenumber cut-off which separates them from the temporally growing waves, is not valid. That conclusion has been drawn based on nonzero $c$ although he focuses on the limit of zero $c$ in his study and it is not clear whether or not $c=0$ also corresponds to a neutral wave.

Michalke (1965) first studied the difference between spatial growth rate (SGR) and pseudo-equivalent spatial growth rate (PESGR). He found that PESGR was $17 \%$ smaller than SGR in a barotropic flow with a hyperbolic tangent velocity profile. In this chapter, I have made comparisons among SGR, PESGR and ESGR (the equivalent spatial growth rate) and examined the validity of Gaster's formula in the Phillips model.

Spatial instability theory gives different and generally larger spatial growth rates than the equivalent spatial growth rate (ESGR) transformed from temporal instability with Gaster's formula, especially when growth rate is large and group velocity is small. Smaller growth rates do not guarantee that ESGR gets closer to SGR.

The mean flow or the group velocity needs to be large enough for Gaster's transformation formula to work accurately. When the mean flow is slow, PESGR can 
depart far away from SGR while ESGR seems to be close to SGR within a bound of 30\%. Small shear helps to keep ESGR close to SGR.

Real parts of wavenumbers (or wavelengths) corresponding to most tempo-

rally and spatially unstable waves are comparable and not sensitive to mean velocities, velocity shear and $\beta$.

\subsubsection{Pulse Instability}

The maximum of the envelope of the growing disturbance moves at group velocity of the most temporally unstable wave, and grows at the rate of the same wave. The growth rate of an individual peak depends on the location of the peak relative to the maximum of the envelope. Given $C_{g}>C_{p h}$, individual peaks behind the maximum of the envelope grow at a rate smaller than $\omega_{\text {imax }}$. At the leading edge, individual peaks can grow much faster than the peak of the envelope, which makes the envelope move faster than the individual peaks. These general results were first found by Simmons and Hoskins (1979) in atmospheric observation. The formula of growth rate of an individual peak was also given in the paper without a mathematical derivation. In this chapter, I have provided a detailed theoretical derivation of the formula and focused on the behaviors of individual peaks. In particular, when the growth rate of an individual peak is plotted as a function of its amplitude, it decreases as the amplitude increases. It has been demonstrated in this work that the relation between the growth rate of an individual peak and its amplitude is an intrinsic property and an important feature of pulse instability. Thus if one follows a single peak and notices a decrease in the growth rate, one cannot infer that increasing amplitude (or nonlinearity) was responsible for reducing the growth rate.

Direct comparison between temporal and pulse theory in this chapter shows that pulse instability allows unstable waves to have wavenumbers beyond the short 
wave cut-off of the temporal theory. This is reasonable because we are comparing the real part of the local wavenumber in pulse theory with global wavenumber in temporal theory. The local wavenumber in pulse theory contains a nonzero imaginary part, so that even if its real part is the same as the wavenumber in temporal theory, its frequency $\omega\left(k_{r}+i k_{i}\right)$ has been extended to complex $k$ plane and is naturally different from that in temporal theory $\left(\omega\left(k_{r}\right)\right)$.

When a single pulse evolves as pulse instability predicts, each component of a specific wavenumber in energy spectrum still grows according to the growth rate of temporal instability. Therefore when pulse instability occurs, the most energetic wave is the most temporally unstable wave. 


\section{Chapter 3 \\ The Instabilities of the Gulf Stream-QG Models}

\subsection{Motivation}

From the two-layer model discussed in the last chapter, we have already seen that there can be significant differences between temporal, pulse and spatial instabilities, and Gaster's transformation formula is valid only when a spatial growth rate is small and the group velocity is large. In order to answer the question whether Gaster's formula and the other transformation formulae commonly used hold well in the context of the Gulf Stream, we have to consider models more realistic than the two-layer uniform velocity model we have studied.

There are several ways we can improve the model used in the last chapter. First, a jet structure in the basic state may have significant effect on the instabilities. Second, topography is expected to modify instabilities in some ways (Orlanski, 1969). Third, we want to know the effect of additional layers or more detailed stratification. Fourth, it is more reasonable to use a shallow water model than a QG model because the slopes of isopycnals and topography and the Rossby number are large in the context of the Gulf Stream.

In this chapter, we first consider a six-layer QG model with a jet structure basic flow and with relatively realistic topography. We then proceed to compare the six-layer QG model with two to five-layer models to examine the effect of vertical resolution. Our study shows that a minimum of three layers is necessary to properly address the effect of topography and at least five layers are required to capture the 
rapid change in velocity profile (see Section 3.3). Hence a six-layer model is desirable in this regard.

The variations in topography and thicknesses of layers between isopycnals in the Gulf Stream are large enough that the conditions for QG theory to be formally valid are violated. In the next chapter, I will examine a shallow water model with the same topography and jet structure to determine how the predictions of the QG model are changed. 


\subsection{Model Description}

We will use two to six layer quasi-geostrophic inviscid channel models in this chapter. These models are identical in dynamics except for the different number of layers and can be classified as a multiple layer model. The stream function in each layer is independent of depth. Let $n=1$ indicate the top layer, and $n=N$ the bottom one. Based on layer models described in Pedlosky (1986, Sec. 6.16), the equations of motion for a $\mathrm{N}$ layer model can be written

$$
\begin{aligned}
& \left\{\frac{\partial}{\partial t}+\frac{\partial}{\partial x} \psi_{1} \frac{\partial}{\partial y}-\frac{\partial}{\partial y} \psi_{1} \frac{\partial}{\partial x}\right\}\left\{\nabla^{2} \psi_{1}-F_{1,0}\left(\psi_{1}-\psi_{2}\right)+\beta y\right\}=0 \\
& \left\{\frac{\partial}{\partial t}+\frac{\partial}{\partial x} \psi_{n} \frac{\partial}{\partial y}-\frac{\partial}{\partial y} \psi_{n} \frac{\partial}{\partial x}\right\}\left\{\nabla^{2} \psi_{n}+F_{n-1, n}\left(\psi_{n-1}-\psi_{n}\right)+\right. \\
& \left.+F_{n, n}\left(\psi_{n+1}-\psi_{n}\right)+\beta y\right\}=0, \quad 1<n<N \\
& \left\{\frac{\partial}{\partial t}+\frac{\partial}{\partial x} \psi_{N} \frac{\partial}{\partial y}-\frac{\partial}{\partial y} \psi_{N} \frac{\partial}{\partial x}\right\}\left\{\nabla^{2} \psi_{N}-F_{N-1, N}\left(\psi_{N}-\psi_{N-1}\right)+\right. \\
& \left.+\beta y+f_{0} \eta_{B} / H_{N}\right\}=0
\end{aligned}
$$

where $\beta$ is the gradient of planetary vorticity and $\eta_{B}$ the topography. Fs are Froude numbers,

$$
F_{m, n}=\frac{f_{0}^{2} L^{2}}{\epsilon_{m} g H_{n}}
$$

where $f_{0}$ is Coriolis force at $38^{\circ} \mathrm{N}, \epsilon_{m}$ the nondimensional density jump between m-th and $(\mathrm{m}+1)$-th layers and $H_{n}$ the depth of $\mathrm{n}$-th layer. $L$ is the length scale. $F_{0,1}$ and $F_{N, N}$ are defined to be 0 .

The standard linear instability theory for a basic state with zonal flows $U_{n}(y)$ and perturbations $\phi_{n}=\operatorname{Re}\left(\Phi_{n}(y) e^{i k(x-c t)}\right)$ gives the eigenvalue problem

$$
\begin{aligned}
& \left(U_{1}-c\right) \quad\left[\frac{\partial^{2}}{\partial y^{2}} \Phi_{1}-k^{2} \Phi_{1}-F_{1,0}\left(\Phi_{1}-\Phi_{2}\right)\right]+\Phi_{1} \frac{\partial}{\partial y} \Pi_{1}=0 \\
& \left(U_{n}-c\right)\left[\frac{\partial^{2}}{\partial y^{2}} \Phi_{n}-k^{2} \Phi_{n}+F_{n-1, n}\left(\Phi_{n-1}-\Phi_{n}\right)+F_{n, n}\left(\Phi_{n+1}-\Phi_{n}\right)\right]+
\end{aligned}
$$




$$
\begin{gathered}
+\Phi_{n} \frac{\partial}{\partial y} \Pi_{n}=0, \quad 1<n<N \\
\left(U_{N}-c\right)\left[\frac{\partial^{2}}{\partial y^{2}} \Phi_{N} \quad-k^{2} \Phi_{N}-F_{N-1, N}\left(\Phi_{N}-\Phi_{N-1}\right)\right]+\Phi_{N} \frac{\partial}{\partial y} \Pi_{N}=0
\end{gathered}
$$

with the boundary condition

$$
\Phi_{n}=0, \quad y=0, L_{y}, \quad n=1, \ldots, N
$$

where $\frac{\partial}{\partial y} \Pi_{n}$ is the potential vorticity gradient of the basic state,

$$
\begin{aligned}
\frac{\partial}{\partial y} \Pi_{1} & =\beta-\frac{\partial^{2}}{\partial y^{2}} U_{1}-F_{1,0}\left(U_{2}-U_{1}\right) \\
\frac{\partial}{\partial y} \Pi_{n} & =\beta-\frac{\partial^{2}}{\partial y^{2}} U_{n}-F_{n-1, n}\left(U_{n-1}-U_{n}\right)-F_{n, n}\left(U_{n+1}-U_{n}\right) \\
\frac{\partial}{\partial y} \Pi_{N} & =\beta-\frac{\partial^{2}}{\partial y^{2}} U_{N}-F_{N-1, N}\left(U_{N-1}-U_{N}\right)+\beta_{B}
\end{aligned}
$$

and $\beta_{B}$ is the bottom topographic $\beta$ parameter, $f_{0} \frac{\partial}{\partial y} \eta_{B} / H_{N}$.

A finite difference method has been used to solve this linear eigenvalue problem. Discretization renders the problem a matrix equation, which can be solved directly for temporal instability by standard procedures. I have developed a code based on a method introduced by Lin and Pierrehumbert (1993), the saddle point finder (see Appendix A), to solve the dispersion equation in the complex $(\omega, k)$ plane for pulse instability. Solving for spatial instability requires mapping of dispersion relation on complex $k$ plane, and the method is described in Appendix B.

In all the models we will use, the channel width is fixed at $320 \mathrm{~km}$ and resolution $10 \mathrm{~km}$ except where otherwise specified. The effects of resolution and channel width are tested in Appendix C. 


\subsection{Basic Flow and Parameter Calibration in Mul- tilayer Models}

The same set of CTD data mentioned above (Hall and Fofonoff, 1993) has been used to calculate the density field, the stratification profiles, and the geostrophic velocity field. We then determine the depth of each layer in layer models based on potential density analysis. And after the depth of each layer in a layer model has been calibrated, the geostrophic background velocity is averaged over the depth of each layer. The layer-averaged velocity field is then interpolated into the grid points. We assume a zonal basic flow in our model.

In order to determine the depth of each layer in layer model appropriately, we first look at potential density field. Figure 3.1 shows the contours of potential density field across the stream at $68^{\circ} \mathrm{W}$. The contours pass the center of the jet $\left(38^{\circ} \mathrm{N}\right)$ at depths of $500,700,1100$ meters, etc, which are labeled in the figure for convenient identification. The interval is $400 \mathrm{~m}$ in the interior and $200 \mathrm{~m}$ near the top and the bottom. From the figure, we can see that the thickness between contours increases towards North in the interior and decreases near the top and bottom. To see this feature more clearly, we calculate a spread ratio between every two contours. The spread ratio is defined as

$$
S=\frac{h_{1}-h_{2}}{\bar{h}}
$$

The thicknesses $h_{1}$ and $h_{2}$ are measured at $90 \mathrm{~km}$ and $80 \mathrm{~km}$ away from the jet center respectively (indicated by the dotted lines in Figure 3.1), where isopycnals start to level off. $\bar{h}$ is the thickness between the two contours at the jet center. The spread ratio is the percentage change in the thickness between the two contours and it captures the part of the potential vorticity gradient purely due to the depth change.

In Figure $3.2,{ }^{*}{ }^{*}$ indicates the spread ratios for layers between each pair of potential density contours. The solid line separates the positive spread ratios from 


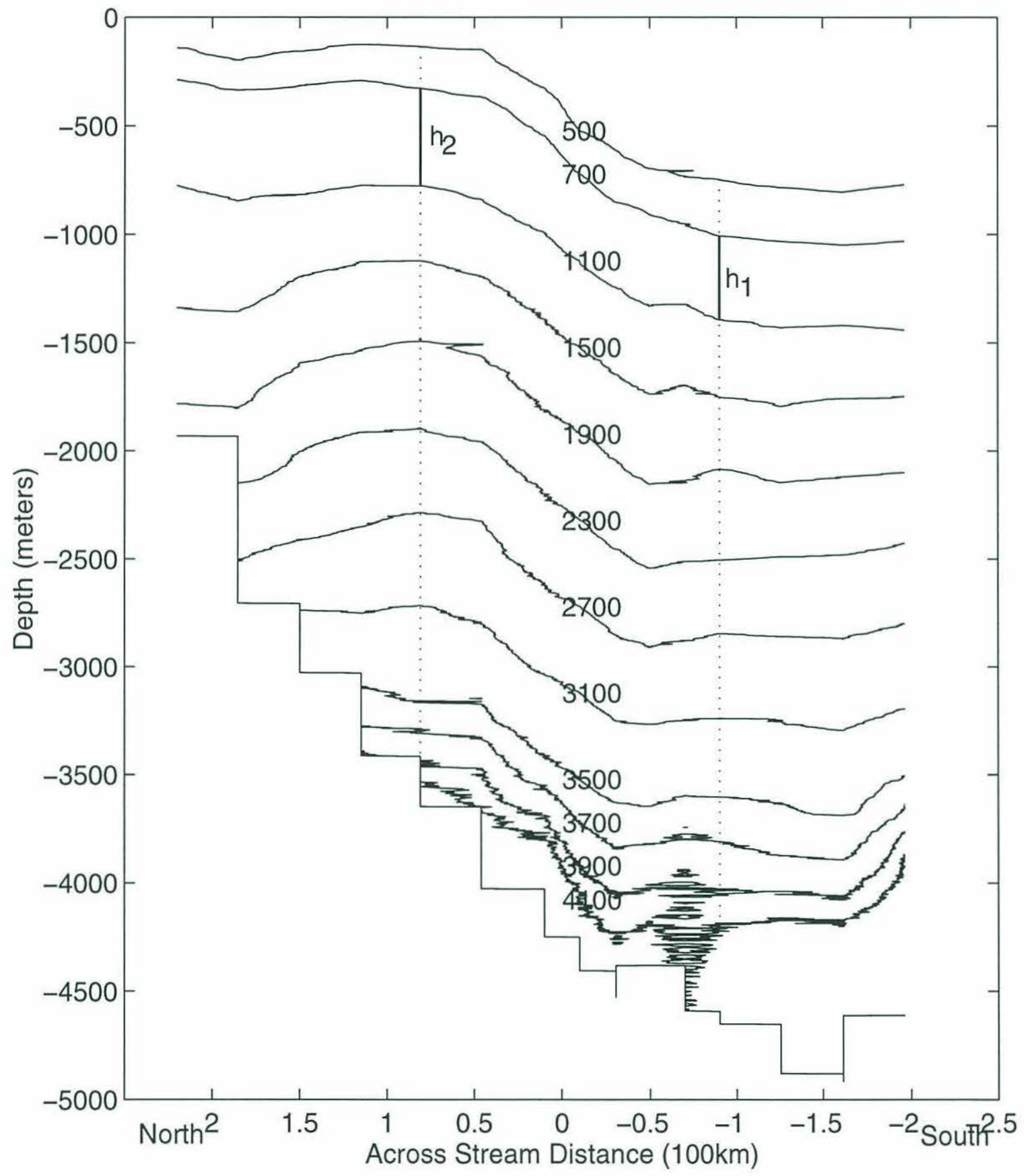

Figure 3.1: Potential density contours across the stream. 
negative ones. In general, the spread ratios are negative in the interior except two layers with slightly positive values. The layers with the largest spread ratios appear near the surface (above $700 \mathrm{~m}$ ) and the bottom (below $3500 \mathrm{~m}$ ). This suggests that we need an interface to separate the top layer and another one to separate the bottom layer from the interior if we want to capture the dynamics of the Gulf Stream properly.

If we use two-layer model to represent the Gulf Stream and put the only interface at the thermocline, the effect of topography will penetrate through the whole bottom layer. To see this more clearly, we calculate the spread ratio in the lower layer between thermocline and the bottom. The spread ratio in the lower layer is positive $(9.5 \mathrm{~m} / 100 \mathrm{~m})$ as indicated by dashed line in Figure 3.3, while the average spread ratio in the interior is negative $(-8.4 \mathrm{~m} / 100 \mathrm{~m}$, dotted line; the length of the line covers the depth range of averaging). Hence the spread ratio in the lower layer bounded by the thermocline and the topography does not reflect the real situation and using a twolayer model in the context may alter the characteristics of the instability concerned. If we use flat bottom instead of the real topography, the spread ratio $(25.0 \mathrm{~m} / 100 \mathrm{~m}$, dash-dotted line) in the bottom layer triples the average spread ratio in the interior. So neither form of the two-layer model will take the topographic effect into account properly. In Chapter 5, I will use a slope which is less than the true value to capture interior spread ratio better, but this model still cannot represent the change in sign occurring below 3500 meter.

Since the CTD data I used was only a snapshot of the Gulf Stream, I have included in Figure 3.4 a temperature contour plot from Leaman, et al (1989). The contours represent a multiyear average of temperature, obtained using PEGASUS current profilers off Cape Hatteras. Estimated directly from plot, the thickness between $3^{\circ} \mathrm{C}$ and $5^{\circ} \mathrm{C}$ changes from $1570 \mathrm{~m}$ at the Southern edge to $1900 \mathrm{~m}$ at the Northern edge, leading to an interior spread ratio of $(-9.5 \mathrm{~m} / 100 \mathrm{~m})$. This is comparable to the interior spread ratio $(-8.4 \mathrm{~m} / 100 \mathrm{~m})$ from the CTD section I used. If we use a two-layer 
model to represent the system and put the only interface in thermocline, for example, along $10^{\circ} \mathrm{C}$ contour, the spread ratio below the interface will be positive $(10 \mathrm{~m} / 100 \mathrm{~m})$ because of the large bottom slope. Hence a minimum of three layers is also necessary in this case.

We will use real topography with a little modification because our model cannot deal with the intersection of topography with a layer interface. In Figure 3.5, 'o' represents where CTD stopped, typically a few meters above the bottom, '*' the topography we have chosen to use. At about $50 \mathrm{~km}$ south of the jet, we have modified the topography slightly to smooth out a small scale bump which is very unlikely to affect large scale dynamics. At the southern edge, the topography is made flat. This is expected not to affect the dynamics significantly and the position of the station is actually beyond our model domain ( $320 \mathrm{~km}$ in width). The dashed curve (overlapped in part by solid curve) is the topography we actually used in all experiments when the 'real topography' is claimed, and has constant slope beyond $87 \mathrm{~km}$ from the center of jet. The difference in using such topography and the topography indicated by the solid curve is demonstrated in Figure 3.6, where solid curves represent the result corresponding to the topography identified by solid curve in Figure 3.5 and dashed curves to that identified by dark dashed curve Figure 3.5. In the experiment, I used a three-layer model with lowest interface at $3000 \mathrm{~m}$. The differences are almost invisible, suggesting that the effect of topography far away from the jet is small.

In all the models, the first interface is set at 660 meters at the core of the stream, and the lowest one at 3500 meters except for the two-layer model, as suggested by our potential density analysis. The six-layer model has three additional interfaces at 1370,2080, and 2790 meters so that the thickness between the first and the lowest interfaces are equally divided. We have chosen the additional interface in the fourlayer model at 1800 meters so that the model best matches the results of the six-layer model. In the five-layer model, the two additional interfaces are at 1370 and 2370 


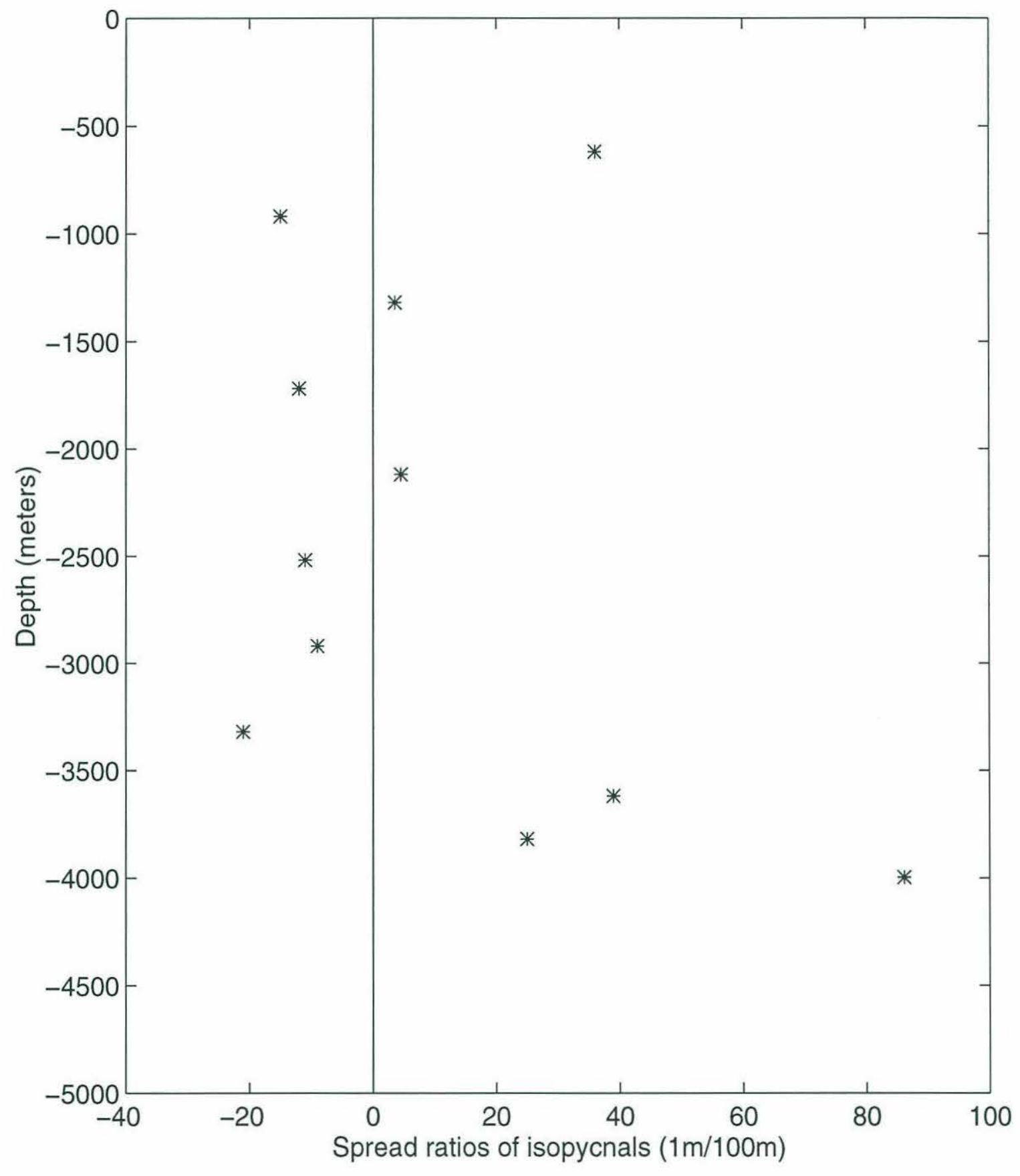

Figure 3.2: Spread ratios of isopycnals $(1 \mathrm{~m} / 100 \mathrm{~m})$. 


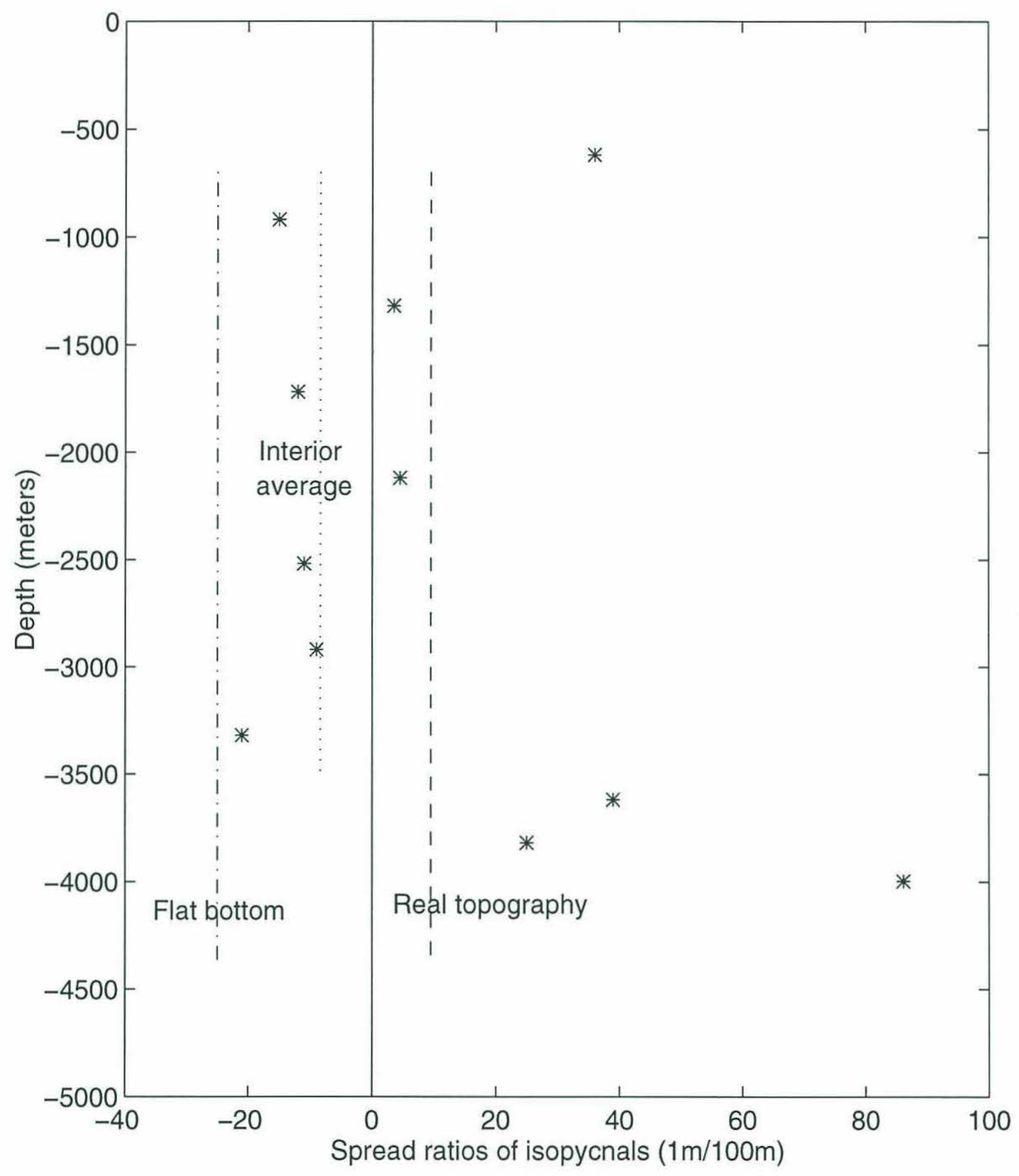

Figure 3.3: Spread ratios of isopycnals $(1 \mathrm{~m} / 100 \mathrm{~m})$. The dotted line is the average value in the interior, dashed the average value under thermocline when real topography is used in a two-layer model, and dash-dotted the average value under thermocline if the bottom were flat. 


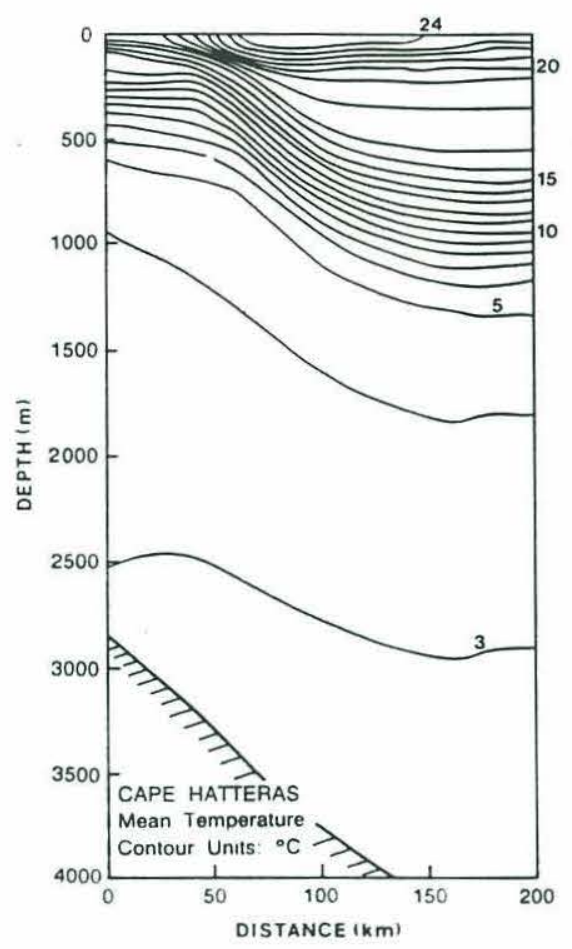

Figure 3.4: Contours of average temperature off Cape Hatteras. From Leaman, et al (1989).

meters. In all models, the topography is the modified "real topography" defined in the previous paragraph.

After we have determined the depth of each layer in multilayer models, we then take average of the geostrophic velocity over the depth of each layer. Taking the six-layer model as an example, we show the layer-averaged velocity structure in Figure 3.7 (narrow lines). There are six velocity profiles corresponding to six layers in the model. The maximum velocity in each layer decreases with the depth of the layer. We then fit the velocity structure in each layer with Gaussian type profile. The magnitude and width of the jet in each layer are determined by the magnitude and transport of the unsmoothed jet in the layer. The fitted profiles are also shown in Figure 3.7 (dark lines). 


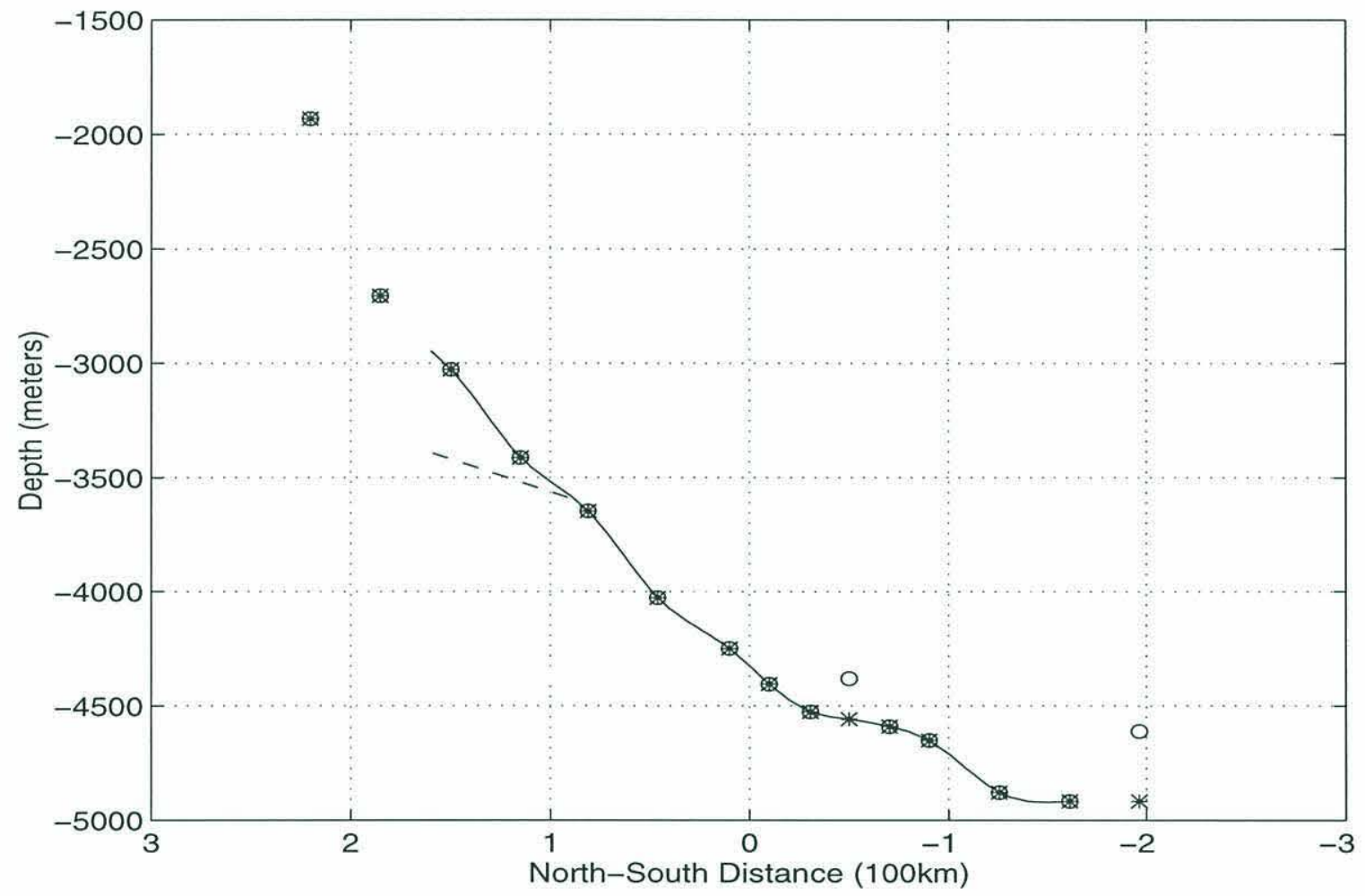

Figure 3.5: 'o' represents where CTD stopped near the bottom, '*' the topography we have chosen to use. The solid curve is interpolation of '*'s on model grids. The dashed curve (overlapped in part by solid curve) is the topography we actually used in all experiments. 

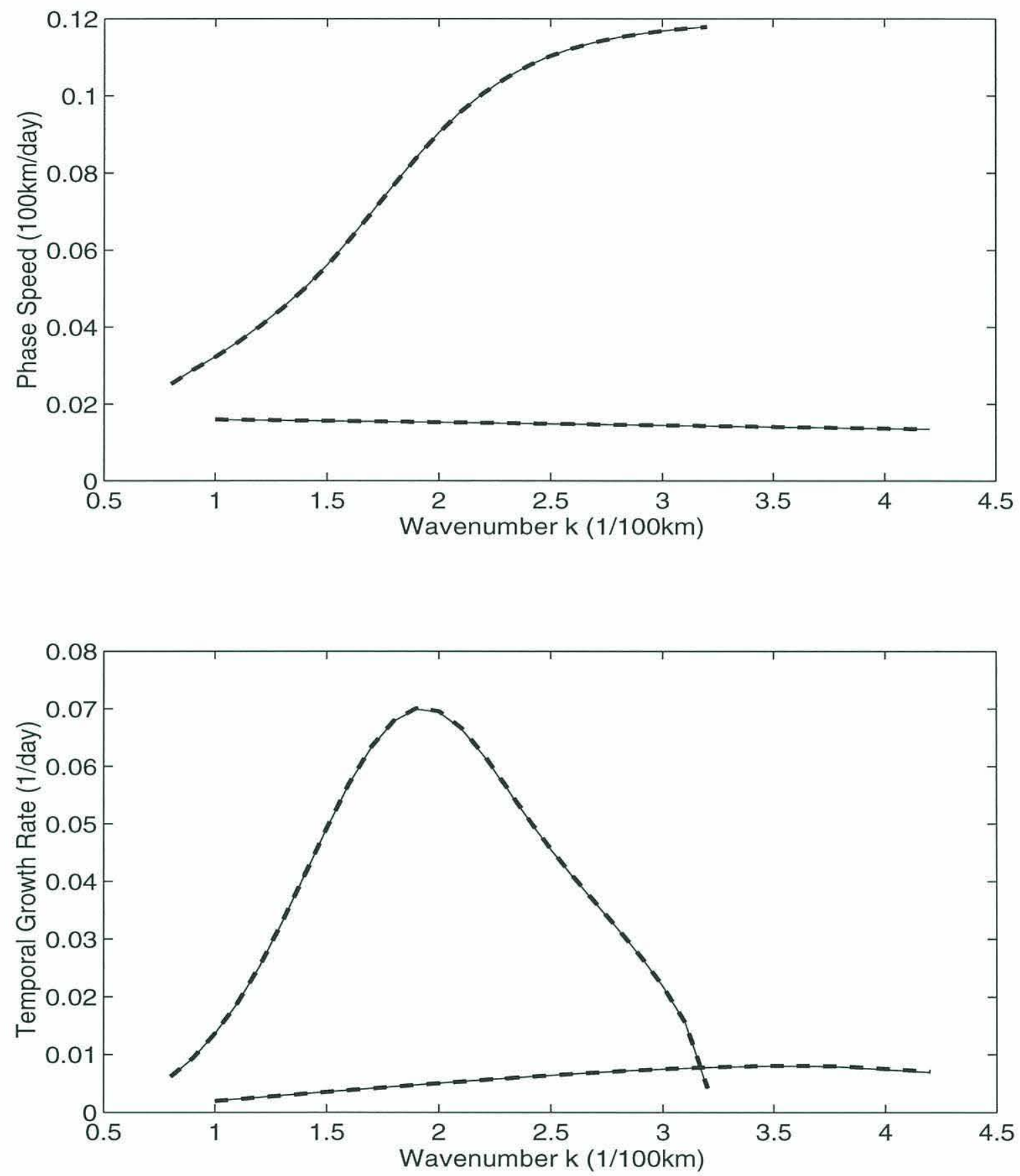

Figure 3.6: Solid curves represent the result corresponding to the topography identified by solid curve in Figure 3.5 and dark dashed curves to that identified by dashed curve in Figure 3.5. 


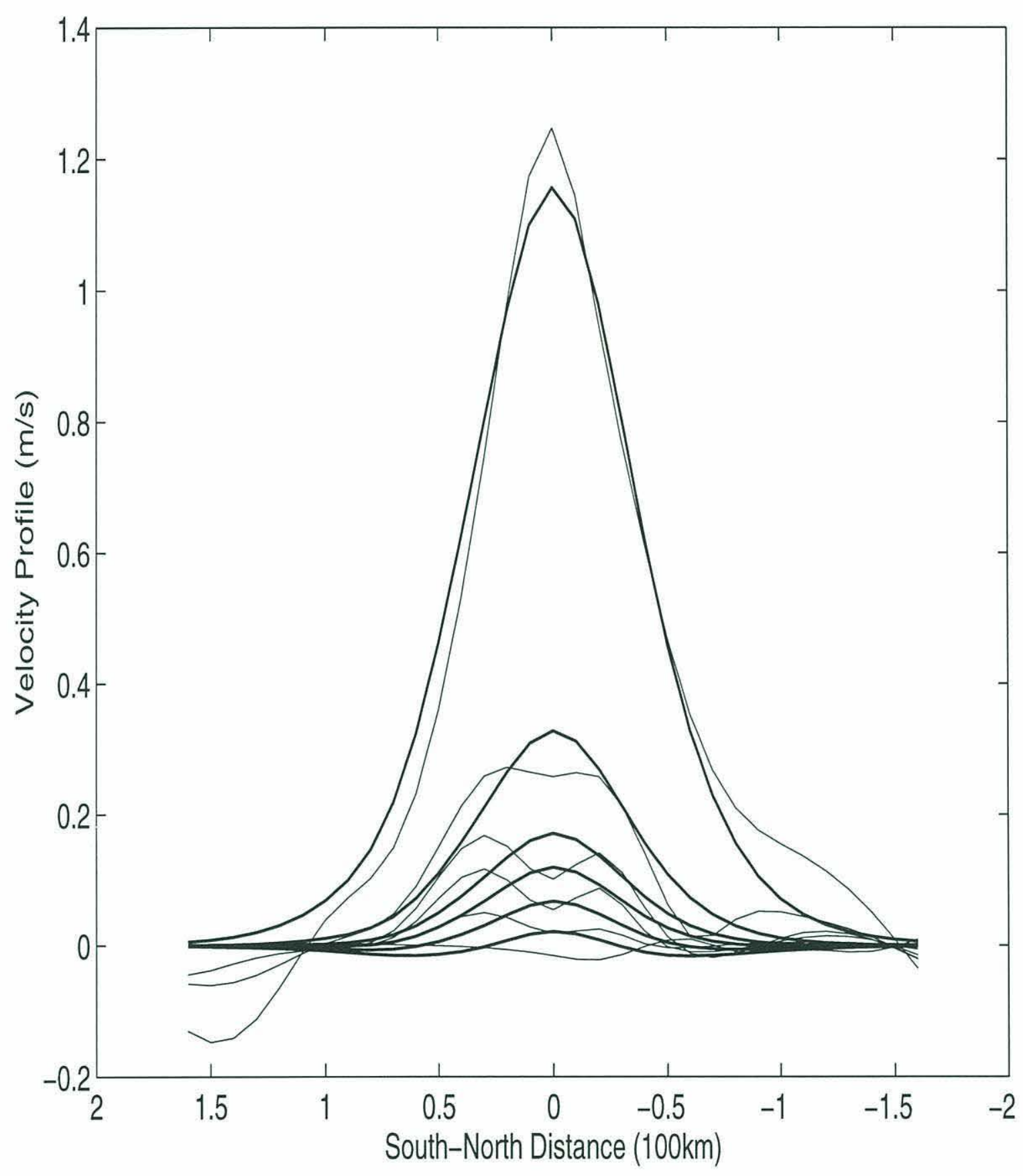

Figure 3.7: Velocity profiles in each layer of a six-layer model, the maximum velocity in each layer decrease with the depth of the layer. Narrow and dark curves: velocity profiles before and after fitting, respectively. 


\subsection{Comparison Among Multilayer Models}

Figure 3.8 shows the results of temporal instabilities corresponding to models with different number of layers. The upper panel shows phase speeds vs wavenumber, while the lower one shows temporal growth rate. For clarity, only the main unstable mode in each model is plotted and labeled with its corresponding number of layers.

As we can see in Figure 3.8, as the number of layers increases, the results tend to converge. In particular, the wavenumbers of the most stable waves are comparable except that in the two-layer model. The two-layer model gives a very different result in terms of the shape of the curve, maximum growth rate and wavenumber of the most unstable wave. The agreement among other models is worst on short wave side of the most unstable waves. In addition, when we plot more modes for four to sixlayer model in Figure 3.9, we see the curves on short wave side have not converged as much as on long wave side when the number of layers increases.

Figure 3.10 is the pulse instability version of the comparison. The ' $\mathrm{x}$ ' mark on each curve indicates the value at the peak of the envelope of a developed disturbance. Again we see the similar pattern that when the number of layer increases: the results tend to converge. However, some features here are more sensitive to the number of layers than in the case of temporal instability. Besides the ill-behaved two-layer model, the three-layer model has the opposite trend in term of wavenumber or frequency as a function of group velocity and the curve of the growth rate does not compare well with the four to six-layer models. This demonstrates that pulse instability is more sensitive to vertical resolution than temporal instability. This is probably because we need to use the group velocity as a variable in the pulse instability instead of wavenumber. Since a group velocity depends on the derivative of phase speed with respect to wavenumber, any inaccuracy in phase speed or wavenumber will be amplified in group velocity. So when we study pulse instability, we need to 

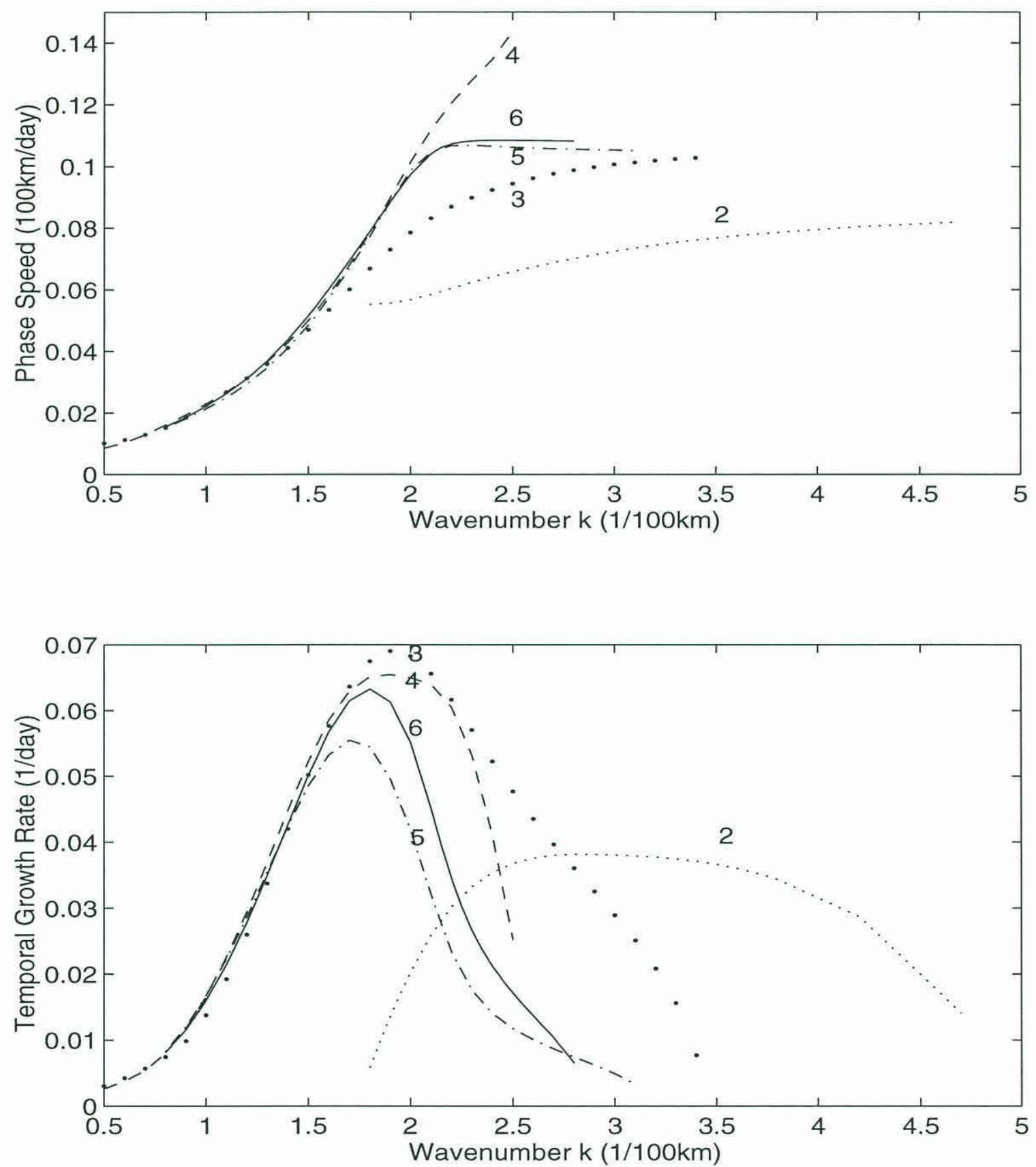

Figure 3.8: Temporal instabilities corresponding to models with different number of layers. Curves are labeled with their corresponding numbers of layers. 

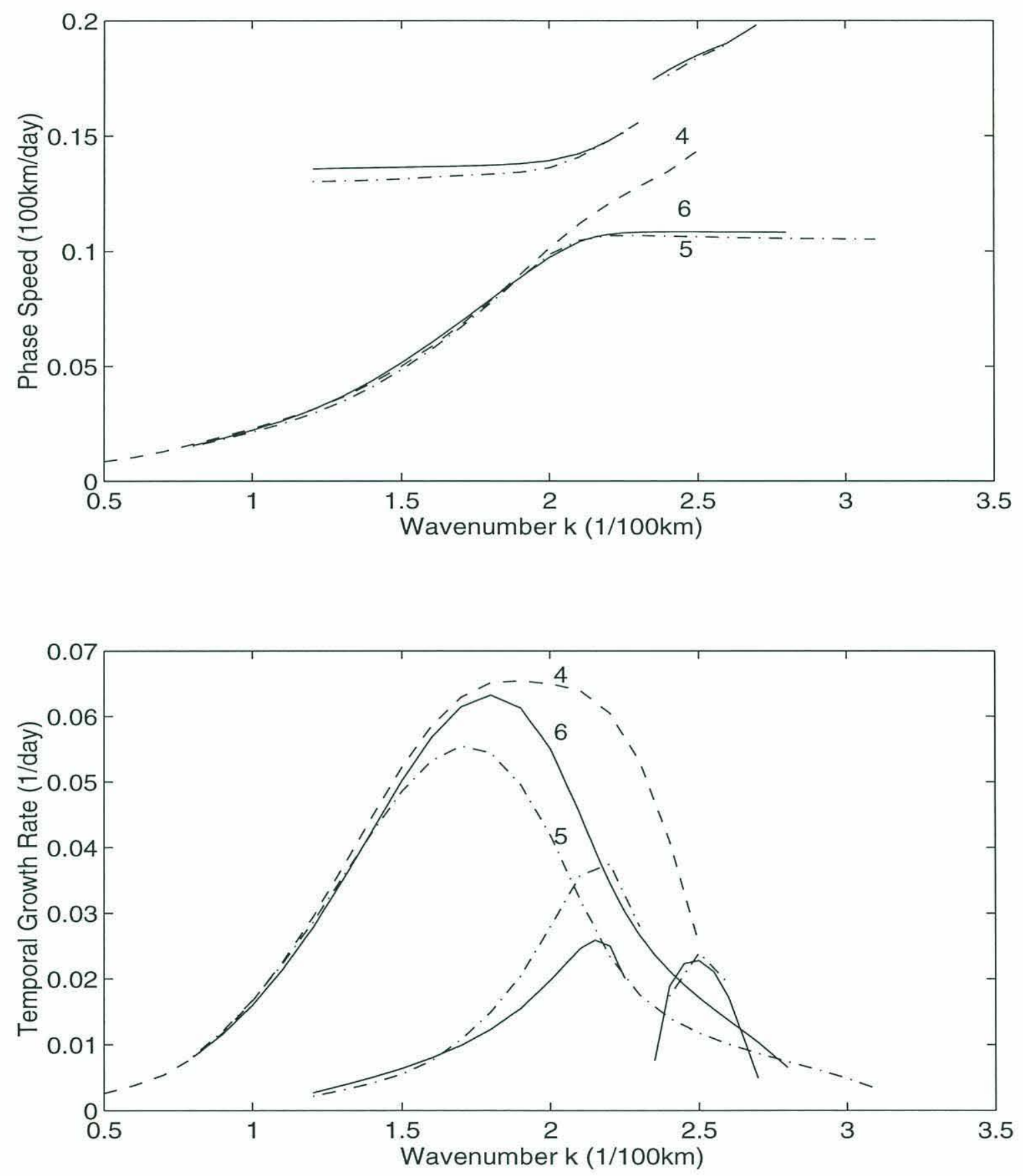

Figure 3.9: Temporal instabilities: comparison among four, five and six-layer models. Curves are labeled with their corresponding numbers of layers. 
use higher vertical and horizontal resolutions, especially near the surface and the core of the jet where the mean velocity changes rapidly.

From the above comparison, it appears that a six-layer model is appropriate in study of the Gulf Stream instability, especially for the main unstable mode. Hence we will focus on the analysis of a six-layer model in the next section.

\subsection{Temporal, Spatial and Pulse Instabilities in a Six-Layer Model}

Figure 3.11 redraws the three most unstable modes of temporal instability in the six-layer model. The most unstable wave (at the peak of the mode) has a wavelength of about $350 \mathrm{~km}$, a period of 44 days and a growth rate of $0.063 /$ day. The growth rate corresponds to an e-folding time of 16 days. These results will be compared with observational analysis by Lee and Cornillon (1996b) in Chapter 4 . The other two modes have growth rates of .026 and $.023 /$ day respectively, which are less than $50 \%$ of the first mode.

Figure 3.12 compares spatial growth rates (SGR) with equivalent spatial growth rates (ESGR) and pseudo-equivalent spatial growth rates (PESGR) for the three most unstable modes. The bumps on the SGR curve look peculiar and will be discussed later. The vertical lines indicate the real parts of the frequencies at the peaks of temporal unstable modes. The corresponding periods are also labeled. As I described in Chapter 2, SGR is calculated through dispersion equations with real frequency. Both ESGR and PESGR, estimates of SGR, are calculated from the temporal growth rates divided by group velocity and phase speed respectively.

For the most unstable mode, the maximum SGR exceeds by $32 \%$ ESGR predicted from the temporal instability at the most unstable wavenumber. Dominated 

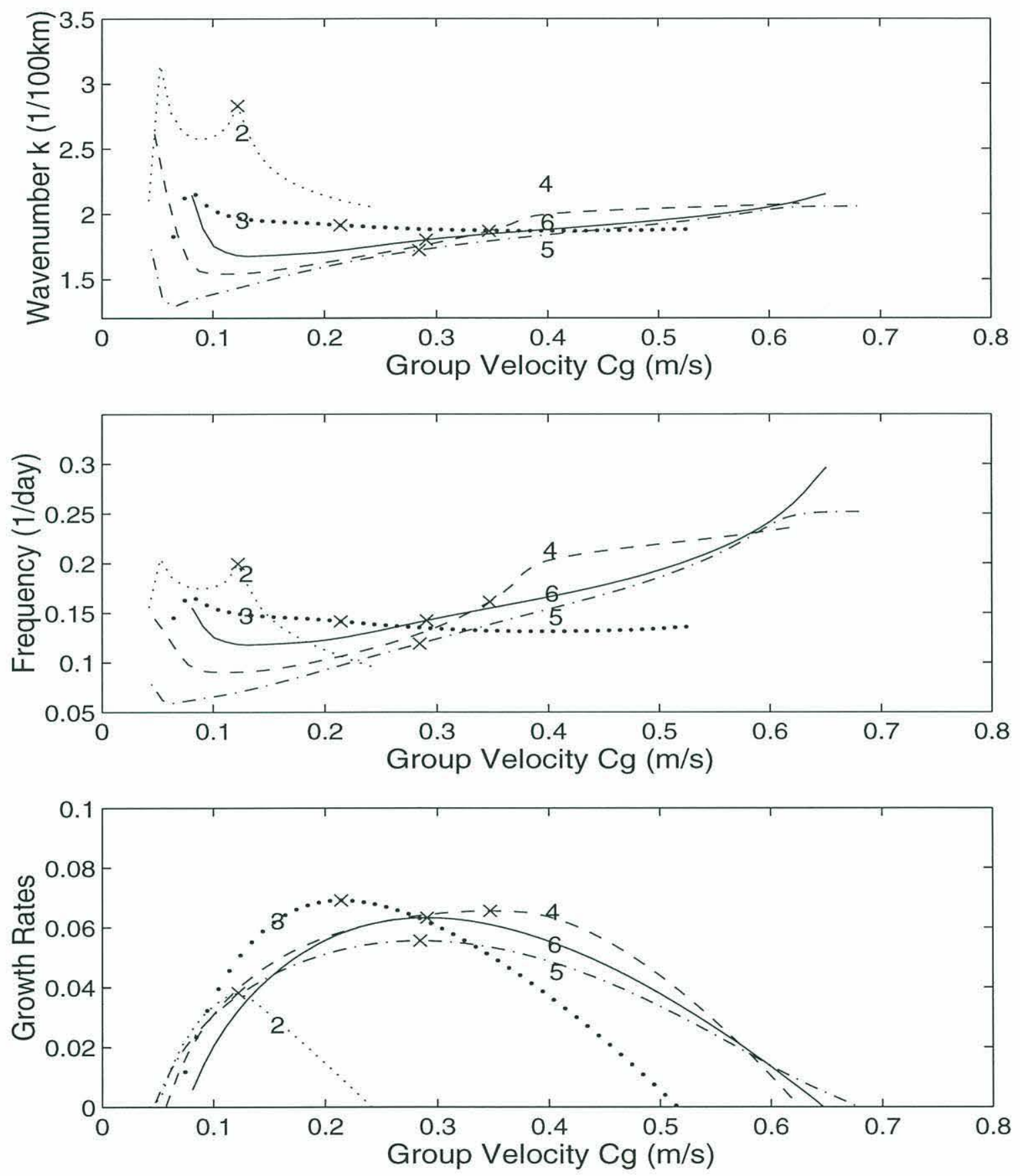

Figure 3.10: Pulse instabilities corresponding to models with different number of layers. Curves are labeled with their corresponding numbers of layers. 

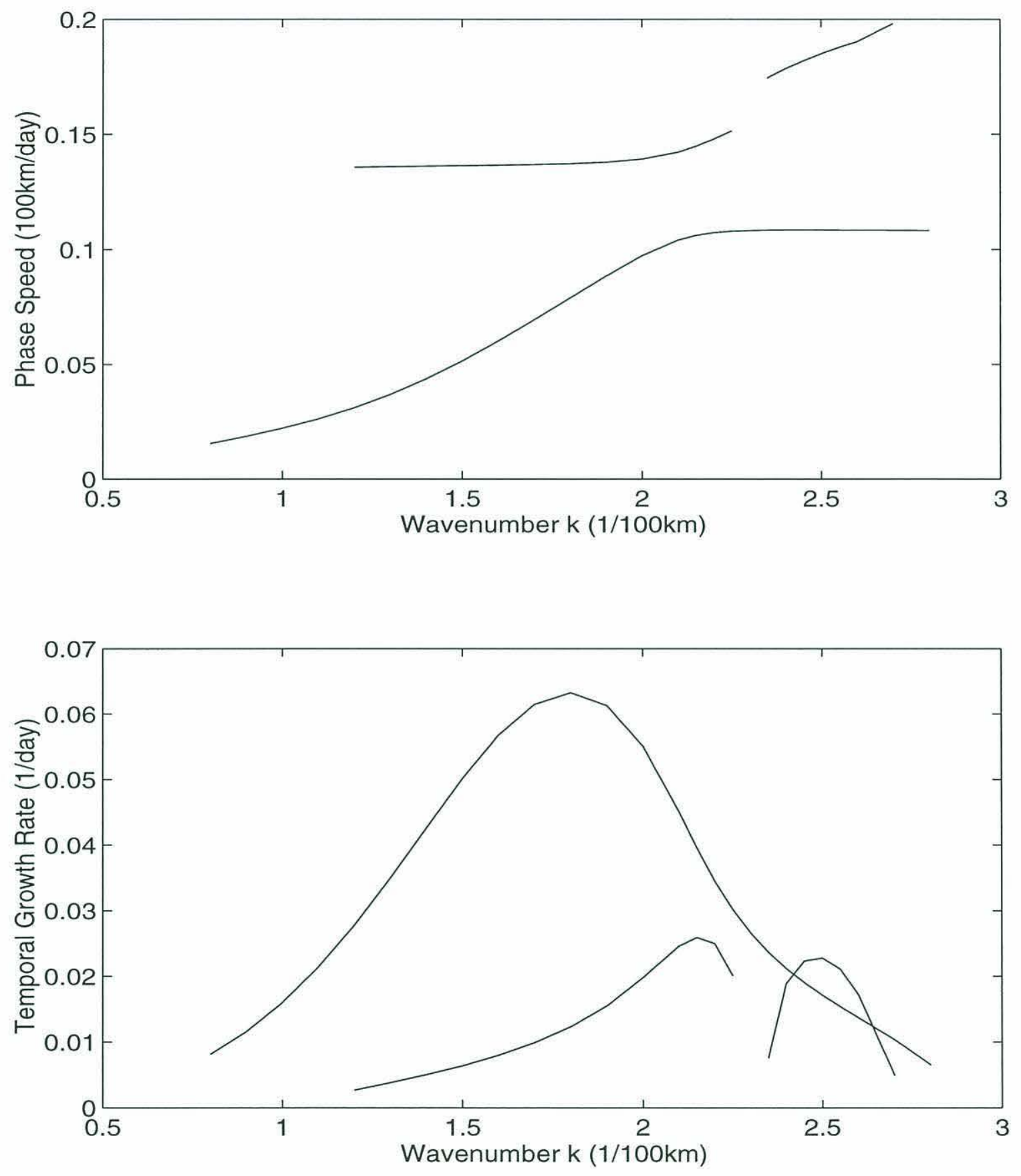

Figure 3.11: The three most unstable modes from temporal instability in the six-layer model. 

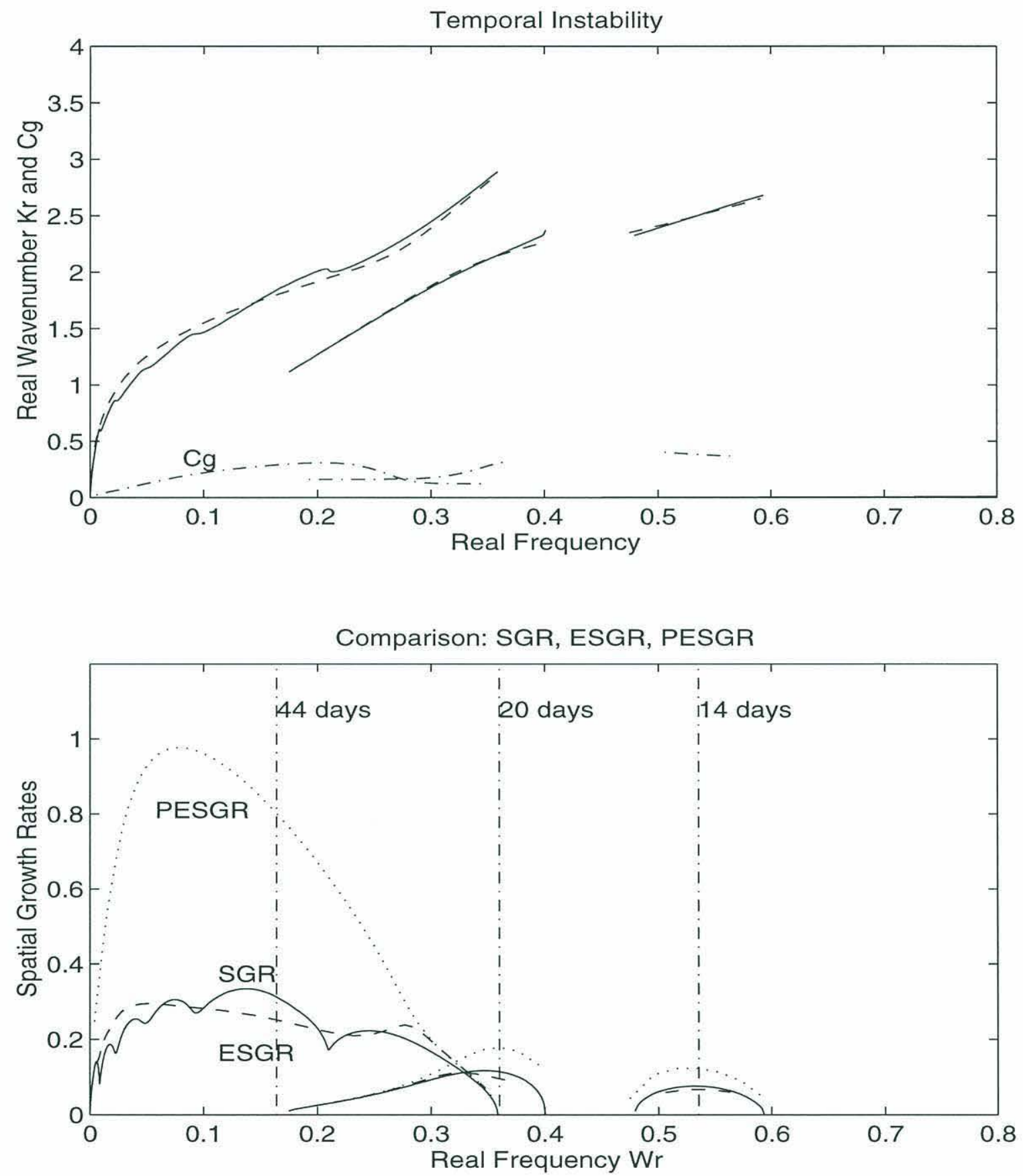

Figure 3.12: Comparison between temporal and spatial instabilities in the six-layer model. 
by the small group velocity in transformation, however, the ESGR curve peaks at $\omega=0.04$, which corresponds to a period of 160 days. The maximum SGR is at $\omega=0.14$ (52 days), while the most temporally unstable wave is at $\omega_{r}=0.164$ (44 days).

For all of the three modes, ESGRs are closer to SGRs than PESGRs are, especially at low frequency where PESGR almost triples SGR. This suggests that ESGR is a better estimate of SGR than PESGR,

The upper panel of Figure 3.12 shows group velocities and real wavenumbers of the three modes. Group velocities have been calculated from temporal instability. The solid curves are real wavenumbers calculated from spatial instability, while the dashed curves temporal instability. The wavenumbers from both instability theories are actually very close, especially right at the peaks.

I now return to discuss the strange bumps on the SGR curve. They are plausibly due to multiple roots merging together. In this model, there are 186 grid points. The model gives 186 roots of $\omega$ when $k$ is given and 558 roots of $k$ when $\omega$ is given. It is possible that some of these roots merge together to form a mode with such bumps. Figure 3.13 redraws the first mode of spatial instability (the solid curve with a larger maximum growth rate) from Figure 3.12 and an additional mode labeled as pinch-off mode. (The dashed curve will be explained in the next paragraph.) For convenience I call the first mode as a merger mode. Near $\omega_{r}=0.21$, it seems that the merger mode has be formed by connecting the upper parts of two roots which could have been separate for some parameter range, whereas the pinch-off mode has been formed by connecting the lower parts of the two roots.

These bumps in the first mode are very sensitive to the strength of the mean flow. To see this more clearly, I have added a uniform barotropic flow of $0.02 \mathrm{~m} / \mathrm{s}$ to the velocity profile shown in Figure 3.7, and compute the spatial growth rate with the same model. The dashed curve in Figure 3.13 shows the first mode of the spatial 


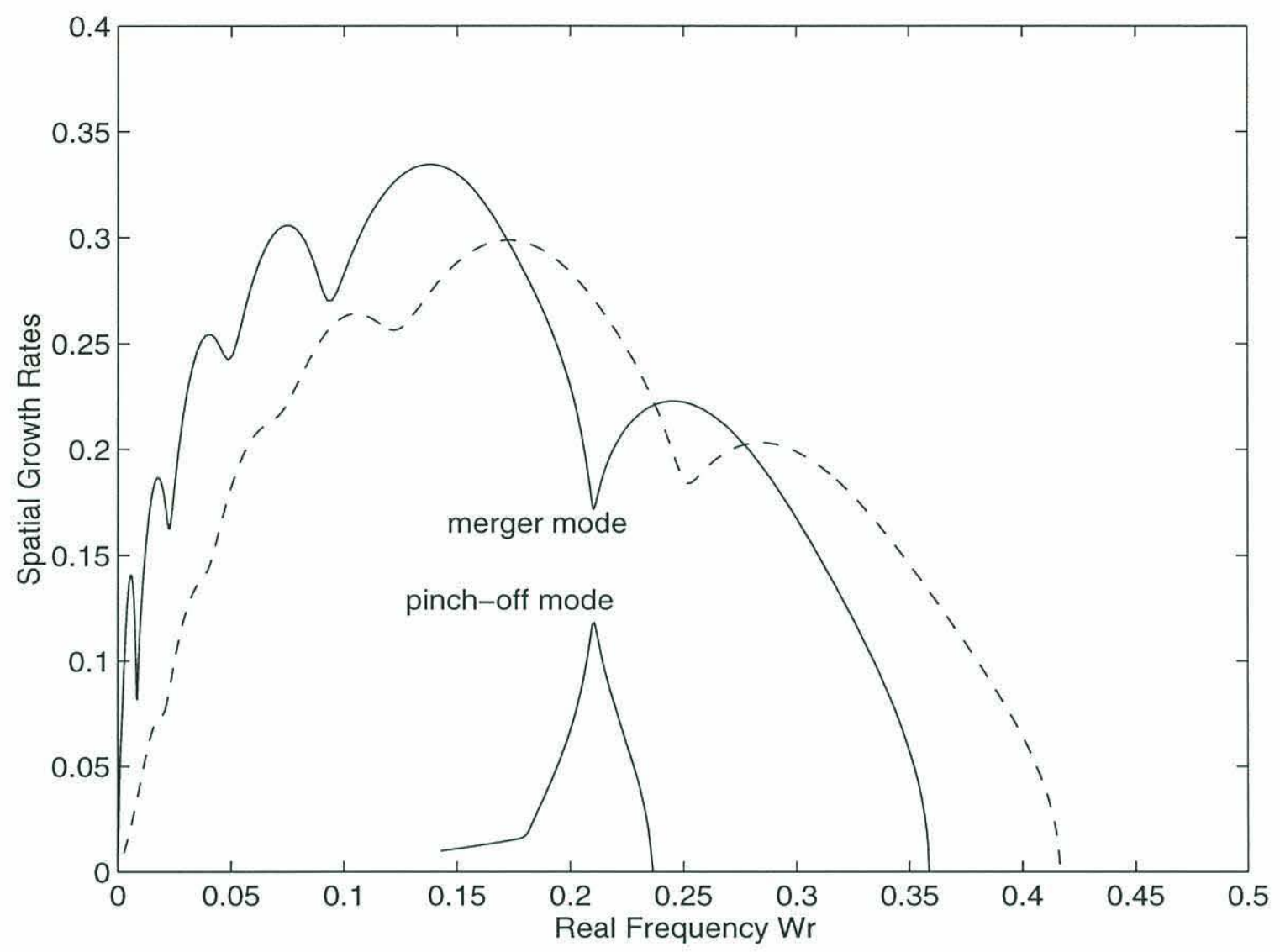

Figure 3.13: Comparison of spatial instabilities between the six-layer models with and without an additional uniform barotropic flow of $0.02 \mathrm{~m} / \mathrm{s}$. The solid curves represent the former case and dashed the latter.

instability. We see that the bumps in this case have been smoothed substantially by the weak additional barotropic flow. This is probably because the additional barotropic flow has changed the structure of the roots significantly so that the way they intersect, merge or overlap has become much smoother.

Figure 3.14 shows pulse instability for the three most unstable waves. The upper and lower panel display the growth rates of individual peaks and the envelopes respectively, as functions of the group velocity. The growth rate of an individual peak is calculated from Equation 2.3.13, which is

$$
\nu_{i p e a k}=\nu_{i}\left(C_{g}\right)+\left(c_{p h}-C_{g}\right) \frac{\partial \nu_{i}}{\partial C_{g}}
$$


The ' $x$ ' mark on each curve indicates the maximum of the envelope, whereas the dark ' $x$ ' mark represents where an individual peak grows fastest among all waves corresponding to the same mode.

From Figure 3.14, again we see the similar feature as in Phillips' model: the growth rate of an individual peak closely depends on its position relative to the peak of the envelope and can be very different from that of the envelope. The ratios of the growth rates of the fastest growing waves to the maximum growth rates of the envelopes are 2.39, 3.67 and 2.07 for the first three modes, whereas it is 1.78 in Phillips' model. So the feature is stronger in this Gulf Stream model.

Figure 3.15 shows the wavenumbers and frequencies of the first three modes. The ' $x$ ' and dark ' $x$ ' marks indicates the most unstable waves (the maximum of the envelope growth) and the fastest growing waves. Over a large range ( $C_{g}$ from .15 to $.60 \mathrm{~m} / \mathrm{s})$, the first and second modes have the same sloping trends of wavenumbers and frequencies, similar to the trends in Phillips' model, whereas the third mode has the opposite trends. Since the growth rate of first mode is dominant over the others, this model suggests that the fastest growing waves should have shorter and higher frequency than the most unstable wave in the ocean given the velocity profile.

Figure 3.16 and Figure 3.17 show how a peak in the leading edge of the envelope evolves according to the most unstable mode in Figure 3.14. The demonstration has been done in the Phillips' model, but it is important to use a more realistic model to see whether the conclusion from Phillips' model still holds. The method has been described in Section 2.3.5 and will be briefly repeated here for convenience. Suppose a disturbance is initiated at $x=0$ and $t=0$. After a significant amount of time, the wave envelope approaches its asymptotic form of the solution. If we focus on an individual peak in the leading edge of the disturbance envelope, we calculate the values of $C_{g}=x / t$ and can have $\nu_{i p e a k}$ and $\nu_{i}$ for that spot from Figure 3.14. We then compute the amplitude and phase speed of the peak for next time step. Repeating 

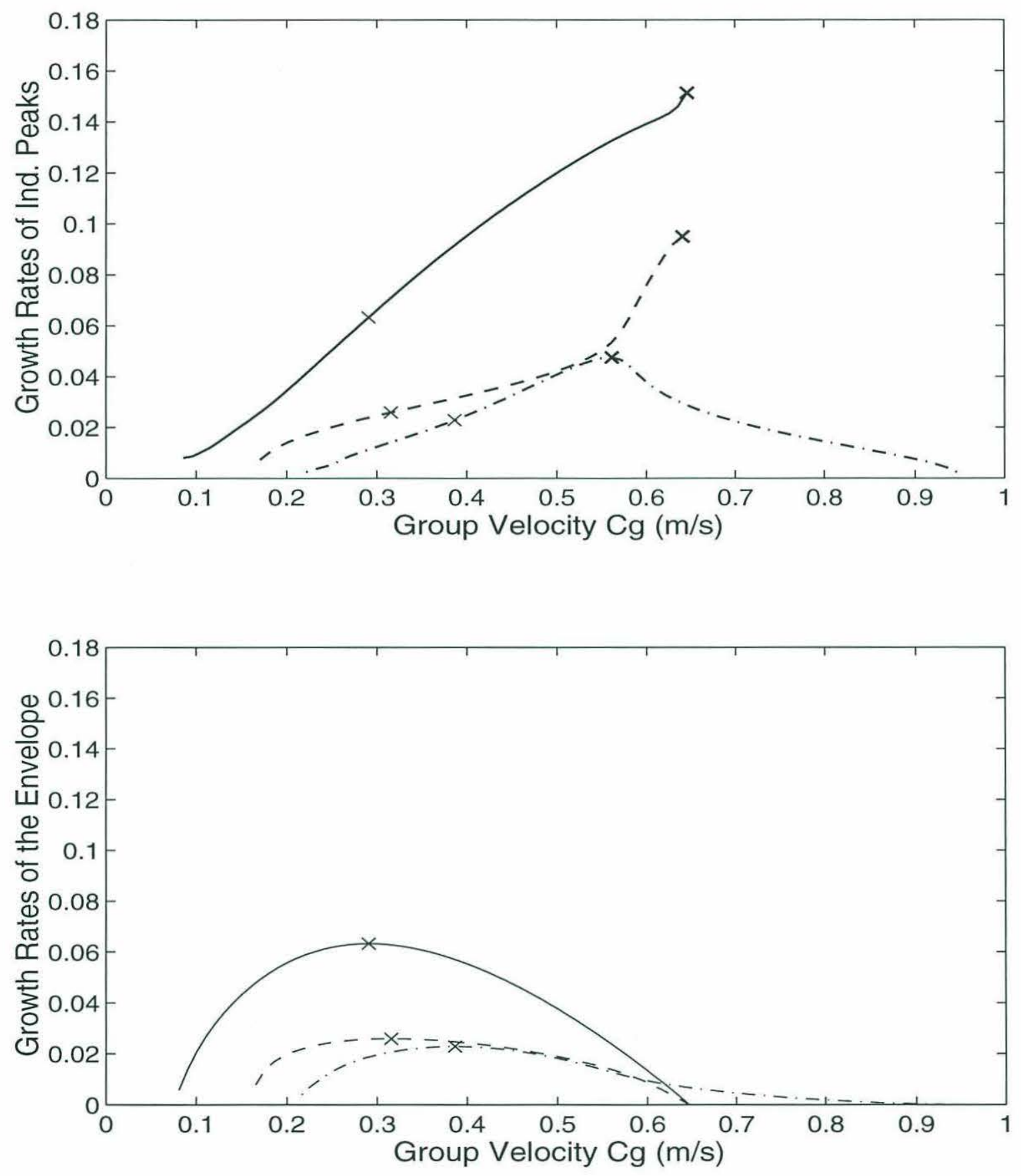

Figure 3.14: Pulse instability in the six-layer model. Solid, dashed and dash-dotted curves represent the three most unstable modes respectively. The upper and lower panel show the growth rates of individual peaks and the envelopes, respectively. 

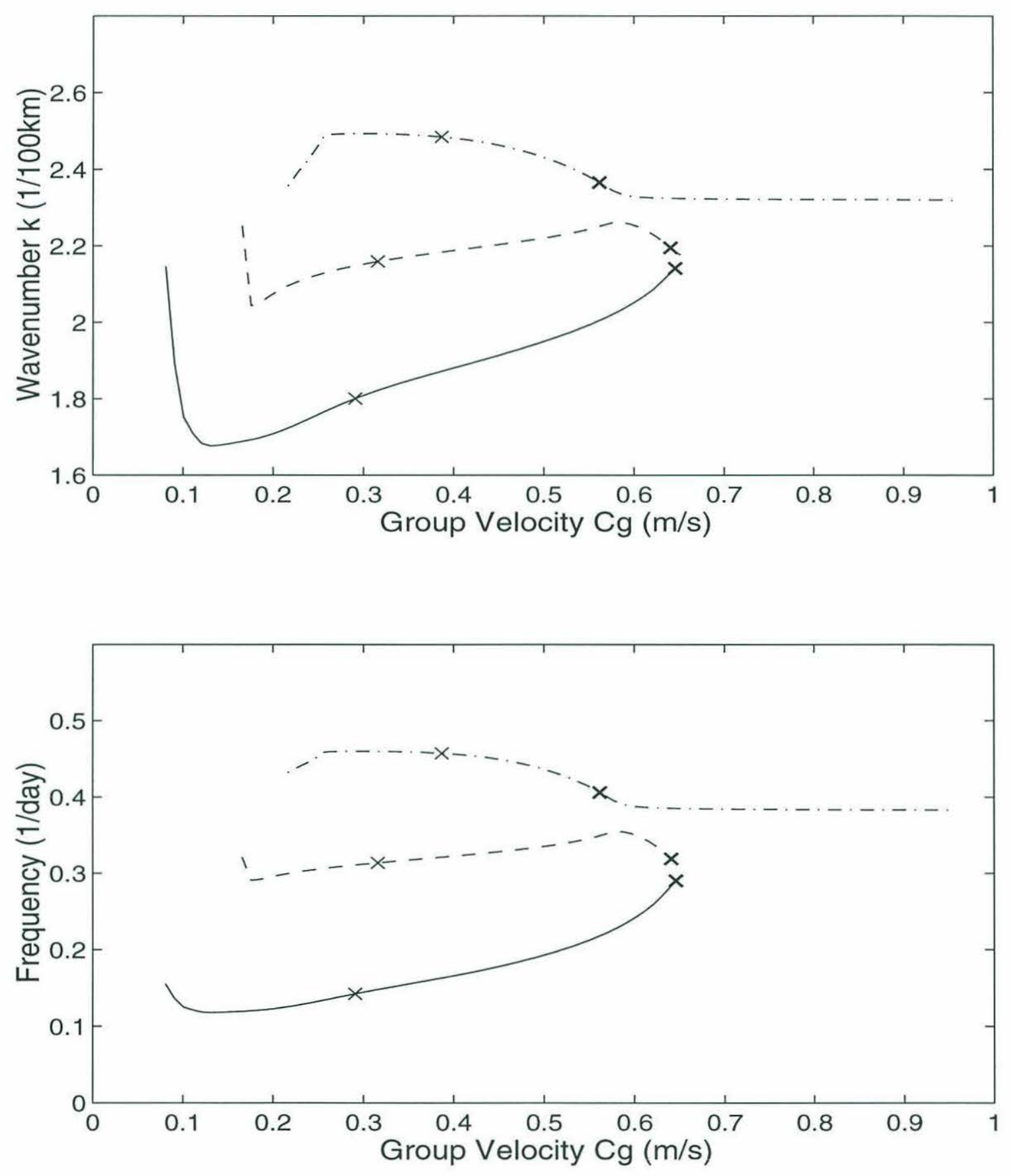

Figure 3.15: The upper and lower panel show the wavenumbers and frequencies, respectively. 
the procedure step by step, we collect the history of the individual peak. Figure 3.16 and Figure 3.17 show the results of such an experiment. In Figure 3.16, growth rate, phase speed and wavenumber are plotted vs time, while in Figure 3.17 vs log of amplitude (assuming initial amplitude of 1, or scaling the amplitude by its initial amplitude). What we can see from the both figures is that the growth rate, phase speed and wavenumber of the individual peak decrease as the peak evolves. When the peak is on the leading edge of the envelope, its growth rate can be much larger than that of maximum envelope growth. The pattern is very similar in Figure 3.17. The growth rate of the individual peak decreases as the amplitude increases, so does the wavenumber. So the features appeared in Phillips' model also appear here.

In the experiment, an individual peak in the leading edge grows as fast as $220 \%$ the rate of the envelope growth. And when it falls in trailing edge, it grows as slow as $30 \%$ the rate of the envelope growth. Thus a peak in the leading edge can grow several times as fast as another peak in the trailing edge. In Phillips' model, the fastest and slowest growing individual peaks have growth rates of $178 \%$ and $80 \%$ of the maximum envelope growth, respectively. So the difference between the waves in the leading edge and slowest growing wave behind the maximum of the envelope is much larger here than in Phillips' model, probably due to the jet structure which is not present in Phillips' model. Whether such a huge difference can be realized in the context of the Gulf Stream will be addressed in Chapter 5, where I will use a numerical model with relatively realistic features of the Stream.

\subsection{Summary and Conclusions}

From the analysis of potential density and spread ratio, we have shown that a minimum of three layers is necessary to address the effect of topography properly. Comparison among two to six-layer models support this point. The comparison also 

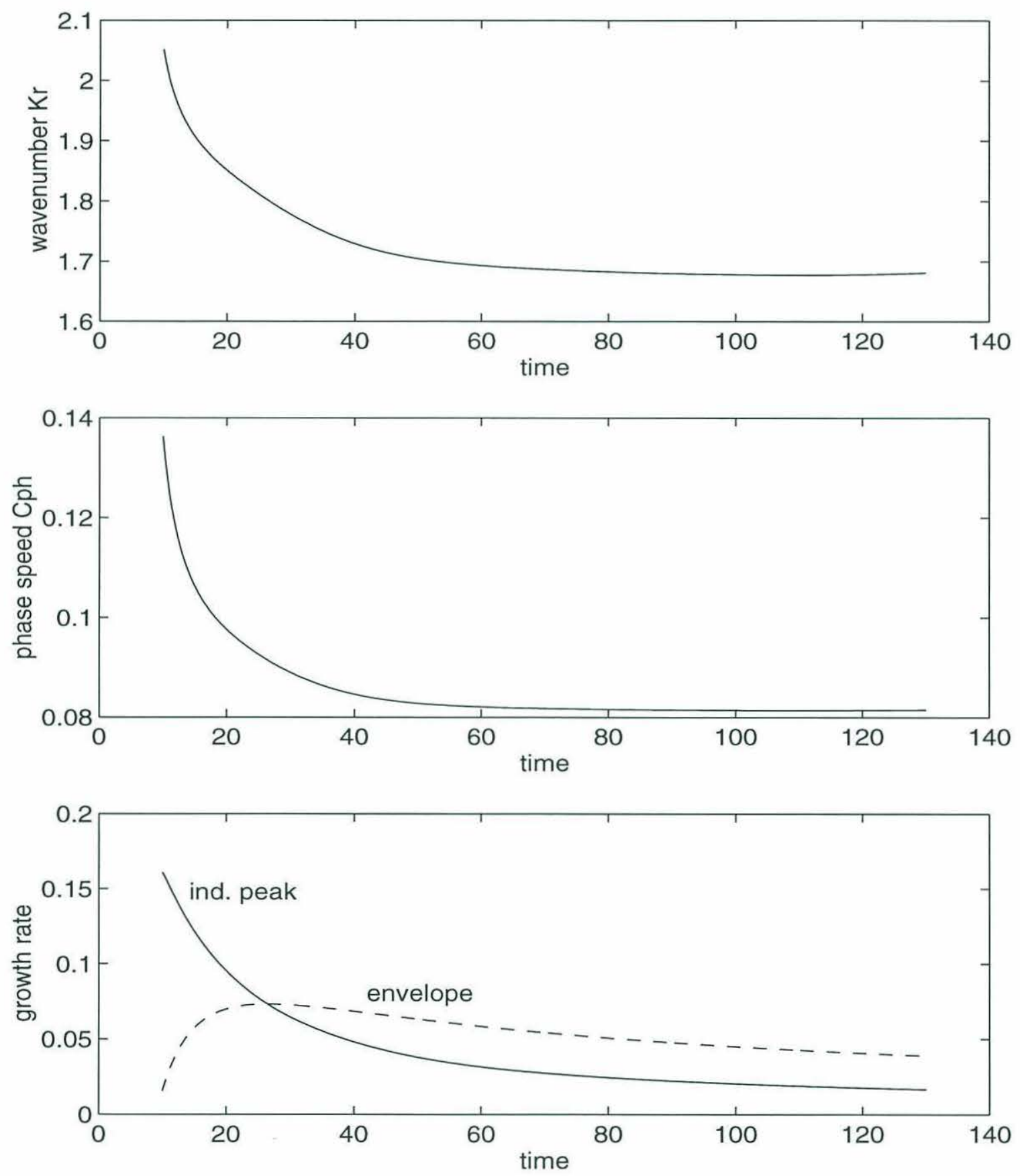

Figure 3.16: Properties of an individual peak vs time. 

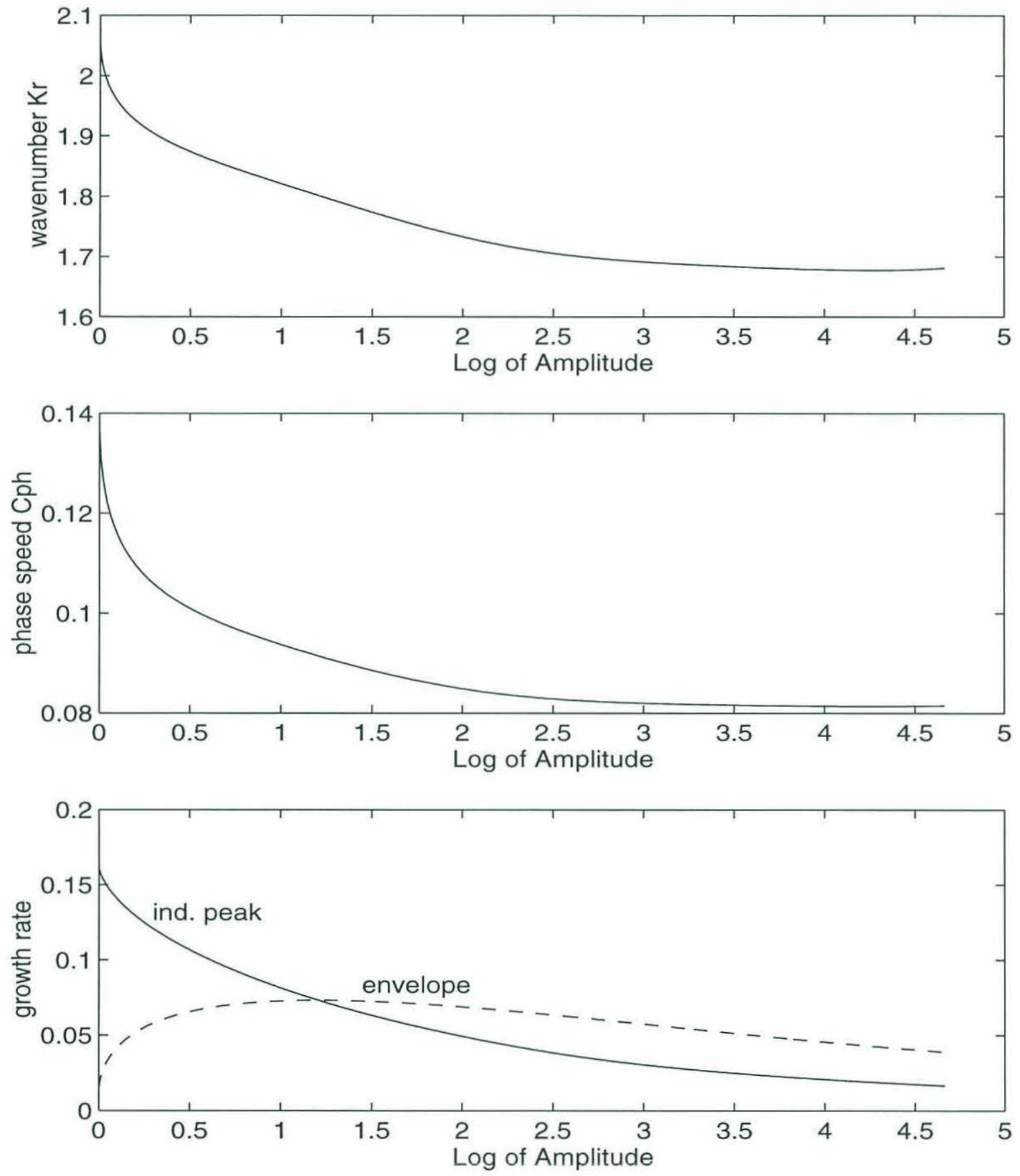

Figure 3.17: Properties of an individual peak vs log of amplitude. 
shows that short waves are more sensitive to vertical resolution. In the case of pulse instability, a minimum of five layers is necessary to capture the characteristics of the instability.

Spatial Growth Rate (SGR) is generally larger by about $30 \%$ than Equivalent Spatial Growth Rate (ESGR) converted from temporal instability. Pseudo-Spatial Growth Rate exceeds SGR by $200 \%$ at low frequency and is not a good estimate of SGR in general.

In the case of pulse instability, the growth rate of an individual peak can be very different from that of wave envelope. If the initial disturbance is a single pulse, the growth rates of individual peaks can vary from $30 \%$ to $220 \%$ of that of the peak of the wave envelope and cannot be predicted by temporal or spatial instability theories.

To avoid repetition, we will defer comparison of our results of the QG model to data until the next chapter, where a shallow water model will be studied and compared with the QG model as well as observations. 


\section{Chapter 4 \\ Shallow-Water Models}

\subsection{Motivation}

In Chapter 2 and 3, we have used QG models to study the characteristics of temporal, spatial and pulse instabilities. Since QG models can only handle well small Rossby number and small slopes of isopycnals and topography, we need to examine first whether these conditions have been violated in the Gulf Stream. From the observations, the Rossby number was estimated to be .3-.5 in the Gulf Stream east of Cape Hatteras(Bower, 1989). The depth of the top of the thermocline varies from around $800 \mathrm{~m}$ in Southern side to about $200 \mathrm{~m}$ in Northern side, and the depth of the bottom changes from about $4900 \mathrm{~m}$ to $1900 \mathrm{~m}$ across a CTD section at $68^{\circ} \mathrm{W}$ running from $36.3^{\circ} \mathrm{N}$ to $40^{\circ} \mathrm{N}$ (Hall and Fofonoff, 1993). Such large changes in the depths of isopycnals and topography may violate the assumptions of QG theory. Therefore one may expect that the QG approximation is not accurate in the context of the Gulf Stream, and a question naturally arises whether the results obtained in QG models can be extended to the primitive equation model or shallow-water model. This chapter is devoted to the comparison between QG and Shallow-Water models $(\mathrm{SW})$. 


\subsection{Model Description}

In this chapter, I will use a six-layer channel model. The model, however, has been developed for $\mathrm{N}$ layers as long as there is no outcropping or intersection of interfaces with topography. The model configuration and topography are the same as in the QG six-layer model used in the last chapter. The channel width is $320 \mathrm{~km}$ and the boundary conditions assume no normal flow across the channel walls.

Let $n=1$ indicate the top layer, and $n=N$ the bottom one. The equations of motion can be written

$$
\begin{aligned}
& \frac{\partial}{\partial t} u_{n}+u_{n} \frac{\partial}{\partial x} u_{n}+v_{n} \frac{\partial}{\partial y} u_{n}-f v_{n}=-\sum_{i=1}^{n} g_{i} \frac{\partial}{\partial x} \eta_{i} \\
& \frac{\partial}{\partial t} v_{n}+u_{n} \frac{\partial}{\partial x} v_{n}+v_{n} \frac{\partial}{\partial y} v_{n}+f u_{n}=-\sum_{i=1}^{n} g_{i} \frac{\partial}{\partial y} \eta_{i}
\end{aligned}
$$

where $u_{n}, v_{n}$, and $\eta_{n}$ are along stream, across stream velocities, and elevations of the surface and the interface. The reduced gravity factors are

$$
g_{n}=g \frac{\rho_{n}-\rho_{n-1}}{\rho_{n}}
$$

and $\rho_{0}$ is 0 by definition.

The equations of continuity are

$$
\frac{\partial}{\partial t} h_{n}+\frac{\partial}{\partial x}\left(h_{n} u_{n}\right)+\frac{\partial}{\partial y}\left(h_{n} v_{n}\right)=0
$$

with $h_{n}=H_{n}+\eta_{n}-\eta_{n+1}$. When $n=N, \eta_{N+1}=\bar{\eta}_{N+1}=h_{b}$, the elevation of the bottom. The lateral boundary conditions are chosen to be

$$
v_{n}=0, \text { at } y=0, L_{y}
$$

where $L_{y}$ is the width of the domain. We consider the case where the basic flow only has an along-stream component (x-direction) and we separate physical quantities into 
basic state fields and perturbation components,

$$
\begin{aligned}
& u_{n}=\bar{U}_{n}(y)+u \prime_{n}(y) \\
& v_{n}=v \prime_{n}(y) \\
& \eta_{n}=\bar{\eta}_{n}(y)+\eta I_{n}(y) \\
& h_{n}=\bar{h}_{n}(y)+h \prime_{n}(y)=H_{n}+\left(\bar{\eta}_{n}-\bar{\eta}_{n+1}\right)+\left(\eta \prime_{n}-\eta \prime_{n+1}\right)
\end{aligned}
$$

where $\eta^{\prime}{ }_{N+1}$ is defined to be $0 . H_{n}$ is constant and represents the mean thickness of n-th layer.

We assume the mean field is in geostrophic balance. Given the basic state flow $\bar{U}_{n}$, the displacement of interfaces in the basic state can be determined as

$$
\bar{\eta}_{n}=\eta_{n 0}-\frac{1}{g_{n}} \int\left(\bar{U}_{n}-\bar{U}_{n-1}\right) f d y
$$

where $\eta_{n 0}$ is an integration constant and $\bar{U}_{0}$ is defined to be 0 .

We now substitute Equations (4.2.4a-d) into Equations (4.2.1a-b) and Equation (4.2.2). If we study only the linear instability problem, we can neglect nonlinear terms in the resulting equations. For simplicity, we drop all primes and have

$$
\begin{aligned}
& u_{n t}+\bar{U}_{n} u_{n x}+\bar{U}_{n y} v_{n}-f v_{n}+\sum_{i=1}^{n} g_{i} \eta_{i x}=0 \\
& v_{n t} \quad+\bar{U}_{n} v_{n x}+f u_{n}+\sum_{i=1}^{n} g_{i} \eta_{i y}=0 \\
& \eta_{n t} \quad+\sum_{i=n}^{N}\left\{\left(\bar{H}_{i}+\bar{\eta}_{i}-\bar{\eta}_{i+1}\right)\left(u_{i x}+v_{i y}\right)+\left(\bar{\eta}_{i y}-\bar{\eta}_{(i+1) y}\right) v_{i}+\left(\bar{U}_{i}-\bar{U}_{i-1}\right) \eta_{i x}\right\}=0
\end{aligned}
$$

where $u_{n t}$ means $\frac{\partial}{\partial t} u_{n}$. Other similar notations can be interpreted accordingly. 
To search for only normal mode solutions, we can assume

$$
\left.\left(u_{n}, v_{n}, \eta_{n}\right)=\left(\hat{u}_{n}(y), i k \hat{v}_{n}(y), \hat{\eta}_{n}(y)\right) e^{i k(x-c t)}\right)
$$

Substitute Equation (4.2.7) into Equations (4.2.6a-c), drop all " ' signs for convenience, and we have

$$
\begin{gathered}
\bar{U}_{n} u_{n}+\left(\bar{U}_{n y}-f\right) v_{n}+\sum_{i=1}^{n} g_{i} \eta_{i}=c u_{n} \\
-\frac{f}{k^{2}} u_{n}+\bar{U}_{n} v_{n}+\sum_{i=1}^{n}-\frac{g_{i}}{k^{2}} \eta_{i y}=c v_{n} \\
\sum_{i=n}^{N}\left\{T_{i} u_{i}+B_{i} v_{i}+\left(\bar{U}_{i}-\bar{U}_{i-1}\right) \eta_{i}\right\}=c \eta_{n}
\end{gathered}
$$

where

$$
\begin{aligned}
T_{i} & =H_{i}+\bar{\eta}_{i}-\bar{\eta}_{i+1} \\
B_{i} & =T_{i y}+T_{i} \frac{\partial}{\partial y} \\
& =\left(\bar{\eta}_{i y}-\bar{\eta}_{(i+1) y}\right)+\left(H_{i}+\bar{\eta}_{i}-\bar{\eta}_{i+1}\right) \frac{\partial}{\partial y}
\end{aligned}
$$

The lateral boundary conditions are

$$
v_{n}=0, \quad \text { at } y=0, L_{y}
$$

As an example, for the simplest case of two-layer model $(\mathrm{N}=2)$, Equations (4.2.8a-c) become

$$
\left[\begin{array}{cccccc}
\bar{U}_{1} & \bar{U}_{1 y}-f & g_{1} & 0 & 0 & 0 \\
-\frac{f}{k 2} & \bar{U}_{1} & -\frac{g_{1}}{k 2} \frac{\partial}{\partial y} & 0 & 0 & 0 \\
T_{1} & B_{1} & \bar{U}_{1} & T_{2} & B_{2} & \bar{U}_{2}-\bar{U}_{1} \\
0 & 0 & g_{1} & \bar{U}_{2} & \bar{U}_{2 y}-f & g_{2} \\
0 & 0 & -\frac{g_{1}}{k 2} \frac{\partial}{\partial y} & -\frac{f}{k 2} & \bar{U}_{2} & -\frac{g_{2}}{k_{2}} \frac{\partial}{\partial y} \\
0 & 0 & 0 & T_{2} & B_{2} & \bar{U}_{2}
\end{array}\right]\left[\begin{array}{c}
u_{1} \\
v_{1} \\
\eta_{1} \\
u_{2} \\
v_{2} \\
\eta_{2}
\end{array}\right]=c\left[\begin{array}{c}
u_{1} \\
v_{1} \\
\eta_{1} \\
u_{2} \\
v_{2} \\
\eta_{2}
\end{array}\right]
$$


We will use a finite difference method in $y$ with a staggered scheme to solve this linear eigenvalue problem. 


\subsection{Comparison of the SW and QG Six-Layer Models}

In the SW six-layer model, the depths of the interfaces, velocity profiles and topography are the same as in QG case (see Figure 3.5) and Figure 3.7).

Figure 4.1 shows the results for temporal instabilities in the QG and SW six-layer models. The dashed curves represent the results of the QG six-layer model we have studied in the Chapter 3, and solid curves the SW six-layer model. The main modes from the two models are similar in wavelengths $(349 \mathrm{~km}$ in QG6 vs $363 \mathrm{~km}$ in SW6) except the maximum growth rate (0.063/day) in QG model is about $40 \%$ higher than that in SW case (0.044/day). This agrees well with previous study by Orlansky (1969) who shows in a two-layer model that the growth rates and corresponding wave numbers of unstable modes decrease when the Rossby number increases. As we can see from the upper panel of Figure 4.1, the difference between the phase speeds of the main modes in the QG and SW model is relatively small compared to the difference in growth rates. As far as the main mode is concerned, there appears to be a quantitative difference between the QG and SW models in growth rate but no qualitative difference in the shape of the curve of the growth rate and the phase speed of the mode.

In both models, there are two more significant modes, which have higher phase speeds and wavenumbers than the main modes. Numbered in the order of wavenumber at peak, the second mode in SW model seems to correspond to both the second and third modes in QG model as seen in the phase speeds. The third mode in SW model is not present in the QG model.

Figure 4.2 is the pulse instability version of the comparison. As we can see in Figure 4.2, the characteristics of the main modes in the SW and QG models are comparable except that the main mode in SW model truncates at lower group velocity 

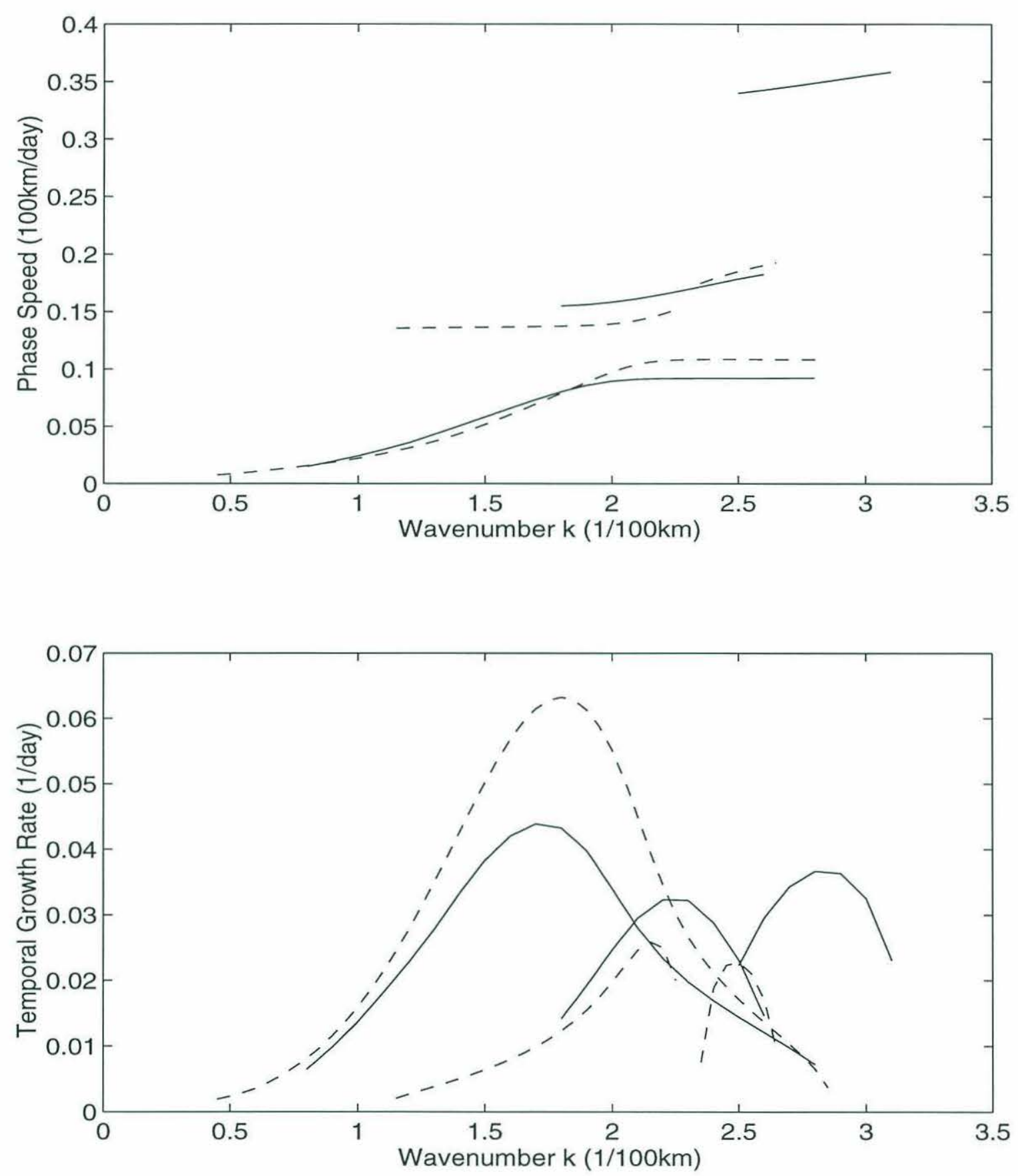

Figure 4.1: The results of temporal instabilities corresponding to QG and SW six-layer models. The solid curves represents the results of the SW model, and dashed curves the QG model. 

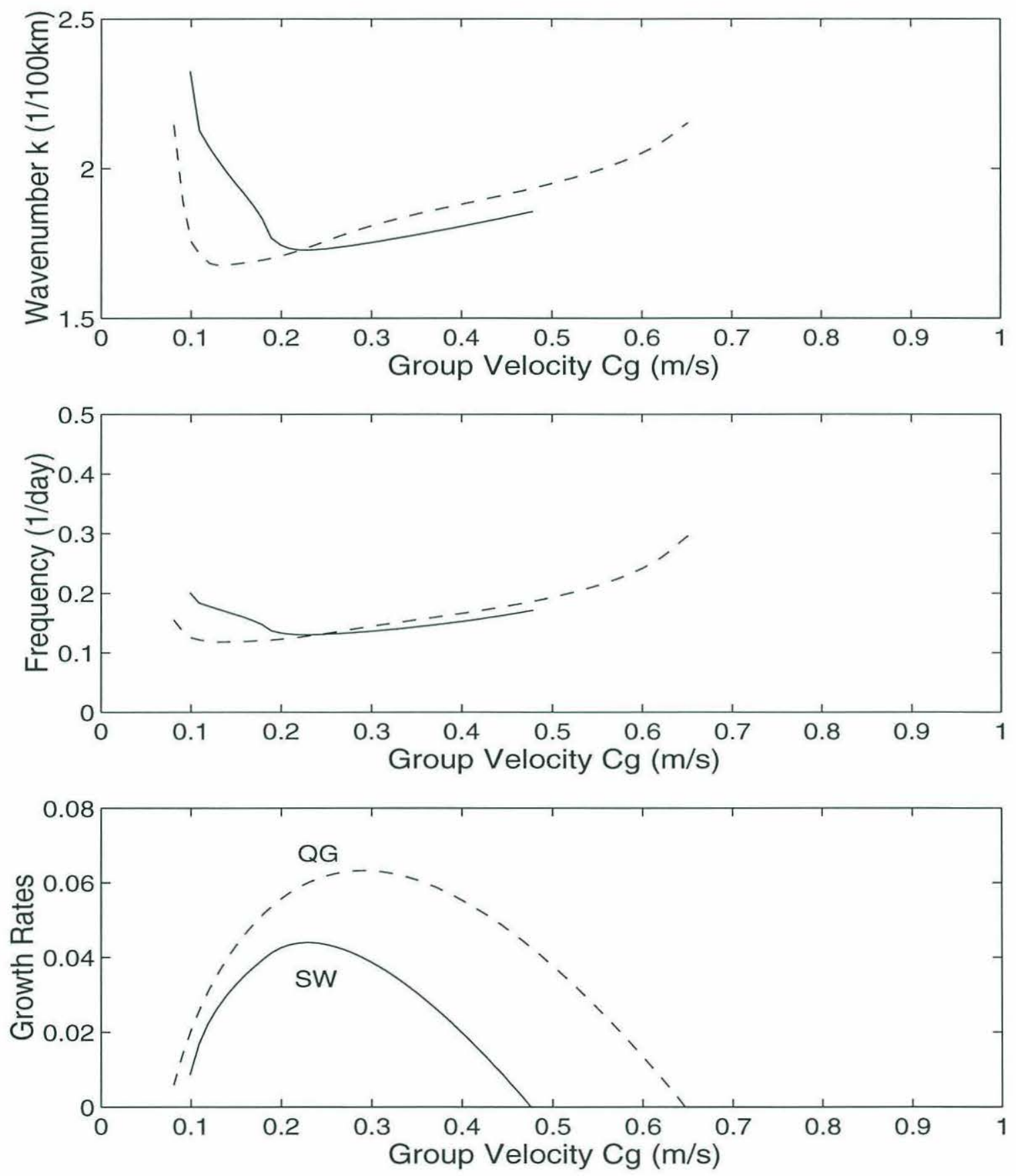

Figure 4.2: The main modes of pulse instabilities corresponding to QG and SW six-layer models. The solid curves represents the results of the SW model, and dashed curves the QG model. 
than the main mode in the QG model, so that the former only corresponds to the first part of the latter. This can be seen more clearly in Figure 4.3, which also plots the growth rates of individual peaks. Despite the difference in the maxima of the envelope growth of the main modes, the growth rates of individual peaks agree very well between the two models. This appears to be coincidental; the curve of individual peak growth in one model happens to pass very nearly through the maximum of the envelope growth in the other model, so the two curves agree at both of these points. This implies that when we compare the growth rates of individual peaks from the models with observations, the difference between the QG and SW models does not tend to show up as clearly as when we compare those of envelopes with observations.

Figure 4.4 shows the three most unstable modes of pulse instabilities in QG and SW models. In the SW case, the second mode dominates the first mode when group velocity is greater than $0.35 \mathrm{~m} / \mathrm{s}$; whereas, in the QG case, the first mode is strongly dominant over most of the range where the growth rates are high. Thus the comparison between QG and SW is more complex than Figure 4.2 might suggest. To determine which mode the fastest growing peak actually comes from, we need to compare the maximum growth rates of individual peaks in the regions where their corresponding envelope growth rates dominate. Figure 4.5 shows the growth rates of individual peaks corresponding to the three most unstable modes. The maximum growth rate of an individual peak from the first mode turns out to be $0.105 /$ day at group velocity of $0.35 \mathrm{~m} / \mathrm{s}$ and period of 40 days, while that from the second mode is $0.091 /$ day at group velocity of $0.50 \mathrm{~m} / \mathrm{s}$ and period of 16 days. Moreover, the maximum growth of an individual peak from the third mode is $0.110 /$ day at $C_{g}=.71 \mathrm{~m} / \mathrm{s}$ and corresponds to a period of 6 days. The maximum growth rates of individual peaks from the three modes are actually comparable and it is difficult to differentiate between them. However, because of the difference in group velocities, they will appear at different locations and have different wavelengths, and may therefore all be observable. 

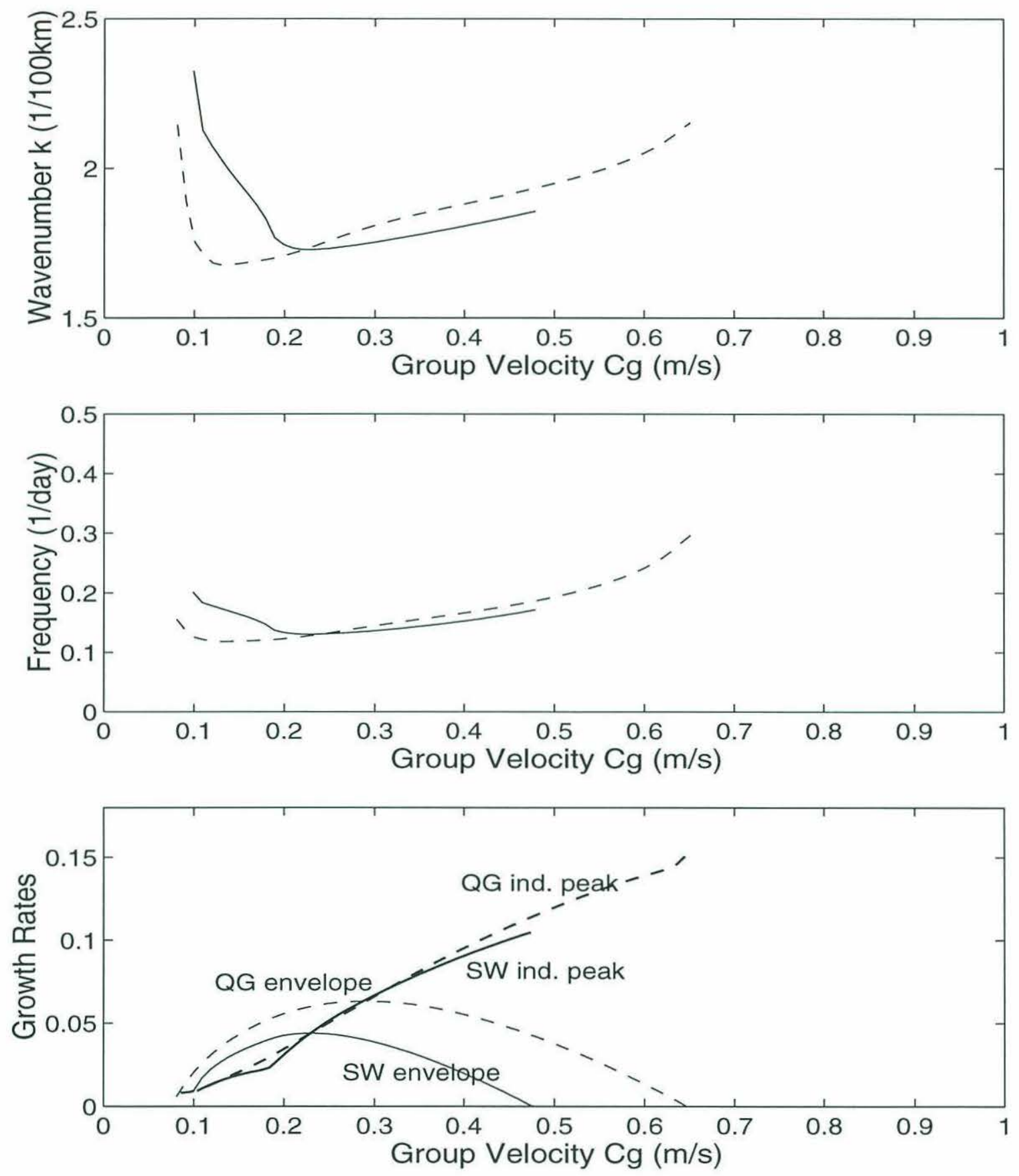

Figure 4.3: Growth rates, frequencies and wavenumbers corresponding to the most unstable modes in QG and SW six-layer models. 

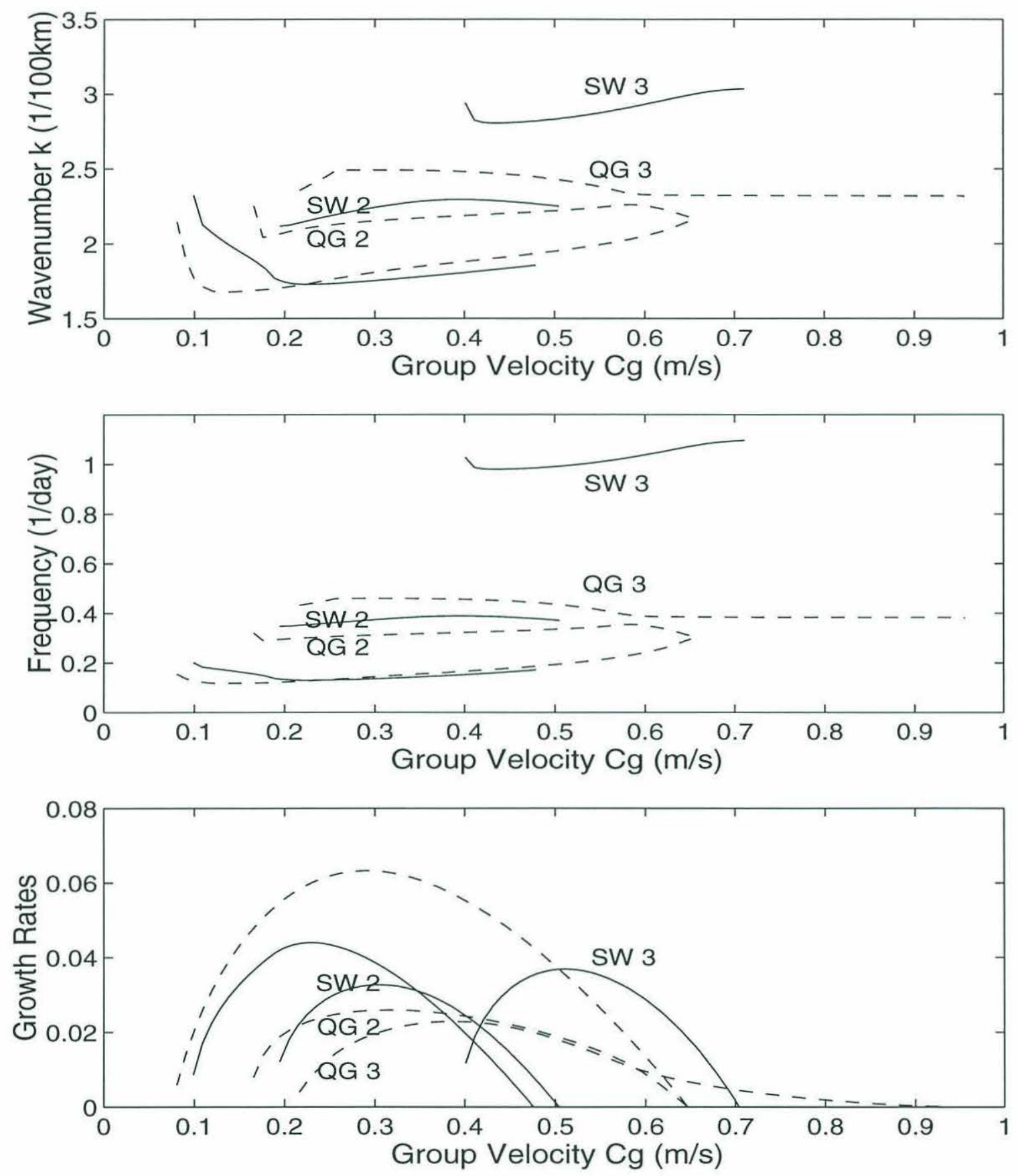

Figure 4.4: Comparison of the three most unstable modes of pulse instabilities in QG and SW six-layer models. The solid curves represents the results of the SW model, and dashed curves the QG model. 

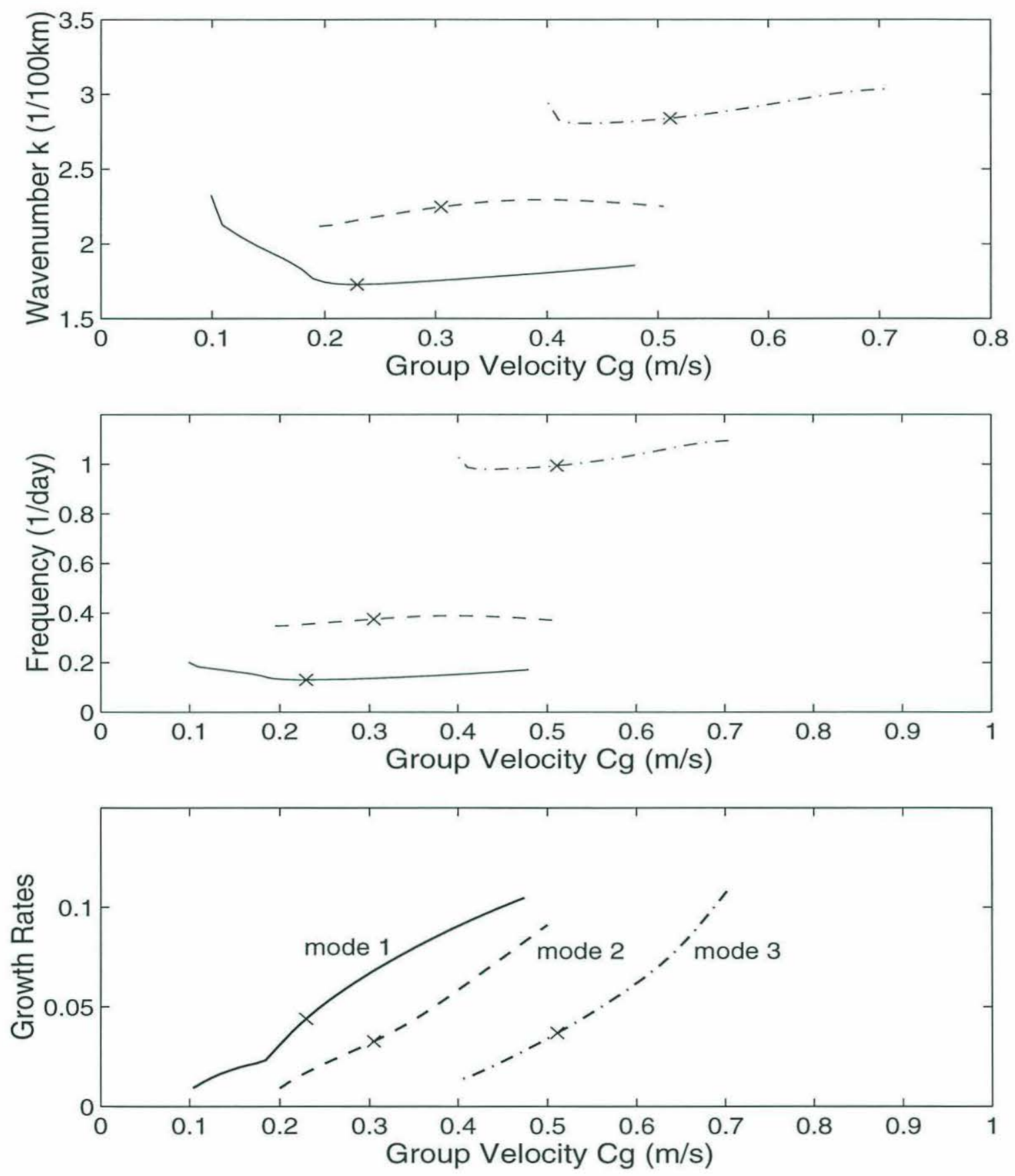

Figure 4.5: Growth rates, frequencies and wavenumbers corresponding to the three most unstable modes in the SW six-layer model. 
Xue (1991a) also carried out similar instability analysis of the Gulf Stream on the South Atlantic Bight in a primitive model. She only studied temporal instability. The most unstable waves in her models with topography have wavelengths between 215 and $225 \mathrm{~km}$ and e-folding times of about 3 days. This is very different from the most temporally unstable wave in this model, which has a wavelength of $350 \mathrm{~km}$ and an e-folding time of 23 days. This large difference is probable due to the different basic states and topography used in her model (intended for the Stream before Cape Hatteras) and mine.

\subsection{Comparisons With Observations and Conclu- sion}

In this section, I will draw connection between our model results and observations, and conclude our findings in the chapter. In the comparison with observation, I will focus on the studies by Lee and Cornillon (1994a,b) since only their work distinguishes the fastest growing wave from most energetic wave in a well-defined way, to the best of my knowledge.

Lee and Cornillon used two-day composite sea surface temperature (SST) images obtained from the thermal infrared (IR) channels of the Advanced Very High Resolution Radiometer (AVHRR) flown on the NOAA polar-orbiting satellites. From these satellite images, they digitized the path of the Gulf Stream from Cape Hatteras (about $75^{\circ} \mathrm{W}$ ) to $45^{\circ} \mathrm{W}$. The path of the Gulf Stream is defined by the sharp SST gradient along the northern edge of the stream. After removing the mean position (a smooth curve) of a path, the positions $y(x, t)$ of the Gulf Stream as a function of $(x, t)$ were obtained. They then performed spectral analysis to find the most energetic wave band. They used FFT in the time-frequency transform. In the position-wavenumber transform, they used either FFT or autoregression method. Using the former resulted 
in the most energetic wave of $427 \pm 70 \mathrm{~km}$ in wavelength and 46 days in period. Using the latter gave the same results except that the error estimate in wavelength became $\pm 16 \mathrm{~km}$.

The fastest growing wave was obtained by a peak tracking technique. Given a path of the Gulf Stream, they repeated three-point averaging a few times to get a heavily-averaged path. This path intersects the original path, and these intersections define the nodes between crests and troughs. For an individual peak, the wavelength associated is defined as twice of the distance between the two adjacent nodes. If the positions of a peak are $x_{1}$ and $x_{2}$, and amplitudes $A_{1}$ and $A_{2}$, at the time $t_{1}$ and $t_{2}$ respectively, the growth rate and phase speed between $t_{1}$ and $t_{2}$ are defined as $\frac{1}{A_{1}} \frac{A_{2}-A_{1}}{t_{2}-t_{1}}$ and $C_{p h}=\frac{x_{2}-x_{1}}{t_{2}-t_{1}}$, respectively. These values are assigned to the average position $\frac{x_{2}+x_{1}}{2}$ and the average time $\frac{t_{2}+t_{1}}{2}$. To be consistent, the wavelengths and amplitudes of the same peak at $t_{1}$ and $t_{2}$ are also averaged to get the mean assigned to the time $\frac{t_{2}+t_{1}}{2}$. They used bins of wavelengths $(50 \mathrm{~km}$ interval) to group the observational data and average the growth rates in each bin. Their analysis in this way has found the fastest growing wave of $350 \mathrm{~km}$ in wavelength and 40 days in period.

In Chapter 2, I have emphasized that even in the case of pulse instability, the amplitude and energy of each Fourier component still grows as temporal instability theory predicts. This suggests that the most unstable wave from temporal instability or maximum of envelope growth from pulse instability should be compatible with the most energetic wave Lee and Cornillon described, if the nonlinearity in the observation is not considered. And the way they calculated the fastest growing wave is straightforward and also compatible with the fastest growing individual peak wave in my models.

The following table summarizes the differences between the most unstable wave and the fastest growing wave described by Lee and Cornillon (1994b) as well as those predicted from our models. Although Lee and Cornillon attributed the 
differences to nonlinearity, I will first compare their results with linear models and return to the nonlinearity issue in Section 6.3.

\begin{tabular}{|l|c|c|c|c|c|c|}
\hline & \multicolumn{3}{|c|}{ most energetic wave } & \multicolumn{3}{c|}{ fastest growing wave } \\
\cline { 2 - 7 } & $\mathrm{L}(\mathrm{km})$ & $\omega_{i}(1 /$ day $)$ & $\mathrm{T}$ (days) & $\mathrm{L}$ & $\nu_{i}(1 /$ day $)$ & $\mathrm{T}$ \\
\hline Lee and Cornillon & $427 \pm 70$ & .032 & 46 & 350 & .063 & 40 \\
\hline SW6 & 363 & .044 & 48 & 339 & .105 & 37 \\
\hline QG6 & 349 & .063 & 44 & 293 & .151 & 22 \\
\hline
\end{tabular}

When it comes to the difference between the most unstable and the fastest growing modes, the results of different models spread widely and there is tradeoff between how well a model can explain the period and the wavelength, taking observations as given (i.e. without error). For example, in the QG six-layer model, the ratio of the wavelength of the fastest growing wave to that of the most unstable wave is $84 \%$, which compares well with the ratio observed $(82 \%)$. However, the ratio of the period of the fastest growing wave to that of the most unstable wave is only $50 \%$, too small compared the ratio observed (87\%). In the six-layer SW model, on the other hand, the ratio in wavelengths is $93 \%$, larger than the observed $(82 \%)$; the ratio in periods is $77 \%$, which agrees better than the QG model with that observed (87\%). The ratio of the growth rate of the fastest growing wave to that of the most unstable wave is $2.0,2.4$, and 2.4 from the observation, the QG and SW six-layer models, respectively. The ratios of the growth rates seem to be in reasonable agreement.

Despite the discrepancy among the models and the observation, it appears to be a robust common feature that the fastest growing wave has higher growth rate, wavenumber and frequency than the most unstable wave. And the differences between the most unstable and the fastest growing waves in the models seem able to explain substantial parts of the differences observed.

The QG six-layer model overestimates substantially the maximum growth rate compared to the SW six-layer model (e.g. $40 \%$ higher in QG6 than that in SW6). This is consistent with the previous study by Orlansky (1969) that finite Rossby 
number reduces the growth rates of unstable waves. However, QG and SW models give comparable wavelengths and phase speeds of the main unstable modes (e.g. $349 \mathrm{~km}$ and $7.9 \mathrm{~km} /$ day in QG6 vs $363 \mathrm{~km}$ and $7.5 \mathrm{~km} /$ day in $\mathrm{SW} 6)$. The growth rates of individual peaks in the two models agree very well as functions of the group velocity, though the maximum growth rates are still significantly different. The conclusion from this comparison is that the QG model gives results quantitatively different from those from the SW model, but there is no qualitative difference between the two models in term of the first most unstable modes. 


\section{Chapter 5 \\ The Instabilities of the Gulf Stream-Numerical Simulation}

\subsection{Introduction}

In Chapter 4, I have compared the pulse instability from the QG and SW six-layer models with the observational analysis by Lee and Cornillon (1996b). The conclusion is that the differences between the most energetic and the fastest growing waves in the models appear able to explain substantial parts of the observed differences. Before we conclude that pulse instability is the most appropriate model for Gulf Stream movements, we need to address a couple of issues. The first is whether there is enough time for pulse instability to establish itself in the Gulf Stream because we are using an asymptotic solution which requires that the time after the initial perturbation be large. Since the center of a wave packet moves at a specific group velocity, the issue can be translated into whether there is enough space for the pulse instability to occur. This issue will be addressed in Section 5.5, where I consider the development of a single pulse in a two-layer model with jet structure and a sloping bottom.

The second issue concerns an assumption implicit in the comparisons between pulse instability from the models and the observed motions. The assumption is that the initial disturbance is a pulse and no further perturbation is added in the region. This seems unlikely to be the case in the Gulf Stream. To address the effect of continuous disturbances in the Gulf Stream as well as the first issue, I will use a forced two-layer numerical model in this chapter. For comparison, I will first study a two-layer analytical model which uses the exact same parameters as the numerical model. 
The reason I study a two-layer numerical model instead of six layers is the computational difficulty. As we already know from the spread ratio analysis in Section 3.3 neither form of the two-layer model, with flat bottom or real bottom, will take the topographic effect into account properly. However, since we already know the dispersion relation of the six-layer model, we may choose the slope of the bottom and velocity structure in a two-layer model so that its dispersion relation best resembles that of the six-layer model. It is important to keep in mind that without the results from the QG six-layer model, we could not choose the parameters in the two-layer model from the section data meaningfully to get a reasonable dispersion relation.

The numerical model has three dimensions instead of two dimensions in the analytical model, the third dimension being the along-stream direction. Because mean flow and waves are moving along that direction, it needs to be much longer than the cross-stream width of the channel model. As discussed in the next section, the parameters of the two-layer model and especially the topography will be adjusted so that the instability properties are similar to the QG six-layer model.

I will use the satellite data analyzed by Lee and Cornillon (1996a) to force the numerical model in the upper stream, and investigate how differently disturbances evolve compared to the one single pulse idealized case studied in this chapter.

\subsection{A Two-Layer QG Analytical Model}

Because of computational difficulty in multilayer numerical models, I will focus on a two-layer numerical model in this chapter. To prepare for the comparison between numerical runs and analytical results, a two-layer analytical model is first studied here. For the model to have a dispersion relation as close to the Gulf Steam situation as possible, I search for a two-layer model with proper topography and 


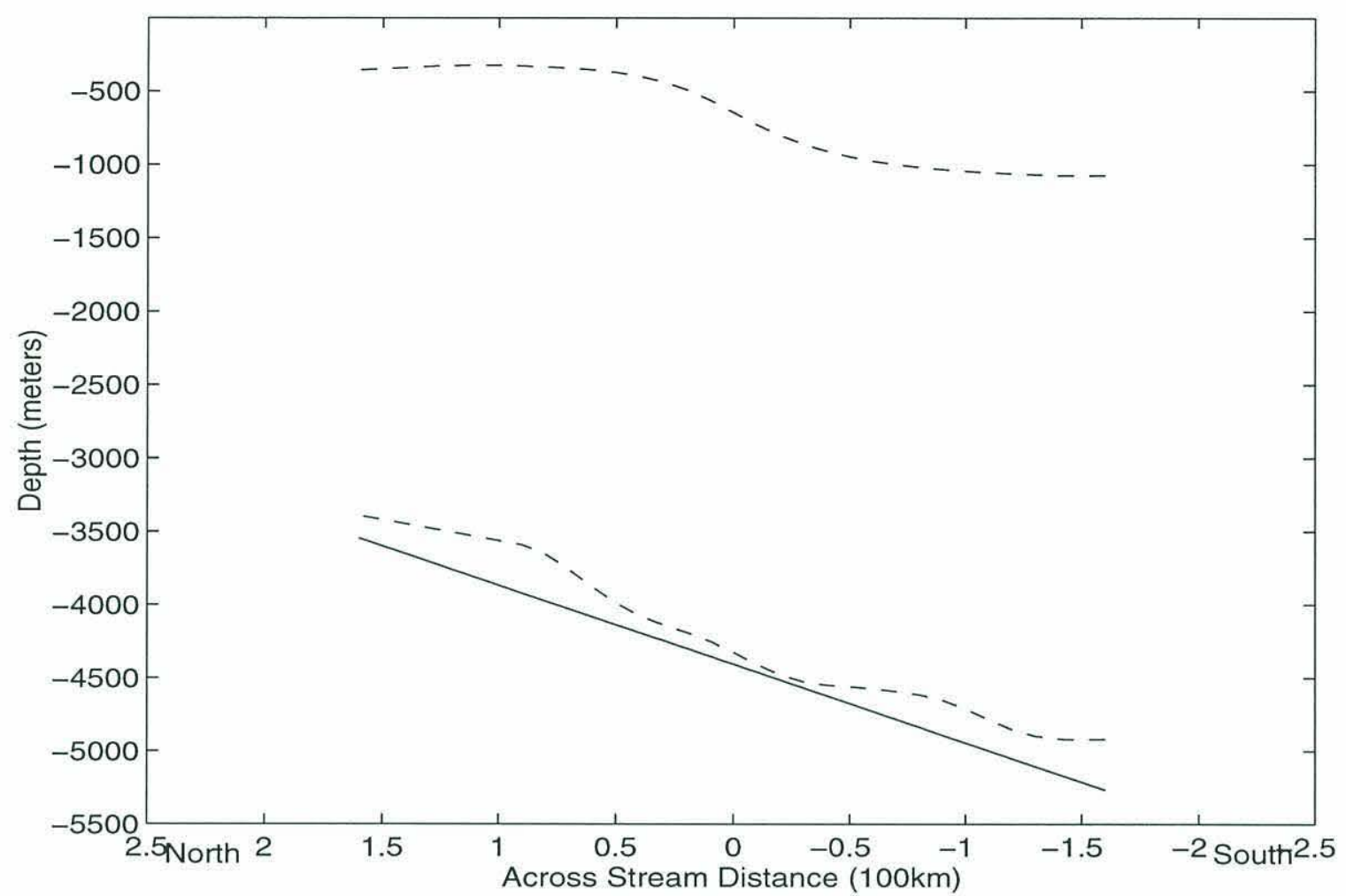

Figure 5.1: A two-layer model with a sloping bottom (solid curve). Also plotted is the topography used in the multiple layer model (lower dashed). Upper dashed curve is the interface between the upper and lower layers.

velocity structure which best approximates the dispersion relation of the six-layer model.

As we have found in Chapter 3, a two-layer model with a realistic topography will overestimate the effect of topography. In this two-layer model, I take the alternative to use a sloping bottom and choose the slope so the maximum growth rate of the main mode in the model will match the maximum growth rate of the main mode in the QG six-layer model. Figure 5.1 shows the configuration of a two-layer model we will use. The solid curve is a sloping bottom. Its slope is determined to be 0.0054 . Also plotted is the topography used in the multiple-layer model (lower dashed). The upper dashed curved is the interface between the upper and lower layers. 
In two-layer models, we do not fit the velocity structure with a Gaussian profile since the purpose is to match the dispersion relations in the six-layer model and it turns out the Gaussian fit does not make the best match. Figure 5.2 shows the layeraveraged velocity profile calculated from CTD data (Hall and Fofonoff, 1993) for the two-layer model. The region marked with '?' sign in the figure has an unusual small scale structure, which arises from a single data point and does not appear in other cross-stream profiles. This may be due to an eddy or the lack of barotropic velocity component. In order to see the effect of the small scale structure on instabilities, I have modified the velocity profile slightly, as shown in Figure 5.3.

Figure 5.4 shows the instabilities calculated for these two profiles (using sloping bottom), with dash-dotted curves associated with the original profile and solid curves with the modified one. We can see that the peak of growth rate at $k=2.9$ (or a wavelength of $217 \mathrm{~km}$ ) has been significantly reduced by smoothing the profile and becomes negligible. From the phase speed associated with this peak, we can identify that this peak as arising from barotropic instability in the top layer. By smoothing the velocity profile slightly on the edges of the jet, the peak has been reduced more than $70 \%$. Because this peak is so sensitive to the profile and the structure in the original profile in this region arises from a single data point, we do not believe that the peak at $\mathrm{k}=2.9$ is a robust feature in the Gulf Stream. Therefore, we will use the modified profile hereafter.

Figure 5.5 shows temporal instability in the model. The upper panel shows phase speeds vs real wavenumber $k$, while the lower panel gives temporal growth rate $\omega_{i}$. For comparison, the results from the QG six-layer model are represented with dashed curves. The two-layer model agrees well with the six-layer model in the main mode. The maximum growth rates in the two models are the same because the slope in the two-layer model is such chosen to best match the maximum growth in the six-layer model. Moreover, the shapes of the main modes in the two models 


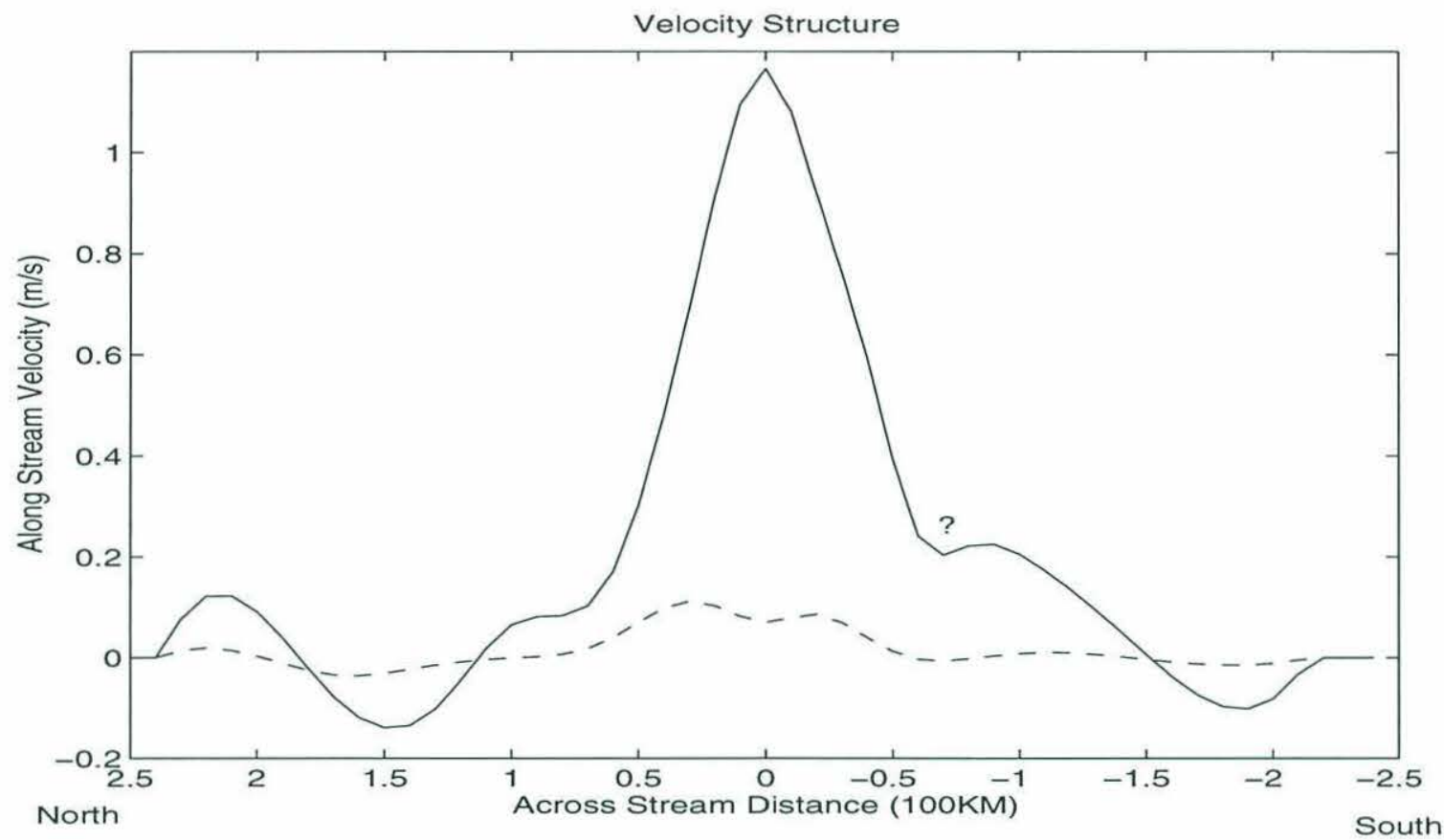

Figure 5.2: Layer-averaged velocity profile. Solid line: top layer velocity, dashed line: bottom layer velocity.

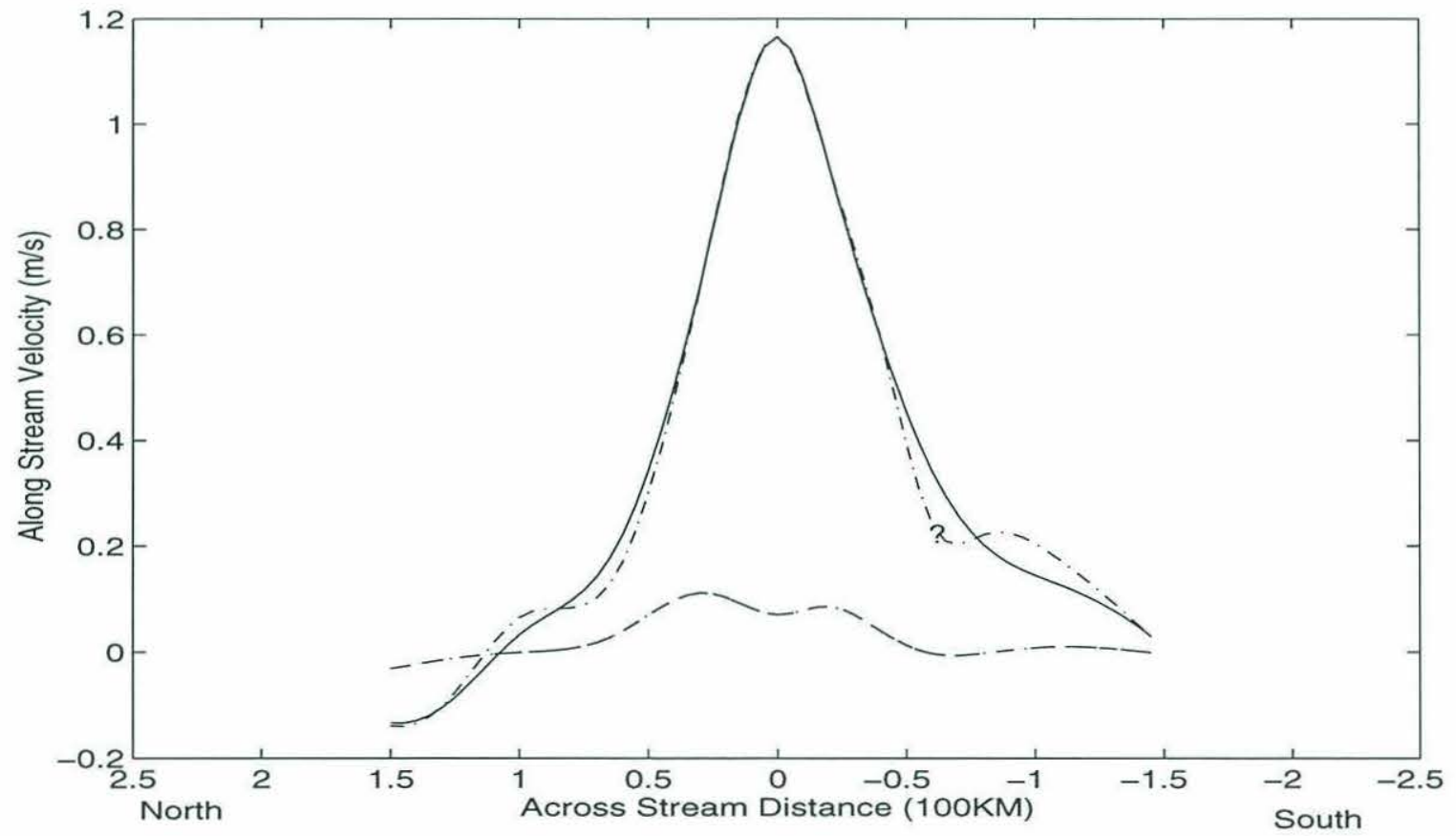

Figure 5.3: Dash-dotted lines represent the original velocity profile and solid lines the modified velocity profile. 

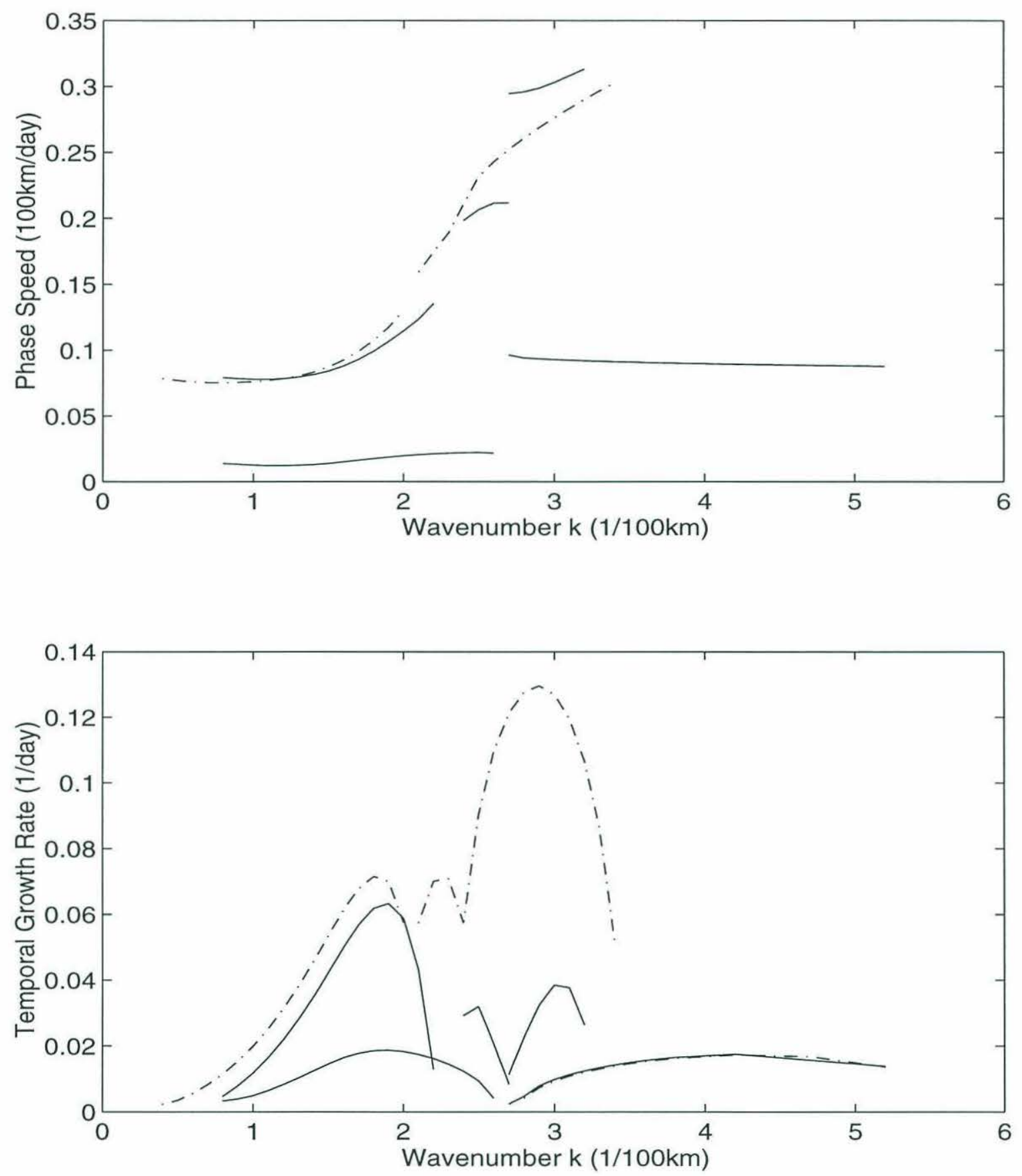

Figure 5.4: Upper panel: Phase speeds vs real wavenumber $k$. Lower panel: Temporal growth rate $\omega_{i}$ vs real wavenumber $\mathrm{k}$. Dash-dotted curves for the original profile and solid ones for the modified profile. 
are similar. This is also true with wavenumbers and frequencies in pulse instability calculation shown in Figure 5.6. Although for given group velocity, the two-layer model has higher wavenumber and frequency, the slopes of the curves compare well with the six-layer model. The figure only compares the most unstable modes in each models. The lower panel shows how the growth rates of individual peaks differ from corresponding envelope growth rates. It is clear that the patterns are similar in the two models.

\subsection{Temporal, Spatial and Pulse Instabilities in a Two-Layer Model}

The purpose of this section is to prepare for comparisons between analytical and numerical models in later sections. Since we have already chosen parameters in this analytical model so that the dispersion relation for the primary mode resembles that of the QG six-layer model, the differences between temporal, spatial and pulse instabilities in this model are similar to those in QG6 and will not repeat the similar comparisons here. However, we do need to examine spatial instability and to consider other modes as well.

The results of temporal and pulse instabilities have been shown in last section (Figure 5.5 and Figure 5.6), but we redraw the corresponding figures here for clarity. Figure 5.7 shows three most temporally unstable modes. The peak with largest growth rate has a wavenumber of about 1.9 (with unit: $1 / 100 \mathrm{~km}$ ), or a wavelength of $334 \mathrm{~km}$. Its maximum growth rate .063 corresponds to an e-folding time of 16 days. The phase speed and group velocity right at the peak are $12 \mathrm{~cm} / \mathrm{s}$ and $29 \mathrm{~cm} / \mathrm{s}$. Its period is 32 days. The second and third largest peaks with $k=3.1$ and 2.5 are fast moving waves concentrated at the upper layer of the Stream. 

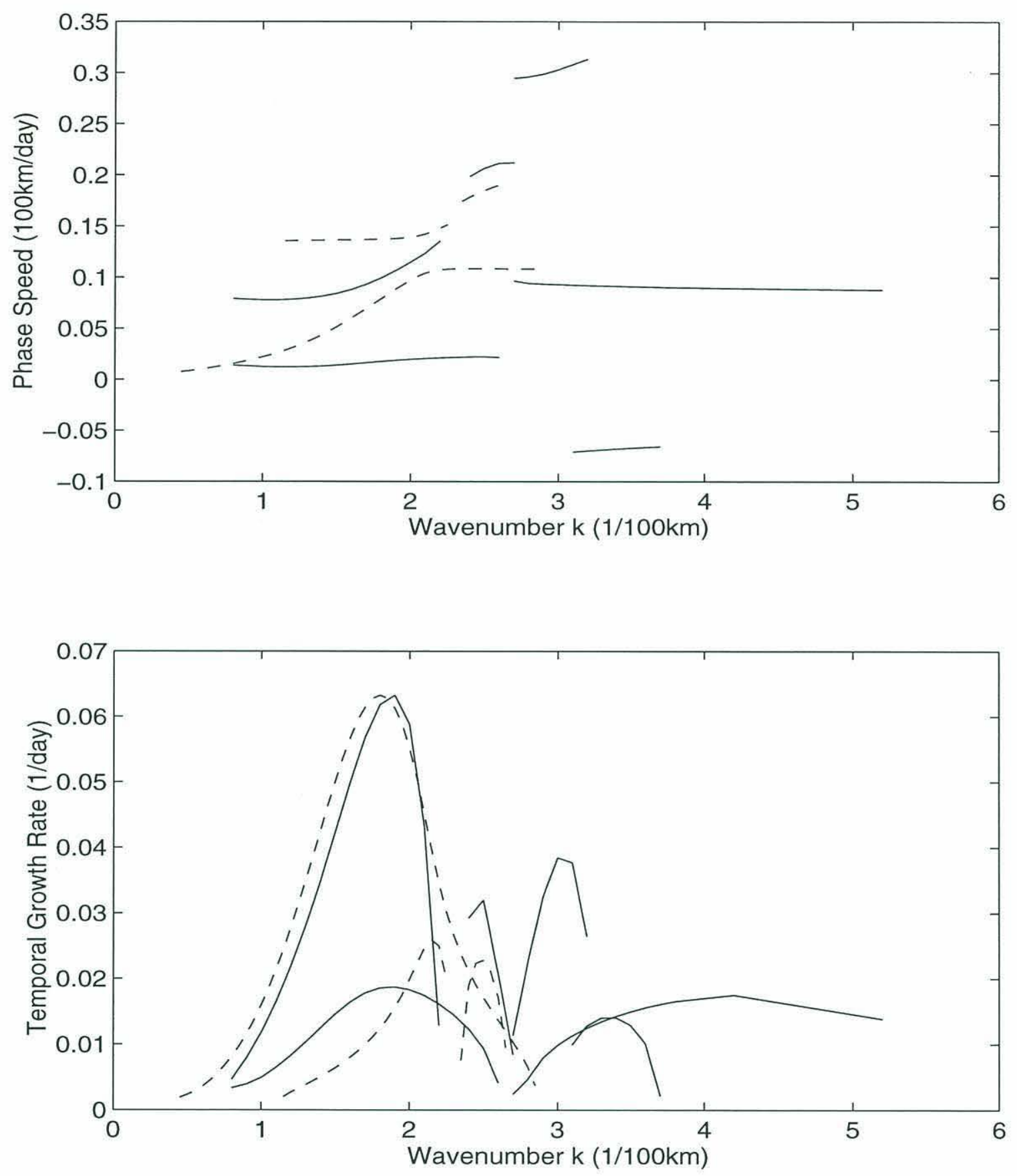

Figure 5.5: Temporal instability in the two-layer model with a sloping bottom. Upper panel: Phase speeds vs real wavenumber $k$. Lower panel: Temporal growth rate $\omega_{i}$ vs real wavenumber k. Solid curves correspond to the two-layer model. For comparison, the results from QG six-layer model are represented with dashed curves. 

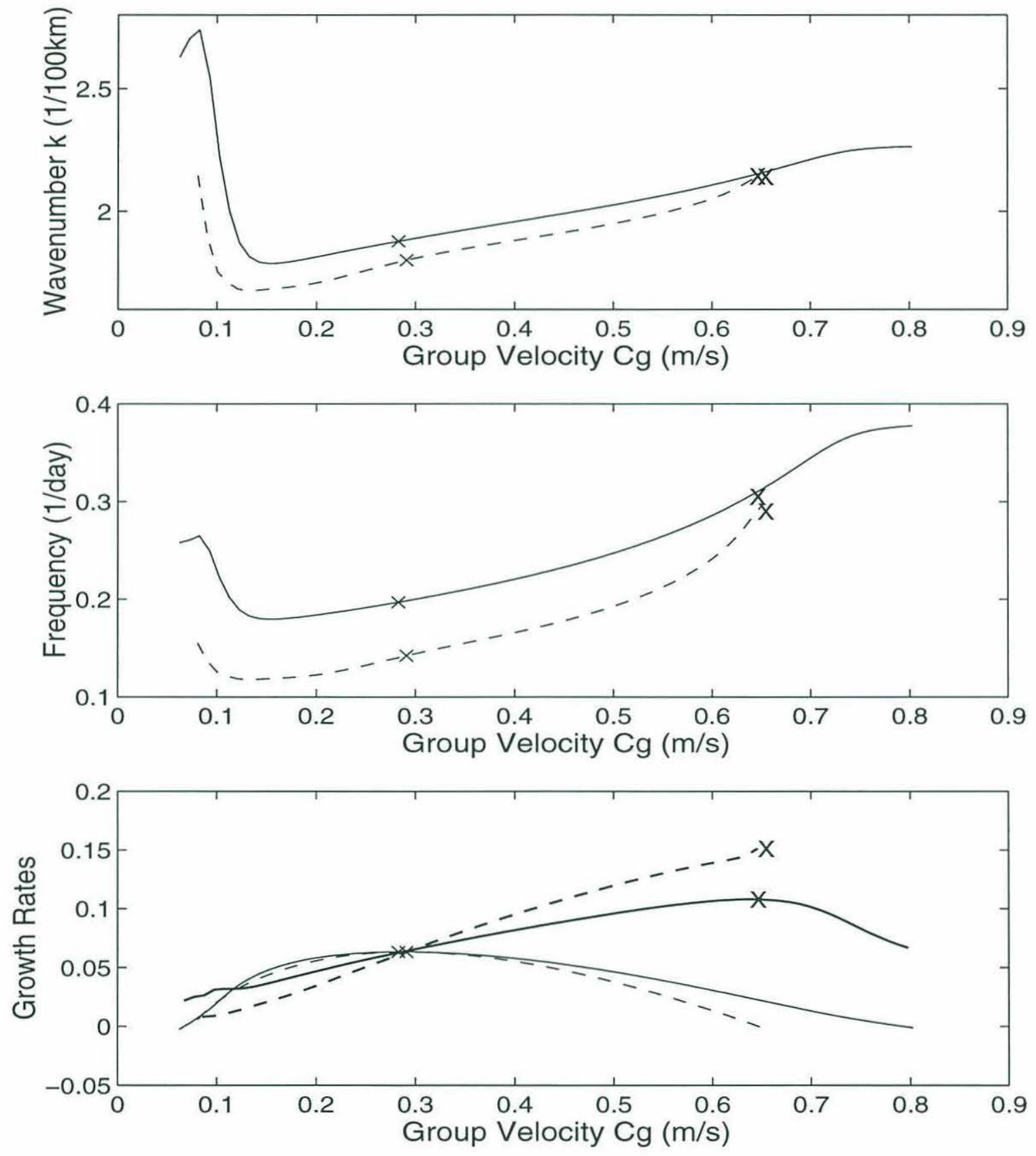

Figure 5.6: The results of pulse instabilities corresponding to the two and six-layer models. Solid curves correspond to the two-layer model. For comparison, the results from QG six-layer model are represented with dashed curves. 

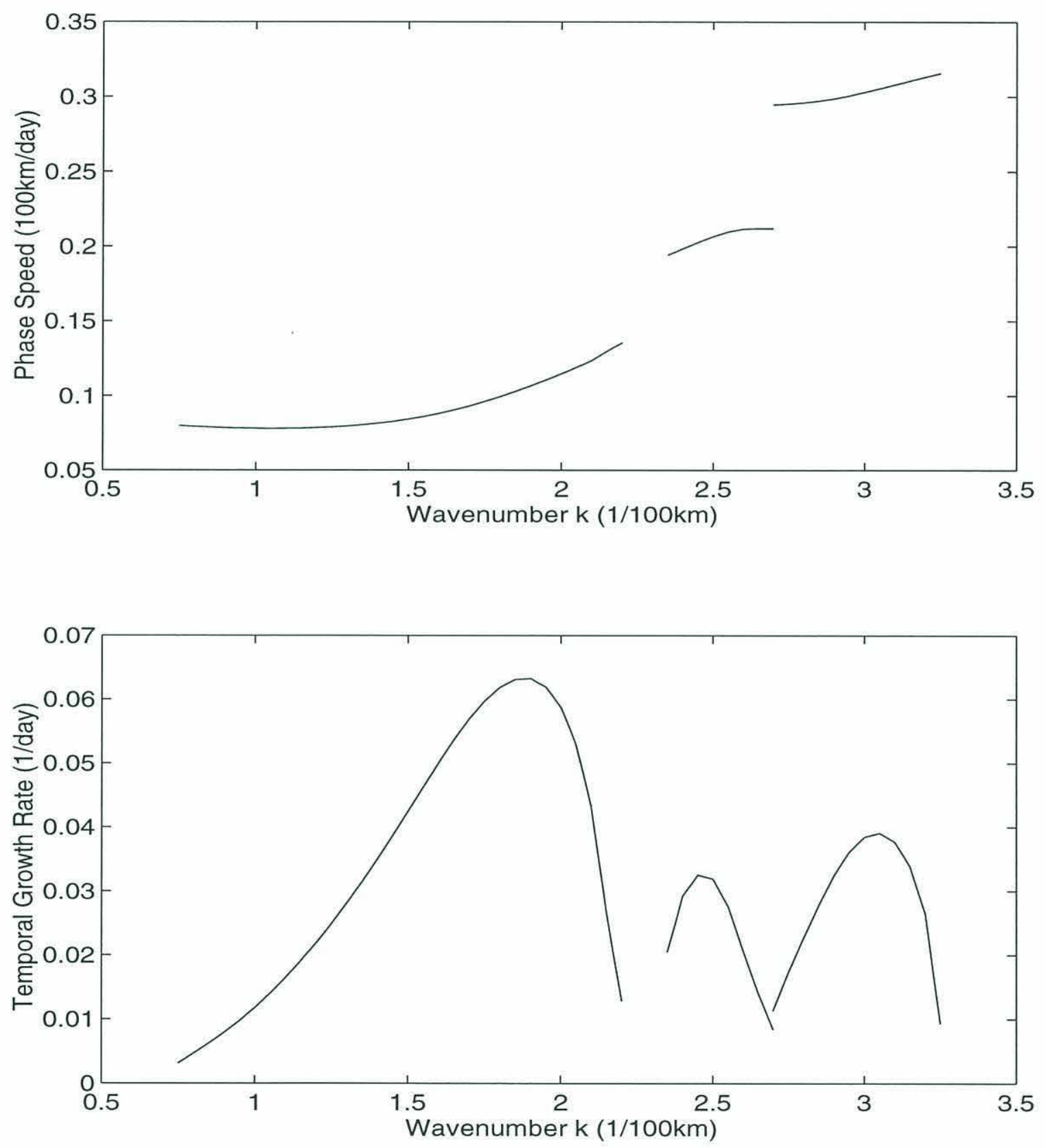

Figure 5.7: Temporal instability in the two-layer model with a sloping bottom. Upper panel: Phase speeds vs real wavenumber $k$. Lower panel: Temporal growth rate $\omega_{i}$ vs real wavenumber $k$. 
The upper panel of Figure 5.8 shows the spatial growth rates of the first three modes in spatial instability as a function of forcing frequency. The maximum growth rate of the first mode is about four times of those of the second and third modes. The second and third modes are numbered in term of wavenumbers and frequencies. The three modes do not overlap in the frequency domain. It is important to remember that it is the frequency of forcing that determines which mode will dominate in a system, not the magnitude of the maximum growth rates of modes. In spatial instability, given a forcing frequency, there will be a dominant wave which moves at constant phase speed determined by dispersion relation. The spatial growth rate multiplied by the phase speed will give the growth rates of individual peaks, which are shown in the lower panel of Figure 5.8. The three modes which do not overlap in frequency domain now overlap in part in wavenumber domain. It is interesting to note that the third mode has a maximum growth rate about $75 \%$ of that of the first mode when one follows an individual peak, whereas the corresponding spatial growth rate is only about $25 \%$ of that of the first mode.

Figure 5.9 and Figure 5.10 show the results from pulse instability for the three most unstable waves. In all the panels, the horizontal coordinate is group velocity, which is multiplied by the time to derive the position of the individual peak from where the disturbance originated. The upper and lower panel of Figure 5.9 show the growth rates of individual peaks and the envelopes. ' $x$ ' and dark ' $x$ ' marks indicate the properties corresponding to the maximum growth rates of envelopes and individual peaks, respectively. From the previous chapters, we already know that the growth rate of an individual peak closely depends on its position relative to the peak of the envelope and can be very different from that of the envelope. In this model, an individual peak in the leading edge of the wave packet can grow as fast as $170 \%$ the rate of the envelope growth (for the most unstable mode). For the second or third mode, that ratio is about fourfold. A peak in the leading edge can grow several times as fast as another peak in the trailing edge. 

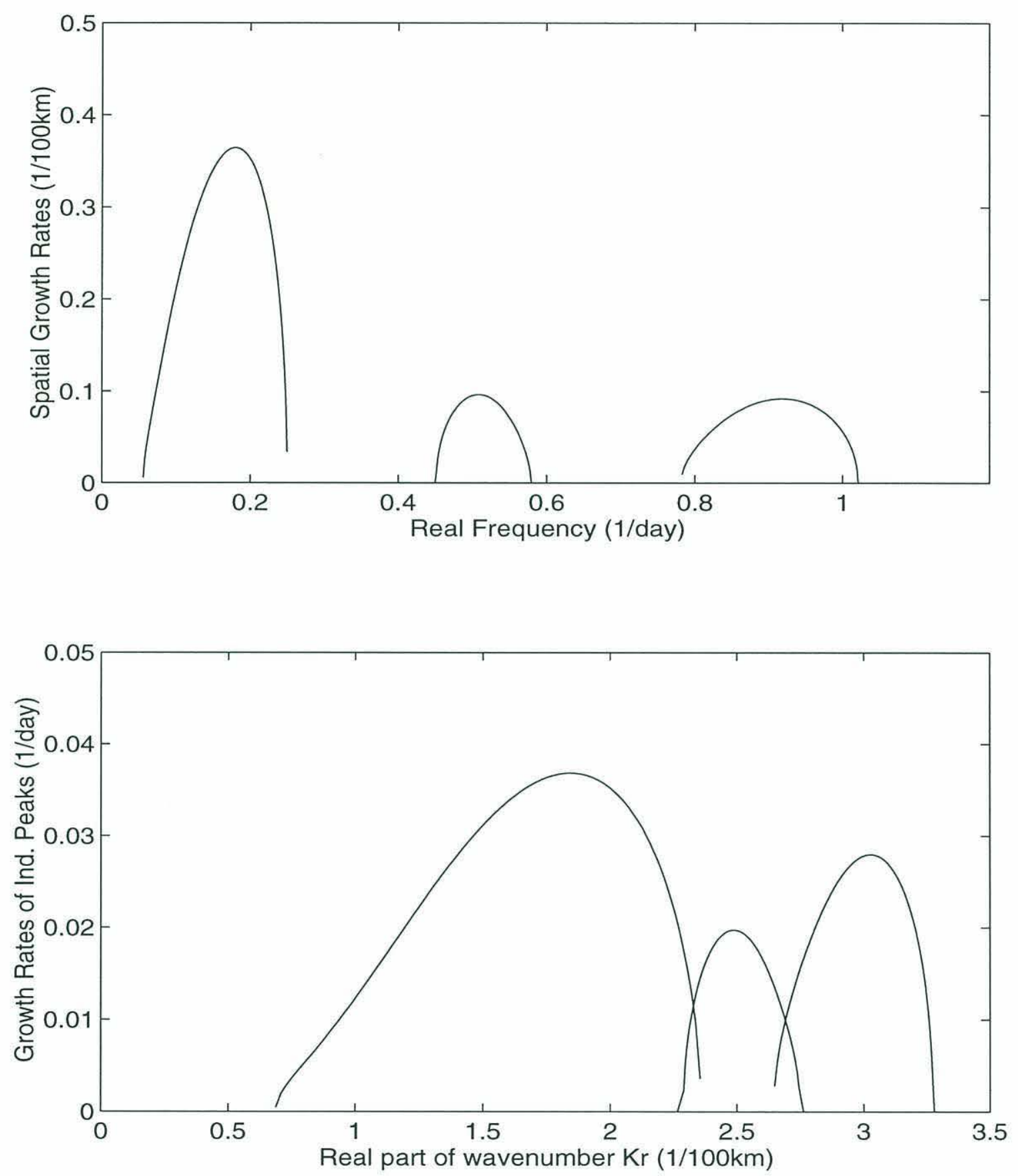

Figure 5.8: Spatial instability: Upper panel: spatial growth rates. Lower panel: growth rates of individual peaks. 

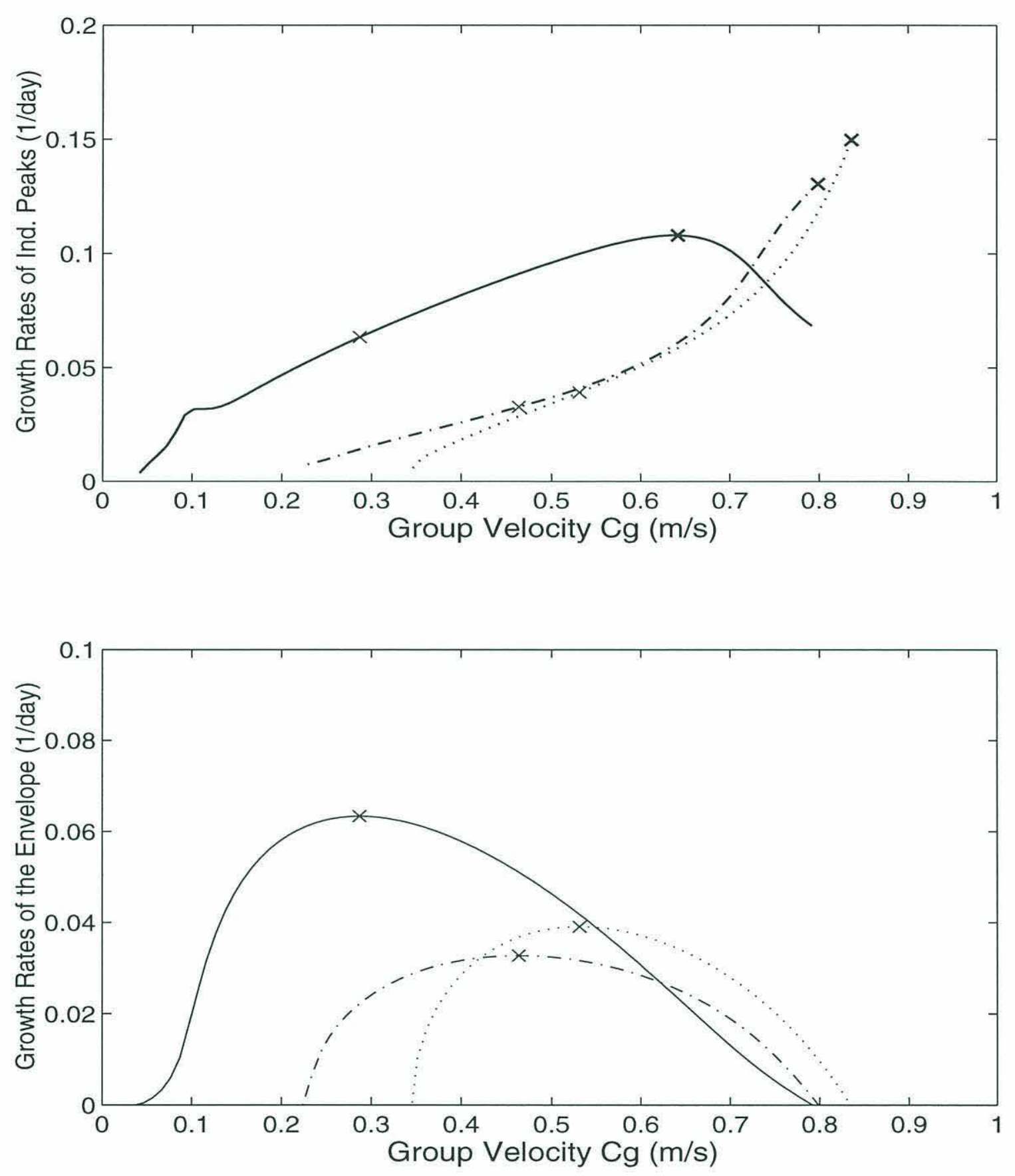

Figure 5.9: Three most unstable modes in pulse instability: Upper panel: growth rates of individual peaks. Lower panel: growth rates of the envelopes. 

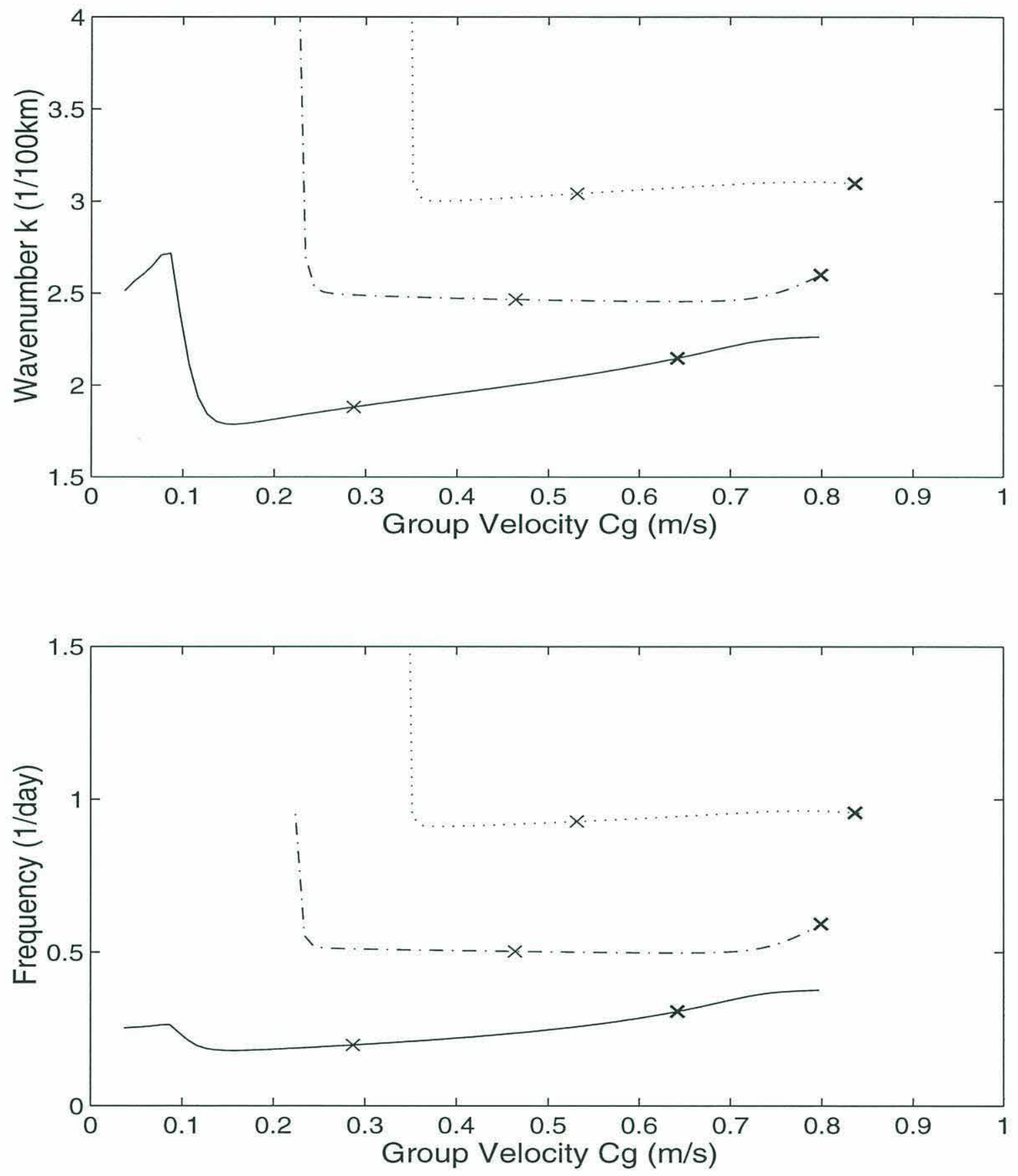

Figure 5.10: Pulse instability: wavenumbers and frequencies. 
The upper and lower panels in Figure 5.10 show the corresponding wavenumbers and frequencies. Again, ' $x$ ' and dark ' $x$ ' marks indicate the properties corresponding to the maximum growth rates of envelopes and individual peaks, respectively. For the first mode, the most unstable wave (marked with ' $x$ ', at the center of the envelope) has a wavenumber 1.88 (a wavelength of $334 \mathrm{~km}$ ), a frequency of .287 (a period of 32 days) and a phase speed of $12 \mathrm{~cm} / \mathrm{s}$, but the envelope itself moves at group velocity of $29 \mathrm{~cm} / \mathrm{s}$. The most rapidly growing individual peak (marked with dark ' $\mathrm{x}$ ') has a wavelength of $292 \mathrm{~km}$, a period of 20 days and a phase speed of $16 \mathrm{~cm} / \mathrm{s}$. The corresponding envelope moves at a group velocity of $64 \mathrm{~cm} / \mathrm{s}$. Thus the most rapidly growing wave, compared with the envelope, has higher phase speed and group velocity, shorter wavelength and period.

Figure 5.11 compares the properties of individual peaks predicted by the three theories as a function of wavenumber. This is to prepare for the comparisons in Section 5.6 where the group velocity is not a proper variable because of the continuous forcing. Only the first mode from each theory has been plotted. The upper and lower panels show the phase speeds and growth rates respectively. As we can see from the both panels, the three theories give clearly distinguishable pattern of properties as function of wavenumber. Pulse instability gives the largest maximum growth rate, whereas the spatial instability the lowest.

\subsection{A Two-Layer QG Numerical Model}

\subsubsection{Model Description}

The model here differs from Phillips' model used in Chapter 2 in two aspects. The first is the inclusion of topography, in this case a sloping bottom. The second is the nonuniform velocity profiles used here. It is a QG two-layer inviscid channel model. The stream function in each layer is independent of depth. Again, $n=1$ 

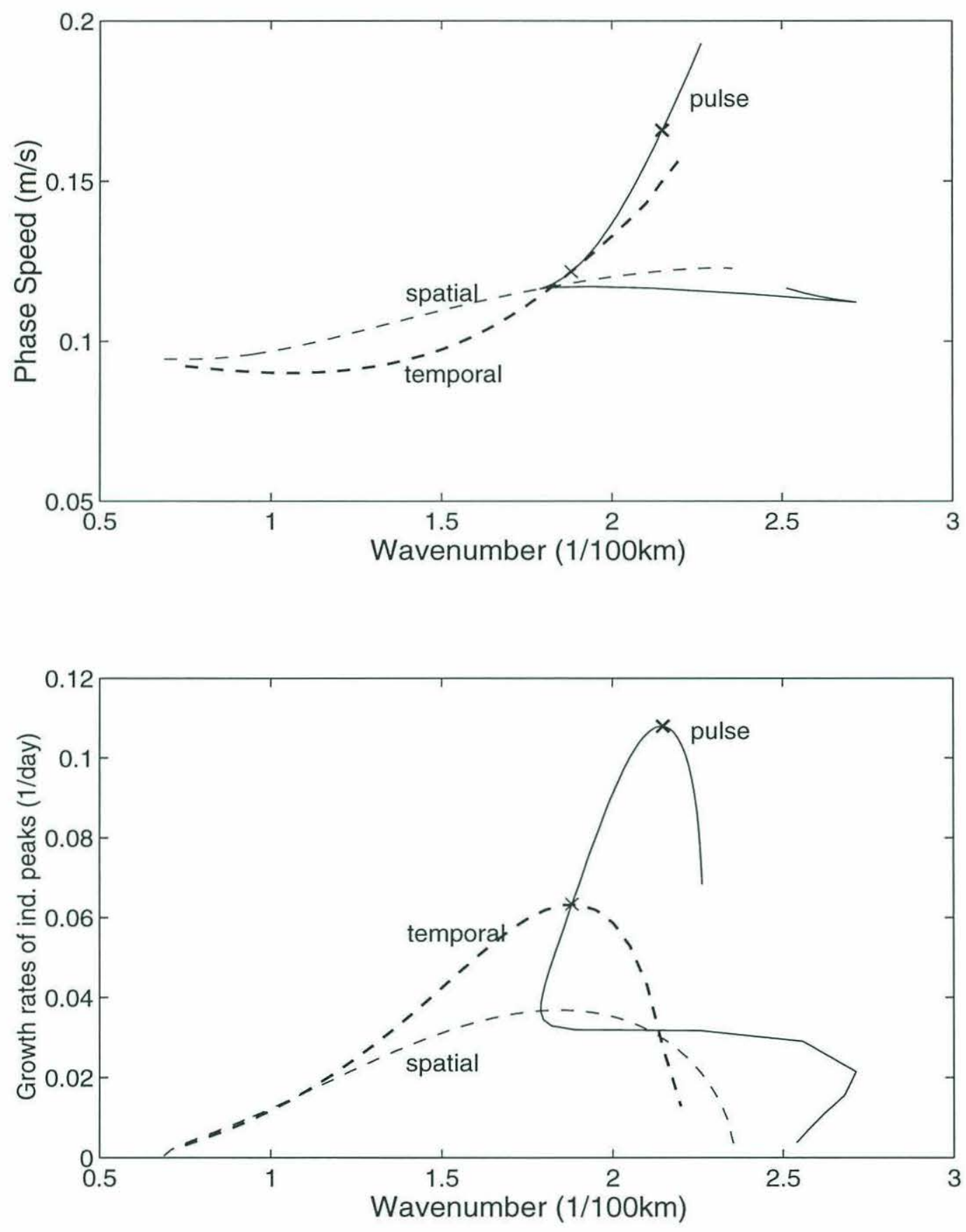

Figure 5.11: Comparison among growth rates of individual peaks predicted by temporal, spatial and pulse instabilities. Only the primary modes from the theories are plotted. 
indicates the upper layer, and $n=2$ the lower one. Based on layer models described in Pedlosky (1986, Sec. 6.16), the equation of motion can be written

$$
\begin{array}{r}
\left\{\frac{\partial}{\partial t}+\frac{\partial}{\partial x} \psi_{1} \frac{\partial}{\partial y}-\frac{\partial}{\partial y} \psi_{1} \frac{\partial}{\partial x}\right\}\left\{\nabla^{2} \psi_{1}-F_{1}\left(\psi_{1}-\psi_{2}\right)+\beta y\right\}=0 \\
\left\{\frac{\partial}{\partial t}+\frac{\partial}{\partial x} \psi_{2} \frac{\partial}{\partial y}-\frac{\partial}{\partial y} \psi_{2} \frac{\partial}{\partial x}\right\}\left\{\nabla^{2} \psi_{2}-F_{2}\left(\psi_{2}-\psi_{1}\right)+\beta y+\eta_{B}\right\}=0
\end{array}
$$

where $\beta$ is the nondimensional gradient of planetary vorticity and $\eta_{B}$ the topography. $F_{1}$, and $F_{2}$ are Froude numbers on each layer.

I separate Equations (5.4.1a-b) into a nonuniform and purely zonal basic flow $U_{n}$ with streamfunction $\Psi_{n}(y)$ and a disturbance stream $\psi_{n}$,

$$
\psi_{n}=\Psi_{n}(y)+\phi_{n}(x, y, t)
$$

leading to

$$
\left[\frac{\partial}{\partial t}+U_{n} \frac{\partial}{\partial x}\right] q_{n}+\frac{\partial}{\partial x} \phi_{n} \frac{\partial}{\partial y} \Pi_{n}+\left[\frac{\partial}{\partial x} \phi_{n} \frac{\partial}{\partial y} q_{n}-\frac{\partial}{\partial y} \phi_{n} \frac{\partial}{\partial y} q_{n}\right]=0
$$

where $q_{n}$ is the perturbation potential vorticity,

$$
q_{n}=\nabla^{2} \phi_{n}-F_{n}(-1)^{n}\left(\phi_{2}-\phi_{1}\right)
$$

and $\frac{\partial}{\partial y} \Pi_{n}$ is the potential vorticity gradient of the basic state,

$$
\begin{array}{r}
\frac{\partial}{\partial y} \Pi_{1}=\beta-\frac{\partial^{2}}{\partial y^{2}} U_{1}-F_{1}\left(U_{2}-U_{1}\right) \\
\frac{\partial}{\partial y} \Pi_{2}=\beta-\frac{\partial^{2}}{\partial y^{2}} U_{2}-F_{2}\left(U_{1}-U_{2}\right)+\beta_{B}
\end{array}
$$

where $\beta_{B}$ is the bottom topographic $\beta$ parameter.

Linearizing and adding forcing gives

$$
\left[\frac{\partial}{\partial t}+U_{n} \frac{\partial}{\partial x}\right] q_{n}+\frac{\partial}{\partial x} \phi_{n} \frac{\partial}{\partial y} \Pi_{n}=F(x, y, t)=G_{n}(x, y) f(t)
$$


where $F(x, y, t)=G_{n}(x, y) f(t)$ is the forcing function. $G_{n}(x, y)$ has the form of

$$
G_{n}(x, y)=B_{n} e^{\left\{-\left(x-x_{n}\right)^{2} / L_{f x}^{2}-\left(y-\frac{L_{y}}{2}\right)^{2} / L_{f y}^{2}\right\}}
$$

The forcing is introduced at a particular location around $\left(x_{n}, \frac{L_{y}}{2}\right)$ with Gaussian distribution. $L_{y}$ is the width of the channel; $320 \mathrm{~km}$ is used. The forcing is therefore concentrated in the center of the channel and the jet. $L_{f x}$ and $L_{f y}$ are the forcing scales in $x$ and $y$ directions respectively. The time dependent part $f(t)$ will specified in Section 5.6

The lateral boundary conditions are

$$
\frac{\partial}{\partial x} \phi_{n}=0 \quad y=0, L_{y}
$$

\section{Pulse Disturbance}

In the experiments concerning evolution of a pulse disturbance, I set $F(x, y, t)$ in Equation (5.4.6) to be 0 and $\phi_{n}=G_{n}(x, y)$ as described in Equation (5.4.7) at $t=0 . A_{1}$ is chosen to be $4 A_{2}$ and is offset by $50 \mathrm{~km}$ to the west arbitrarily, i.e., $x_{1}=x_{2}-50$. For convenience, $x_{1}$ is chosen to be 0 and hence the initial pulse in the upper layer is generated at the origin of the along stream coordinate. $L_{f} x$ is $60 \mathrm{~km}$ and $L_{f} y$ is $30 \mathrm{~km}$. The upper panel in Figure 5.12 shows the initial condition. The $x$ axis was labeled with downstream distance. The lower panel shows a general picture of the developed disturbance after some time.

In this numerical experiment, I use an FFT in $\mathrm{x}$ direction and sine transform in y direction to solve Equation (5.4.6). The sine transform automatically satisfies the boundary conditions in Equation (5.4.8). I use leapfrog time stepping combined with Euler time stepping. An Euler time step is performed every fiftieth time step to remove the computational instability which occurs when leapfrog timestepping is used. 

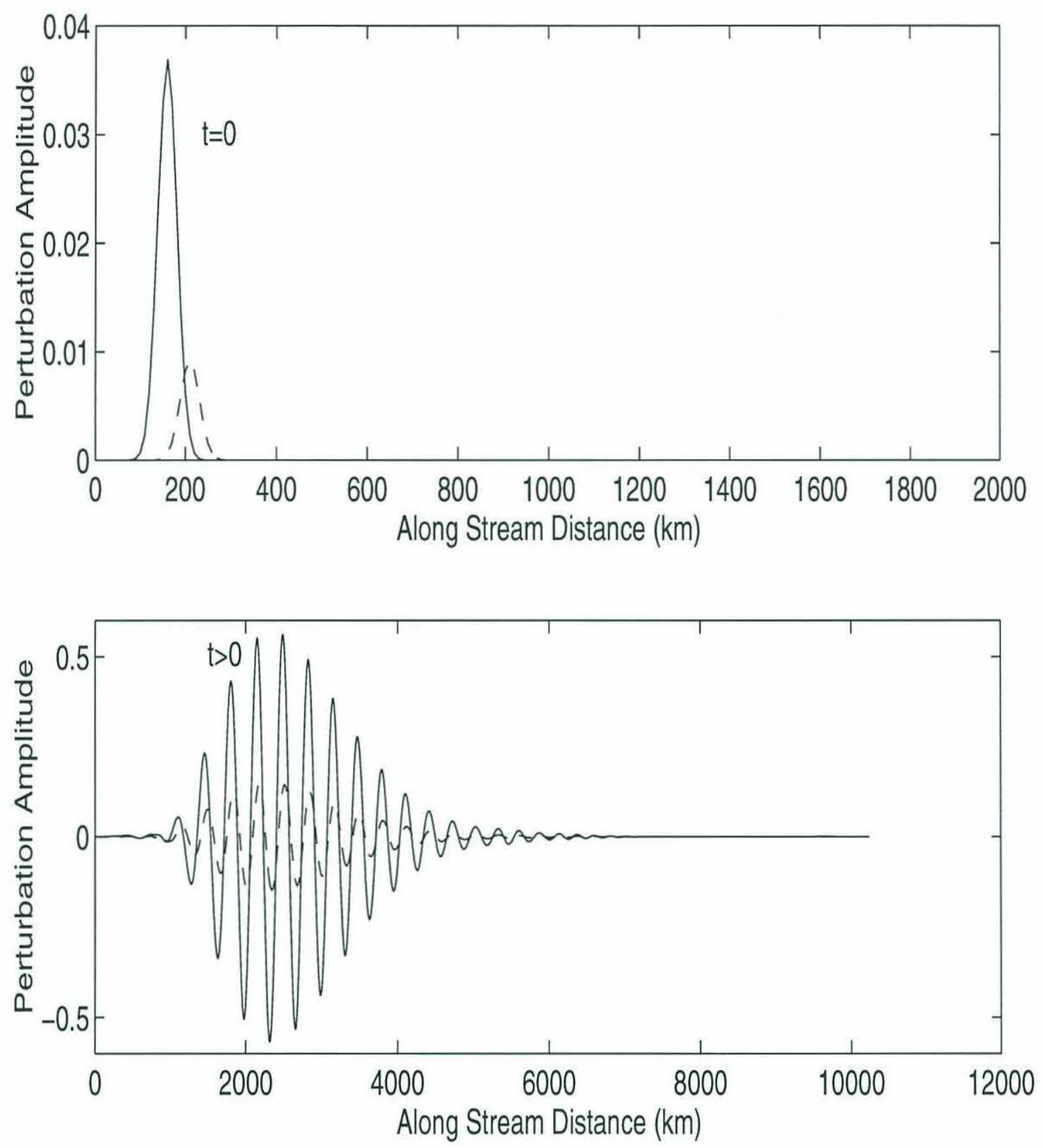

Figure 5.12: Pulse instability. Upper panel: the initial condition used. Lower panel: a typical well developed wave packet. Solid and dashed curves represent the streamfunctions along the center of the jet in the upper and lower layers respectively. 
In the pulse disturbance case, I will use a model domain of $L_{x}=10240 \mathrm{~km}$ and $L_{y}=320 \mathrm{~km}$. Because the model is periodic in $\mathrm{x}$ direction, the numerical run will be stopped when the developed wave packet wraps around the domain. The model resolution is $\Delta x=\Delta y=10 \mathrm{~km}$ and the time step $\Delta t=2000$ seconds.

The numerical model also includes friction which is necessary to keep the numerical run stable. The friction has the form $r_{1} \nabla^{2} \phi_{n}+r_{2} \nabla^{6} \phi_{n}$, where $r_{1}$ and $r_{2}$ are friction coefficients and are minimized as not to affect the comparison between analytical and numerical models.

\subsection{Development of a Single Pulse}

With the initial condition shown in the upper panel of Figure 5.12, I have run the model until the leading edge of the unstable packet reentered the upstream end of the periodic channel. At any time, stream functions in the two layers can be plotted as functions of downstream distance, for example; see the lower panel of Figure 5.12. Starting from the figure, we can locate all the crests and troughs, and use a spline fit to determine the envelope of the wave packet. We can also track individual peaks and calculate the corresponding growth rates. The methods to calculate the growth rates of individual peaks and the envelope have been detailed on page 69 in Section 2.5 and will not be repeated here. Figure 5.13 shows the growth rates calculated from the numerical run on 24 th day.

In the upper panel of Figure 5.13, ${ }^{(*)}$ indicates the growth rate of individual peaks from the numerical run. Also plotted in the figure are the three most unstable modes from the analytical model, for the purpose of comparison. ' $\mathrm{x}$ ' and dark ' $\mathrm{x}$ ' marks on the analytical curves correspond to the maximum growth rates of the envelope and individual peaks respectively. The lower panel plots the growth rates of the envelope from the numerical model (solid curve) and from the analytical model 
(labeled in the figure). We can see from both panels that the wave packet is still far from the asymptotic solution.

The analytical modes plotted in the figure include the correction term $-1 / 2 t$ because this term accounts for a significant portion of the maximum envelope growth rate for the time scale shown here. In particular, this portion is $33 \%, 18 \%, 13 \%$ and $8.7 \%$ on day $24,45,61$ and 91 respectively. This correction term $-1 / 2 t$ does not depend on the group velocity and will hence not affect the relation between the envelope and individual peaks. Adding this term makes easier the comparison between the analytical and numerical results.

Figure 5.14 shows the corresponding wavenumbers ( ${ }^{*}$ ') in upper panel) and phase speeds ( ${ }^{(*)}$ in lower panel) of the unstable waves in the wave packet. The three modes of pulse instability from analytical model are also drawn and labeled in the figure. From the upper panel, the wavenumbers from the numerical run appear to agree well with the first analytical mode in a large range of group velocity ( 0.1 to 0.7$)$. This seems to suggest that the wavenumbers are not sensitive to whether asymptotic form of solution has been achieved. When the group velocity is greater than 0.7 , the wavenumbers seem to match the third and second modes. This phenomena can also be seen in the phase speed plot in the lower panel. This is probably because, in that range, the third and second modes have higher envelope growth than that of the first mode. The phase speeds from the numerical run follow the pattern of the analytical modes, but are still far off from those modes.

Figure 5.15 shows growth rates on 45 th day. In a range of $C_{g}$ from 0.1 to 0.48 , the numerical results agree reasonably with analytical counterparts. When $C_{g}$ is greater than 0.6 , the growth rates again seem to follow the second or third analytical modes for the reason mentioned above. This seems to be a persistent feature showing up even on 24th day (see Figure 5.13) and also on the 61st and 91st days shown later. The second and third modes have similar structures in growth rates and it is difficult 

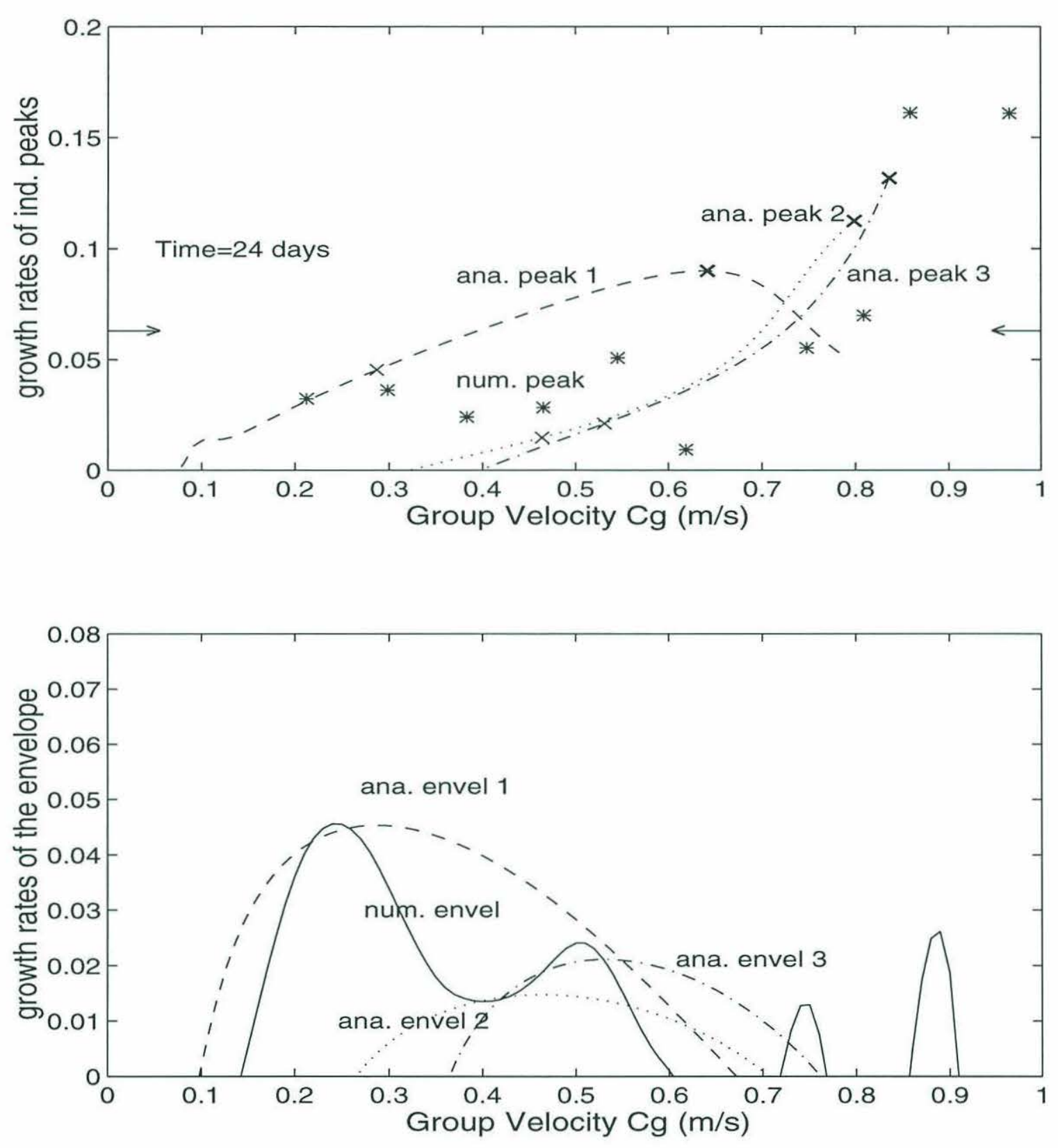

Figure 5.13: Development of a single pulse disturbance. The upper and lower panel represent the growth rates of individual peaks and the envelope, respectively. Also shown are the three most unstable modes (labeled) of pulse instability from the analytical model. Time $=24$ days, always shown on the top panel. The arrows in the upper figure show the maximum growth rate of temporal instability. 

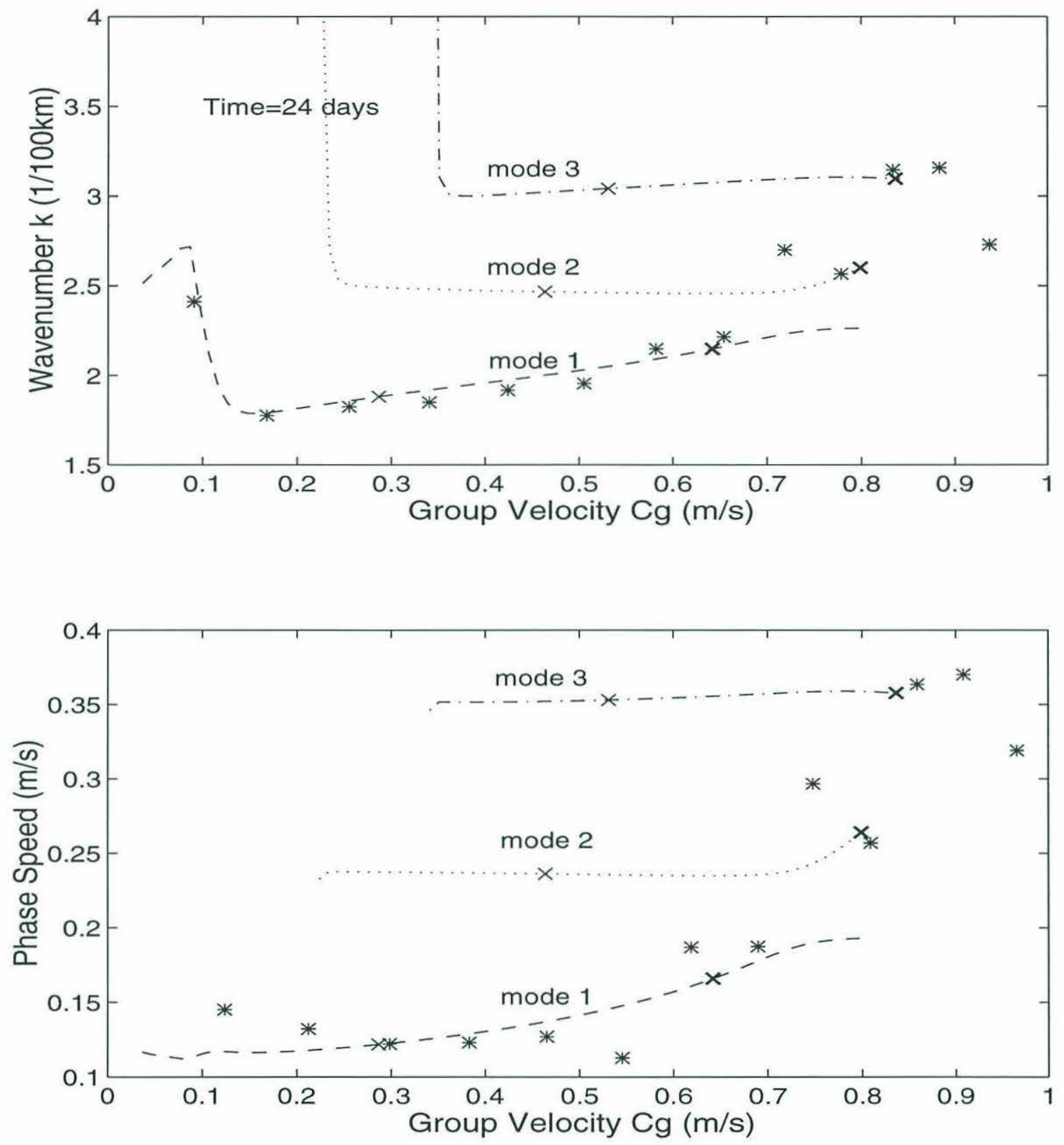

Figure 5.14: Similar to Figure 5.13 except that the upper and lower panel represent the corresponding wavenumbers and phase speeds instead. 
to tell them apart. Between $C_{g}=0.48$ and $C_{g}=0.6$, the first and second modes have comparable envelope growth, and the growth rates of individual peaks in the transition range shift from the first mode to the second.

Figure 5.16 shows the wavenumbers and phase speeds. Again in the range of $C_{g}$ from 0.13 to 0.48 , the numerical results agree reasonably with analytical counterparts. Between $C_{g}=0.48$ and $C_{g}=0.7$, the numerical results seem to follow the first mode and appear feel the influence of the other modes. Beyond $C_{g}=0.75$, three peaks observed are closest to the second mode and then two peaks at leading edge are in the neighborhood of the third mode.

Figure 5.17 converts the horizontal coordinate from $C_{g}$ to downstream distance. As we can see from the figure, the maximum of the envelope is now at $x=1100 \mathrm{~km}$. Since the disturbance was generated at $x=0$, the maximum of the envelope has traveled about $1100 \mathrm{~km}$. Taking the Gulf Stream as a system ranging from $75^{\circ} \mathrm{W}$ to $45^{\circ} \mathrm{W}$, the region covered by Lee and Cornillon's analysis, there appears to be enough space for the pulse instability to reach its asymptotic form. However, the time at which the envelope reaches $70^{\circ} \mathrm{W}$ (about 20 days) is not long enough. The time at which it reaches $65^{\circ} \mathrm{W}$ (about 45 days) is sufficient.

Figure 5.18 shows growth rates on 61 st day. The patterns in the figure are not much different from those in Figure 5.15. In the lower panel, the growth rate of the envelope now has better agreement with analytical model in terms of the shape of the curve. The spike in the trailing edge has been caused by large relative errors due to numerical noise amplified by the spline interpolation. The range of $C_{g}$ where the first mode can explain the numerical results well has expanded slightly from ( 0.13 $0.48)$ to $(0.120 .54)$. This also shows up in the top panel. The fastest growing wave associated with the first mode now is at $C_{g}=0.54$, whereas it was 0.48 on 45 th day. The patterns in the leading edge do not change much between the two figures. 

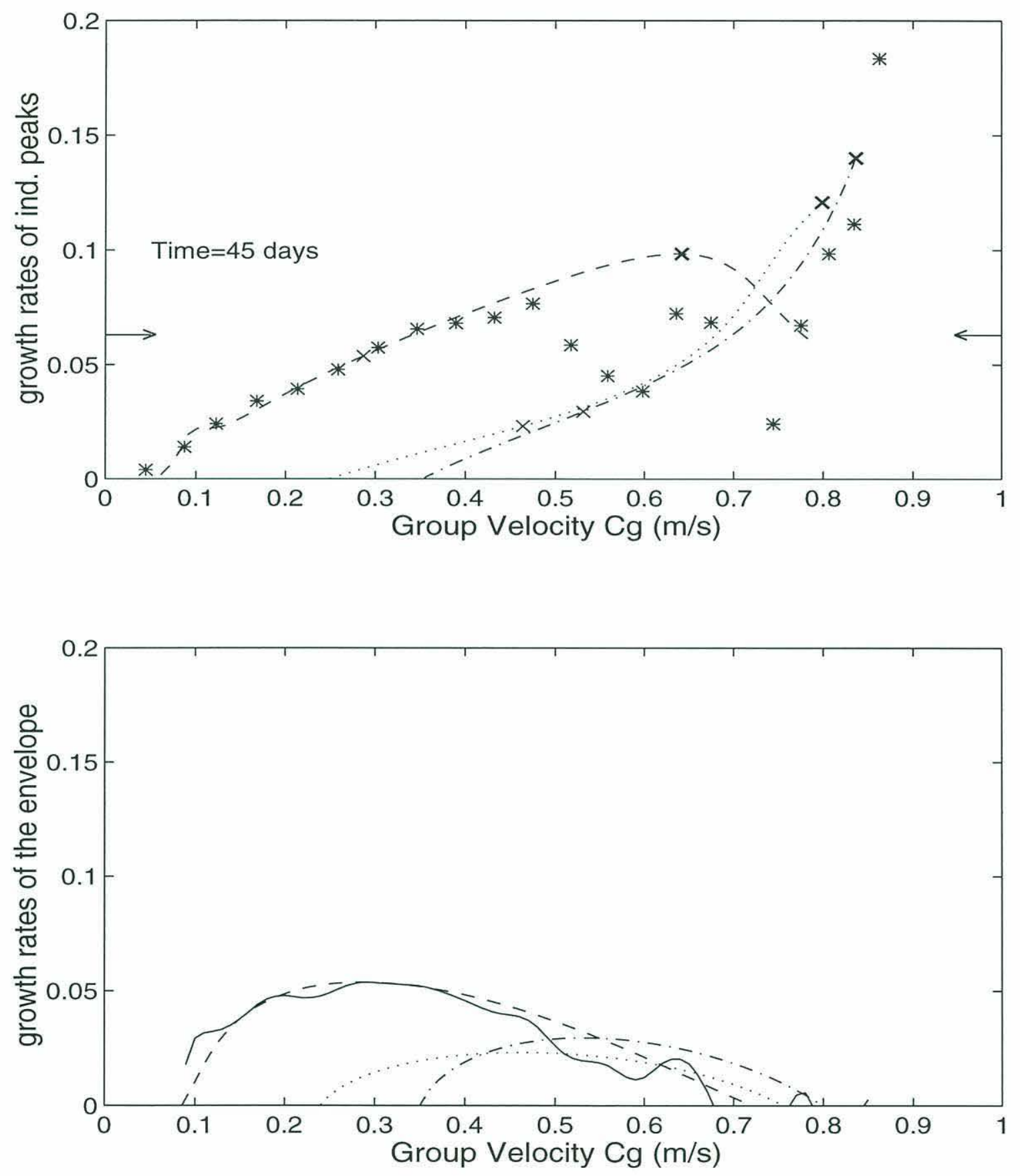

Figure 5.15: As in Figure 5.13 but Time $=45$ days. 

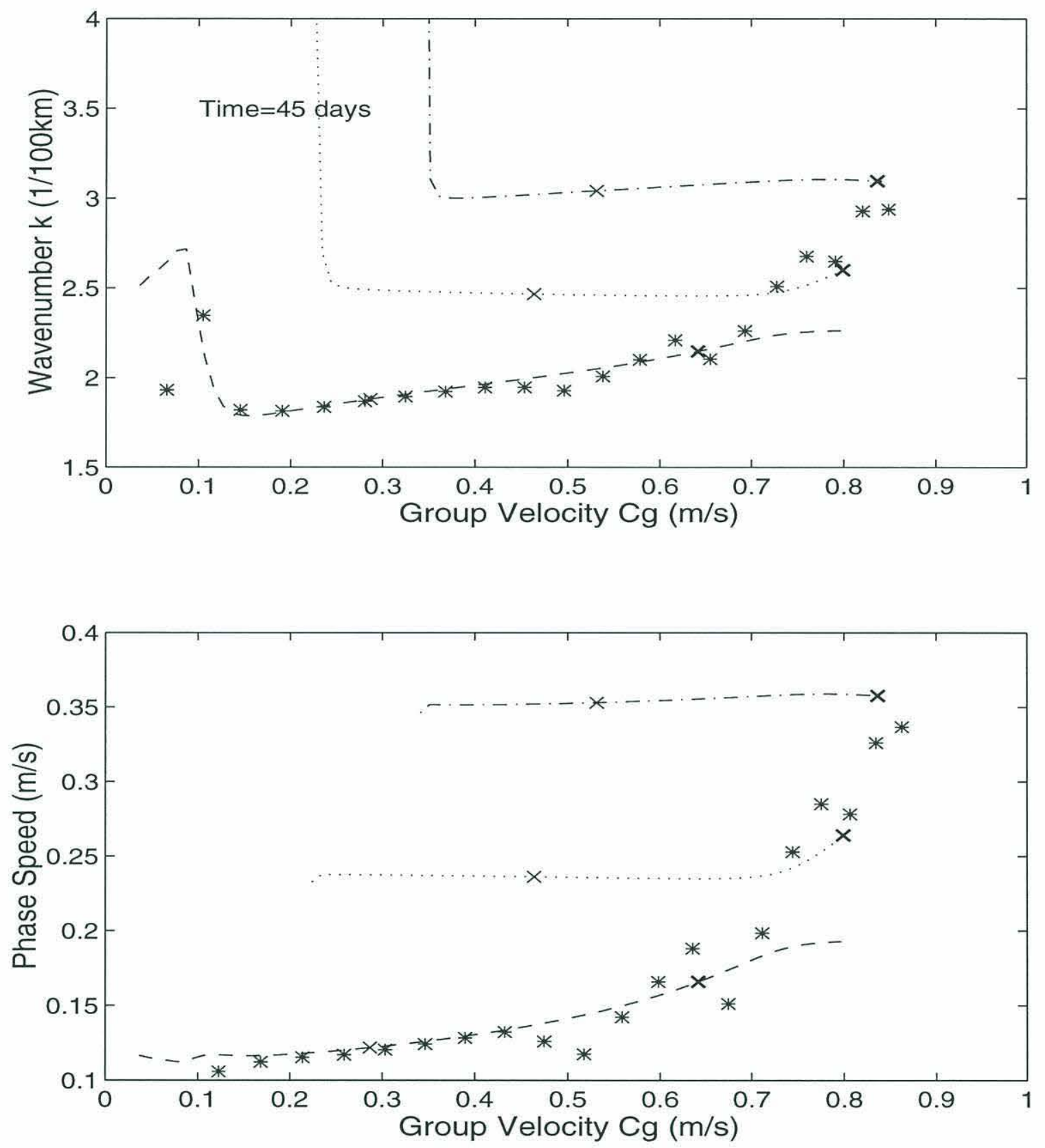

Figure 5.16: As in Figure 5.14 but Time $=45$ days. 

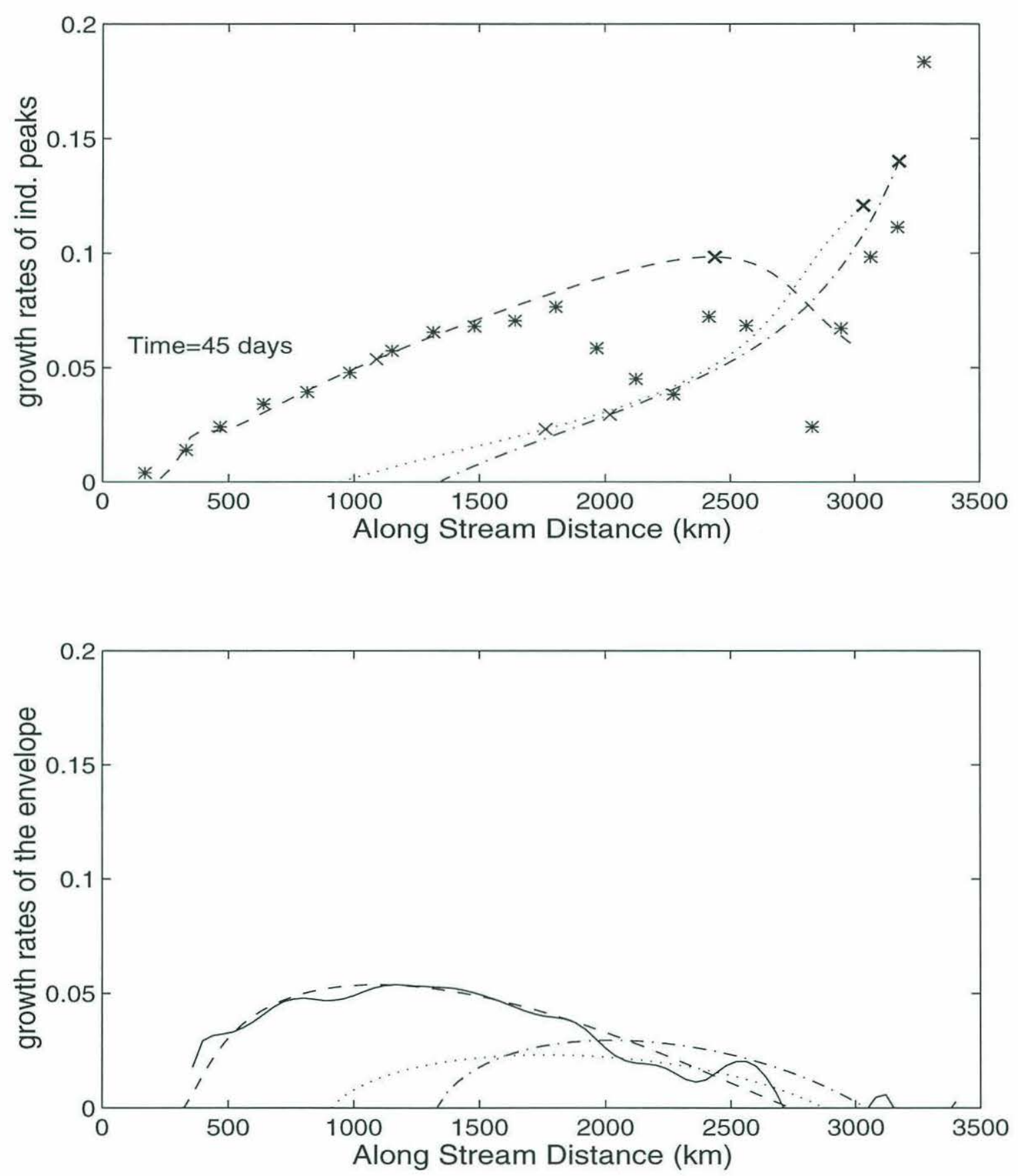

Figure 5.17: As in Figure 5.15 except the horizontal coordinate is the downstream distance. 

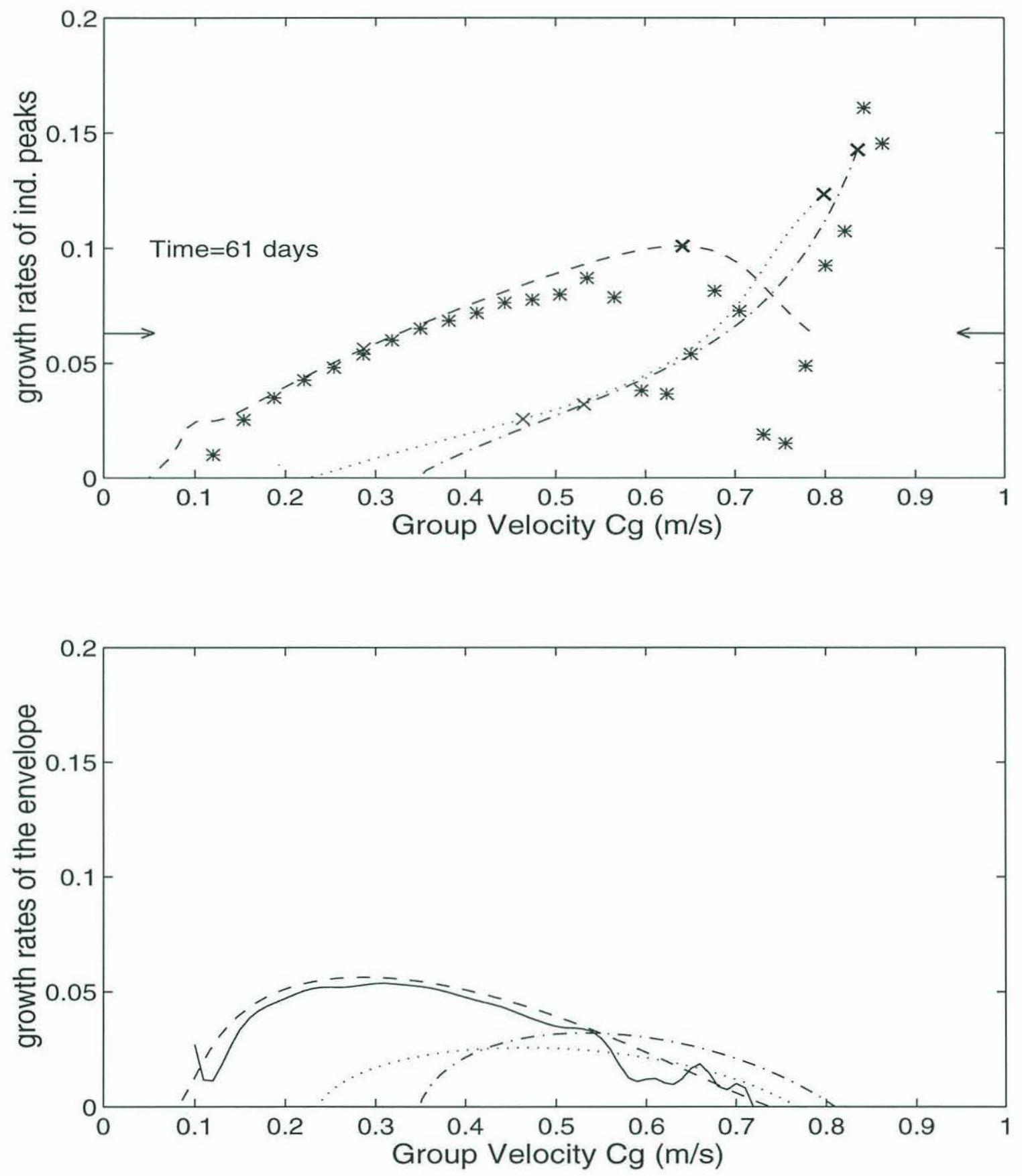

Figure 5.18: As in Figure 5.13 but Time=61 days. 

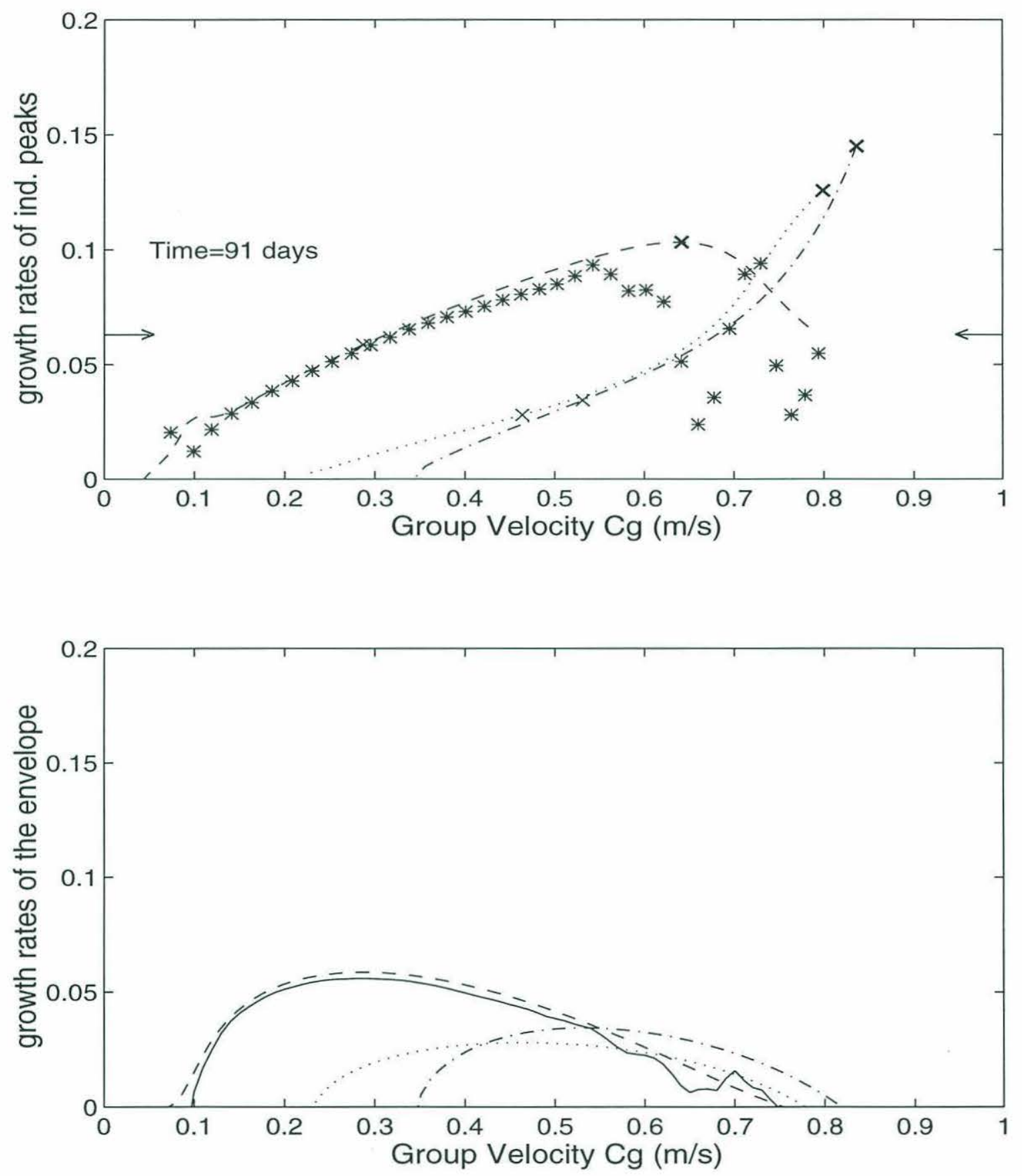

Figure 5.19: As in Figure 5.13 but Time=91 days. 

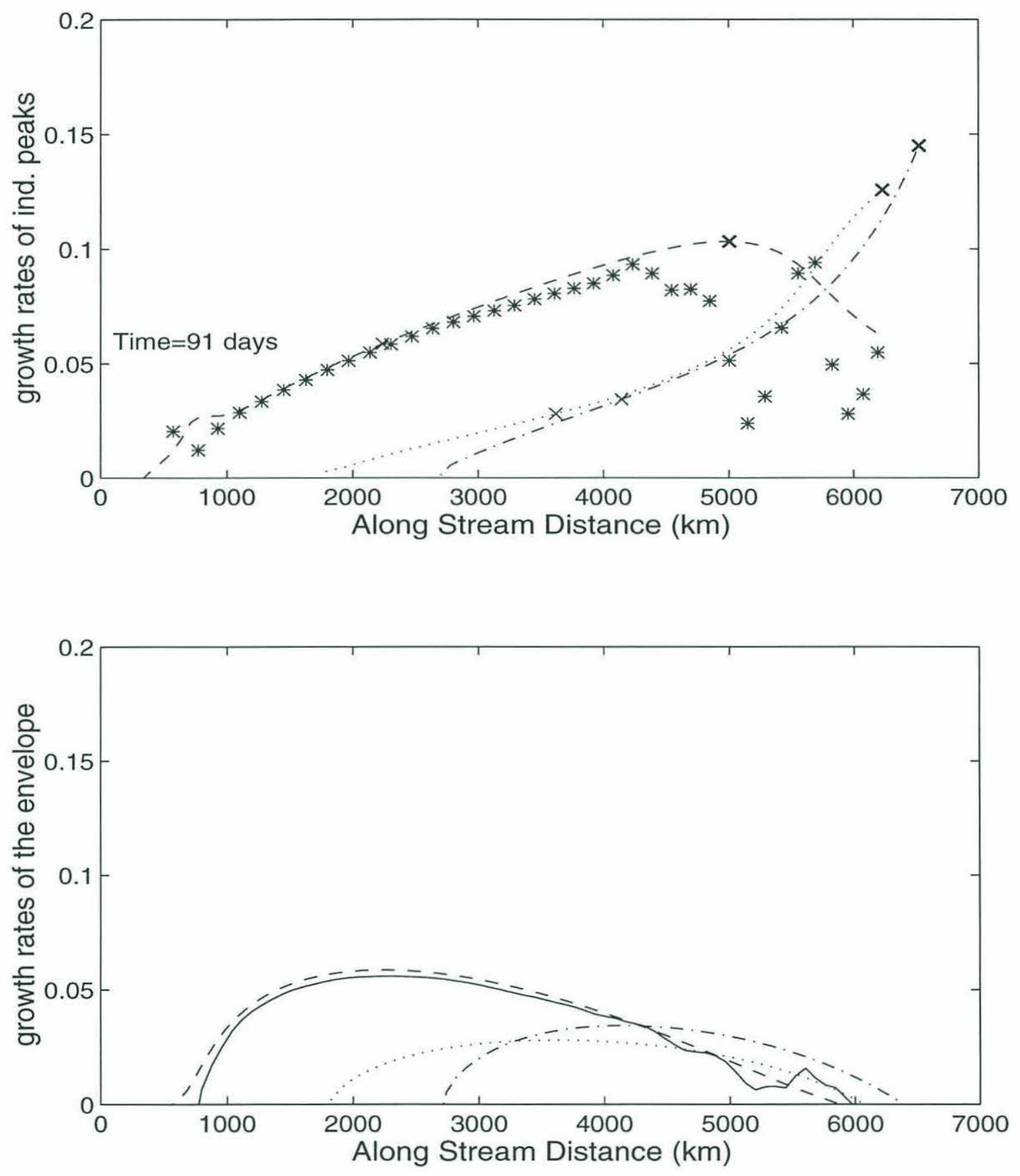

Figure 5.20: As in Figure 5.19 except the horizontal coordinate is the downstream distance. 

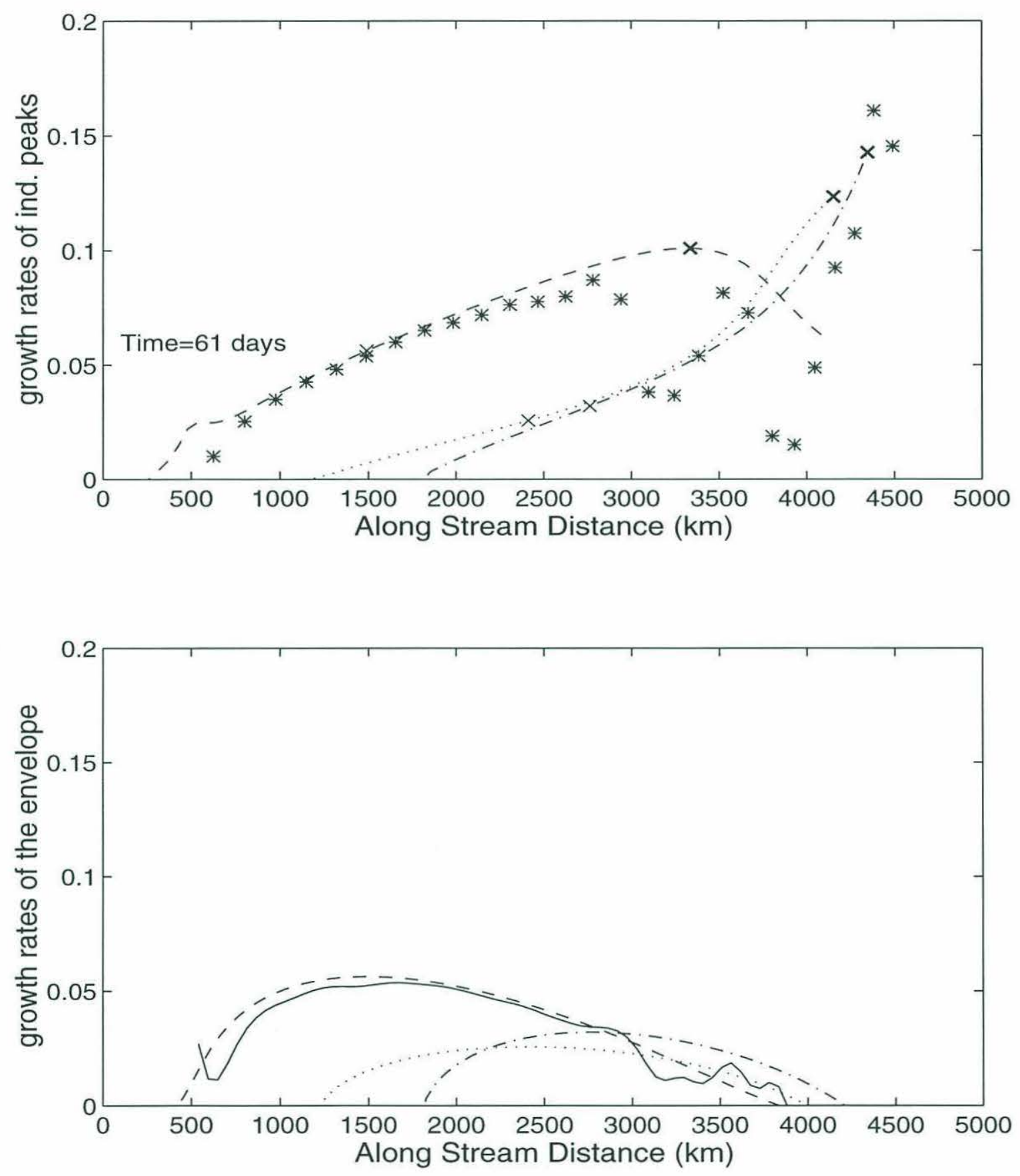

Figure 5.21: As in Figure 5.13 but Time $=61$ days. 

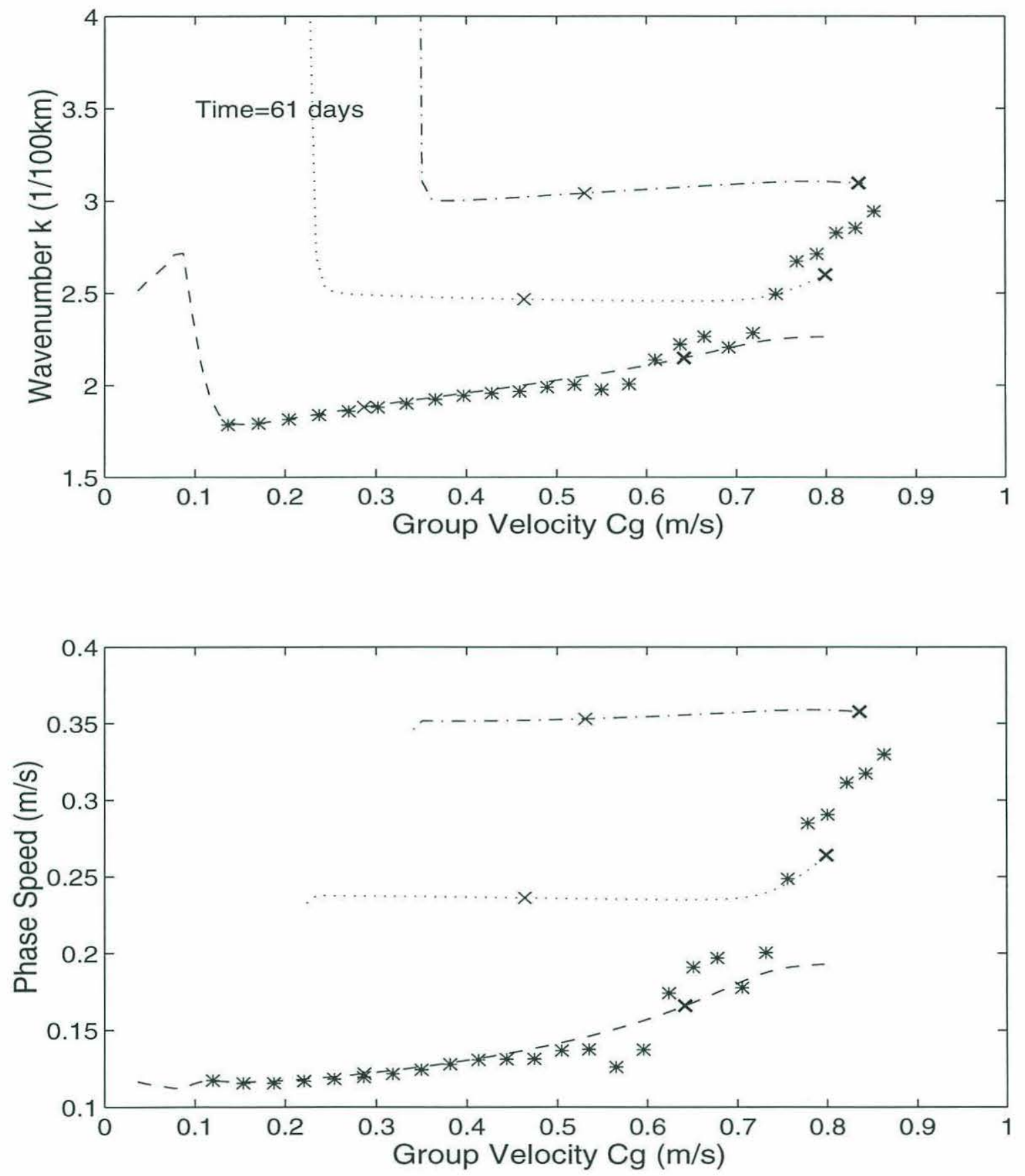

Figure 5.22: As in Figure 5.14 but Time $=61$ days. 
Figure 5.19 shows growth rates on 91 st day. The patterns in the figure are very close to those in Figure 5.18, suggesting that the development of wave packet has finished its transient stage by the 61 st day. The agreement of the growth rates between numerical and analytical models improve slightly but not significantly. This suggests the remaining difference is mostly the effect of friction. The slight improvement in the agreement is probably due to other $O(1 / t)$ contribution other than the correction term $-1 / 2 t$ taken account already. In both figures, the fastest growing peaks occur slightly to the left of $C_{g}=0.55$, which is the point where the envelope growth of first and second mode are the same. This suggests that the fastest growing peak associated with the first mode can only be realized in the region where its envelope growth dominates. This fastest growing peak has slightly lower group velocity, wavenumber and phase speed than that predicted from the first mode of the analytical model. I did not include this term in the demonstration of pulse instablity in Chapter 2 because the time there was chosen to be large.

Note that in the top panel, the fast growing waves associated with the second or the third mode are not picked because I have set a threshold in software to pick only crests or troughs which have amplitudes at least $1 \%$ of the maximum amplitude in the model. As the time increases, the difference between the maximum envelope growth rate in the model and the envelope growth rates in the leading edge has amplified the ratios in amplitudes. Therefore the small amplitude but fast growing waves in the leading edge become 'unobservable' with our predetermined threshold. This may have practical implication in the Gulf Stream observations where the very fast growing but small amplitude waves are unlikely to be observed due to the resolution limits in tracking techniques, for example, errors in pulling out north wall positions.

Figure 5.20 converts the horizontal coordinate from $C_{g}$ to downstream distance. As we can see from the figure, the maximum of the envelope is now at around $x=2200 \mathrm{~km}$. However, the fastest growing wave associated with first mode occurs 
near $x=4200 \mathrm{~km}$; this is beyond the domain of the Gulf Stream. Figure 5.21 shows that on the 61st day, the fastest growing wave associated with first mode occurs near $x=2800 \mathrm{~km}$. Again taking the Gulf Stream as a system ranging from $75^{\circ} \mathrm{W}$ to $45^{\circ} \mathrm{W}$, as covered by Lee and Cornillon's analysis, this fastest growing wave is still in the domain. This fastest growing wave is much more likely to be picked by observational techniques because it has much larger amplitude than the fastest growing wave associated with the second mode in the leading edge for the reason I mentioned above. Therefore I take the fastest growing wave associated with first mode as the plausible fastest growing wave in the numerical model. The fastest growing wave in the following analysis refers to this plausible fastest growing wave.

In the derivation of the growth rate of the envelope in Section 2.3.3, I have assumed that when $\omega(k)$ have branch points, we can avoid going around these points in some way when we integrate to get asymptotic solution. It is difficult to prove this assumption mathematically, in particular in such a complicated model. However, the reasonable agreement with numerical results demonstrates that this is a sensible assumption in this model.

In summary, it takes about 45 days for a single pulse disturbance to develop close to its asymptotic form. In that period the wave packet has moved downstream $1100 \mathrm{~km}$ to about $65^{\circ} \mathrm{W}$. This is still in the range where the Gulf Stream system has been intensively observed. Hence there appears to be enough space for the pulse instability to occur in the Gulf Stream. In about two months, the development reaches its equilibrium stage. On 61st day, the growth rate of the fastest growing wave has a growth rate $0.086 /$ day, which is $60 \%$ higher than the maximum envelope growth $0.053 /$ day. The former has a wavelength, phase speed and period of $(310 \mathrm{~km}$, $14 \mathrm{~km} /$ day, 22 days), and latter (336km, 12km/day, 29 days). 
The following table summarizes the differences between the most unstable wave and the fastest growing wave from our analytical and numerical models, and that described by Lee and Cornillon (1996b).

\begin{tabular}{|l|c|c|c|c|c|c|}
\hline & \multicolumn{3}{|c|}{ most energetic wave } & \multicolumn{3}{c|}{ fastest growing wave } \\
\cline { 2 - 7 } & $\mathrm{L}(\mathrm{km})$ & $\omega_{i}(1 /$ day $)$ & $\mathrm{T}($ day $)$ & $\mathrm{L}$ & $\nu_{i}(1 /$ day $)$ & $\mathrm{T}$ \\
\hline Lee and Cornillon & $427 \pm 70$ & .032 & 46 & 350 & .063 & 40 \\
\hline SW6 & 363 & .044 & 48 & 339 & .105 & 37 \\
\hline QG6 & 349 & .063 & 44 & 293 & .151 & 22 \\
\hline QG2 analyt. & 334 & .063 & 32 & 292 & .108 & 20 \\
\hline QG2 num. 61st day & 336 & .054 & 29 & 310 & .086 & 22 \\
\hline QG2 num. 45th day & 338 & .052 & 29 & 326 & .077 & 23 \\
\hline
\end{tabular}

To make comparison easier, I scale all quantities of the fastest growing wave by the quantities of the most energetic wave to get

\begin{tabular}{|l|c|c|c|c|c|c|}
\hline & \multicolumn{3}{|c|}{ most energetic wave } & \multicolumn{3}{c|}{ fastest growing wave } \\
\cline { 2 - 7 } & $\mathrm{L}(\mathrm{km})$ & $\omega_{i}(1 /$ day $)$ & T (day) & L ratio & $\nu_{i}$ ratio & T ratio \\
\hline Lee and Cornillon & $427 \pm 70$ & .032 & 46 & 0.82 & 2.0 & .87 \\
\hline SW6 & 363 & .044 & 48 & 0.93 & 2.4 & .77 \\
\hline QG6 & 349 & .063 & 44 & 0.84 & 2.4 & .50 \\
\hline QG2 analyt. & 334 & .063 & 32 & 0.87 & 1.7 & .63 \\
\hline QG2 num. 61st day & 336 & .054 & 29 & 0.92 & 1.6 & .76 \\
\hline QG2 num. 45th day & 338 & .052 & 29 & 0.96 & 1.5 & .79 \\
\hline
\end{tabular}

From the above table, it appears that the QG two-layer analytical and numerical model have comparable ratios of quantities between the fastest growing waves and most energetic waves despite the fact that the maximum growth rate in the numerical model is $18 \%$ lower than its analytical counterpart on 45 th day or $14 \%$ on 61 st day. This difference is mainly due to the $O(1 / t)$ contribution neglected in the analytical growth rate. When $t$ is as short as 45 days or 61 days, the main features of pulse instability already show up clearly in the numerical model. This suggests that the analytical results of SW6 and QG6 may also be carried over to their corresponding numerical models. 


\subsection{Continuously Forced Problem}

To have a forcing function with characteristics as close to the real situation as possible, I will use the displacement of North wall of the Gulf Stream at 200km downstream from Cape Hatteras. The data was digitized by Lee and Cornillon (1996a,b) from sea surface temperature (SST) images obtained from the Advanced Very High Resolution Radiometer (AVHRR), as described in Section 4.4. The reason I did not use the displacement of North wall at Cape Hatteras as the forcing function is that the displacement there is small and has the same order of magnitude as the errors from observations and digitizing processes (digitization error 4.8km, Gangopadhyay, 1990).

Figure 5.23 shows a portion of the displacement of North wall at $200 \mathrm{~km}$ downstream as function of time. ' $x$ 's indicate the digitized position available in two day interval and the solid curve is the interpolation of the position into higher resolution for the numerical runs. The distribution of forcing in space is described in Equation (5.4.7). The along stream coordinate is such chosen that the forcing is centered at the origin. The model is only forced in the upper layer.

Because of the large contrast of amplitudes at the ends of the periodic domain, a sponge layer does not work well in this model. If the damping in the sponge layer is too strong, the large amplitude wave near the downstream end of the domain will be reflected back to the domain and cause a resonant growing global mode in the domain. If the damping is too weak, a small fraction of the large wave will pass through the sponge layer. This small fraction of the large amplitude wave will, in fact, dominate over the small amplitude forcing upstream and the forcing becomes ineffective. Therefore I will use a very long domain of $20480 \mathrm{~km}$ without a sponge layer in this experiment. The numerical run will be stopped when the large amplitude wave wraps around and reenters the upstream area. I will only analyze the streamfunction in the upper layer, which is compatible with most observational anal- 


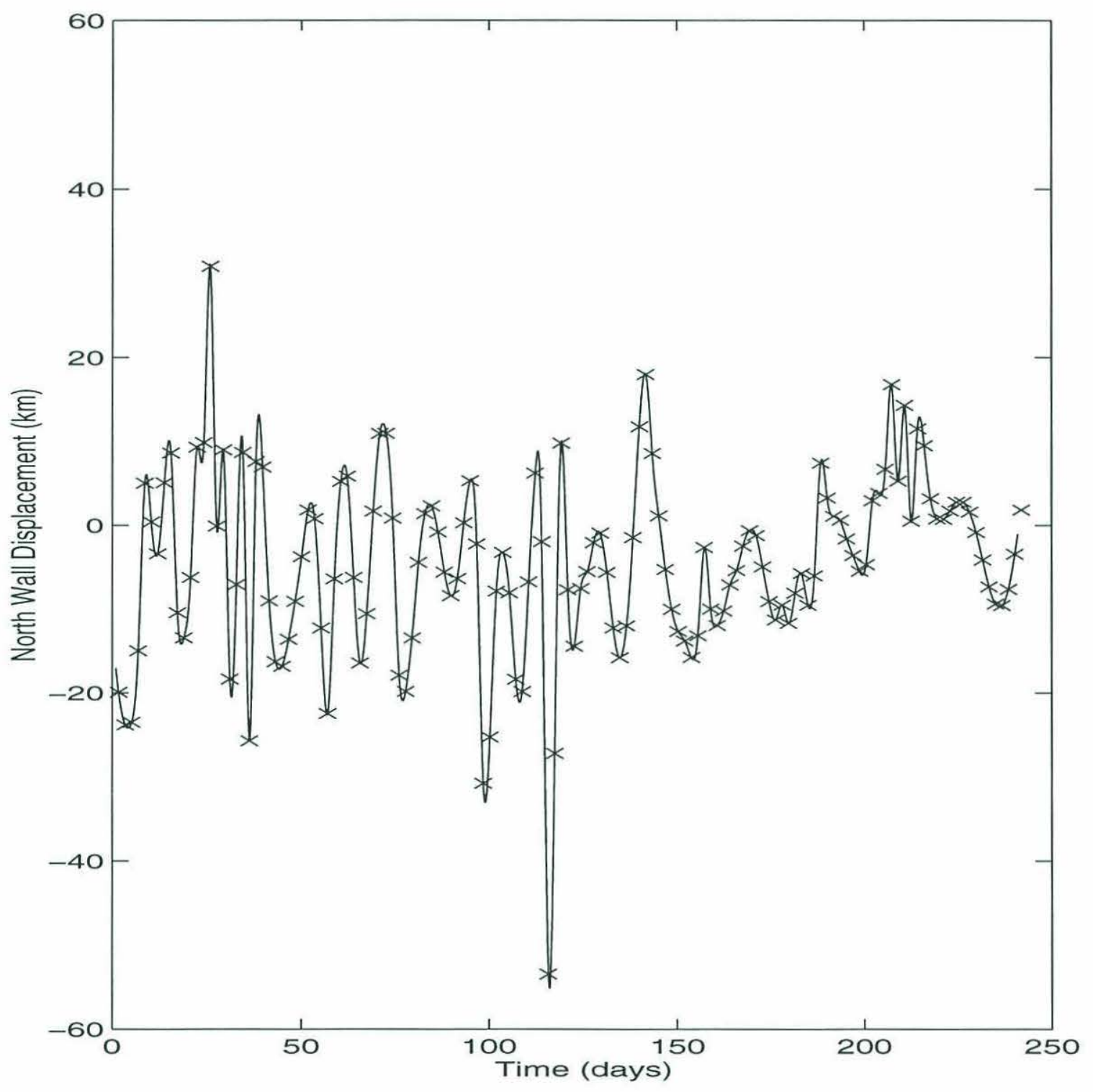

Figure 5.23: The forcing function used. 
yses (for example, Lee and Cornillon, 1996a,b, Halliwell and Mooers, 1983). Due to a numerical difficulty explained later, I can study only the upstream region from $200 \mathrm{~km}$ to $1200 \mathrm{~km}$. The upper panel of Figure 5.24 shows a typical pattern of waves in the continuously forced problem. Also shown is the way I define a half wavelength and the amplitude of the wave. At time $t$, a half wavelength is calculated by measuring the positions of an adjacent crest and trough and the value is assigned to the average position $x$ of the two points. The half wavelength is converted to wavenumber $(k)$ by $k=\pi / L$. A double wave amplitude $(2 A)$ at time $t$ is defined as the difference of the stream functions between the crest and the trough. Such a definition seems to give a reasonable measure for small amplitude crests and troughs even when pairs of crests and troughs are pushed around by relatively large amplitude long waves. The value of this amplitude $A$ is assigned to the average position $x$ of the two points and is also associated with the wavenumber $k$. This method is performed every $2 x 10^{5}$ seconds (2.3 days). The lower panel of Figure 5.24 shows how I track the wave peaks and crests. The streamfunction at $t_{2}$ has been shifted down by 5 units for the convenience of comparison. Corresponding peaks or troughs at time $t_{1}$ and $t_{2}$ are connect by dashed lines. If the position of the pair are $x_{1}$ and $x_{2}$, and amplitudes $A_{1}$ and $A_{2}$, at the time $t_{1}$ and $t_{2}$ respectively, the growth rate and phase speed between $t_{1}$ and $t_{2}$ are defined as $\frac{1}{A_{1}} \frac{A_{2}-A_{1}}{t_{2}-t_{1}}$ and $C_{p h}=\frac{x_{2}-x_{1}}{t_{2}-t_{1}}$, respectively. These values are assigned to the average position $\frac{x_{2}+x_{1}}{2}$ and the average time $\frac{t_{2}+t_{1}}{2}$. To be consistent, the wavelengths and amplitudes of the same pair at $t_{1}$ and $t_{2}$ are also averaged to get the mean value assigned to the time $\frac{t_{2}+t_{1}}{2}$.

The starting time for measurement of wave activity is determined by a control run of a single pulse development as in the previous section. The measurement in the continuously forced problem starts sightly after the maximum of the wave packet in the control run passes the downstream end of the area. This is demonstrated in the upper panel of Figure 5.25. The ending time of the measurement, however, is determined either when the waves wrap around the periodic domain and reenter the 

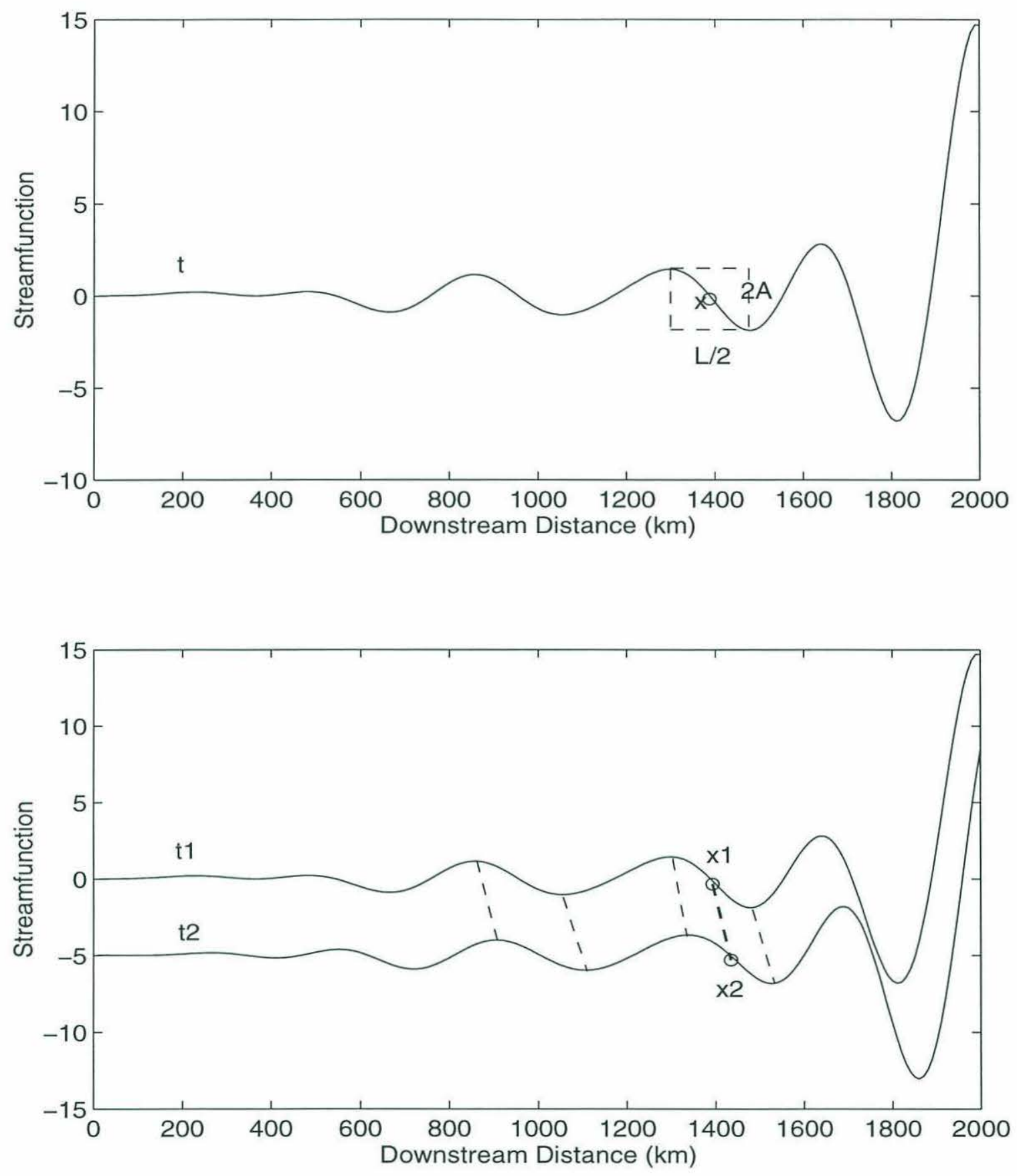

Figure 5.24: Upper panel: a typical pattern of waves (streamfunction in the upper layer) in the continuously forced problem. Also shown is the way I define a half wavelength and the amplitude of the wave. Lower panel: how wave peaks and crests are tracked. The stream function at $t_{2}$ has been shifted down by 5 units for the convenience of comparison. 
upstream end of the study area, or when the limited precision used starts to cause large numerical error. The lower panel of Figure 5.25 shows the latter case. For a given precision, the model will blow up after a certain amount of time when the amplitude contrast in the model becomes very large. This probably is a specific problem of the model due to the use of periodic domain and Fourier expansion. It also happens in the single pulse experiments. Essentially, the problem comes from the use of Fourier series to expand the streamfunction with large amplitude contrast in different regions. When the amplitude contrast is small, the Fourier components will cancel among themselves exactly where the wave packet has not reached, in the case of the single pulse experiments. When the amplitude contrast becomes substantially large (e.g., $10^{6}$ ), however, there will be large relative errors due to inaccuracy in the Fourier transformation (FT) and inverse FT. These errors will grow in time as the amplitude contrast increases. The errors will be larger in the region with smaller amplitude, especially in the upstream region or in the front of the leading edge of the first wave packet caused by the onset of the forcing.

The measurements from different runs, corresponding to different segments of the forcing function, are pooled together for statistics. In the continuously forced problem, we cannot plot quantities such as growth rates and phase speeds as functions of $C_{g}=x / t$. This is because we do not know, when we observe a particular developed wave, the time when its corresponding initial disturbance was generated. Hence in this problem we plot phase speeds and growth rates vs. their corresponding wavenumbers. Figure 5.26 shows such a plot.

Figure 5.26 plots phase speeds (dots in the upper panel) and growth rates (dots in the lower panel) of individual wave peaks in the area from 200 to $1200 \mathrm{~km}$ downstream. Also plotted are the first analytical modes from pulse (solid curve), temporal (dark dashed) and spatial (dashed) instabilities. In the figure, the dots measured from the numerical runs scatter widely, which cannot be explained by any 

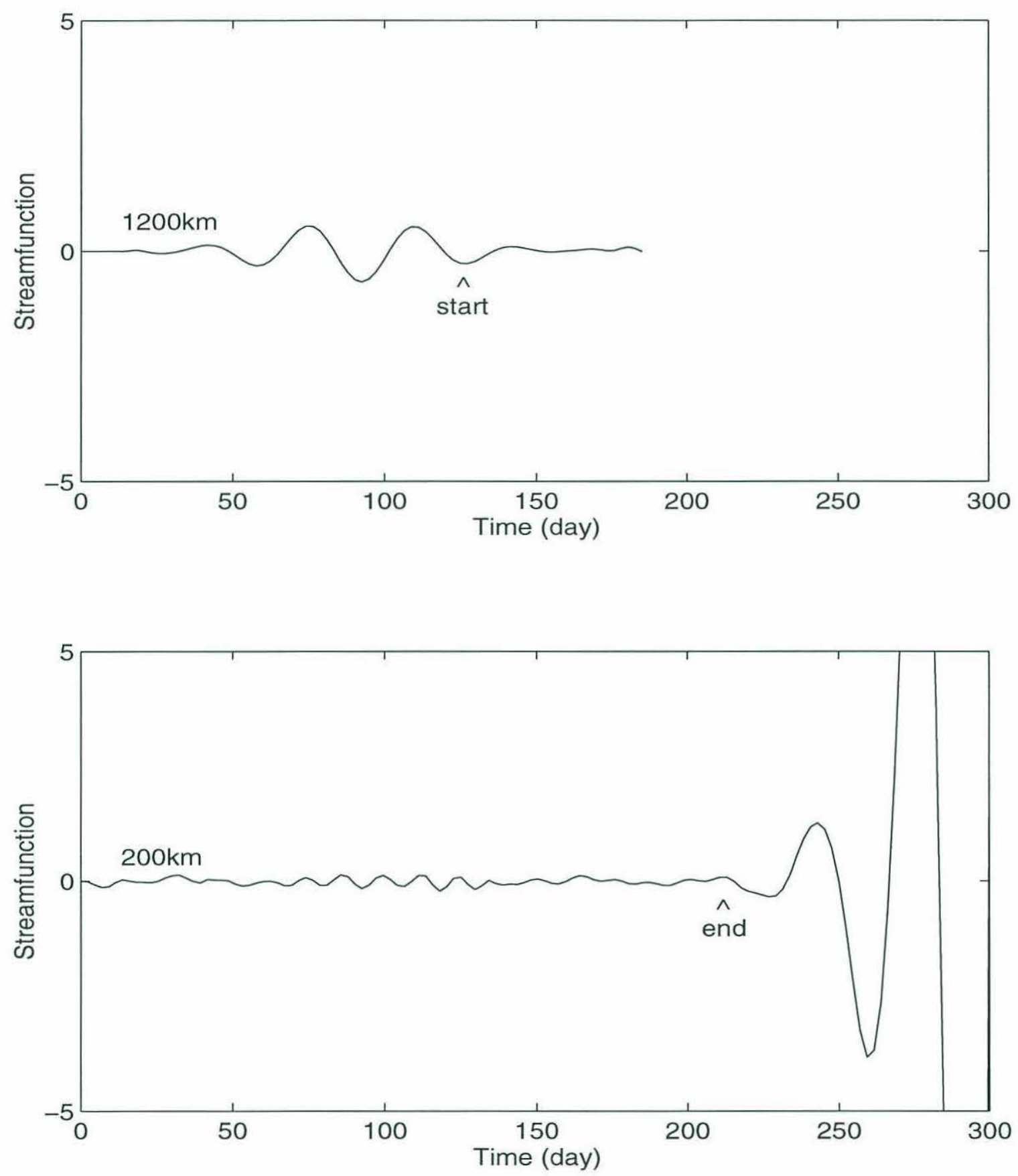

Figure 5.25: A demonstration how the starting and ending times from measurement are determined. Upper panel: time series at $1200 \mathrm{~km}$ (downstream end of the study area) from the single disturbance run. Lower panel: time series at $200 \mathrm{~km}$ (upstream end of the study area) from the continuously forced problem. 
of the three idealized types of instabilities. It is unlikely that the errors arise from measuring positions, calculating wavenumbers and growth rates. Interpolation errors should be about a kilometer spatially and a few percent of maximum amplitudes. In addition, the same method was also used in the single pulse experiment where phase speeds, wavenumbers and growth rates were well-behaved and agreed well with analytical results. It is most likely to be associated with interference of different waves generated by the forcing.

Figure 5.27 shows the least square fit (dark straight line) to the growth rates. The slope is 0.2029 and has a $95 \%$ confidence interval of [0.1637 0.2420]. The slope at the maximum envelope growth from the pulse instability is 0.2449 , slightly higher than the upper bound of numerical interval. This could be explained by the friction used. In the numerical experiments, I used minimal friction to avoid the numerical instability. The friction has the form $r_{1} \nabla^{2} \phi_{n}+r_{2} \nabla^{6} \phi_{n}$, where $r_{1}$ and $r_{2}$ are friction coefficients and has stronger damping effect for short waves. The straight line fit almost passes both of the maximum of the envelope growth and the maximum of individual peak growth. This might be by accident. However, these measurements form a general trend: the short waves tend to grow faster than long waves. This trend seems consistent with the pulse instability theory and cannot be explained by temporal and spatial instabilities.

To quantify this trend, I break the measurements into different wavenumber bins. The interval of the bin is $0.1(1 / 100 \mathrm{~km})$. Sufficient number of measurements in a bin allows calculation of mean and standard deviations (hence error bars). Figure 5.28 shows these averages and errorbars. The averages, indicated by ' 0 ', are connected by dash-dotted curve for convenience of comparison with theories. The errors are so large that we cannot exclude the temporal and spatial instabilities. Only the pulse instability seems to agree with measurements within errors near the maximum growth 

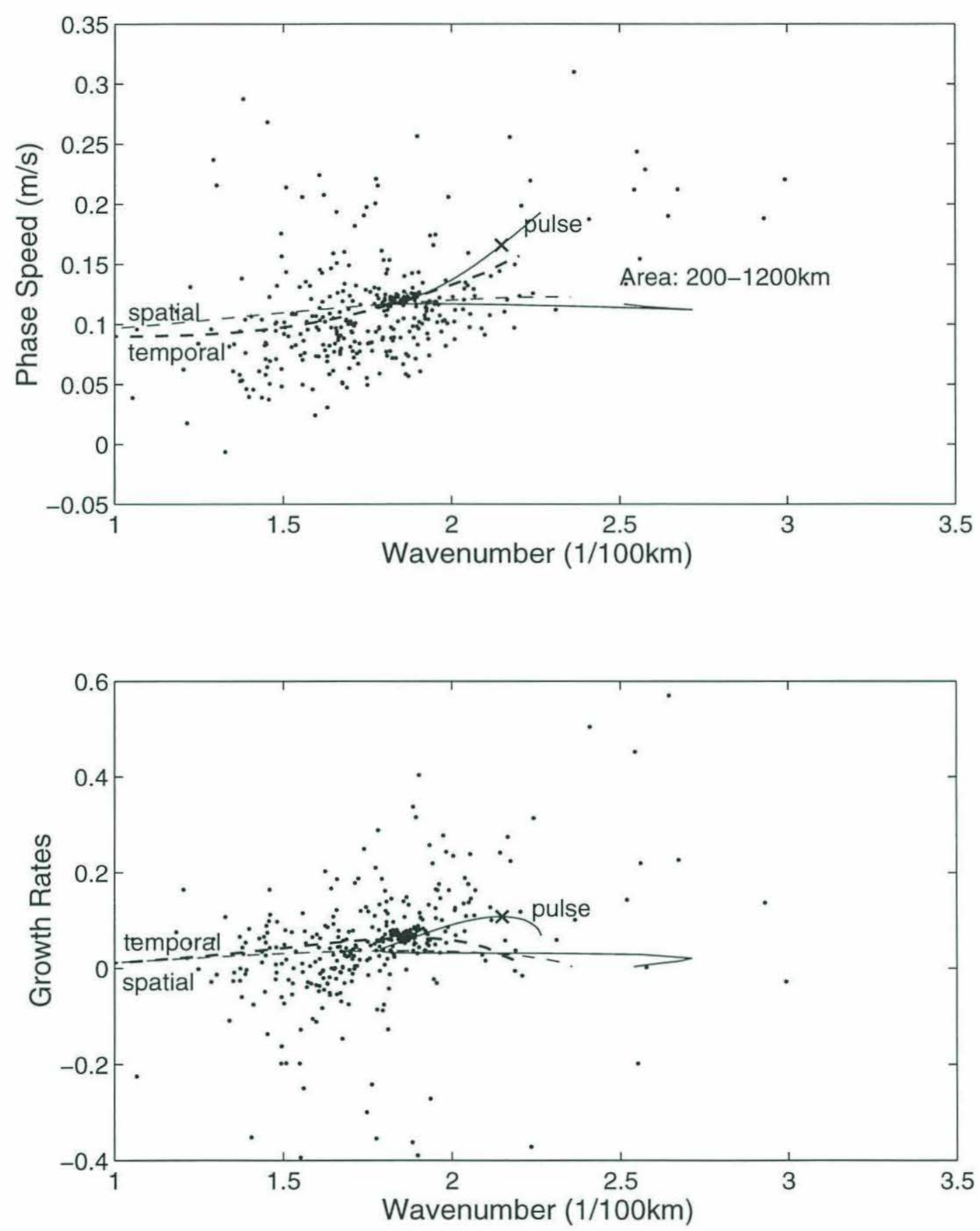

Figure 5.26: Phase speeds (dots, upper panel) and growth rates (dots, lower panel) of individual peaks as functions of wavenumber. Analytical curves: solid: pulse, dashed: spatial, dark dashed: temporal. Area studied: $200-1200 \mathrm{~km}$. 


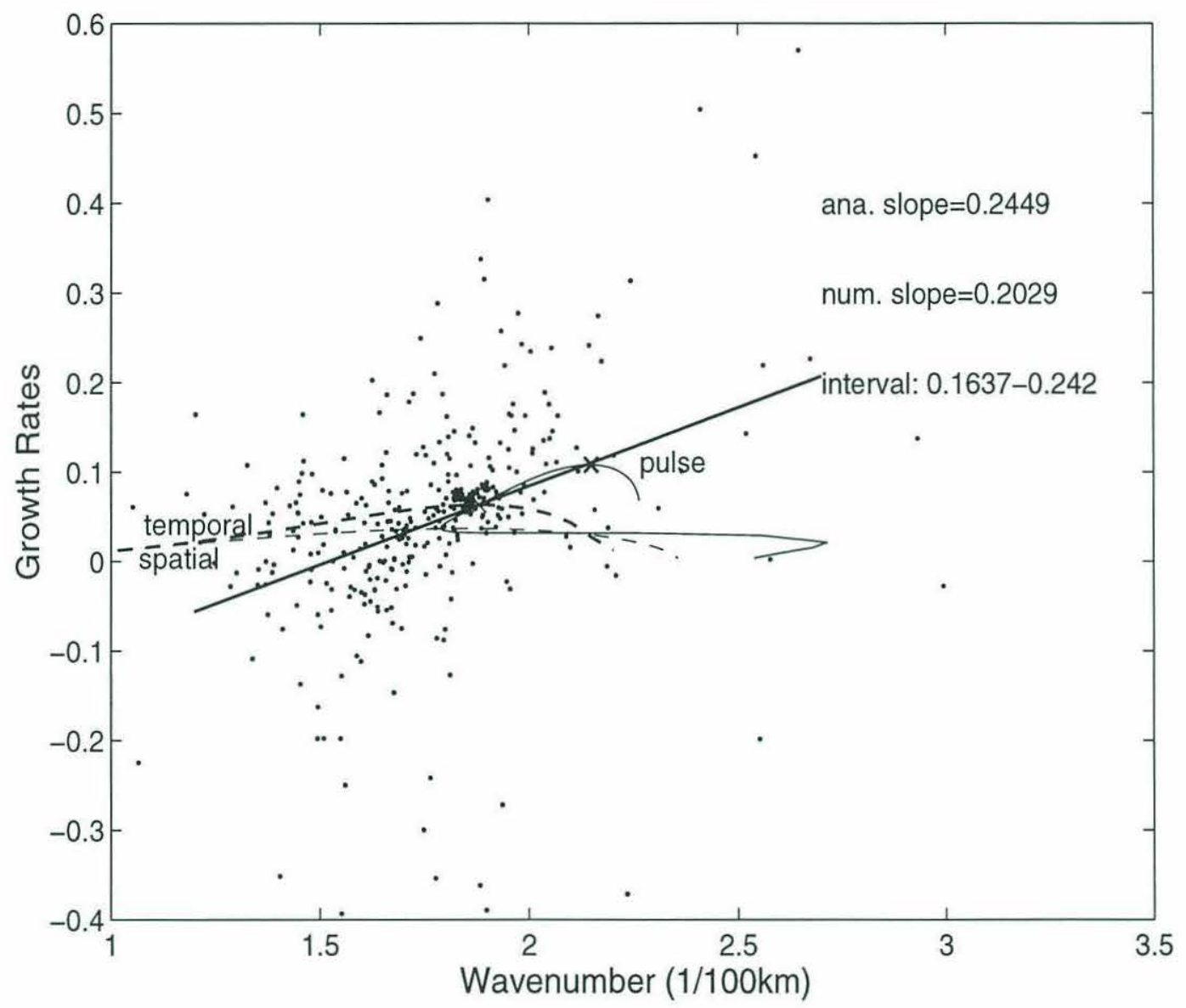

Figure 5.27: As in the lower panel of Figure 5.26. The dark straight line is the least square fit to numerical results. Area studied: $200-1200 \mathrm{~km}$. 


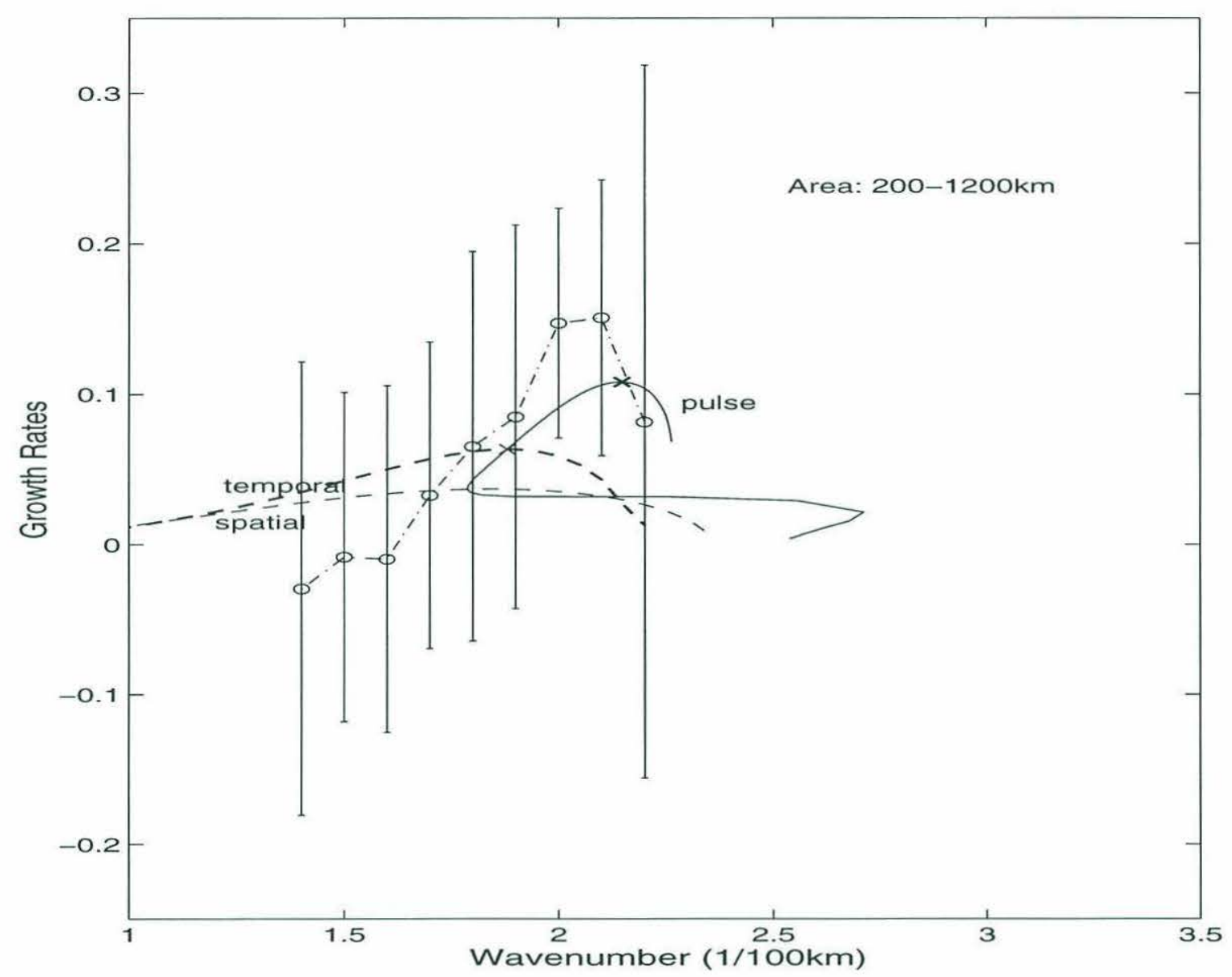

Figure 5.28: Similar to Figure 5.27. Average growth rates ('o') and error bars of individual peaks as functions of wavenumber. Dash-dotted curve connect these averages for the convenience of comparison. Analytical curves: solid: pulse, dashed: spatial, dark dashed: temporal. Area studied: $200-1200 \mathrm{~km}$.

of individual peaks. Pulse instability cannot explain the growth rates (some negative) in the trailing edge.

Figure 5.29 is similar to Figure 5.28 except average phase speeds are shown instead of average growth rates ('o'). The average phase speeds do not seem to fit any of the analytical curves. This is probably due to forcing and interaction between pulses. When the wave amplitudes are small, errors in measuring the positions of peaks will be large because peaks are close to flat. In Figure 5.29, the waves with 


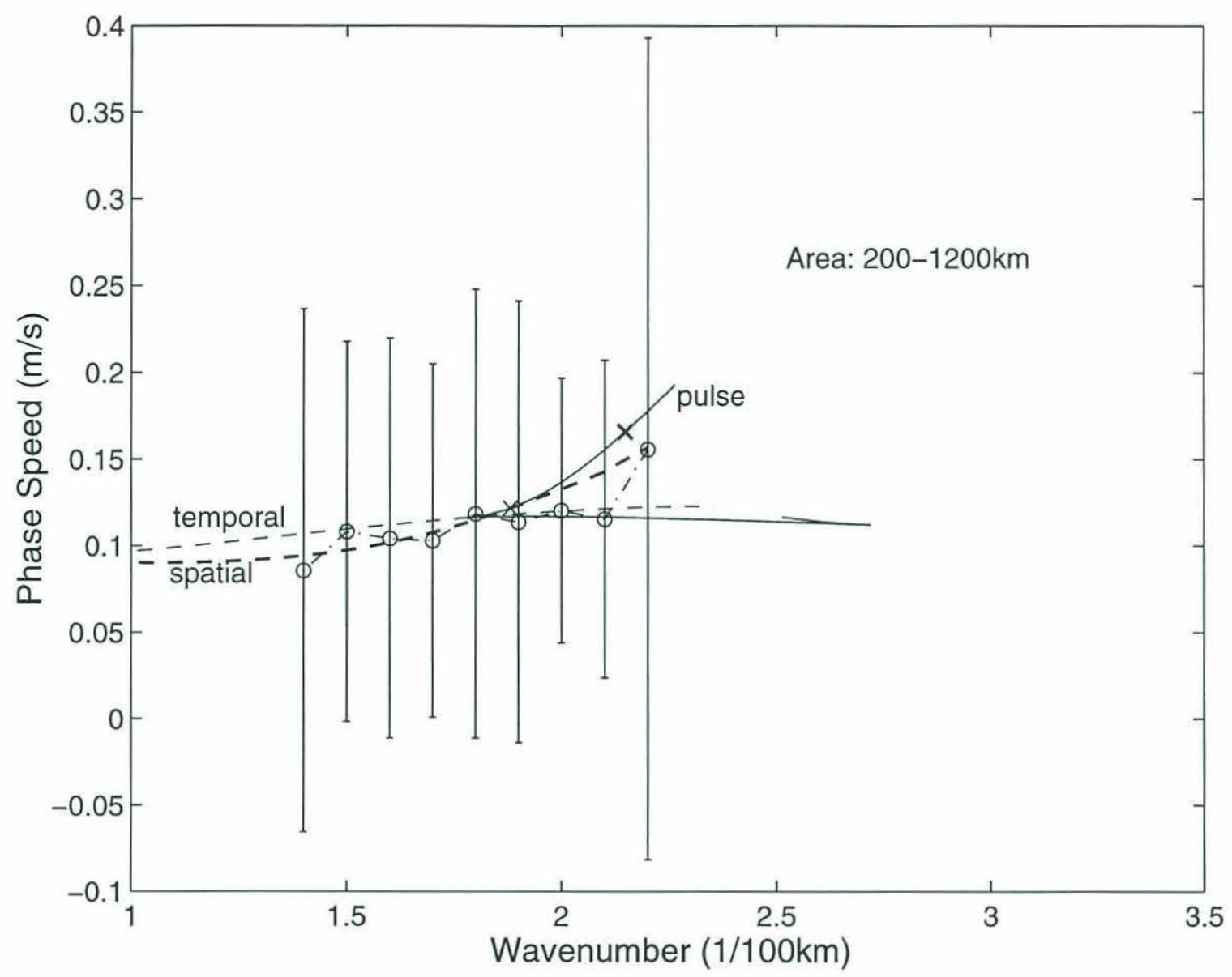

Figure 5.29: Similar to Figure 5.28 except average phase speeds are shown instead of average growth rates ('o'). Analytical curves: solid: pulse, dashed: spatial, dark dashed: temporal. Area studied: $200-1200 \mathrm{~km}$.

wavenumber higher than that of most unstable wave still have tendency to move faster than longer waves.

The upper panel in Figure 5.30 shows a histogram of the probability distribution of growth rates of individual peaks. ' $\mathrm{x}$ ' and ' $\mathrm{o}$ ' indicate the maximum growth rates from temporal and spatial instabilities, respectively. The dark ' $x$ ' is the maximum growth rate of individual peaks predicted by pulse instability. The bins have width of $20 \%$ (0.013) of the maximum temporal growth rate (0.063/day). This growth rate is the most probable growth rate in the area. From the cumulative probability distribution in the lower panel, we can see that a significant portion (40\%) of the 
peaks have growth rates higher than the maximum temporal growth rate, and half of these have growth rates higher than the maximum peak growth predicted by pulse instability. Such large growth rates are probably due to constructive interference between different wave pulses. Similarly, distructive interference may also be the reason for the decaying waves on the left end of the upper panel. One very important implication of the experiment is that if we measure the growth rate of an individual peak in the Gulf Stream, there is a good chance of finding that its growth rate exceeds the maximum growth rate predicted by either temporal or spatial theory.

From the single pulse experiment, we know that it takes about 45 days for a single pulse disturbance to develop close to its asymptotic form. In that period the wave packet has moved downstream $1100 \mathrm{~km}$ to about $65^{\circ} \mathrm{W}$. The area further downstream (1000 to $2000 \mathrm{~km}$ ) is where the asymptotic pulse instability theory is supposed to work better. An attempt was also made to analyze the wave activities in the area. Similar to Figure 5.25, Figure 5.31 shows how to determine the starting and ending times for the measurement. The method is the same for the upstream area. However, as we can see from the figure, the appropriate starting time is determined to be later than appropriate ending time, which leaves no time for measurements. The ending time is limited by the large amplitude contrast and the precision used in the model, as explained in the above analysis in the upstream area, and is almost the same for the upstream and downstream area considered. However, the starting time is delayed substantially in the downstream area for two reasons. First, the wave packet has to travel extra $1000 \mathrm{~km}$ to reach the downstream end of the area, at the group velocity $\left(C_{\operatorname{gmax}}\right)$ corresponding to the maximum of the envelope. Second, the wave packet becomes much longer than it was in the upstream area and it takes much longer time for the whole packet to pass through the downstream area. A solution to the problem is to increase the precision and accuracy of the model, and hence the ending time. However, since we are not able to implement a sponge layer without serious reflection, we need a long domain for the wave packet to travel downstream. 

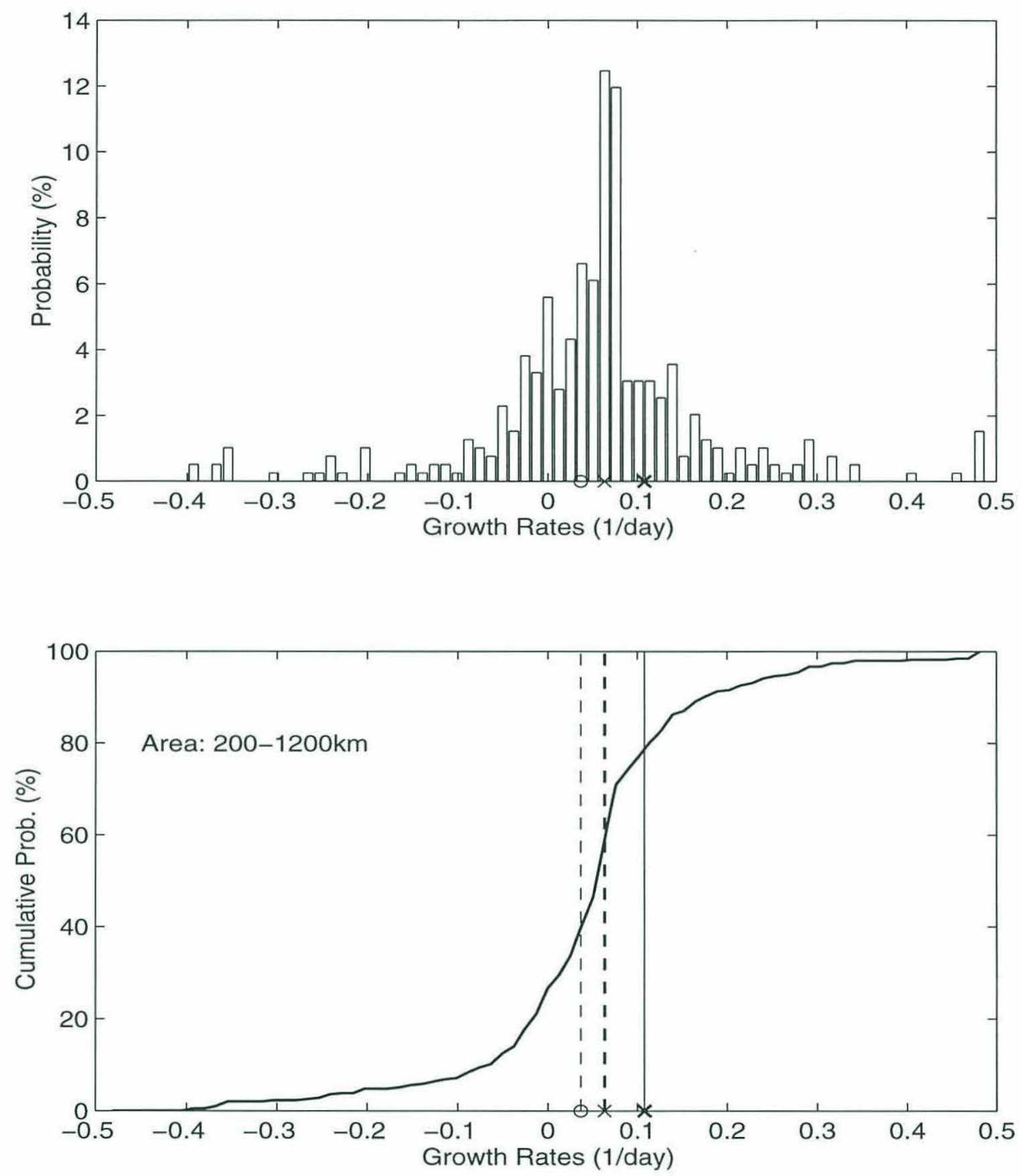

Figure 5.30: Upper panel: a histogram of the probability distribution of growth rates of individual peaks. ' $x$ ' and ' $o$ ' indicate the maximum growth rates from temporal and spatial instabilities, respectively. The dark ' $x$ ' is the maximum growth rate of individual peaks predicted by pulse instability. Lower panel: cumulative probability distribution of growth rates of individual peaks. Vertical lines indicate the maximum growth rates from the three types of theories: solid: pulse, dashed: spatial, dark dashed: temporal. Area studied: $200-1200 \mathrm{~km}$. 
The demands for long domain and high accuracy require more computing resource and I have not been able to obtain sufficient "observations" to give reliable answers in the downstream area.

\subsection{Summary and Discussion}

From the numerical experiments, we have found that it takes about 45 days for a single pulse disturbance to develop close to its asymptotic form. In that period the wave packet has moved downstream $1100 \mathrm{~km}$ to about $65^{\circ} \mathrm{W}$. This is still in the range where the Gulf Stream system has been intensively observed. Hence there appears to be enough space for the pulse instability to occur in the Gulf Stream. The development reaches its equilibrium stage in about two months.

In the continuously forced problem, we have found that the growth rates of individual peaks from the numerical runs scattered widely, in the region from $200 \mathrm{~km}$ to $1200 \mathrm{~km}$ downstream of the forcing location. It is clear that neither of the three idealizations of the instability can explain the pattern in this area. However, the "observations" from the model run do show trends predicted by pulse instability: short waves tend to move and grow faster than long waves. This cannot be predicted from temporal or spatial instability. Unfortunately, due to a numerical difficulty, I have not been able to extend the analysis to the region further downstream, where the pulse instability has been found to work better in the single pulse experiment.

These numerical experiments again support the robust common features of pulse instability we have studied in Chapter 2 to 4 , that the fastest growing wave has higher growth rate, wavenumber and frequency than the most unstable wave.

Xue (1991b) also carried out similar numerical experiments of the Gulf Stream on the South Atlantic Bight in a 3D primitive model. She used both cyclic 

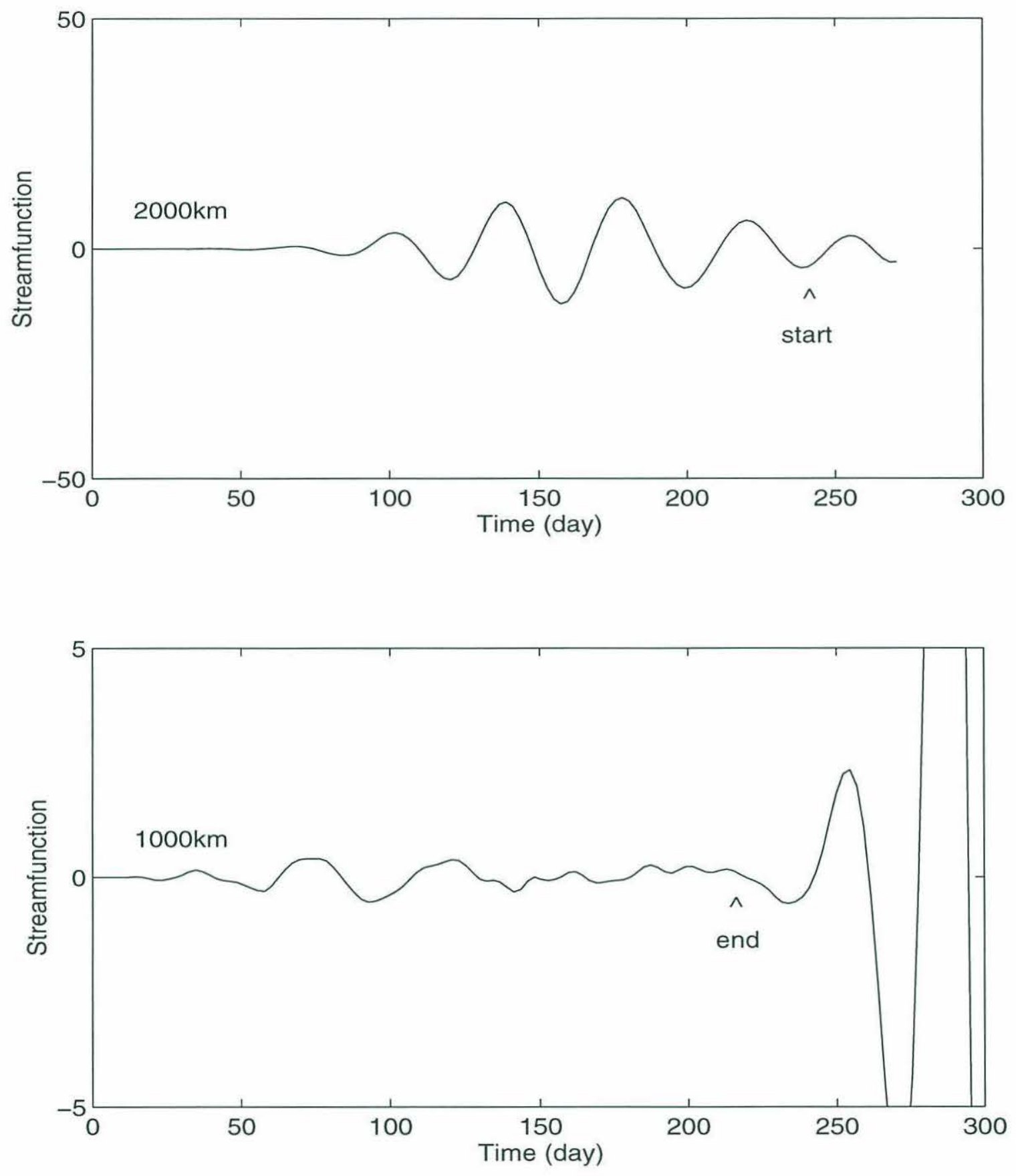

Figure 5.31: A demonstration how the starting and ending times from measurement are determined. Upper panel: time series at $2000 \mathrm{~km}$ (downstream end of the study area) from the single disturbance run. Lower panel: time series at $1000 \mathrm{~km}$ (upstream end of the study area) from the continuously forced problem. 
boundary condition and the gravity wave radiation condition. The former is the same as the periodic domain in this model. In the experiments with cyclic boundary conditions, she used a domain length of $800 \mathrm{~km}$ and the disturbances were generated automatically by numerical noise in the nonlinear model. She only estimated a growth rate of the dominant wave in the model from the change of eddy kinetic energy and did not resolve the full dispersion relation. Since the most unstable waves in her models travel at phase speed of about $40 \mathrm{~km} /$ day, it only takes 20 days for waves to wrap around the periodic domain of $800 \mathrm{~km}$. Hence what she resolved was probably close to temporal instability. In the experiments with the gravity wave radiation boundary condition, the model was forced by an oscillating temperature field at the southern boundary (starting end of the model). The period of forcing (6 days) was chosen based on observed period. The most unstable waves in the model appear to have periods of about 6 days and wavelengths very close to those observed. This is not surprising in light of our discussion in Chapter 2 based on Briggs (1964) work. When the system is forced with constant frequency, spatially instability tends to occur and the dominant wave has the same frequency as the forcing. The result was not analyzed in terms of any of three types of instabilities. Thus it is hard to infer from these simulations which is the most appropriate theory for the Gulf Stream meandering. This thesis work differs from her work in the emphasis on the comparison between temporal, spatial and pulse instabilities. And the continuously forced problem in this chapter uses a more realistic nonperiodic forcing (north wall displacement) obtained from satellite data.

In light of our findings from the continuously forced problem, it appears very difficult to extract analytical dispersion relation based on the numerical measurement, even in this simple model which has many fewer complicating factors than the real Gulf Stream (e.g. no nonlinearity, no downstream variation of basic flow and topography, etc.). However, when we average the growth rates of individual peaks in bins of wavenumbers, the average tend to agree with pulse instability to some ex- 
tent. Hence this experiment suggests that the better way to compare observations with analytical model is to group observational data into bins of wavenumbers before comparison. This in fact was the way Lee and Cornillon (1996b) used to find out the fastest growing wave. In their analysis, they used bins of wavelengths ( $50 \mathrm{~km}$ interval) to group the observational data. Because the difference in wavelengths between the most energetic and the fastest growing waves is of the order of $50 \mathrm{~km}$, we suggest use of bins with smaller interval, e.g., $15 \mathrm{~km}$ or $20 \mathrm{~km}$. However there is trade-off between the resolution and the number of data points in a bin, and a balance has to be struck based on the total number of data points.

The continuously forced problem does not support spatial instability. Hence it discourages the way Johns (1988) and Kontoyiannis (1992) compared their model analytical dispersion relations with the observations. They extracted spatial growth rates from EOF analysis and compared them with equivalent or pseudo-equivalent spatial growth rates (ESGR or PESGR) from temporal instability discussed in Chapter 2 and 3. 


\section{Chapter 6}

\section{Summary and Conclusion}

In Chapter 2, we used Phillips' model (two-layer QG) to determine and compare the characteristics of temporal,spatial and pulse instabilities. We then extended the study to a more realistic QG six-layer model with jet structure and topography in Chapter 3. Chapter 4 studied non-quasigeostrophic effect by comparison of a SW six-layer model with the QG six-layer model. In Chapter 5, we used a QG two-layer numerical model to examine which of three idealized types of instabilities is most appropriate in the context of the Gulf Stream given continuous forcing. The conclusions have been made at the end of each main chapter. Here I will organize these conclusions in terms of types of instabilities and important issues addressed. Some results from others will also be cited here to provide a complete context of this study.

\subsection{Analytical Studies}

\section{Temporal Instability}

Briggs (1964) proved that for any system to support unstable waves, its dispersion equation must yield complex solutions with positive imaginary parts for some real $k$, i.e. it is a necessary condition for any unstable waves. This implies that spatial and pulse instabilities only occur when temporal instability also occurs. This furthermore implies that Charney and Stern's necessary condition for temporal instability in geophysical context (1962) is also necessary condition for spatial and pulse instabilities. 


\section{Spatial Instability}

Briggs also shows that not all solutions with the right sign for spatial instability are growing waves. We need to apply Briggs criterion (described in Section 2.3) to distinguish real growing waves from other solutions with the same sign. I have used the criterion to say that the implication in Hogg's (1976) work that spatial instability does not require potential vorticity gradients to change sign is not legitimate. In addition, I have shown that the spatially unstable wave Hogg considered is really better thought of as neutral because of the assumption that phase speed is real. Hence one of his results, that spatially growing waves are bounded by a low wavenumber cut-off which separates them from the temporally growing waves, is not valid.

Michalke (1965) first studied the difference between spatial growth rate (SGR) and pseudo-equivalent spatial growth rate (PESGR). He found that PESGR was $17 \%$ smaller than SGR in a barotropic flow with a hyperbolic tangent velocity profile. In Chapter 2 and 3, I have made comparisons among SGR, PESGR and ESGR (the equivalent spatial growth rate) and examined the validity of Gaster's formula in the Phillips model and the QG six-layer model with relatively realistic jet structure and topography. In the QG six-layer model, we have found that spatial growth rate (SGR) is generally larger by about $30 \%$ than the equivalent spatial growth rate (ESGR) converted from temporal instability. The difference is slightly bigger than that in Phillips model probably due to jet structure or topography. Pseudo-equivalent spatial growth rates (PESGR), transformed from temporal growth rates divided by phase speeds, exceed SGR by $200 \%$ at low frequency and are not a good estimate of $\mathrm{SGR}$ in general.

As seen in both Chapter 2 and 3, real parts of wavenumbers (or wavelengths) corresponding to the most temporally and spatially unstable waves are comparable and not sensitive to mean velocities, velocity shear and $\beta$. 


\section{Pulse Instability}

The maximum of the envelope of the growing disturbance moves at group velocity of the most temporally unstable wave, and grows at the rate of the same wave. The growth rate of an individual peak depends on the location of the peak relative to the maximum of the envelope. Given $C_{g}>C_{p h}$, individual peaks behind the maximum of the envelope grow at a rate smaller than $\omega_{i \max }$. At the leading edge, individual peaks can grow much faster than the peak of the envelope, which makes the envelope move faster than the individual peaks. These general results were first found by Simmons and Hoskins (1979) in atmospheric observation. The formula of growth rate of an individual peak was also given in the paper without a derivation. In Chapter 2, I have provided a derivation of the formula and focused on the behaviors of individual peaks. In particular, when the growth rate of an individual peak is plotted as a function of its amplitude, it decreases as the amplitude increases. It has been demonstrated in this work that the relation between the growth rate of an individual peak and its amplitude is an intrinsic property and an important feature of pulse instability. Thus if one follows a single peak and notices a decrease in the growth rate, one cannot infer that increasing amplitude (or nonlinearity) was responsible for reducing the growth rate. This is also demonstrated in Chapter 3 with a more realistic dispersion relation.

When the study of pulse instability is extended to the QG six-layer model in Chapter 3, we have found that the growth rates of individual peaks can vary from $30 \%$ to $220 \%$ of that of the peak of the wave envelope. Therefore the waves in the leading edge of a wave packet may grow several times faster than the waves in the trailing edge in the exponential sense. This can not be predicted by temporal or spatial instability theories.

Direct comparison between temporal and pulse theory in Chapter 2 shows that pulse instability allows unstable waves to have wavenumbers beyond the short 
wave cut-off of the temporal theory. This is possible since we are comparing the real part of the local wavenumber in pulse theory with a global wavenumber in temporal theory. The local wavenumber in pulse theory contains a nonzero imaginary part, so that even if its real part is the same as the wavenumber in temporal theory, its frequency $\omega\left(k_{r}+i k_{i}\right)$ has been extended to the complex $k$ plane and is naturally different from that in temporal theory $\left(\omega\left(k_{r}\right)\right)$.

When a single pulse evolves as pulse instability predicts, the amplitude of a specific wavenumber in the energy spectrum still grows according to the growth rate of temporal instability. Therefore when pulse instability occurs, the most energetic wave is the most temporally unstable wave.

\section{Effect of Topography}

In Chapter 3, from the analysis of potential density and the spread ratio, we have shown that a minimum of three layers is necessary to address the effect of topography properly in the context of the Gulf Stream. The main reason is that the effect of topography on potential vorticity distribution is confined in a layer near the bottom and should be represented separately from the interior of the flow system. Comparison among two to six-layer models support this point. The comparison also shows that short waves are more sensitive to vertical resolution. In the case of pulse instability, a minimum of five layers is necessary to capture the characteristics of the instability. This is because the growth rates of individual peaks depend on the difference between the phase speed and group velocity. The latter depends on the derivative of the former and is more sensitive to vertical and horizontal resolution, especially in regions where the mean velocity changes rapidly.

\section{Effect of Ageostrophy}


In Chapter 4, we have found that the QG six-layer model overestimates substantially the maximum growth rate compared to the SW six-layer model (e.g. $40 \%$ higher in QG6 than that in SW6). This is consistent with the previous study by Orlansky (1969) that finite Rossby number reduces the growth rates of unstable waves. However, QG and SW models give comparable wavelengths and phase speeds of the main unstable modes (e.g. $349 \mathrm{~km}$ and $7.9 \mathrm{~km} /$ day in QG6 vs $363 \mathrm{~km}$ and $7.5 \mathrm{~km} /$ day in SW6). The growth rates of individual peaks in the two models agree very well as functions of the group velocity, though the maximum growth rates are still significantly different. The conclusion from this comparison is that the QG model gives results quantitatively different from those from the SW model, but there is no qualitative difference between the two models in terms of the first most unstable modes.

\section{Comparisons with Observations}

In Chapter 4, I have drawn connection between our model results and observations. In the comparison with observations, I focused on the studies by Lee and Cornillon (1996a,b) since only their work distinguishes the fastest growing wave from most energetic wave in a well-defined way, to the best of my knowledge. Despite some discrepancies among the models and the results of their analysis, it appears to be a robust common feature that the fastest growing wave has higher growth rate, wavenumber and frequency than the most unstable wave. And the differences between the most unstable and the fastest growing waves in the pulse instability models seem able to explain substantial parts of the differences observed. 


\subsection{Numerical Experiments}

\section{Development of a Single Pulse}

In the context of the Gulf Stream, it takes about 45 days for a single pulse disturbance to develop close to its asymptotic form. In that period the wave packet has moved downstream $1100 \mathrm{~km}$ to about $65^{\circ} \mathrm{W}$. This is still in the range where the Gulf Stream system has been intensively observed. Hence there appears to be enough space for the pulse instability to occur in the Gulf Stream. On 61st day, the fastest growing wave has a growth rate of $0.086 /$ day, which is $60 \%$ higher than the maximum envelope growth $0.053 /$ day. The former has a wavelength, phase speed and period of (310km, 14km/day, 22 days), and the latter (336km, 12km/day, 29 days).

It appears that the QG two-layer analytical and numerical model have comparable ratios of quantities between the fastest growing waves and most energetic waves despite the fact that the maximum growth rate in the numerical model is $18 \%$ lower than its analytical counterpart on 45 th day or $14 \%$ lower on 61 th day. This difference is mainly due to the $O(1 / t)$ contribution neglected in the analytical growth rate. When $t$ is as short as 45 days or 61 days, the main features of pulse instability already show up clearly in the numerical model. This suggests that the analytical results of SW6 and QG6 may also be carried over to their corresponding numerical models. And it seems reasonable to compare the analytical results of SW6 and QG6 directly with observations.

\section{Continuously Forced Problem}

In the continuously forced problem, we have found that the growth rates of individual peaks from the numerical runs scatter widely, especially in the region from $200 \mathrm{~km}$ to $1200 \mathrm{~km}$ downstream of the forcing location. It is clear that neither of 
the three types of instabilities can explain the pattern given the continuous forcing. However, the "observations" from the model runs have shown one trend predicted by pulse instability: short waves tend to move and grow faster than long waves. This can not be predicted from temporal or spatial instability. Unfortunately, due to a numerical difficulty, I have not been able to extend the analysis to the region further downstream, where the pulse instability has been found to work better from the single pulse experiment.

These numerical experiments again support the robust common features of pulse instability we have studied in Chapter 2 to 4 , that the fastest growing wave has higher growth rate, wavenumber and frequency than the most energetic wave. Because the differences between the most energetic and the fastest growing waves of pulse instability theory in the analytical models seem able to explain substantial parts of the differences observed and the single pulse experiment suggests the analytical results may be carried over to their corresponding numerical models, pulse instability theory appears to be the most appropriate model for the Gulf Stream.

In light of our findings from the continuously forced problem, it appears very difficult to extract analytical dispersion relation based on the numerical measurement, even in the simple numerical model which has many fewer complicating factors than the real Gulf Stream (e.g. no nonlinearity, no downstream variation of basic flow and topography, etc.). However, wavenumber bin-averaged rates tend to agree with pulse instability to some extent. Hence this experiment suggests that grouping observational data into bins of wavenumbers - the technique used by Lee and Cornillon (1996b) to find the fastest growing wave-is the most appropriate way to compare with analytical models.

The continuously forced problem does not support spatial instability. Hence it does not fit the way Johns (1988) and Kontoyiannis (1992) compared their model analytical dispersion relations with the observations. They extracted spatial growth 
rates from $\mathrm{EOF}$ analysis and compared them with equivalent or pseudo-equivalent spatial growth rates (ESGR or PESGR) from temporal instability discussed in Chapter 2 and 3 .

The greatest strength of this experiment is the use of time series of north wall displacement near the inlet as a forcing function, which is more realistic than the idealized forcing used in other models (e.g. Xue, 1991b, used a periodic forcing). In addition, we have tried to compare the results carefully with analytical theory.

\subsection{Limitation of This Study and Future Work}

Our research on pulse instability has provided a plausible explanation for the differences between the most energetic wave and the fastest growing wave observed in the Gulf Stream. However, we have not yet studied how the nonlinearity may alter the picture. In fact, Lee and Cornillon (1996b) attribute the observed differences to nonlinearity based on evidence that the fastest growing meanders $(350 \mathrm{~km})$ are somewhat shorter than the most energetic meanders $(427 \mathrm{~km})$. This observation is consistent with the theoretical notion (Pedlosky, 1981) that the most energetic wave realized at finite amplitude is longer than the linearly most unstable wave. However, we know from this thesis study that the observed differences are also consistent with linear pulse theory and continuously forced numerical experiments. It is conceivable that nonlinearity can also contribute to the difference, with the pulse instability mechanism setting up the main trend and the nonlinear effect gradually increasing its role and perhaps enhancing the trend as the ratios of wave amplitudes to their wavelengths increase.

From the single pulse experiment, we know that it takes about 45 days for a single pulse disturbance to develop close to its asymptotic form. However in 45 days, the maximum amplitude of wave packet is 17 times its initial amplitude 
$(\exp (.063 * 45)=17.0)$. Assuming an initial amplitude of $10 \mathrm{~km}$ at Cape Hatteras, the amplitude of the wave envelope will be $170 \mathrm{~km}$ at about $65^{\circ} \mathrm{W}$, about half of the wavelength. So the effect of nonlinearity may have already become significant, making it harder to compare data with analytical results directly. Without a study of nonlinear effects in the context of the Gulf Stream with realistic forcing, we cannot answer to what extent the nonlinearity will alter the results obtained from linear theories and linear numerical experiments. Neglecting nonlinearity is the greatest limitation of this study and I hope we will be able to address this issue in the future.

Swanson and Pierrehumbert (1993) carried out a nonlinear numerical study of pulse instability in a QG two-layer model. Their work was to study the fundamental nature of the midlatitude storm tracks in the atmosphere. Their experiments were similar to the single pulse experiments shown in Chapter 5. In their model, longer waves move faster and short waves appear in the upper stream end of the wave packet, in the linear stage of development. This pattern is consistent with analytical results of Farrell (1982) in the similar two-layer model with parameter tuned in atmosphere. And as I pointed out in Chapter 2 that this pattern is the opposite of the trend in the oceanic two-layer model.

As the nonlinearity became significant, they found that the short waves in the trailing edge started to equilibrate earlier at smaller amplitudes and phase speed started to decrease while fast moving and fast growing long waves continued to develop in the leading edge. This contrast eventually separated the wave packet into leading edge and trailing edge parts. This is unlikely to happen in the oceanic QG two-layer model since short waves travel faster and grow faster than long waves. If the effect of nonlinearity is also to slow down the phase speed and short waves start to equilibrate earlier than long waves, the trailing edge part will catch up the leading edge part so the development will be different from that observed by Swanson and Pierrehumbert. Thus, the results obtained from atmospheric studies are not directly 
applicable in the ocean and it is worth while studying nonlinear effect directly in the oceanic model.

The second limitation of this thesis study is that we have not considered any variation of velocity and topography along the stream. We can expect this variation may alter the picture of instability to some extent; for example, when the structure of the mean flow changes downstream, the growth rates of waves will adjust and this will alter the picture of pulse instability. When the flow moves down the stream, the underlying bottom topography becomes flatter and may cause the flow to be more unstable, and the wave patterns in the system may look different from our numerical experiments which assumes no variation of velocity and topography along the stream. Topography may also cause reflection of waves to form resonant global modes. Including any or all of these effects-nonlinearity, variations in jet structure, topography-may affect the picture of wave activities in the Gulf Stream to some extent. Despite the limitations of the linear model, however, the trend which shows up in the analytical and numerical models and in the observational analysis may still appear because the waves are still not periodic. However these limiting factors will make the comparison between data and models much more difficult.

The third limitation is that the model cannot run long enough so our results are based on the responses to segments of the forcing function but not the full, multiyear record. The fourth limitation is that, due to the limited model precision, the analysis in the continuously forced problem has not been extended to the further downstream region from $1000 \mathrm{~km}$ to $2000 \mathrm{~km}$ where the pulse instability has been found to work better from the single pulse experiments.

We know from Chapter 4 that in the SW six-layer model, the second most unstable mode has a maximum of growth rate very close to the most unstable mode (84\%) whereas in the QG six-layer model, this ratio is $41 \%$, and in the QG two-layer 
model $62 \%$. If we could run a SW six-layer numerical model, we might expect the results to have some significant differences from the QG two-layer model. 


\section{Appendices}

\section{A The Saddle Point Finder}

When a dispersion relation $\Delta(\omega, k)=0$ or $\omega=\omega(k)$ is defined numerically, there are two ways to locate a saddle point.

1. Direct search of $\omega_{s}$ for each $k_{r}$.

Plot real part of the frequency $\omega$ in complex $k$ plane. For any given $k_{r}$, we increase or decrease $k_{i}$ from real $k$ axis until hitting the point $\frac{\partial}{\partial k_{i}} \omega_{r}=0$ (see Figure A. 1). Repeat this procedure for adjacent value of $k_{r}$, and we will find a curve satisfying the saddle point condition $\frac{\partial}{\partial k_{i}} \omega_{r}=0$. For each point on the curve, group velocity $C_{g}=\frac{\partial}{\partial k_{r}} \omega_{r}=\frac{\partial}{\partial k_{i}} \omega_{i}$ can be calculated. So for each $k_{r}$ value, we will find a saddle point $\left(k_{s}, \omega_{s}, C_{g s}\right)$, where subscript 's' simply indicates a value at a saddle point. We then use $C_{g s}$ as an index to sort out $k_{s}$ and $\omega_{s}$ as functions of $C_{g s}$

This method is more direct but is less efficient numerically compared to the second method showed below. Moreover, it is more difficult to look for solutions in some segments of the curve such as the part between A and B when there are multiple values of $\omega$ corresponding to one value of $k_{r}$.

2. The saddle point finder.

This method can be easily illustrated in one dimensional search for a maximum or a minimum of a function $f(x)$ (see Figure A. 2). Both $f(x)$ and $x$ are real. To find the point with $\frac{\partial}{\partial x} f=0$, one can make an initial guess of $x_{i}$, and calculate $f_{1}=f\left(x_{i}-\Delta x\right), f_{2}=f\left(x_{i}\right)$ and $f_{3}=f\left(x_{i}+\Delta x\right)$. Then we can fit these three points with a second order polynomial $F(x)=a x^{2}+b x+c$. After $(a, b, c)$ are calculated, $\frac{\partial}{\partial x} F(x)=2 a x+b=0$ gives an estimate $x_{1}=\frac{-b}{2 a}$ for the next search. Drop one of 


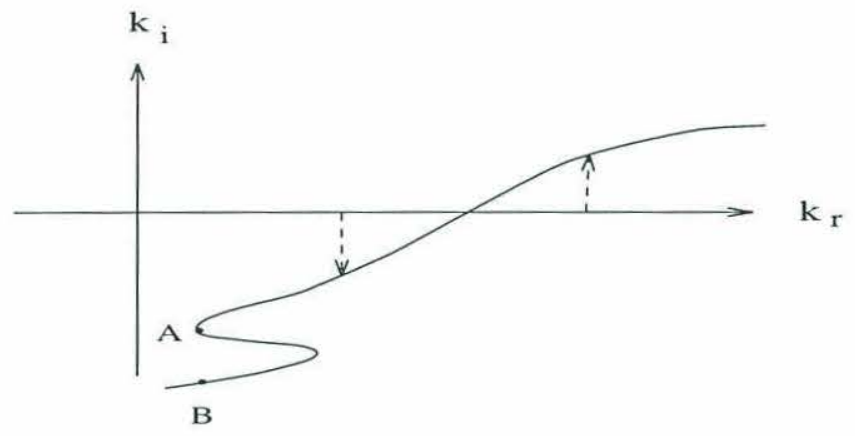

Figure A. 1: A sketch to show how to search for saddle points directly in complex $k$ plane.

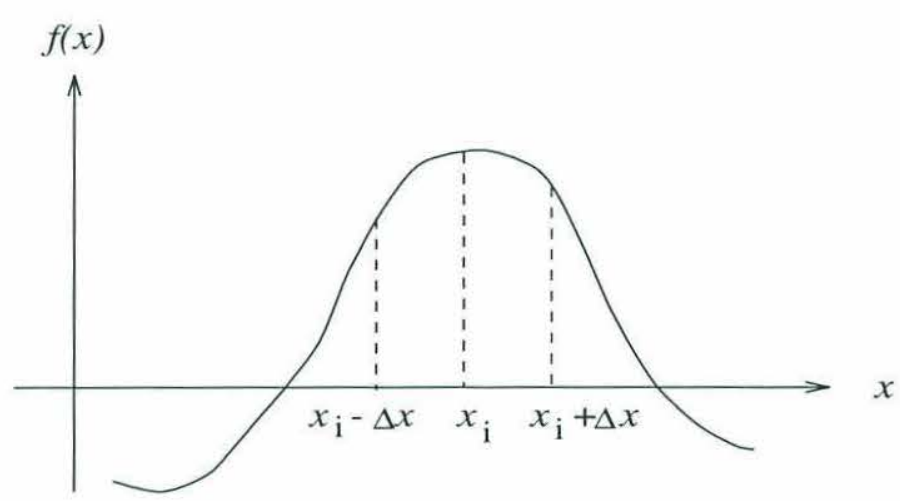

Figure A. 2: A sketch to show how to search for a maximum of a real function efficiently.

the three points $\left(x_{i}-\Delta x, x_{i} x_{i}+\Delta x\right)$ which is farthest away from $x_{1}$. With a new set of three points including $x_{1}$, the next prediction can be made. In such a way true location with $\frac{\partial}{\partial x} f=0$ can be found very efficiently.

Now we return to our problem of finding $\frac{\partial}{\partial k} \omega=0$. The above method can be applied directly except now $\omega$ and $k$ are complex. However, since $(\mathrm{a}, \mathrm{b}, \mathrm{c})$ can also be complex, we are still able to determine 6 parameters of $\left(a_{r}, a_{i}, b_{r}, b_{i}, c_{r}, c_{i}\right)$ from 6 given values $\left(\omega_{1 r}, \omega_{1 i}, \omega_{2 r}, \omega_{2 i}, \omega_{3 r}, \omega_{3 i}\right)$ and to find the location with $\frac{\partial}{\partial k} \omega=0$ similarly. This point with $\frac{\partial}{\partial k} \omega=0$ is automatically a saddle point if $\omega(k)$ is an analytical function of $k$ in a small region nearby.

The search for saddle points should start at the group velocity $C_{g}$ corresponding to the most unstable wave $\left(\omega_{\max }, k_{\max }\right)$ in temporal instability, since 
$\omega\left(k_{\max }\right)$ itself is a saddle point of function $\Omega=\omega-C_{g} k$. Then we increase or decrease $C_{g}$ to find a saddle point for $\left(C_{g}+\Delta C_{g}\right)$ or $\left(C_{g}-\Delta C_{g}\right)$. The result from each step is used as the initial guess to locate the next saddle point. The method was first used for this problem by Lin and Pierrehumbert (1993). The drawback of this approach is that it is very sensitive to initial guesses; hence the increment of $C_{g}$, $\Delta C_{g}$, must be very small, otherwise branch switching will happen. This sensitivity costs significant amount of computing time. I have modified the code by extrapolating the next saddle point from previous three saddle points (second order polynomial extrapolation) and reduced the computing time by an order of magnitude. 


\section{B Method for Solving Spatial Instability Prob- lem}

Similar to the temporal instability problem, we need to solve the dispersion relation $\Delta(\omega, k)=0$. However, as I mentioned in Sec. (2.3.2), an additional difficulty arises because the original linear eigenvalue problem for $\omega$ now become nonlinear problem for $k$. No standard software is available to solve the whole nonlinear problem as far as I know. I have used the following method to search for spatial instability corresponding to a few most temporally unstable branches of the dispersion equation.

The best place to start the search is the most unstable frequency $\omega_{\max }$ (complex) and wavenumber $k_{\max }$ (real) of a temporally unstable mode. For given $\omega=\omega_{\text {rmax }}$, use $k_{\max }-i \frac{\omega_{i \max }}{C_{\max }}$ as an initial guess. We contour $\Delta(\omega, k)$ on the $\left(k_{r}, k_{i}\right)$ plane around the initial guess to find the zero point of $\Delta(\omega, k)$. Because the initial guess may be quite far away from the solution, it may need several trial and error experiments to include the solution in the search domain. As long as the first point can be found, the rest is easier. After we have found the first solution $k_{0}$, which is typically complex, we decrease (or increase) $\omega$ by a very small amount $\Delta \omega_{1}$, take $k_{0}$ as the initial guess and search for the next solution $k_{1}$, then we can use $\left(k_{0}, k_{1}\right)$ to extrapolate $k_{2}$ linearly for $\omega_{2}=\omega_{\text {rmax }}-\Delta \omega_{1}-\Delta \omega_{2}$. After we have found three solutions, we use a second order polynomial to extrapolate the next initial guess. By using such interpolation, we are able to increase the step $\Delta \omega$ significantly without switching to other modes.

Briggs' method of selecting solutions of spatial instabilities described in Section 2.3.2 must be performed to confirm that solutions obtained correspond to amplifying waves. 


\section{Effects of Resolution and Channel Width}

This appendix is related to the QG analytical models used in Chapter 3. In this appendix, I will perform sensitivity tests on horizontal resolution and on the effect of channel width. I use a two-layer model with flat bottom and the modified profile described in Section 3.3. Figure C. 1 shows the differences between different resolutions used. The upper panel shows phase speeds vs real wavenumber $k$ and the lower panel temporal growth rate $\omega_{i}$ vs real wavenumber $\mathrm{k}$. Solid curves are associated with $\delta y=2.5 \mathrm{~km}$; dashed, $5 \mathrm{~km}$; and dotted, $10 \mathrm{~km}$. Channel width is fixed at $300 \mathrm{~km}$ for all three experiments. The figure only shows the three most unstable curves. As we can see from the figure, the differences among the results associated with all three resolutions are small and the results converge when the resolution increases. Hence I am confident that $\delta y=5 \mathrm{~km}$ or $10 \mathrm{~km}$ is adequate for all the other experiments.

Figure C. 2 shows the difference in phase speeds and temporal growth rates resulting from channel widths of $300 \mathrm{~km}$ and $400 \mathrm{~km}$. Solid curves are associated with channel width of $400 \mathrm{~km}$ and dashed $300 \mathrm{~km}$. Model resolution is fixed at $2.5 \mathrm{~km}$. The difference between the two cases is insignificant and hence I will use channel width of $300 \mathrm{~km}$ for all other experiments except where otherwise specified. 

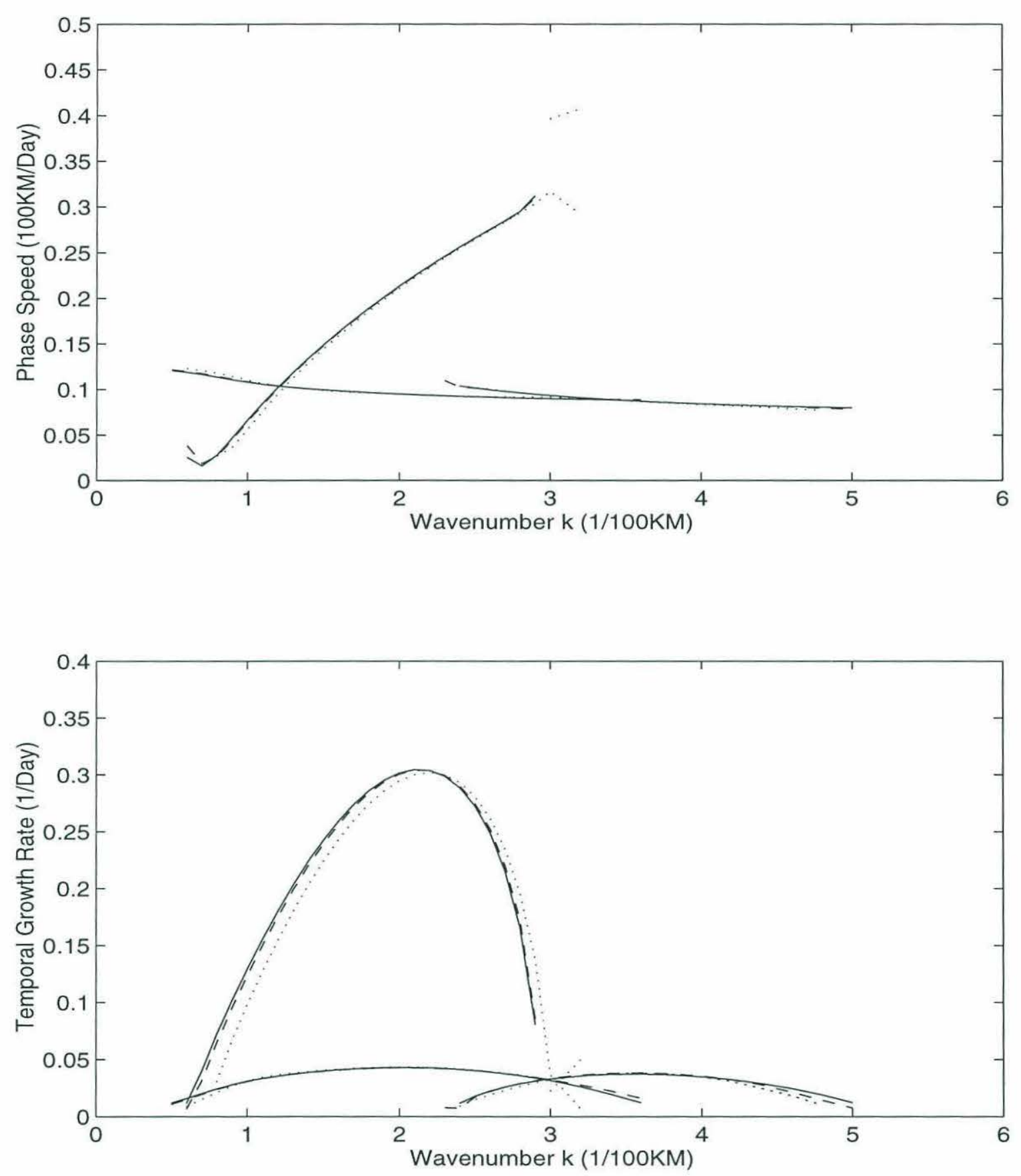

Figure C. 1: Resolution Test. Upper panel: Phase speeds vs real wavenumber $k$. Lower panel: Temporal growth rate $\omega_{i}$ vs real wavenumber $\mathrm{k}$. Solid curves for $\delta y=2.5 \mathrm{~km}$; dashed, $5 \mathrm{~km}$; and dotted, $10 \mathrm{~km}$. Channel width is fixed at $300 \mathrm{~km}$ 

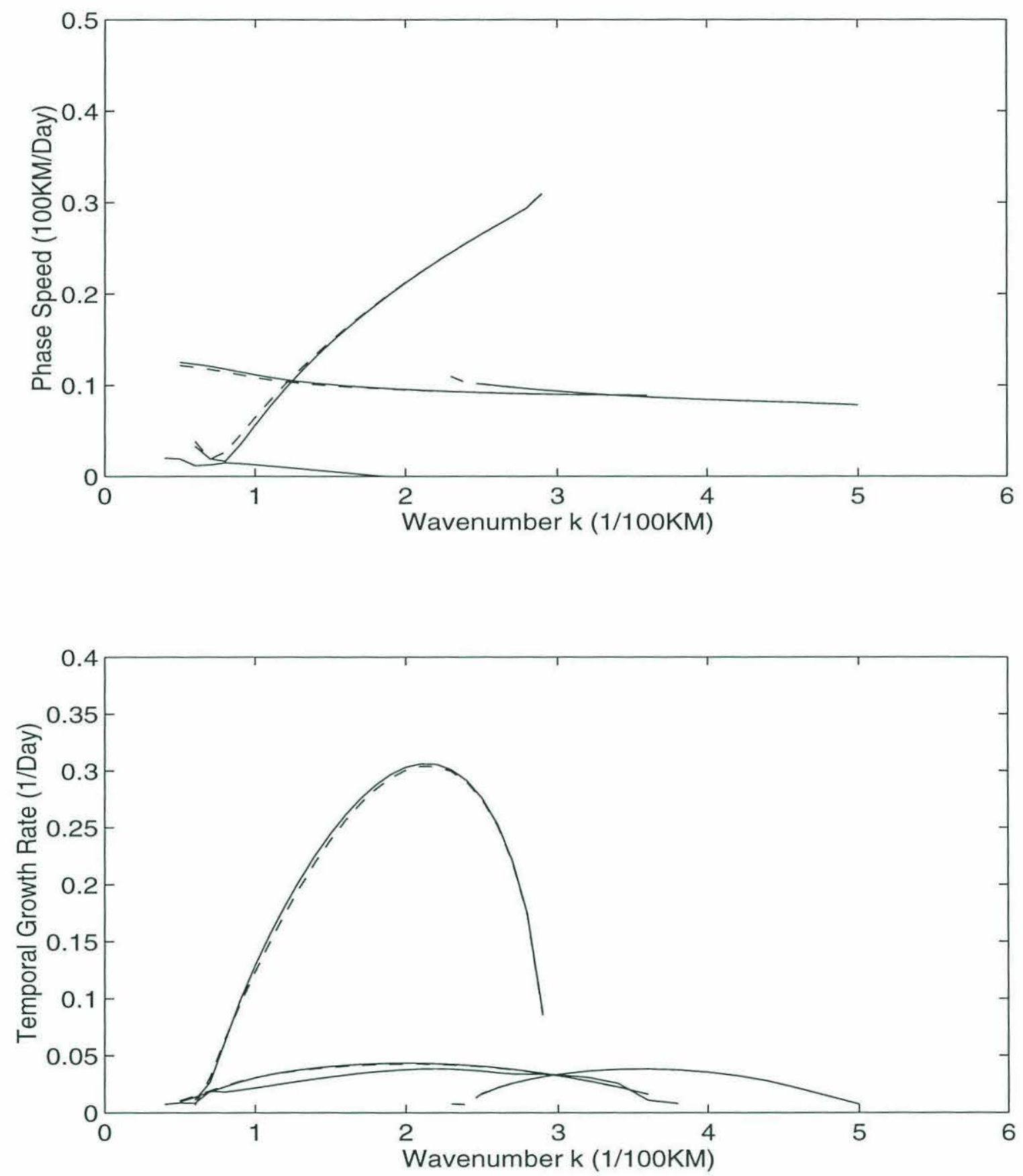

Figure C. 2: Effect of channel width. Upper panel: Phase speeds vs real wavenumber $k$. Lower panel: Temporal growth rate $\omega_{i}$ vs real wavenumber $\mathrm{k}$. Solid curves for channel width $=400 \mathrm{~km}$; dashed, $300 \mathrm{~km} . \delta y$ is fixed at $5 \mathrm{~km}$ 


\section{References}

Abramov, A. A., B. A. Tareyev, V. I. Ul'yanova, 1972a: Baroclinic instability in Kochin's two-layer model of a frontal wave on the beta plane. Izv. Atm. and Oc. Phys. 2,131-141.

Abramov, A. A., B. A. Tareyev, V. I. Ul'yanova, 1972b: Instability of a two-layer geostrophic current with antisymmetric velocity profile in the top layer. Izv. Atm. and Oc. Phys. 8,1017-1028

Barth, J. A., 1987: Stability of a coastal upwelling front over topography. PH. D. Thesis. MIT/WHOI, WHOI-87-84.

Bender, C.M. and S. Orszag, 1978: Advanced Mathematical Methods for Scientists and Engineers. McGraw-Hill Book Company.

Boudra, D. B., R. Bleck and F. Schott, 1988: A numerical model of instabilities in the Florida Current. J. Mar. Res., 46, 715-751.

Bower, A. S., 1989: Potential vorticity balances and horizontal divergences along particle trajectories in Gulf Stream meanders east of Cape Hatteras. J. Phys. Oceanog. 19, 1669-1681.

Bretherton,F.P., 1966: Baroclinic instability and the short wavelength cut-off in terms of potential vorticity. Quarterly Journal of the Royal Meteorological Society 92 .

Briggs, R.J., 1964: Electron stream interaction with plasmas. Chapter 2. MIT press, 8-46.

Cushman-Roisin, B., L. J. Pratt, and E. Ralph, 1993: A general theory of equivalent barotropic thin jet. Journal of Physical Oceanography, 23, 91-103. 
De Szoeke, R. A., 1975: Some effects of bottom topography on baroclinic stability. J.Mar. Res., 33, 93-122.

Farrell, B. F., 1982: Pulse asymptotics of the Charney baroclinic instability problem. J. Atmos. Sci., 39, 507-517

Gangopadhyay, A., 1990: Wind forcing of the Gulf Stream: A space-time analysis. Ph.D. thesis, Graduate School of Oceanography, University of Rhode Island, 190pp.

Gaster, M., 1962: A note on a relation between temporally increasing and spatially increasing disturbances in hydrostatic instability. Journal of Fluid Mechanics, 14, 222-224.

Griffiths, R. W., P. D. Killworth and M. E. Stern. 1981: Ageostrophic instability of ocean currents. J. Fluid Mech., 117, 343-377.

Hall, M. M. and Fofonoff, N.P., 1993: Down stream variation of the Gulf Stream from $68^{\circ} \mathrm{W}$ to $55^{\circ} \mathrm{W}$. J. Phys. Oceanog. 23, 225-249.

Halliwell, G. R. Jr. and C. N. K. Mooers, 1983: Meanders of the Gulf Stream downstream from Cape Hatteras. Journal of Physical Oceanography, 13, 12751292.

Halkin, D. and T. Rossby, 1985: The structure and transport of the Gulf Stream at $73^{\circ}$ W. J. Phys. Oceanog. 15, 1439-1452.

Hart, J. E., 1974: On the mixed stability problem for quasi-geostrophic ocean currents. J. Phys. Oceanog. 4, 349-356

Hogg, N. G., 1976: On spatially growing baroclinic waves in the ocean. J. Fluid Mech., 78, 217-235. 
Ikeda, M., 1983: Linear instability of a current flowing along a bottom slope using a three-layer model. J. Phys. Oceanog. 13, 208-223

Johns, W. E., 1988: One-dimensional baroclinically unstable waves on the Gulf Stream potential vorticity gradient near Cape Hatteras. Dyn. Atmos. Oceans, 11, 323-350.

Killworth, P. D. and M. E. Stern, 1982: Instabilities on density-driven boundary currents and fronts. Geophys. Astrophys. Fluid Dyn., 23, 1-28.

Killworth, P.D., 1983: Long-wave instability of an isolated front. Geophys. Astrophys. Fluid Dyn., 24, 235-258.

Killworth, P. D., N. Paldor and M. E. Stern, 1984: Wave propagation and growth on a surface front in a two-layer geostrophic current. Journal of Marine Research, 42, 761-785.

Kontoyiannis, H., 1992: Variability of the Gulf Stream path between $74^{\circ} \mathrm{W}$ and $70^{\circ} \mathrm{W}$ : observations and quasi-geostrophic modelling of mixed instabilities. $\mathrm{Ph}$. D. dissertation, Graduate School of Oceanography, University of Rhode Island, $137 \mathrm{pp}$.

Leaman, K. D., E. Johns and T. Rossby, 1989: The average distribution of volume transport and potential vorticity with temperature at three sections across the Gulf Stream. J. Phys. Oceanogr.,19, 36-51.

Lee, S. and G. T. Csanady, 1994: Instability waves in the Gulf Stream front and its thermocline layer. J.Mar. Res., 52, 837-863.

Lee, T., 1993: Variability of the Gulf Stream path from $75^{\circ}$ to $60^{\circ} \mathrm{W}$ observed from satellite infrared imagery. Spring AGU meeting, Baltimore, MD, May, 1993.

Lee,T., P. Cornillon, 1996a: Propagation of Gulf Stream meanders between $74^{\circ}$ and $70^{\circ}$ W. J. Phys. Oceanogr., 26, 205-224. 
Lee,T., P. Cornillon, 1996b: Propagation of Gulf Stream meanders between $75^{\circ}$ and $45^{\circ}$ W. J. Phys. Oceanogr., 26, 225-241.

Lin, S.J., R.T. Pierrehumbert, 1993. Is the midlatitude zonal flow absolutely unstable? J. Atmos. Sci.,50, 505-517

Lipps, F. B., 1963: Stability of jets in a divergent barotropic fluid. J. Atmos. Sci.,20, $120-129$

Michalke, A., 1965: On spatially growing disturbances in an invisid shear layer. Journal of Fluid Mechanics,23, 521-544

Nikitin, O. P. and B. A. Tareyev, 1972: Meanders of the Gulf Stream interpreted as resultants of baroclinic instability predicted by a simple two-layer model. Izv. Atm. and Oc. Phys. 8, 973-980

Luther, M.E. and J. M. Bane, Jr., 1985: Mixed instabilities in the Gulf Stream over the continental slope. J. Phys. Oceangr., 15, 3-23.

Orlanski, 1969: The influence of bottom topography on the stability of jets in a baroclinic fluid. J. Atmos. Sci., 26, 1216-1232.

Orlanski and M. D. Cox, 1973: Baroclinic instability in ocean currents. Geophys. Fluid Dyn., 4, 297-332.

Paldor, N., 1983a: Stability and stable modes of coastal fronts. Geophys. Astrophys. Fluid Dyn., 27, 217-229.

Paldor, N., 1983b: Linear stability and stable modes of geostrophic fronts. Geophys. Astrophys. Fluid Dyn., 24, 299-326.

Paldor, N., 1991: Shortwave stabilities of coastal currents. Geophys. Astrophys. Fluid Dyn., 58, 225-241. 
Pedlosky, J. 1964a: The Stability of Currents in the Atmosphere and the Ocean: Part I . JAS,21,201-218

Pedlosky, J. 1964b: The Stability of Currents in the Atmosphere and the Ocean: Part I . JAS,21,342-353.

Pedlosky, J. 1981: The nonlinear dynamics of baroclinic wave ensembles. Journal of Fluid Mechanics,102, 169-209

Pedlosky, J. 1986: Geophysical Fluid Dynamics. Second edition. Springer-Verlag, NY.

Pierrehumbert, R. T., 1986: Spatially amplifying modes of the Charney baroclinicinstablity problem. Journal of Fluid Mechanics,170, 293-317.

Phillips, N. A., 1964: An overlooked aspect of the baroclinic instability problem. Tellus, 16, 268-270.

Sela J. and S. J. Jacobs, 1971: Ageostrophic effects on Gulf Stream instability. J. Atmos. Sci.,28, 962-967

Simmons, A.J., and B.J. Hoskins, 1979: The Downstream and upstream development of unstable baroclinic waves. J. Atmos. Sci.,36, 1239-1254

Stone, P., 1978: Baroclinic Adjustment. J. Atmos. Sci.,35, 561-571

Talley, L. D., 1983a: Radiating barotropic instability. J. Phys. Oceanog. 13, 972-987

Talley, L. D., 1983b: Radiating instabilities of thin baroclinic jets. J. Phys. Oceanog. $13,2161-2180$

Tareyev, B. A., 1965: Unstable Rossby waves and the instability of ocean currents. Izv. Atm. and Oc. Phys. 1,426-438 
Tracey, K. L., and D. R. Watts, 1986: On Gulf Stream characteristics near Cape Hatteras. J. Geophys. Res., 91, 7587-7602.

Watts, D. R., 1983: Gulf Stream variability. In: Eddies in Marine Science, edited by A. Robinson, pp. 114-144, Springer-Verlag, New York.

Watts, D. R., and W. E. Johns, 1982: Gulf Stream meanders: observations on propagation and growth. Journal of Geophysical Research, 87, 9467-9476.

Xue, H., 1991a: Stability Analyses of the Gulf Stream Front Using the linearized Primitive Equations. Ph. D. Thesis, part I.

Xue, H., 1991b: Numerical Simulation of the Gulf Stream Meanders Using a Three Dimensional Primitive Equation Model. Ph. D. Thesis, part II. Princeton Univ.

Xue, H., and G. Mellor, 1993: Instability of the Gulf Stream front in the South Atlantic Bight, J. Phys. Oceanogr., 23, 2326-2350. 